\title{
Who Was Who in the Aether of the Weimar Republic? Tracing the Callbooks That Never Existed
}

\section{Introduction}

James Clark Maxwell predicted electro-magnetic waves theoretically in the 1860ies and Heinrich Hertz confirmed their existence by his experiments in 1887. At the same time, attempts began by Ferdinand Braun, Schneider (EK4ABM), Marconi and others to use and commercialize radio waves for wireless communication. Around 1905, the discovery sparked the interest of radio enthusiasts, mostly young people, who started to experiment with simple receivers and sometimes powerful transmitters for contacting each other [Wiegand, 1908]. This activity was referred to as Amateur Radio (or, for obscure reasons, Ham Radio), to distinguish it from commercial two-way radio services of the military, on ships, etc, which slowly began to emerge around 1912. Although only a small fraction of the population patricated in amateur radio, it was essential in the development of radio technology and in the education of engineers and scientists.

The biggest societal influence of amateur radio was the invention of broadcast, which soon became independent. After WW1, governments started to install and run big transmitters to broadcast scheduled content, like news and entertainment. In Germany, broadcast was promoted by Hans Bredow (1879-1959, Founding director of the Department for Wireless Telegraphy in the Reichspostministerium since 1919, ousted by the NS government in February 1933) and Eugen Nesper (1879-1961, Founder of the Deutscher Radio-Klub e. V. in 1923), leading 1923 to the first broadcast station in Berlin. Broadcast, accessible with modest commercially produced radio receivers, quickly attracted millions.

After WW1, radio amateurs were restricted by international treaties to wavelengths shorter than $200 \mathrm{~m}$, which were considered useless by commercial and governmental services. The restriction turned out to be advantageous, as radio amateurs discovered 1921/1922 that these short wavelengths enabled worldwide communication with moderate antenna size and transmitting power [De Soto, 1936]. The discovery made amateur radio a worldwide community that attracted people across different layers of society and enabled their education. Following the example of the American Radio Relay League in the United States, radio amateurs in Europe started around 1923 to broadly organize radio clubs and publish amateur radio journals. Maybe the first instance of a maker movement, these activities broadly established a technical culture, which not only enabled participation in amateur radio, but also helped create the vibrant industrial and scientific development during the 1920ies and 30ies in many countries [Haring, 2006].

In most countries, Amateur Radio was legally permitted to citizens that underwent an exam, proving their technical and operational abilities, to obtain an amateur radio license. Not so in Germany. The period from 1923 to 1933 during the Weimar Republic has been referred to as the "romantic period" of Amateur Radio in Germany. It was also considered a dire period, because, under the Prussian Telegraph Law from 1892, amateur radio was simply "verboten". The only legal activity was reception of radio signals, which still required a permit, the "Audionversuchserlaubnis" [K]. The name of the largest German amateur radio club, Deutscher Amateur Sende Dienst (DASD), had transmission in its name, but for most members this was just a dream. Starting in 1925, there were a few exceptions, official transmission licenses with callsigns KA0-KZ3 were issued to some clubs, companies, and educational institutions (Pre-1927 official "lis" calls). These official callsigns were converted in 1927 to EK4xxx, in 1929 to D4xxx. But most private citizens who wanted to actively participate in amateur radio had to work around the law. The DASD designed in 1927 a system for unofficial callsigns, of the form EK4xx, in which the first letter of the suffix encoded region. In 1929 these calls were converted to D4xx callsigns. To blend in with official callsigns the DASD designed in 1930 a system to convert D4xx calls into unofficial D4xxx callsigns. The unofficial stations sent out anonymous QSL cards (see some examples below) and could not be found in amateur radio callbooks.

Because "unlis" operations had to be clandestine, the operators behind most unofficial call signs have been a mystery. This is the first comprehensive attempt to identify and compile "unlis" holders of German call signs used in the period from 1923 to 1933, in some cases to later. Beginning with 34 "lis" licenses on May 15, 1933 [CQ-MB 8/1933], the Nazi regime started to issue official "lis" licenses. We hope that this listing can shed some light on a chapter of amateur radio, which for many reasons, has remained in the shadow. We added here and there a bit of biographical information, which allows some climses how amateur radio during the Weimar Republic influenced history and, in turn, was affected by history:

1) Some operators later became scientists or leading technology developers, for example: EK4AAL, EK4AAR, EK4ABM, EK4ACI, EK4AV, EK4CM, EK4GP, EK4LV, EK4VF, EK4XU

2) Some were involved in intelligence activities: EK4AB: Abwehr, later BND - Organisation Gehlen; EK4KQF: BND; EK4KU: BND; EK4TH: Abwehr agent in Mexico; EK4TY: Abwehr agent in Argentina; EK4XF: hired by CIA, then inactive; D4OMO: hired by CIA; EK4HH(2): MfS Referatsleiter in the GDR.

3) Some became victims/refugees of the Nazi dictatorship that ended the Weimar Republic: Kc2, EK4ABK(2), EK4ACZ, EK4AG, EK4AH(1), EK4CL, EK4CM, EK4JB, EK4QV(1), EK4QY(1), EK4SAR(1), EK4YAE, D4FLA, D4ZGM, UN7DD

4) Holders of about $6 \%$ of active EK calls remain unknown: Wanted: EK4AX, DA, DC, DD, DN, DV, FG, GQ, HA, HF, HP, II, MC, MQ, NB, NI, NU, NW, NX, NZ, OJ, OR, OS, OY, QD, QM, QN, QO, RL, RP, RZ, SL, TG, UT, VO, VW, XA, XC, XG, XL, XW, $\mathrm{XX}, \mathrm{YA}, \mathrm{YT}, \mathrm{YW}, \mathrm{YZ}, \mathrm{ZA}, \mathrm{ZZ}$

In spite of the gaps and inaccuracies in this compilation, we hope that it is still helpful information for those interested in a quite unexplored chapter of amateur radio history. Maybe this can even inspire some to continue the investigations. Please help to make this list better, send corrections and additional information with sources to k6ee@arrl.net. 


\section{Einführung}

James Clark Maxwell sagte um 1860 die Möglichkeit elektromagnetischen Wellen voraus und Heinrich Hertz bestätigte 1887 die Existenz solcher Wellen durch seine Laborexperimente. Fast gleichzeitig begannen Versuche von Ferdinand Braun, Schneider (EK4ABM), Marconi und anderen, Radiowellen für Kommunikation zu nutzen und zu kommerzialisieren. Radiowellen übten eine magische Faszination auf eine breite Bevölkerungsschicht aus. Vor allem junge Leute begannen bald darauf, in USA schon um 1905, mit einfachen Empfängern und teils leistungsstarken Sendern zu experimentieren und miteinander in Kontakt zu treten [Wiegand, 1908]. Diese Aktivität wurde als Amateurfunk (Amateur Radio) bezeichnet, um von kommerziellen Funkdiensten in Militär und auf Schiffen zu unterscheiden, die in USA etwa um 1912 ihre Arbeit begannen. Obwohl nur ein kleiner Anteil der Bevölkerung am Amateurfunk partizipierte, spielte dieser dennoch eine wichtige Rolle bei der Entwicklung von Radiotechnik und für die Ausbildung von Ingenieuren und Wissenschaftlern.

Der wohl größte gesellschaftliche Beitrag des Amateurfunks war die Erfindung des Rundfunks, der sich bald verselbständigte. Nach dem ersten Weltkrieg begannen Regierungen grosse Sender zu installieren und betreiben, die nach einem Sendeplan Nachrichten und Unterhaltung boten. In Deutschland wurde der Rundfunk von Hans Bredow (1879-1959, Gründungsdirektor der Abteilung für drahtlose Telegraphie im Reichspostministerium seit 1919, 1933 abgesetzt vom NS Regime) und Eugen Nesper (1879-1961, Gründer des Deutschen Radio-Klub e. V., 1923) eingeführt. Die erste deutsche Rundfunkstation ging 1923 in Berlin in Betrieb. Rundfunk, der mit kleinen, bald kommerziell gefertigten Empfängern zu hören war, zog in kurzer Zeit ein Millionenpublikum an.

Nach dem ersten Weltkrieg wurde den Funkamateuren in internationalen Verträgen lediglich Bänder mit Wellenlängen unter 200 Meter zugeteilt, welche als nutzlos angesehen wurden - kommerzielle Funkdienste und Rundfunk durften längere Wellen benutzen. Die Amateure machten aus der Not eine Tugend, sie entdeckten 1921/22, dass auf den ihnen zugeteilten Frequenzen weltumspannende Verbindungen mit relativ kleinen Sendern und Antennen möglich sind [De Soto, 1936]. Amateurfunk wurde nun eine weltumspannende Gemeinschaft und zog Bürger aus unterschiedlichsten Gesellschaftsschichten an. Dem Beispiel des amerikanischen Vereins "American Radio Relay League" folgend, wurden in Europa um 1923 zahlreiche Radiovereine und Funkzeitschriften gegründet. Diese Aktivitäten brachten technisches Wissen in der Bevölkerung, welches für die Teilnahme am Amateurfunk unabdingbar war und darüber hinaus eine technische Kultur etablierte, welche die rasante Entwicklung der Industrieländer in den 20iger und 30iger Jahren mit ermöglichte [Haring, 2006].

In den meisten Ländern war es per Gesetz erlaubt, nach dem Absolvieren einer Prüfung in Funktechnik, Gesetzen und Telegraphie, eine Amateurfunkstation zu betreiben. Nach bestandener Prüfung erhielt der Kandidat von den Behörden ein Rufzeichen, eine Kennung, die im Funkverkehr zu benutzen war. Nicht so in Deutschland. Die Zeit von 1923-1933 während der Weimarer Republik wird manchmal als die romantische Periode des Amateurfunks in Deutschland bezeichnet. Zutreffender ist es wohl, diese Periode elend zu nennen, denn unter dem Preussischen Telegraphiegesetz von 1892, das immer noch galt, war Amateurfunk schlichtweg verboten. Die einzige legale Aktivität war der Empfang, und sogar dieser war staatlich reglementiert, es war eine Genehmigung, die Audionversuchserlaubnis erforderlich [K]. Daher blieb das "Senden" im Namen des größten Amateurfunkklubs, des Deutscher Amateur Sende Dienst (DASD) für die meisten Mitglieder nur ein Traum. Um 1925 gab es einige Ausnahmen, es wurden einige wenige Sendelizenzen and Klubs, Betriebe und einige Universitätsinstitute vergeben. Die ursprünglichen Rufzeichen Ka0-Kz9 wurden 1927 umgewandelt zu EK4xxx, und in 1929 zu D4xxx. Aber der Großteil von Privatleuten war entweder auf das legale Hören beschränkt oder musste das Gesetz umgehen. Letzteres wurde mit deutscher Gründlichkeit getan, der DASD entwarf 1927 ein System für inoffizielle "unlis" Rufzeichen, vom Format EK4xx, in dem der erste Buchstaben im Suffix die Region kodierte. Der Prefix EK wurde 1929 durch D ersetzt: D4xx. Schließlich wurde 1930 vom DASD eine Regel angewandt um die als illegal erkennbaren D4xx Rufzeichen in D4xxx Rufzeichen umzuwandeln, welche von legalen schwerer unterscheidbar waren. Die Inoffiziellen versandten anonyme QSL Karten (siehe nächste Seite) and waren nicht in den Rufzeichenlisten enthalten.

$\mathrm{Da}$ "unlis" Operationen aus Sicherheitsgründen anonym bleiben mussten, ist die Identität vieler dieser Stationen bis heute mysteriös und ungeklärt. Dies ist der erste Versuch, deutsche "unlis" Amateurfunkstationen in der Periode 1923 bis 1933, und in einigen Fällen später, vollständig zu dokumentieren. Am 15. Mai 1933, an zunächst 34 vormals Unlizensierte [CQ-MB 8/1933], begann das Nazi regime "lis" Liebhaberfunksendergenehmigungen zu vergeben. An einigen Stellen sind biographische Informationen hinzugefügt, welche Einblicke in Wechselwirkungen zwischen dem Amateurfunk der Weimarer Republik und dem allgemeinen Geschichtsverlauf ermöglichen:

1) Einige, der Funkamateure wurden später Wissenschaftler und Entwickler von Schlüsseltechnologien, zum Beispiel: EK4AAL, EK4AAR, EK4ABM, EK4ACI, EK4ACZ, EK4AV, EK4CM, EK4GP, EK4VF, EK4XU

2) Einige waren in Geheimdiensttätigkeiten involviert: EK4AB: Abwehr, später BND - Organisation Gehlen; EK4KQF: BND; EK4KU: BND; EK4TH: Abwehr Agent in Mexico; EK4TY: Abwehr Agent in Argentinien; EK4XF: angeheuert vom CIA, dann jedoch inaktiv; D4OMO: angeheuert vom CIA; EK4HH(2): MfS Referatsleiter in the DDR.

3) Einige der Pioniere des Amateurfunks in Deutschland wurden Verfolgte des Nazi Regimes: Kc2, EK4ABK(2), EK4ACZ, EK4AG, EK4AH(1), EK4CL, EK4CM, EK4JB, EK4QV(1), EK4QY(1), EK4SAR(1), EK4YAE, D4FLA, D4ZGM, UN7DD

4) Ungefähr 6\% der aktiven EK calls sind nach wie vor unidentifiziert: Gesucht: EK4AX, DA, DC, DN, DV, FG, GQ, HA, HF, HP, II, MC, MQ, NB, NI, NU, NW, NX, NZ, OJ, OR, OS, OY, QD, QM, QN, QO, RL, RP, RZ, SL, TG, UT, VO, VW, XA, XC, XG, XL, XW, XX, YA, YT, YW, YZ, ZA, ZZ

Trotz verbleibender Lücken und Ungenauigkeiten, hoffen wir, dass die vorliegende Liste nach beinahe hundert Jahren etwas Licht in ein wenig beleuchtetes Kapitel deutscher Amateurfunkgeschichte bringen kann - und vielleicht einige zu weiteren Nachforschungen anregt. Bitte senden Sie eventuelle Korrekturen und Ergänzungen an k6ee@arrl.net. 


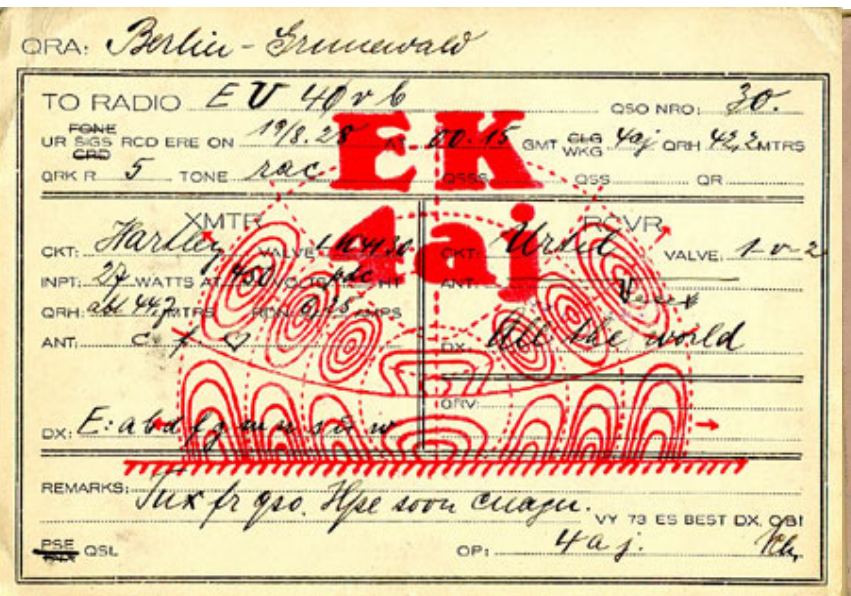

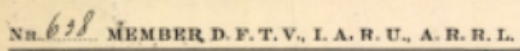
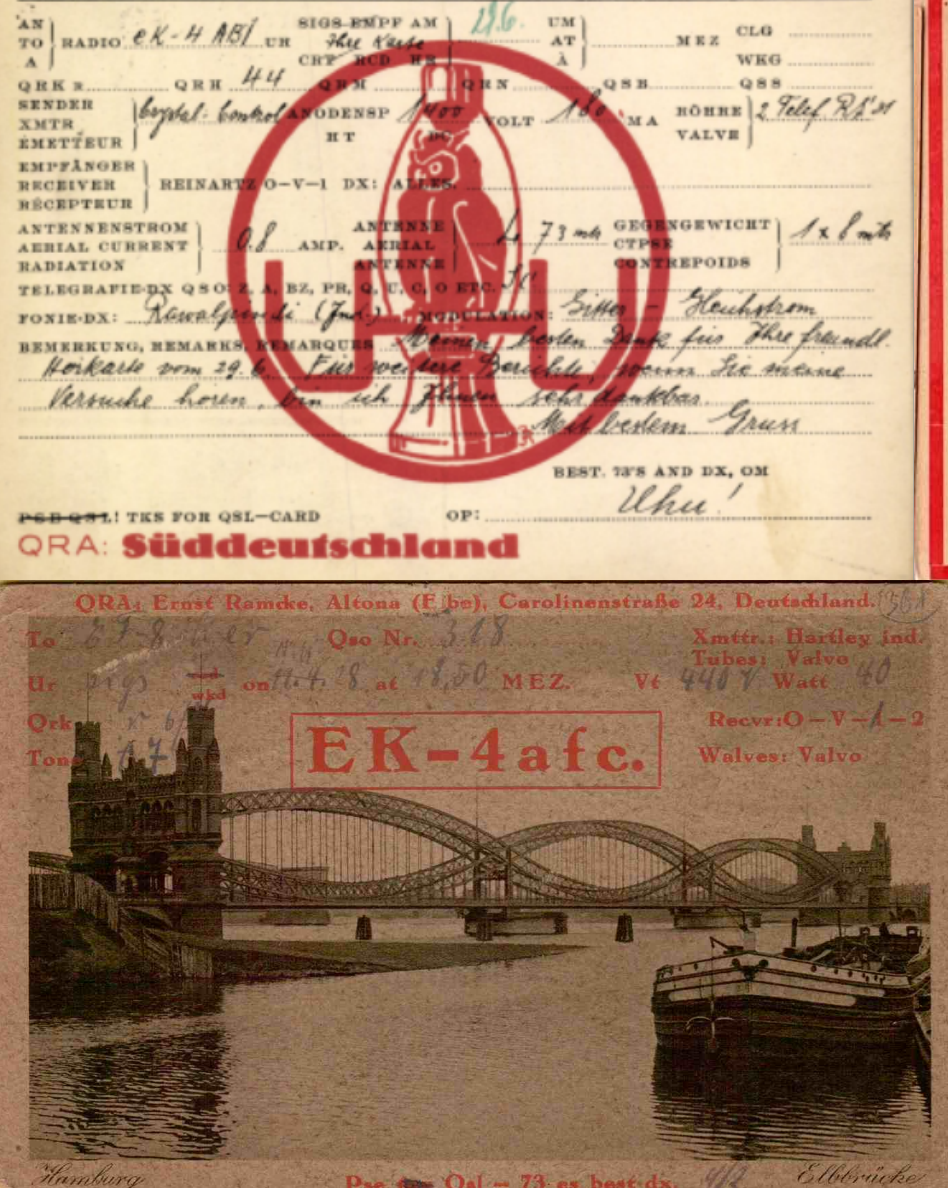

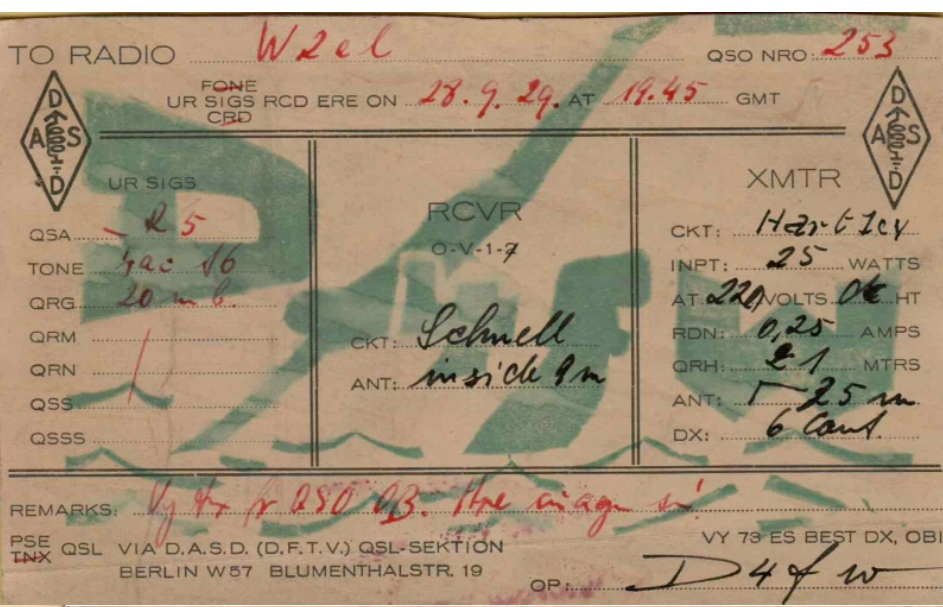

To Radio el 8 do $\hat{M}$ ur sigs wkd hr 23. 9. 28. at 2050 GMT.



QRA: Deutschland-Stuttgart. Qso Nr.

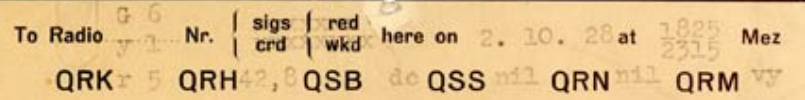

Transmitter



Dx: Europa, Afrika, Nord-Sued-Amerika, Asien, Neuseeland, Australien.


To Radio: EF $8 \mathrm{Ch} E R$

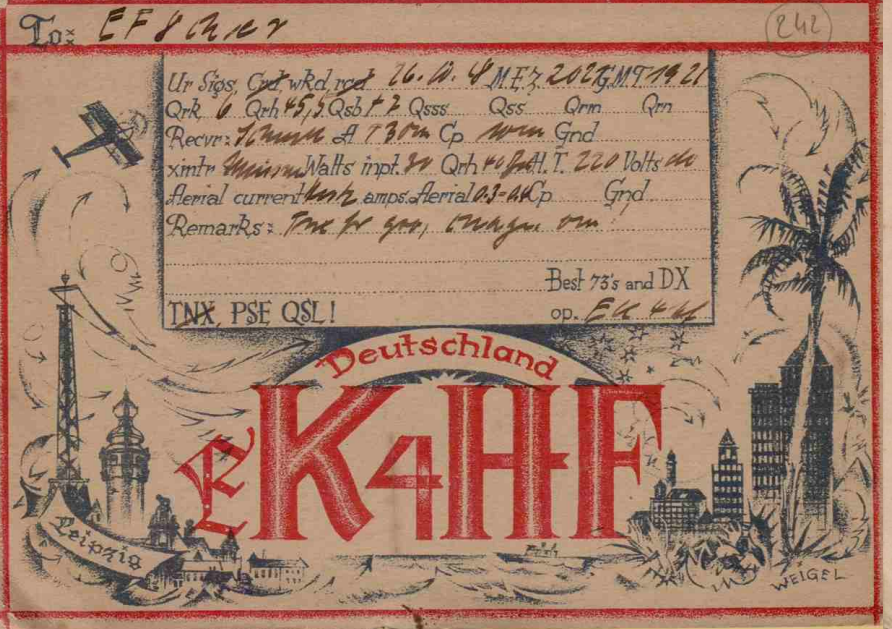


Systems of official and unofficial calls of amateur radio stations during the Weimar Republic in Germany

Official calls: Starting 1925, some exceptions were made, and permits issued to a few radio clubs, companies and educational institutions: $\mathrm{Ka} 0-\mathrm{Kz} 9 \quad$ legale Versuchsstationen (ab 1925)

On 1.2.1927 the system of official call signs was changed to:

EK4AAA-EK4AEY legale Versuchsstationen (ab 1.2.1927)

D4AAA-D4AEY legale Versuchsstationen (ab 1.1.1929)

D4DAA-..

Heer (ab 1928)

D4KAA-..

D4LAA-..

Kavallerie (ab 1928)

Marine (ab 1928)

Unofficial calls: In addition to the official "lis" stations, "unlis" stations appeared on the bands as well. First they used fantasy calls, like UHU, BABY, MARS. As early as Oct. 1925 the first unlis calls of the format K4xx were used [J:3], for example K4LD. In 1927 unofficial (E)K4xx calls were coordinated by the German amateur radio club DASD:

EK(D)4AA-EK(D)4ZZ "unlis"

The first letter in a two letter suffix encoded geographic region [J:3]: A, C, W: Berlin; B: Brandenburg und Pommern; D: Provinz Sachsen; E: Ostpreussen; F, T: Nordmark - Hamburg, Lübeck und Schleswig-Holstein; G: Schlesien; H, M: Sachsen und Vogtland; I: Thüringen; J: Osnabrück; K: Nordhessen; L, R: Rheinland und Westfalen; N: Südhessen; O: Baden; P: Bremen; Q: Braunschweig und Hannover; (S: Saarland;) U: Bayern; V: Franken; X: Württemberg; (Y: ?) Z: Freie Stadt Danzig, nicht im Deutschen Reich.

Some calls still were "freely invented", without adhering to the DASD coordination scheme: EK4AL, Stuttgart, EK4HL, Hamburg.

On 1/9/1930 the DASD transitioned "unlis" calls to three letter suffixes because it was too easy to tell "unlis" and "lis" stations apart [J:8]: DASD Transformation key: EK4AZ -> D4XZA (with X a random letter, sometimes changed every month).

Prefix changes: The prefix for Germany changed on 1/3/1926 from K to EK (e.g., Ky8 became EKy8), and in 1929 from "EK" to "D" (e.g., EK4ABC became D4ABC, EK4CM became D4CM). Because suffixes remained, the prefix is unimportant for identifying call holders.

DE numbers: Since most operators could not be legally licensed during the Weimar period, most active operators also had club-issued shortwave listener identifiers that at least enabled them to legally own receivers. The DASD issued DE identifiers in numerical order, starting in 1925 with DE0001 and reaching around 1932 DE1500, the identifiers play an eminent role in identifying operators. The most important source for this callbook is the "DE list", put together by Ludwig Grünberger after WW2, which also contains calls used for transmission.

\section{Organization of this callbook}

This callbook is subdivided by different time periods between which the call sign system changed. Each time period contains separate alphabetical lists of official ("lis") and unofficial ("unlis") calls. For reference we added also a list of the calls EK4/D4 operators and DE listeners held after 1949. D4 and DA calls in the period from 1933-1948 are included, when known, but not alphabetically listed.

(1) 1927-1930: 1a) EK4xxx/D4xxx - "lis"; 1b) EK4xx/D4xx - "unlis"; 1c) Foreign calls of EK/D stations

(2) 1930-1933: 2a) D4xxx - "lis"; 2b) D4xxx - "unlis"; 2c) Foreign calls of EK/D stations; 2d) Arbeiterempfangsdienst SWLs

(3) 1924-1927: 3a) KA0-KZ9 - "lis"; 3b) Freestyle calls - "unlis"; 3c) Occupational forces; 3d) Official freestyle calls

(4) After 1949: Post-WW2 calls of EK/D stations and holders of DE numbers $(<$ DE6000)

(5) References

\section{Nomenclature}

Note that the DASD coordination was imperfect, the same call was often used by different operators. If necessary, different OPs are distinguished by EK4AL(1), EK4AL(2) etc.

[] References and sources are in square brackets, they refer to entries in the Reference section

? Info from one source but conflicting with others; A likely connection, but lacking thorough confirmation

?? call holder is still unidentified

Brief biographical information can be found in the EK list, when available. For operators who started after 1930 or ended activities before

1927, the biographic information is in the D list or the pre-EK list, respectively.

\section{Acknowledgement}

This research could not have been done without the "DE Liste", put together painstakingly by Ludwig Grünberger (1921-2010), DL3HE, DL6KQ. Two landmark publications about the German history of amateur radio inspired our research, Wolfram Körner's book from 1963 [K] and Leo H. Jung's series of articles, starting in the 1990ies [J]. Some valuable information was provided by Wolf Harranth (-2021), Paulina Petri from the dokufunk.org archive [D] and by Peter Brogl, DK6NP. Much of our research relied on web-accessible digitized records. Biographical dates and later calls of operators could be found in digitized callbooks uploaded to archive.org by KB9MWR. To survey calls on the air from Germany in the 1920ies, we searched sent-in operation reports published monthly in ham radio magazines of that period - the "DX Cluster" of the time. Other important sources were digitized QSL cards in the collections of K8CX, F2VX and SP9EWM, digitized membership lists of OOTC, DIG, chronicles published by DARC districts and local chapters and many other sources encountered in web searches. 
(1) 1927-1930:

1a) Official "lis" calls:

EK4AAA Telegr.-Techn. Reichsamt, Abt. Funkwesen Berlin, KG1, D2AA-D2AY [RL-D2 1935], [M 1927]

EK4AAB Telegr.-Techn. Reichsamt, Abt. Funkwesen Berlin, KG3 [M 1927]

EK4AAC Telegr.-Techn. Reichsamt, Abt. Versuche Berlin, KG0 [M 1927]

EK4AAD Telegr.-Techn. Reichsamt, Abt. Funkwesen Döblitz, KG7 [M 1927], Döberitz Elsgrund [M 1927][P 1928]

EK4AAJ Kyffhäuser-Technikum Frankenhausen, KJ7 [M 1927]

EK4AAK(1) Radio-Vereinigung Gera, KK0 [M 1927]. Ops. Kurt Müller, Liebschwitz, Gartenstr. 1, DE1544M; Helmut Arnold, DE1144F [TUC, E], [G]

EK4AAK(2) Kurt Müller, Liebschwitz/Elster [P 1928][CB1932]

EK4AAL(1) [E] Techn.-Physikalisches Institut der Universität Jena [M 1927], D2CO [RL-D2 1935], Prof. Abraham Esau (1884-1955), Jena, DE0200 [C] - Ops. and students of Esau: Lothar Rohde (1906-1985), DE1431P, DJ5LR, author [CQ-MB 5/32, 7/32, 12/34]; H Schwarz, author in [CQ-MB 1/33], Rhode and Schwarz later founded the company Rhode \& Schwarz, D2DK [RL-D2 1935], [W]

EK4AAL(2) Dr. Ernst Busse (1907-), Jena, DE0147L, later Facharzt f. Chirurgie, Nordhorn [P 1928][CB1932-33]

[Bürkle de la Camp, 1969], author in [CQ-MB 3/30]

EK4AAM Reichsanstalt für Erdbebenforschung Jena, Fahrbare Station auf Auto im Gelände [M 1927]

EK4AAN Staatliche Gewerbeakademie, Schillerstr. 7, Chemnitz, KJ8 [TUC][M 1927]

EK4AAO(1) Funkwissenschaftliche Vereinigung E V, Netzschkau b. Nürnberg, KJ2 [J:1924 Versuchsender][M 1927]

EK4AAO(2) Max Gerstner, Reichenbach [P 1928][CB1932]

EK4AAP(1) [E] Funkwissenschaftliche Vereinigung Plauen Vogtland, D4AAP, KL0, Op: K. Hartenstein, DE0082, EK4HH(2) [F:26][C][U] [M 1927]

EK4AAP(2) EK4HH(2), EK4ML, EK4UU, Kurt Hartenstein, Plauen [P 1928][CB1932]

EK4AAQ

EK4AAR(1)

$\operatorname{EK} 4 A A R(2)$

Deutsche Fernsprecher GmbH, Zschopauerstr. 57-65, Chemnitz [P 1928][TUC]

Funkvereinigung Zwickau, Op: Betriebsleiter: Christoph Schmelzer Jena Fraunhoferstr.1

EK4AAS

Christoph Schmelzer Jena Lichentanne, DE0078 [TUC], D4BIU, [F:28], later physicist at the GSI Darmstadt [TUC] [P 1928][CB1932], author in [CQ-MB 11/31]

EK4AAT F. A. G. Funkapparate-Bau, Hartmannstr. 6, Chemnitz [TUC]

EK4AAU Institut für Schwachstromtechnik der Universität/Technischen Hochschule Dresden, KI9 [M 1927][P 1928]

Dr. Erich Huth G.m.b.H. Berlin, Dip. Ing. Lange Zittau, Bismarkallee 7 [M 1927], D4AAU communicated with CV5BJ, 11/1931 [Krotkofalewski Polski 12/1931]

EK4AAV [E] Politechnikum Friedberg, Hessen, KK9 [M 1927][P 1928][CB1932]

EK4AAW Physikalisches Institut der Universität Giessen, KK3 [M 1927]

EK4AAX

EK4AAY Physikalisches Institut der Universität/Technischen Hochschule Darmstadt [M 1927]

EK4AAZ

EK4ABA

EK4ABB

EK4ABC

EK4ABD

EK4ABE Ingenieur-Akademie Städt. Polytechnikum Oldenburg, KP9 [M 1927]

Verein zur Förderung der Funkwesens, Osnabrück, KP4 [J:1924 Versuchsender]

Radioclub Delmenhorst, KR6 [M 1927][CB1932]

Physikalisches Institut der Universität Greifswald, KF8 [M 1927]

Hanseatische Yachtschule Neustadt i. Holst G.m.b.H. [M 1927]

Motoryacht/Motorkreuzer "Loki”, Hanseatische Yachtschule Neustadt i. Holst G.m.b.H. [M 1927]

EK4ABF(1) [E] Bad. Gesellschaft für Radiotechnik E V, KI2, Op: Wilhelm Doering, Karlsruhe, Zähringerstr. 114, [C][CQ-MB 7/27]

$\operatorname{EK} 4 \mathrm{ABF}(2)$

EK4ABG [E]

EK4OM, Wilhelm Doering, Karlsruhe [CB1932]

Otto Anton Klotz Kühlapparatefabrik (-1953), Heidelberg, DE0550, K-XH, KK7, UHU, K-UHU, K4UHU, D-UHU,

D4UHU, D4BIANCA, EK4OV, D4UVO [J:2], D4OVO, D4ABG, D4BAO 1933 [E], D4AOO 1935, founded 1910

Kühlapparatefrabrik in Heidelberg, Bergheimerstr. 159 [G][C][CB1932], first AM QSO in July 10, 1925

EK4ABH Badische höhere technische Lehranstalt Karlsruhe, KO2, D2CV [RL-D2 1935], [M 1927]

EK4ABI(1) [E] Funkvereinigung Halle, Oswald Kruschwitz, EK4BD, KL4, Op: M. Drechsler, EK4ME [C][M 1927]

$\begin{array}{ll}\text { EK4ABI(2) } & \text { EK4ME, Max Drechsler, Halle, DE0049 [TUC, E], EK4DL [P 1928][CB1932], grinding crystals [QST 4/28 p.72] } \\ \text { EK4ABJ } & \text { Technikum Strelitz, KP1 [M 1927] }\end{array}$

EK4ABK(1) Elektrotechnisches Institut der Ingenieur-Akademie Wismar, KQ1, D2DT [RL-D2 1935], Leiter Dr. Ing. Heinrich [CQ-MB 7/27]

EK4ABK(2) Dr. Ing. Kurt Heinrich (1893-unknown), Wismar, author in [CQ-MB 5/30]

Städtischer Baurat, director of the electrotechnical laboratory of the Akademie Wismar. Member of the freemasons, which lead to first job dismissal in 1931 and his final dismissal in 1935 (freemasons were prohibited in 1935 by the Nazi regime) [CB1932][DL2SWR]

EK4ABL Physikalisches Institut der Universität Marburg, KK6 [M 1927]

EK4ABM Ingenieur Ferdinand Schneider G.m.b.H (1866-1955), Fulda, KL3, Inventor, demonstrated wireless telegraphy with Ferdinand Braun in 1885 (before Marconi), wind power pioneer [M 1927][W]

EK4ABN(1) [E] Kasseler Radio-Club, KQ5, K95?, Op: Franz Noether (-1931), DE0038, EK4KL, D4ABN [CQ-MB 6/27]

EK4ABN(2) Franz Noether (-1931), Kassel, DE0038, EK4KL, D4ABN [CQ-MB 6/27][P 1928]

EK4ABO Gesellsch. v. Freunden der Radiotelephonie und Telegraphie in Marburg (Lahn), KK1 [M 1927]

EK4ABP(1) Südwestdeutscher Radioclub, Frankfurt am Main Niddastr 81, KK5 [M 1927][P 1928] Ops: Johannes Schubert (->1994), Beierfeld Erzgebirge Frankfurt/M, DE0217U, DL9ZG; Werner Hasselbeck, DE0613T [C], D4ABP, Frankfurt/M, 
EK4ABR [E]

EK4ABS

EK4ABT

EK4ABU

EK4ABV [E]

EK4ABW

$\operatorname{EK} 4 \mathrm{ABX}(1)$

EK4ABX(2)

EK4ABY

EK4ABZ

EK4ACA

EK4ACB

EK4ACC

EK4ACD

EK4ACE

EK4ACF

EK4ACG

EK4ACH

EK4ACI(1)

EK4ACI(2) [E]

EK4ACJ(1) [E]

EK4ACJ(2)

EK4ACJ(3)

EK4ACK

EK4ACL

EK4ACM

EK4ACN

EK4ACO

EK4ACP

EK4ACQ

EK4ACR

EK4ACS

EK4ACT

EK4ACU

EK4ACV

EK4ACW

EK4ACX

EK4ACY

EK4ACZ

EK4ADA

EK4ADB(1)

EK4ADB(2)

EK4ADC(1)

EK4ADC(2)

EK4ADC(3)

EK4ADD

EK4ADE(1)
Südwestdeutscher Radioclub, Frankfurt am Main Niddastr 81 [M 1927][P 1928] Op: Gustav Bock, Frankfurt/a. M.-Süd, EK4KB, D4ABQ 1931, [E], D4BFT 3/1933 [V]

EK4AB(1), Ferdinand Bödigheimer, Frankfurt am Main Niddastr 81, DE0069, KK4, KK7 [G] ][P 1928][CB1932]

Rhein. Technikum Bingen (Rhein) Lorch, KJ3, D2CM [RL-D2 1935], [M 1927]

Physikalisches Institut der Schles. Fried.-Wilhelm Universität Breslau, KK8, D2CJ [RL-D2 1935], [M 1927]

Technische Hochschule Breslau, KL9, Op: Herbert Salzbrunn, D4WYG, [J:10] ][P 1928]

Verband der Funkfreunde Schlesiens e. V., Breslau, KK2, KJ4?, [C] OPs: P Kokawke, DE0693G; Paul Strelczyk, DE0762 [TUC], EK4GV [G][C][M 1927][CB1932]

Physikalisches Institut der Techn. Hochschule Hannover, KI1 [M 1927][P 1928][CB1932], D2CT [RL-D2 1935],

Op: Ernst Franzen, Hannover, DE0034; Friedrich Bühring, Steinhude, DE0245K, D4NMK 1938 [U]

Gesellschaft der Funkfreunde Hannover, KI9 [M 1927]

W. Oehlerking, Hannover [P 1928][CB1932], Ober-Telegraphen-Assistant in 1880 in Hannover, founding member of DASD in 1927 [D]

Seismos G.m.b.H. zur Erforschung von Gebirgsschichten und nutzbaren Lagerstätten in Hamm, zwei bewegliche Stationen in der Nähe von Göttingen [M 1927]

Vestische Radiowerke i. Buer (Wstf.) [M 1927][P 1928]

Verein Ostdeutscher Funkfreunde e. V., Königsberg, D4ACA, Ops: Dr. Horst Podszus (-1971), Königsberg Hamburg, DE1319, D4BBA, D4CGA, D4CIJ(2), D4DIE; Hans Wottrich, EK4ES [C][V][G][CB1932]

Hermann Balk Schule Marienwerder i. Westpr. [M 1927]

C. Lorenz AG, Berlin-Tempelhof, KA8, D2BK-D2BO [RL-D2 1935], [M 1927]

C. Lorenz AG, Neuendorf bei Zossen, KC3 [J:1924 Versuchsender]

Deutscher Radio-Klub e. V., D.R.C. Ortsgruppe Ringenwalde Kr. Templin Schloss, KC1 [M 1927]

Funkgesellschaft Eberswalde D.R.C., KC4 [J:1924 Versuchsender] [M 1927]

Op: EK4AY, Otto Schmolinske

Institut der Radiokunde Bremen, KP5 [M 1927]

Deutsche Betriebsgesellsch. f. drahtl. Telegraphie Berlin, Zweigstelle Bremerhaven, D2CI [RL-D2 1935], [M 1927]

Verein der Funkfreunde e. V. Mühlheim, KP6, Betriebsleiter: Dr. Wilhelm Ludolf Schmitz (1899-1973), DE 0072 since

1925, QRT after 1933, [F:23][C], Direktor Institut für Röntgenforschung, Bonn [Lickfeld, 1985]

Dr. Schmitz, Mühlheim [P 1928][CB1932], pure reproduction of phonograph records [QST 5/28 p. 60]

Radiotechnischer Verein "Bergisch Land" Barmen, KP5 [E], Op: O. Knüppel, EK4LY [DE0756 QSL, TUC]

EK4RW, EK4LZ, Dr. Ing. Otto Wilke, Barmen, DE0420H, [P 1928]

EK4RA, v. Allizar, Barmen [CB1932]

Vereinigung der Funkfreunde, Wesel und Umgebung, Op: EK4RU(2) [G][M 1927]

Telefunken Ges. f. drahtl. Telegr. m.b.H. Berlin, KA3, D2BE-D2BJ [RL-D2 1935], [M 1927]

Telefunken Ges. f. drahtl. Telegr. m.b.H. Berlin, KA2 [M 1927]

Telefunken Ges. f. drahtl. Telegr. m.b.H. Berlin [M 1927]

Telefunken Ges. f. drahtl. Telegr. m.b.H. Berlin, Leipzig, KI5 [M 1927]

Telefunken Ges. f. drahtl. Telegr. m.b.H. Berlin, Fürstenbrunn, Wernerwerk, Prüfschuppen, KC0 [M 1927]

Telefunken Ges. f. drahtl. Telegr. m.b.H. Berlin, Fürstenbrunn, Wernerwerk, Telefunken-Prüfwiese, KC0 [M 1927]

Geodät-Institut Potsdam [M 1927]

C. Lorenz AG, Berlin-Tempelhof, KA7 [M 1927]

C. Lorenz AG, Berlin-Tempelhof, KA6 [M 1927]

Dr. Erich F. Huth G.m.b.H. Berlin, KA0 [M 1927][P 1928], Erich F. Huth Signalbau, Berlin, later Hannover

Dr. Erich F. Huth G.m.b.H. Berlin, bewegliche Anlage in der Umgebung von Berlin [M 1927][P 1928]

Deutsche Telefonwerke und Kabelindustrie A.-G. Berlin, KB5 [M 1927]

Physikalisches Institut der Technischen Hochschule Berlin-Charlottenburg, KC6 [M 1927][CB1932], qrv on 20m and 40m [QST 4/29 p.76]

Physikalisch Technische Reichsanstalt Berlin-Charlottenburg, KA1 [M 1927][P 1928]

Labor Dr. Siegmund Loewe (1885-1962), Versuchs-Laboratorium Berlin, Stud. Ing. Helmut/Hellmuth Schulz, KC2 [J:1924 Versuchsender][M 1927] Founded in 1923 with his brother David Ludwig the Loewe Technology Gmbh, later Loewe Opta. He was forced to emigrate in 1938, in 1949 regained possession of some of his company properties. After his death Loewe Opta became part of the Philips group

Stud. Ing. Hellmuth/Helmut Schulz, Berlin Charlottenburg, DE0116F, KD3, EK4ADR? [M 1927][P 1928][G]

Funkverein e. V., Gruppe Elektrowerke A.-G., Berlin, KD9 [M 1927][P 1928], EK4AP? Op: EK4AC [G]

Funktechnischer Verein Gruppe Siemens, Berlin Siemensstadt [CB1932]

Funktechn. Verein e. V., Experimental Station of the Berlin DASD Section, KC8, KB7?,

D4BAF, D4DAS. Ops: Dipl. Ing. Erhard Graff, DE0473F [TUC], EK4CC, D4CCF, D4BAF, DL3CE [E];

Bruno Garnatz, DE1263F, EK4WZ, DL7BG

Dr. Noack, EK4WM(1), Güterbahnhof, Berlin-Schlachtensee [P 1928]

Laboratory of the FTV and DASD, Berlin [CB1932]

Oberingenieur Denes Mihaly, Berlin Lützow, KB2 [M 1927][P 1928]

Funktechnischer Verein, Ortsgruppe Berlin, Potsdamerstr. 4, KB7, Dipl. Ing. Albert Kofes, Berlin, 
[QST 11/27 p.64][F:23/25][M 1927] Op: Prof. Friedrich Trautwein, DE1321, D4CUF 1934, D4HIF 1936, EK4AGE Inventor of the Trautonium

EK4ADE(2) Dr. Albert Kofes, Charlottenburg [P 1928]. He worked for Lorenz AG Berlin and helped built in 1938 the first station in Liechtenstein to relay Radio Beromünster (Switzerland), authored [Kofes, 1938]

EK4ADE(3) Dr. Ing. Friedrich Trautwein (1888-1956), Berlin Zehlendorf, DE1321, DE4115, D4CUF 1934, D4HIF 1936 license revoked in 1939 [CQ-MB4/1939], EK4AGE - Inventor of the Trautonium

EK4ADF(1)

$\operatorname{EK} 4 \mathrm{ADF}(2)$

EK4ADF(3)

EK4ADG

$\operatorname{EK} 4 \mathrm{ADH}(1)$

$\mathrm{EK} 4 \mathrm{ADH}(2)$

EK4ADI [E]

EK4ADJ

$\operatorname{EK} 4 \mathrm{ADK}(1)$ Funktechnischer Verein e. V., Gruppe Osram, Berlin, KB9 [M 1927]

Dr. W. Titius, Berlin-Reinickendorf-West [P 1928]. He was editor of the CQ-MB in 1929 [QST 10/29, p 62]

EK4AL(1), Wolfgang Rach, Berlin Friedenau [CB1932]

"Exploration" Bodenuntersuchungs- und Verwertungs G.m.b.H., Berlin, KB3 [M 1927]

Funktechnischer Verein e. V., Ortsgruppe Berlin-Lichtenberg (früher b. Kohnert), KB6 [M 1927][P 1928]

Funktechnischer Verein Ortsgruppe Lorenz, Berlin Tempelhof [CB1932]

Deutscher Radioklub e. V., Ortsgruppe Schöneberg, e. V. D. R. C., KC7 [M 1927][CB1932]

Ops: F. Kunze DE0188 [TUC]; Rolf Wigand, DE0065, EK4CX(1) [CQ-MB 7/27]

Ferdinand Schuchhardt, Berliner Fernsprech- und Telegraphenwerke A.-G. Berlin SO 16, KD2 [M 1927][P 1928].

Founded in 1886, the company became an Aktiengesellschaft in 1921. ITT corporation acquired the Schuchhardt company in 1929 through its subsidiary International Standard Electric Corporation of New York (ISEC)

$\operatorname{EK} 4 \operatorname{ADK}(2)$

EK4ADL, Dr. Georg Seibt (1874-1934), Fabrik elektrischer Apparate, Berlin-Schöneberg [M 1927][P 1928]

Ing. Otto Kappelmayer (1894-1971), Berlin-Charlottenburg, DE1838F, Funklabor Kappelmayer Berlin-Dahlem,

[G] [P 1928], listed without information in [CB1932]

EK4ADL

EK4ADM

EK4ADN

EK4ADO

Dr. Georg Seibt (1874-1934), Fabrik elektrischer Apparate, Berlin-Schöneberg [M 1927][P 1928], studied with Adolf Slaby at the Technische Hochschule Charlottenburg, started his company in 1910. The company was liquidated in 1950 [W]

Dr. Georg Seibt (1874-1934), Fabrik elektrischer Apparate, Berlin-Schöneberg, bewegliche Anlage [M 1927][P 1928]

“Ahemo"-Werkstätten, Berlin [M 1927][P 1928] company build broadcast receivers, e.g. Ahemo A4

Mix \& Genest, Telephon und Telegraphenwerke, Berlin-Schöneberg [M 1927][P 1928]

Mix \& Genest was founded on 1 October 1879 by the businessman Wilhelm Mix and the engineer Werner Genest in

Berlin-Schöneberg and in 1930 acquired by ITT Corporation, brand name was dropped in 1958

EK4ADP

Mix \& Genest, Telephon und Telegraphenwerke, Berlin-Schöneberg [M 1927][P 1928]

EK4ADQ

EK4ADR?

Deutsche Versuchsanstalt für Luftfahrt, Berlin Adlershof [CB1932]

EK4ADS

EK4ADT

EK4ADA, Helmut/Hellmuth Schulz, Berlin Charlottenburg, DE0116F, EK4ADA, D4ADR, typo in [G]?

EK4ADU(1)

EK4ADU(2)

EK4ADV

Realgymnasum zu Schwelm [M 1927][P 1928]

Radioclub Siegerland e. V., Weidenau (Sieg.), KR5 [P 1928]

Verein der Funkfreunde Schlesiens, Ki4 [J:1924 Versuchsender][M 1927]

Friedrich Dahlke, Liegnitz, Dresden, Fischhausen ab 31.8.1930, DE0379, D4VAA see EK4EG, EK4EB [G]

EK4ADX

EK4ADY

EK4ADZ

EK4AEA

EK4AEB

EK4EB, Friedrich Dahlke (-1943), Liegnitz [P 1928][CB1932]

Berlinisches Gymnasium zum grauen Kloster [M 1927]

Funktechnischer Verein e. V., Ortsgruppe Wernigerode (Harz) Gymnasium, KI0 [M 1927][P 1928]

Magdeburger Versuchsschule [M 1927]

Staßfurter Licht- und Kraftwerke A.-G. Staßfurt, KM3 [M 1927][P 1928]

Funktechnischer Verein e. V. Bezirksgruppe Magdeburg, KP0 [J:1924 Versuchsender][CB1932]

Junkers-Flugzeugwerke A.-G. Dessau, KN1 [M 1927]

Junkers-Flugzeugwerke A.-G. Dessau, Flugfunkstelle, KN2 [M 1927]

EK4AEC

EK4AED

EK4AEF

EK4AEG

EK4AEH

EK4AEI

EK4AEJ

EK4AEK

EK4AEL(1)

EK4AEL(2)

EK4AEM

EK4AEN(1)

EK4AEN(2)

Junkers-Flugzeugwerke A.-G. Dessau, Flugfunkstelle, KN3 [M 1927]

Junkers-Flugzeugwerke A.-G. Dessau, Flugfunkstelle, KN4 [M 1927]

Junkers-Flugzeugwerke A.-G. Dessau, Flugfunkstelle, KN5 [M 1927]

Junkers-Flugzeugwerke A.-G. Dessau, Flugfunkstelle, KN6 [M 1927]

Junkers-Flugzeugwerke A.-G. Dessau, Flugfunkstelle, KN7 [M 1927]

Junkers-Flugzeugwerke A.-G. Dessau, Flugfunkstelle, KN8 [M 1927]

Junkers-Flugzeugwerke A.-G. Dessau, Flugfunkstelle, KN9 [M 1927]

Junkers-Flugzeugwerke A.-G. Dessau, Flugfunkstelle, KN0 [M 1927]

Deutsche Betriebsges. F. drahtlose Telegr. m.b.H., Hamburg, KI3 [M 1927][P 1928]

EK4AEZ, Flugschule Staaken bei Berlin Spandau [CB1932]

Phys. Staatslaboratorium der Universität Hamburg, KL2, D2CS [RL-D2 1935], [M 1927][P 1928]

Radioröhrenfabrik G.m.b.H., Vulkan, Hamburg, KM6 [M 1927]

EK4AFC, Ernst Ramcke, Hamburg [P 1928][CB1932]

EK4AEO(1) [E] Hamburger Radioklub e. V., KI6, Betriebsleiter: Dr. Richard Wohlstadt, Hamburg, DE0153 [TUC], [G]+ [M 1927][CB1932], photo in [CQ-MB 6/30], Senderleiter Carl Kerger, DE0106J [TUC] [CQ-MB 2/27][D]

$\operatorname{EK} 4 \mathrm{AEO}(2)$

EK4AEP(1)

EK4AEP(2)

Fritz Hoffmann, Altona Berlin, DE0664J [G], [P 1928]

EK4AEQ(1) [E] Deutscher Radioklub e. V. Cottbus, KQ7 [E], Op: EK4BR, [M 1927][P 1928]

EK4AEQ(2)

EK4AER(1)

$\operatorname{EK4AER(2)}$

EK4AES

EK4BR, H. Krautzig, Kottbus [CB1932]

Deutscher Radioklub e. V. Ortsgruppe Fürstenwalde, KB1 [J:1924 Versuchsender], [M 1927][P 1928]

Allg. Transportanlagen A. G., Leipzig [CQ-MB 10/32]

Physikalisches Institut der Universität Leipzig, KI5 [M 1927] 
EK4AET Technikum Mittweida, KK4 [M 1927][P 1928], D2DA [RL-D2 1935], [CQ-MB 10/32],

EK4AEU(1) Radiovereinigung Leipzig e. V., Op: Karl Winkler, Leipzig, Wächterstr. 24 (Laboratorium), DE0194 [E], D4AEU [C] [CQ-MB 7/27][U][F:26]

EK4AEU(2) Karl Winkler, Leipzig, DE0194U [E], [P 1928][CB1932]

EK4AEV Technische Hochschule Braunschweig, KI7 [M 1927]

EK4AEX(1) Physikalische Werkstätten, Göttingen, KJ6 [M 1927][P 1928]

EK4AEX(2) Flughafenfunkstelle Berlin Tempelhof, AEX, D4AEX, DDX, DDDX,

Op: Franz Otto Baer, Halle/S., Tempelhof, DE1566L/F [TUC], [G][C]

EK4AEY [E] Funkverband Braunschweig, KQ2 [M 1927] D4AEY, Ops: Hermann Siems (-1937), DE0359, D4PSQ, D4CMA, built x-tal Driven 5m transmitter in 1931; K. Winkler, DE0194 [CB1932]

EK4AEZ

EK4AFA(1)

$\operatorname{EK} 4 \mathrm{AFA}(2)$

EK4AFC [E]

EK4AFD

EK4AFE

Flugschule Staaken bei Berlin Spandau, EK4AEL, D4AEZ, D007 [C]

EK4AFF

EK4AFG

EK4AFH

EK4AFI

EK4AFJ

$\operatorname{EK} 4 \mathrm{AFK}(1)[\mathrm{E}]$

EK4AFK(2)

EK4AFL

EK4AFM

Deutscher Radio Club e. V. Berlin-Charlottenburg, KD6 [M 1927] Op: Curt Lamm, DE0152, EK4CL, D4AFA, OZ2CL since 1933 [E]

EK4CL, Dr. Curt Lamm, Berlin [P 1928][CB1932], author of [QST 5/28 p. 60, 6/28 p. 68, 7/28 p.72]

Ernst Ramcke, Altona, Carolinenstr. 24, DE0657J [G][CB1932]

Telefunken, Berlin [CB1932]

Dr. P. Lertes, Frankfurt a. M. [CB1932], author in [Günther, 1925], [Lertes, 1926]

Gewerbeschule Köthen (Anhalt), Op: Prof. Dr. Heinrich Wigge, D4AFF [CB1932], [D]

Aeronautisches Observatorium, Lindenberg Kr. Beeskow [CB1932]

Ingenieursschule Altenburg (Thüringen) [CB1932]

Deutsche Seewarte, Hamburg am Stintfang, D2CR [RL-D2 1935], [CB1932][CQ-MB 7/27]

Höhere Maschinenbauschule, Neustadt-Glewe [CB1932]

EK4AFR

EK4AGC

EK4AGE

EK4AGN

EK4DAO

EK4DBA [E]

EK4DBD

EK4DBS

EK4DBW

EK4DCF

EK4DDC

EK4DDF

EK4DEA

EK4DFK

EK4DFZ

EK4DKA [E]

EK4DKB

EK4DKF [E]

EK4DOA

EK4DOB

EK4DOC

EK4DRT

EK4FAA

EK4FAB

EK4HBL

EK4HFL

EK4HLF

EK4KAD
EK4AF(1), Pasewaldt, Döberitz bei Berlin [C]

Telefunken, Berlin [CB1932]

Askania Werke, Berlin Friedenau, C. Bamberg [CB1932]

H. Brinkmann (- 1956), Telegrapheninspektor, Bielefeld, Uhlandstr. 5, DE1228, D4AFM, D4BSK [E], D4DSK, D4IOH

[E], D4BBJ?, D4ICH, DL3EZ, Hermann Brinkmann, Bielefeld, Freiligrathstr. 2, CB 1951 [G][C]

Telefunken, Berlin [CB1932]

Erich Winzer, Berlin Lottumstr. 13, DE0541 [E], [G]

Prof. Dr. Friedrich Trautwein (1888-1956), DE1321, DE4115, D4CUF 1934, D4HIF 1936 license revoked in 1939

[CQ-MB4/1939], D4ADE, Hochschule für Musik, Berlin Charlottenburg, built 1921-1923 the first broadcast transmitter for

Deutsche Post, pioneer of electronic music, inventor of the Trautonium, teacher at music conservatory Berlin

Charlottenburg, collaboration with colleague Paul Hindemith [G][CB1932]

EK4BY, Josef Brey [V]

Fritz Scheuering, München, DE1532P, Heer [F][G], maybe the author of [Müller \& Scheuering, 1944]

Hugo Fagien (-1953), Versuchsstation Königsberg, KPl, DE0673, EK4MCA, EK4EU, D4FUE, D4BOA, D4SDA, D4SDF, died 1953 in Berlin, 1925 Techniker Ostmarken Rundfunk-AG (ORAG), D4SDA [C], [P 1928]

qsos with USA (120W) [QST 4/29 p.76]

EK4DB, Fritz Schnepel (-1979), Königsberg, Pr., D4DBD [C][CB1932]

Gustav Dybus, Königsberg Hannover (Heer), DE0674B, KW5, K4DOS [G], listed as Günther Diebus in Kalthof

Königsberg in [P 1928]

EK4ES, Hans Wottrich

Fritz Weber, Reichswehr, Berlin, DE1861, D4DCF 1932 [J:8], probably founder of Fritz Weber Metallwaren und

Laternenfabrik, Berlin, NSDAP member

EK4BY, EK4KZA, Josef Brey, Heer [F]

Georg Ewert, Mowawes Mühlhausen Potsdam Perleberg, DE2042C, D4DDF, D4KQD, D4HZL ?,

division Rathenau Heer RR4 [F][G]

Heidenfelder, Stuttgart Bad Cannstatt, DE0011, D4DEA, D4DKE, Mechaniker-Meister [G]

EK4DKF, Oscar Gruber, Stuttgart [C], EK4LG

A. Neunert, Berlin, DE1742F, Heer [F][G]

Heidenfelder, Stuttgart Cannstatt, DE0011N, Mechaniker Meister, Heer [G][C][F][CQ-MB 7/27]

Nachrichtensoldaten der Reichswehr, Kassel, D4DKB, Frhr. von Lersner, verantw. Von Uckermann, [memory of DL1AP]

Oscar Gruber, Münster, Stuttgart Cannstatt, Potsdam, DE0678, EK4LG, EK4DKF, D4LGL, D4BQH, D4RRH [G][F],

listed as Oskar Schweingruber, Cannstatt in [P 1928]

EK4DAO, Fritz Scheuering, München, DE1532P [G][CB1932]

EK4KUA, Hans Link [V]

Adam Schönberger, München, DE2081P, Heer [F][G]

$\mathrm{Ph}$. Eckhardt, Heer [F]

Magdeburger Branddirektion, Magdeburg-Neustadt Feuerwache [P 1928]

Magdeburger Branddirektion, Magdeburg-Neustadt Feuerwache [P 1928]

[CQ-MB 7/27] ??

Hochschule für Leibesübungen, Berlin [P 1928]

Hochschule für Leibesübungen, Berlin Spandau [CB1932]

Karl Andrae (->1995), Berlin Halle Lichtenberg, Heer, DE1565L, D4HAD, D3AMF, DM2ABH [CB 1957],

DM2ADO [CB1959], DM2KAD, Y21DO, DL7UDJ [F][G] 
EK4KBL

EK4KBU

EK4KGL

EK4KHF

EK4KKA

EK4KMA

EK4KMC

EK4KQB

EK4KQC

EK4KQD [E]

EK4KQE

EK4KQF [E]

EK4KQG

EK4KUA

EK4KUB

EK4KUE

EK4KUR

EK4KZA

EK4LAA

EK4LAB

EK4LAC

EK4LAD

EK4LAJ

EK4LAX

EK4MFM

EK4SAR

EK4UAA

EK4UAB(1)

EK4UAB(2)

EK4UAC(1)

EK4UAC(2)

EK4UAD

EK4UAE

EK4UAF(1)

EK4UAF(2)

EK4UAG(1)

EK4UAG(2)

EK4UAH [E]

EK4UAI

EK4UAJ

EK4UAK

EK4UAL

EK4UAM(1)

EK4UAM(2)

EK4UAN(1)

EK4UAN(2)

EK4UAO(1)

EK4UAO(2)
EK4KBU, W. Kilian, Niederzwehren Kassel [C][CQ-MB 7/27][P 1928]

W. Kilian, Niederzwehren b. Kassel, EK4KBL, Heer [F][CB1932]

[CQ-MB 7/27] ??

[CQ-MB 7/27] ??

EK4BY $[\mathrm{G}]$

Erich Tripmacher, Hannover Sprendlingen, Heer, D4KMA, EK4TP, D4RTA, D4CMJ 1928 [V], DL3DQ(1) [CB 1949],

[F][CB1932]

EK4QU, Rudolf Asbach, Heer [F]

Paul Reschke, Osterode Lager Lechfeld, DE1737A, Kavallerie [F][G]

E. Sakschewski, Kavallerie [F]

EK4DDF (Reiter?), Potsdam, [listed as EK4KOD in C], Kavallerie [F][G]

Ltn. Schrottler, Kavallerie [F][CB1932]

Hans Schleifenbaum, Kavallerie [F], DEM1721, D4CBC, D4CAM [E], D4CAL(2) [E], D4CAR, DL1YA [E], [G],

BND agent alias "Schleyer, Stein" [Lecorte, 2015]

M. Jakob, Kavallerie [F]

Hans Link, Bamberg Cannstatt, DE1163R DE2133R [QSL 1935, E], D4ULI [E][V], Kavallerie, D4KUA [E], EK4DOB, EK4KUR? [F][G][CB1932]

Heinrich Wurz, Bamberg Langensalza, DE1167R, Kavallerie [F][G]

Otto Rabethge (-1976), Parchim Stettin Kiel, DE1785V/B, D4HUB [E], D4CLJ, D4CGT(2), DL1GN [E], Kavallerie [F][G] EK4DOB

EK4BY, EK4DDC, Josef Brey, Heer [F][CB1932]

Rolf Ehrhardt (-1955), Pillau Cuxhaven Wisch bei Hamburg, DE1715A, D4CDA, Marine, DJ1HT in Lahr [CB1953-1955]

served 1934 on the Kreuzer Karlsruhe and visited W7APS [Radio, July 1934], 1941 on submarine U770 [F][G]

Bodo Kiesel, Stralsund Hannover Kiel Warnemünde, DE1728V, D4HIF, D4BRJ(2)?, D4BRV(2) [E], Marine [F][G]

Werner Arnold, Pillau Kiel, DE1776A, Sondergruppe M, D4CFA, DL1OB, Marine [F][G]

Josef Stratenschulte, DE1762A, Sondergruppe M, D4CHA, Marine [F][G]

Hugo Wördemann (1915-1999), Cuxhaven Temecula, DE1857J, D4BSS, D4BZJ [E], D3LAM, WB6CJG, K6TE [G]

[CQ-MB 6/27, 7/27] ??

[CB1932] ??

EK4AH(1), Julius Kron (typo Kroom), Saarbrücken [P 1928]

Benediktinerstift St Stephan, Augsburg, KV9 [M 1927][P 1928][CB1932]

Radioverein Coburg, KW5 [M 1927] Op: Walter Dieminger, DE0240, EK4VF, K4UAB, D4UAB, D4UAK, DL6DS [E],

Author in [DASD, 1931][C]

EK4VF, Walter Dieminger, Amberg, DE0240 [P 1928][CB1932]

Dr. Ing. Otto Papp, Burghausen Mückenberg, DE0001 [TUC], DE1824P, KW1, D4UAC [E], D4QCP, D4BFP,

D4QZU, D4QZP [CQ6/1937], D3CRT [G], [M 1927][P 1928]

Gesellschaft für Missionsfunk, Niederndobrach near Kulm, D2BD [RL-D2 1935], Op: Robert Wunder,

[CQ-MB 7/27][CQ-MB 12/34]

Dr. Max Wilhelm Friedrich Dieckmann, a. o. Prof. a. D. Technischen Hochschule München, Gräfelfing, KV1 [M 1927],

works on picture transmissions [QST 7/28 p.72], [P 1928]

Versuchsanlage der D.R.P. Kochel, RPD München Kochel D2AZ [RL-D2 1935], [M 1927][P 1928]

Leonhard Frohn (- 1983), DE0007, München, St. Poelten, Wien, UOXR, UOXE, OE001, DL3PK [G]

Versuchsanlage der D.R.P. Bewegliche Anlage [M 1927][P 1928]

"Telefunken" Zweigstelle für Süddeutschland, München, KW2 [M 1927][P 1928]

Dr. Hans C. Deckel, Solln, DE1568V/P, D4REZ, D2DH [RL-D2 1935], D3BMP [E], Friedrich Deckel AG [E]

Viktor Gramich, cand. Ing., Versuchsstation Murnau, KW3 [M 1927] in 2/1929 first contact on 10m with Australia using 25 Watts [QST 5/1935, page 11]. Op: EK4UH, Dipl. Ing. Viktor Gramich [C][CQ-MB 7/27][CB1932], WAC diploma 1927 [CQ-MB 2/34], [P 1928]

Süddeutscher Radioklub, München, KW6 [M 1927][P 1928][CB1932]

EK4UQ, Dipl. Ing. L. v. Türkheim, München, KW7, D2DE [RL-D2 1935], [M 1927][CB1932]

Oberleutnant Fritz Behringer, Gudrunstr. 9, München, DE0340 [C][M 1927][V][CB1932]

Anton Freiherr von Massenbach (1899-), München, KW1?, KW9, EK4UAM [M 1927][CB1932] Born in Lindau, he participated in WW1 in a signal corps. After the war he studied at the Polytechnikum Cöthen and joined Lorenz AG in 1926. There he developed air-born radio systems and got his pilot license in 1932 . He led the development of

Communication systems for the German airforce and war production of Lorenz radio equipment at other companies. Got procura in 1939 and led the Lorenz branch office Munich after WW2 [Lorenz AG Festschrift, 1955]

Süddeutsche Telefonapparate \& Drahtwerke A.-G., Nürnberg, KV7, D2DL [RL-D2 1935], [E][M 1927]

EK4UAL, Anton Freiherr von Massenbach (1899-), Antonienstrasse, München [P 1928]

Süddeutscher Radio Club, Verw. Bez. Nord in Nürnberg [M 1927][P 1928]

Ops: Fritz J. Maas, Nürnberg, Felsenstr. 20 [E], D4UAN Hans Bauer, EK4VQ, D4UAN, Fürth [C][V]

EK4VQ, Hans Bauer, "Funker Schupo", DL1DX [E] Fürth [C][V][CB1932]

Fränkischer Radio-Club Würzburg, Kv8, D4UAO, Ops.: Walter Dieminger, EK4VF, Karl Rückert, EK4VK(2) [E],

[P 1928][Harranth, 1996]

Erziehungsanstalt Ettal, Pater Emanuel Haiss (1895-1977), Kloster Ettal, DE1342P, D4IOI, D2DF [RL-D2 1935], DL2PE;

2nd OP: Heinrich Haffmann, München Ettal Canada, DE1334P, D4EZU, D4BTP [G][V][CB1932] 
EK4UAP Städt. Handelsschule Augsburg [M 1927]

EK4UAQ Überlandwerke Niederbayern, Landshut [M 1927]

EK4UAR Höhere Technische Staatslehranstalt, Nürnberg, K4UAR [E][M 1927]

EK4UAS [CQ-MB 7/27] ??

EK4UAW EK4UF, F. M. Daser [CB1932], other op: EK4UL(3) [CB1932]

EK4UAX

EK4UAZ

EK4XAA

EK4XAB

EK4XAC

EK4XAD

EK4XAE

EK4XAF

EK4XAG

??, Op: EK4VZ

EK4UL(3), Johann Kagermeier (Tuntenhausen), D2DG [RL-D2 1935], [CB1932]

Elektrotechn. Institut der Technischen Hochschule Stuttgart, KY1, D2DW [RL-D2 1935], [M 1927][P 1928]

Physikalisches Institut der Technischen Hochschule Stuttgart, KZ3 [M 1927][P 1928]

Luftschiffbau Zeppelin G. m. b. H. Friedrichshafen [P 1928], Zeppelinwerft Flughafenfunkstelle Friedrichshafen (Bodensee) [CB1932]

EK4YAA(1)

Radiokosmos-Abteilung der Franck'schen Verlagsbuchhandlung Stuttgart, KZ0, D2DX [RL-D2 1935], [M 1927][P 1928] [CQ-MB 7/27]. Op: Nestel EK4LV [QST 7/28 p.72]

Radiowerk A. Laukart, Trossingen, KY9 [J:1924 Versuchsender][M 1927][P 1928]

Telegraphen-Werkstätten der O.P.D. Stuttgart [M 1927][P 1928]

Dornier Metallbau, Friedrichshafen (Bodensee) [CB1932]

Oberdt. Funkverband e. V. Stuttgart, KY4 [M 1927] Op: Rolf Formis (1894-1935), Stuttgart, KY4, K4YAA, D4YAA, [C] [CQ-MB 7/27], SUERAG, Schwarze Front, killed by SS in 1935, see [K][W]

EK4YAA(2) Rolf Formis (1894-1935), Stuttgart, KY4, K4YAA, D4YAA, [C][CQ-MB 7/27], SUERAG, Schwarze Front, killed by SS in 1935 , see [K][W], [P 1928]

EK4YAA(3) EK4XM, Dipl. Ing. Rudolf Mertz [CB1932]

EK4YAB(1) Funkverein Stuttgart im OFV, KY5 [M 1927] Op: Fritz Sabrowsky, Stuttgart, DE0101N, Ky5, D4YAB [F:23/26][CQ-MB 7/27][CB1932]

EK4YAB(2) Fritz Sabrowsky, Stuttgart, DE0101N [P 1928][CB1932]

EK4YAC Funkverein Ulm a. Donau im OFV [M 1927] Op: EK4XU(2), P. Windelschmidt, Ulm a. Donau, DE1128, Bleicherwalkstr. 11, D4YAC [C], [P 1928]

EK4YAD $\quad$ Funkverein Göppingen im OFV, KY7 [M 1927][CQ-MB 7/27][P 1928]

EK4YAE(1) Funkverein Rottenburg im OFV, KY8 [M 1927] Dipl. Ing. Rudolf Horkheimer (1894-1982)

EK4YAE(2) [E] Dipl. Ing. Rudolf Horkheimer (1894-1982), K4YAE, CP6XF, DK5GA

[E], (phonie) [CQ-MB 7/27] chief engineer at Rectron, a tube company in Berlin associated with Philips, Holland. He Emigrated 1938 to Bolivia, his parents were killed. He returned in 1958 to Germany [Kienzle, 2008][DK5GA letter], 1st crystal controlled TX [QST 11/26 p.48, QST 1/27 p.53], pure reproduction of phonograph records [QST 5/28 p. 60], heard by DE0714 [QSL to EK4ABI, TUC]

EK4ZAA Technische Hochschule Danzig [P 1928], YM4ZAA in 1931[E], Op. K. Martin

EK4ZDG EK4ZO

EK4ZDF Dipl. Ing. Hans Heyden, Danzig-Langfuhr, DE0171, YM4ZDF, YM4AB, LG 17 founded 1933, member Verband Deutscher Elektrotechniker Danzig [VDE Listing, 1925] [G][S]

EK4ZZZ [E] Elektrotechnisches Institut an der Technische Hochschule Danzig, KDZ1, Ops.: W. Schade, DE0085Y, DJ3ZY?? [G]; Dipl.-Ing. Hellmut Werrmann, member Verband Deutscher Elektrotechniker Danzig, worked for Siemens A.-G. 1934-1943 [VDE Listing, 1925][C][E][S] 


\section{1b) Unofficial “unlis” calls:}

EK4AA Joachim Börner (-1994), Berlin, DE0948, D4RAA, EK4WA, EK4WN, D4BKF, D4BIF, D4GNW [E], DL7AT [G] [CQ-MB 7/27]

EK4AB(1) Ferdinand Bödigheimer (1895-1986), Frankfurt, DE0069, Kk4, Kk7, EK4KG, EK4ABR, D4BAT, D4ATT, teacher Volksschullehrer, author, soldier in WW1 1915-1918, Wachtmeister der Reserve, 1934 SA (Übernahme Stahlhelm), Abwehr-Funkstelle XII "Eiserne Hand" bei Wiesbaden, WW2: Agentenfunkleiter Ost, 1946 Organisation Gehlen - BND, Deckname "Banner" [Müller, 2017][C][CQ-MB 6/27], bio in [CQ-MB 7/1937]

$\operatorname{EK} 4 \mathrm{AB}(2)$

$\mathrm{EK} 4 \mathrm{AC}(1)[\mathrm{E}]$

$\mathrm{EK} 4 \mathrm{AC}(2)[\mathrm{E}]$

EK4AE

EK4AF(1) [E]

EK4AF(2) [E]

EK4AG(1)

EK4AG(2)

EK4AH(1)

EK4AH(2)

EK4AH(3)

EK4AI(1)

EK4AI(2)

EK4AJ [E]

EK4AK

EK4AKÜ

EK4AL(1) [E]

EK4AL(2)

EK4AM [E]

EK4AML

EK4AN(1) [E]

EK4AN(2)

EK4ANS

EK4AO

EK4AP [E]

EK4AQ [E]

EK4AR(1)

EK4AR(2)

EK4AS

EK4ASA

EK4AT

EK4AU [E]

EK4AV

EK4AW [E]

EK4AX

EK4AY [E]
Albert Rinke, Berlin Gladow, DE0860F, D4GBA(1) [G]

EK4EY, Dr. Herbert Schulz (-1984) [G]

Hans Lamprecht, Berlin, DE1208F, D4RCA, D4BZF, D4GVF [G][C]

Werner Burmeister (->1994), Berlin, DE0946 [E], D4GEA, D4BBF [E], DL7UB [G] [CQ-MB 7/27],

author in [CQ-MB 11/31]

EK4CC, Dipl. Ing. Erhard Graff (-1989), Döberitz bei Berlin, DE0473F [TUC], EK4AFK, EK4BA, DL3CE [CB1962]

[CQ-MB 7/27][G]

EK4RU(1), Karl Mälzer

Hans Heineck, Berlin Jörsfelde, DE0448 [E], Op at EK4ADK [G][DE0448 QSL 1928], drew cartoons CQ-MB 1/30, 3/30, left DASD 1938

Heinz Schulz, Berlin, DE0812F, D4WGA, emigrated in 1932 to the UDSSR [G]

Julius Kron (-1957), Berlin, DE0181, D4AH, D4IAH, EK4SAR, D4SAR, D4IHA, D4BFF, D4GEF [D][G]

died 1957 in Caracas, [J:7], author in [CQ-MB 3/30, 5/30, 6/31]

Otto Haugwitz, Hannover Dieburg/Weser, DE0881K, D4PHD, D4BMK [E], D4JSK [G]

$\mathrm{EK} 4 \mathrm{CH}$

Fritz Köpper, Berlin Köln, DE0995I, D4GAI, D4FKF [G]

EK4HZ

EK4QY, Hans Reifenberg, Berlin Grunewald [C][G]

EK4BY, Josef Brey, EK4DDC, EK4KZA [CQ-MB 7/27][G]

[CQ-MB 7/27] ??

Wolfgang Rach (- 1963), Berlin Schwerin, DE0714F [TUC], D4AL, D4KLA, D4ADF, D4CFF [E], D4FFF [CB1935], DM2ABB [G][E] (1st qso: 6.4.1928)

H. Honold, K4AL 1926 Stuttgart Schwäbisch Hall, freely invented [C][K][CQ-MB 6/27][E]

August-Clemens Hofmann ( $\sim 1960)$, Berlin Straubing, DE0713, D4LMA, D4COF [C], DL3EY [CB1951] August Cl.

Hofmann, Straubing, Bahnhofstr. 8, [E][V], author in [CQ-MB 6/30]

[CQ-MB 7/27] ??

Max Vantler (-1959), Berlin Oranienburg, DE0605, EK4QV, D4PVQ, D4JWF, D4WAC, D4RDH, D4BWK [F:26][C][E], grinding crystals [QST 4/28 p.72][U], heard by W2JD [QST 4/1929 page 84], Author, (Kaufmann, until 1903: Vorstand

Panzer Akt. Ges. für Geldschrank, Tresorbau und Eisenindustrie, Berlin)

EK4TA, Adolf Hohenner

[CQ-MB 7/27] ??

Otto Laaß (1896-1969), Berlin, DE0346, D4SOA, [G][K], Director of Telefunken Public Relations [Berlin-Chronik, 2015]

Carl F. A. Pailler, Berlin (1904- 1985), DE0161, qsl from 1927 [E] says he operated from the airplane as xEK4AP, DC7CF [E][CQ-MB 7/27][G] Organizes in 1947 the WBRC district of Sachsen, is caught, stripped of his property and convicted to 31 years forced labor. Escaped to West Berlin in 1954 [D]

Fritz Zegenhagen, Berlin Charlottenburg, DE0754F, D4MAQ [G][E]

Alfred Schumacher, Berlin, DE0650 [G], maybe identical with DJ4PH Mannheim [CB1959->1995]

EK4AH, Julius Kron, DE0181, [CQ-MB 7/27]

Alfred R. Schlosshauer (1911-1988), Berlin Borg (Ostprignitz) Heidelberg Arogno, DE1296O/H, D4OSA [E], D4BJF

[CB1936], D4GJA, D4GJO [E], D4GJC [E], DJ4WN [E], HB9AWV, HB9EWV?, DF4UN? OOTC1885 [G][V][E]

EK4DBD, EK4DB, Fritz Schnepel

Dr. Gerhard Jacoby (1911-2002), München Andechs Berlin Germering, DE0792F [C], D4BTA, D4BTE,

DL3ME [E], [OOTC1333]

Rudolf Romeike (1912-1972), Berlin, DE0300, EK4UA [E], D4ZUA, D4WUA, D4ZUF, D4FXF [CB1935], DA5UA, DL1QW [E], DK4AU [1948 and CB1972][C][CQ-MB 7/27][G][DJ4ZC][E][CQ-MB 6/27], author in

[CQ-MB 4/30, 12/30, 7/31], crystal controlled transmitter [QST 6/29 p.67][Harranth, 1996]

Rudolf Urtel (1906-1954), Berlin, D4AV, [F:26], Developer of TV technology Telefunken [W],

heard overseas [CQ-MB 11/30]

Berlin, 4AW, [C], OPs: ex EWai es EWbf [E]

YL Station, Berlin, mentioned on EK4AP qsl from 1927 [E] ??

Otto Schmolinske (1895-1985), Berlin, DE1116F, DE5000F, D4VAY, D4AEZ? [D], D4BEF, D4ACF, member FWGM [G][E]

Participated in the battle of Jutland (1916) and the Kapp Putsch (1920). Engineer for Lufthansa 1923-1928.

Kapitänleutnant z. See, ab 1934 Freiwillige Wehrfunk Gruppe Marine (FWGM) [D], author in [CQ-MB 4/30], heard overseas [CQ-MB 12/30]

EK4AZ Dr. Herwig Bender (- 1990), Berlin Karlsruhe, DE0640F, D4SZA, D4XSF, D4FDF, DK2FR [U][G]

EK4BA(1) Willi Schenk, DE0318, D4FAB, [G]

EK4BA(2) [E] EK4AF, EK4CC, Erhard Graff 

[OOTC1996][J:FA9/98]

EK4BB(2) Fritz Schott (-1980), Baden Baden, DE0041 [TUC], DK6AA, DL6IR [G]

$\mathrm{EK} 4 \mathrm{BB}(3)$

EK4BD

EK4BC

EK4BE

EK4BF

$\mathrm{EK} 4 \mathrm{BH}(1)[\mathrm{E}]$

\section{$\operatorname{EK} 4 \mathrm{BH}(2)$ \\ $\mathrm{EK} 4 \mathrm{BI}(1)[\mathrm{E}]$ \\ EK4BI(2) \\ EK4BJ [E] \\ EK4BK(1) \\ EK4BK(2) \\ EK4BL(1) \\ EK4BL(2) \\ EK4BM(1) \\ EK4BM(2) \\ EK4BN \\ EK4BO [E] \\ EK4BP(1) \\ EK4BR [E] \\ EK4BS}

EK4BT

EK4BU(1)

EK4BU(2)

EK4BV

EK4BW(1)

EK4BW(2)?

EK4BW(3)

EK4BX(1)

EK4BX(2)

EK4BY [E]

EK4BZ

EK4CA

EK4CAT

EK4CB [E]

EK4CC [E]

EK4CD

EK4CE

EK4CF

EK4CG [E]

$\mathrm{EK} 4 \mathrm{CH}$ near Stralsund, signs "Baby" [E]

EK4MI, Oswald Kruschwitz, heard by PL198, 11/1931 [Krotkofalewski Polski 12/1931]

Gerhard Petrich (->1994), Spremberg Beelitz Mosbach, DE0817C [E], D4BCB, D4BIC, D4DMC, DJ2PM [G]

Heinz Jäger (->1994), Frankfurt Oder, Bremen, DE1174C, D4FEB, D4BHC, D4DLC [E], DL1UE [E], [G],

D4FEB heard overseas [CQ-MB 6/31]

Günther Sinapius (-1944), Spremberg, DE0816C, D4BFB, D4BLC, D4DQC [G]

Ehrenfried Scheller (-1978), Cottbus, DE0912C, D4BH, D4IHB, D4BQC, D4DYC, D4DYL in 1936, DM3KCJ,

DM2AEJ [CB1958][C], Schreiben des Amtsgerichts Cottbus vom 27.7.1932: „Strafsache gegen den Studenten Ehrenfried

Scheller in Jena: Wegen Vergehen gegen das Gesetz über Fernmeldeanlagen [ . . . ergeht Strafbefehl in Höhe von 20RM

Oder Gefängnisstrafe von einer Woche.“ Das Universitätsamt vermerkt am 9.11.1932: „Der heute erschienene stud.

Scheller ist von Sr. Magnifizenz entsprechend vermahnt worden.“

EK4CH, Ernst Hofmann

Heinz Emde, Cottbus, DE1063C, D4BI, D4RIB, D4BIB, D4BVC [G][C][V],

author of "Begegnungen auf dem Südatlantik" in [CQ-MB 11/1935], was listener in 1935 [U]

EK4KC? [G]

Hermann Schulz, Spremberg, DE1084C, D4EJB, D4BJC, D4DNC [G][C]

Fritz Rinck, Cottbus Erfurt, DE1171G [C], EK4RH?, EK4BP?, D4MBP, D4BFI, D4DJC [E], [G][CB1936]

later EK4XB

Emil Böhm (->1995), Berlin Stettin, DE0983B, D4BLD, D4BEB, DL7CB [G][CQ-MB 7/27]

Karl Heinz Kollmorgen (1909-1999), Nowawes Berlin/Zehlendorf, DE1211C, D4CBA [E], D4EBW, D4BEC [E], DL7DZ [E], on the air since 1930, [G][CB1955]

Dr. med. Heinz Thiem (-1962), Goslar Berlin Eberswalde, DE1179, D4RMB, D4BNC [E], D4DSC, D4DSF, DL1TU [E], [G][C], D4RMB activity reported [CQ-MB 11/30]

EK4MM, [CQ-MB 7/27]

Gustav Karl Metzner, Cottbus Spremberg Heidemühle, DE1187, D4ZNB, D4DPC, D4BKC [G]

Fritz Janssen, Alttrebbin/Oderbruch Philipatal ueber Potsdam, DE0856C [C], D4VOB,, D4BMC, D4DRC [E], D4DYL, near Berlin, [C][G], D4VOB heard overseas [CQ-MB 3/31]

EK4MA, Werner Pilz (-1976), Syrau-Plauen, D4MPB [E] 1930, [E][U][G]

Hans Krautzig, Cottbus Dresden, DE0761C [E], EK4AEQ, D4BRB, D4AEQ [C][G]

Helmut Grützmacher, Stettin Bayreuth, DE0978B, D4FSB [E],[G], D4BAB, D4ABB,

D4FSB heard overseas [CQ-MB 6/31]

Wolfgang Böhm, Stettin Lübeck, DE1224J, D4ETB, D4FGB, D4BDB [G]

Günther Breitenreiter, Stolp Stettin Osnabrück, DE0988B/C, D4BUB, D4BGB, D4CYB [G]

Dr. Erich Landsmann, Apolda Merseburg, DE1634B, D4MLD, D4BLL [E], D4MSL [G]

Wolfgang Heilgendorff (-1972), Berlin Potsdam Stolp, DE0988B(C), D4BV, D4WVB, D3DJC [G][E]

Max Feder, Cottbus, DE1418 [E], [in G:DE1458?][E][G]

Kurt Lüdke ( $~ 1993)$, Stettin Berlin-Schöneberg, [in G: DE1418B?], D4HWB, D4HLB ?, DL7CO [E]

EK4DZ, Gerhard Thomas

Dr. Hans Littmann, Jena Jueterbog Hamburg Oberkochen, DE0323, EK4BL, EK4FN(1), EK4XN, D4FXB,

D4FNX, D4CJJ [G]

EK4XB(1), Manfred Bayer

Josef Brey, Fürstenfelde Berlin Staaken, DE0017, EK4DDC, EK4KZA, EK4AK, D4BYF, D4KZA, D4AGN [C][V][E]

[CQ-MB 7/27][G], comments on D4RH qso in "hwsat?" [CQ-MB 3/30, 4/30], activity reported [CQ-MB 11/30],

WAC diploma 1930 [CQ-MB 2/34], crystal controlled [QST 6/29 p.67], worked W8ADM (EK4CL) [QST 4/29 p.76],

published book reviews in the military journal "Die F-Flagge" [CQ-MB 1/37 p. 20]

Kurt Lange, Eberswalde Flensburg, DE1231, D4NZB [E] cand. med. dent., D4BTC, D4DHC [CB1937], [E], [G],

same with DL6PU [E][CB1953] Flensburg Einfeld (Neumünster)? Different from DE2424J, D4CSJ [CB1935] Luebeck [E]

Willi Wernicke, Berlin Heiligensee, DE0388F, D4RAC, D4BAC, D4XPF [1936, E], D4FAF [CQ-MB 7/27][G]

[CQ-MB 7/27] ??

Wilhelm Baukisch (-1929), Berlin Charlottenburg, DE0251 [TUC], D4CB [E][C][G], 200W on 20m [QST 4/29 p.76]

Dipl. Ing. Erhard Graff (-1989), Berlin Charlottenburg, DE0473F [TUC], EK4AF(1), EK4BA, EK4CF, EK4WL(2), D4CC, D4RCC, D4CFC, D4CCF, D4BAF, Author in [DASD, 1931], later worked for the Bayerische Rundfunk (Bavarian

Broadcast), died 1989 in München-Ismaning [J:3], photo in [CQ-MB 5/30], author in [CQ-MB 9/30, 8/32],

D4CC, D4BAF heard overseas [CQ-MB 10/30, 7/31]

Günter Zingler, Berlin, DE0844F, D4PDC, D4CDF, D4GCF(1) (near Hamburg [C]), D4CBF, D4GXF, DASD Ausschluss 1938 [G], c.c. station of the month [QST 6/28 p. 68]

B. Eliaschewitsch (-1935), Kowno Berlin, DE0699, RY1E [E], ET1E, XD4LEC [E], studied in Berlin, note "Hwst" about Meteor observation in [CQ-MB 8/30], D4CE elected as WAC member [CQ-MB 8/30][QST 3/1931], died after illness

[LY2YR]

EK4CC, Dipl. Ing. Erhard Graff

Dr. Hanns Kaufmann, Berlin Falkensee Wittenberg, DE0313 [TUC][E], EK4DK, D4CG, [C], D4MGC [G]

Ernst Hoffmann (- 1963), Berlin Stolp Bad Segeberg, DE0890J, EK4AH, EK4BH, D4WHB [G], identical with DJ6DG 
[CB1962]

Buxtehude? [E], author in [CQ-MB 4/32]

EK4CI(1) Fritz Kunze, Berlin, DE0188 [TUC], EK4CM(3) [G]

EK4CI(2) EK4CM(2), Serge Kaplan

EK4CJ

William Fred E. Droese, u2BDQ, Berlin, visited for a time [QST 5/28 p. 60], 954 Summit Ave, Jersey City, NJ [CB 1927]

EK4CK [E]

Schi', near Nauen, near Berlin, D4CK [C][CQ-MB 7/27] ??

EK4CL [E]

Dr. Curt Lamm (1906-1984), DE0152, D4AFA, W8AJX?, W8ADM [QST 4/29 p.76], OZ2CL in 1933, A organic chemist, left Germany for Denmark in 1932. He played an important role in building the DASD. Went 1928 as DASD envoyee to visit ARRL officials, like ARRL secretary K. B. Warner and Col. Foster, W6HM [E][CQ-MB 7/27][OZ7DX][DJ4ZC], author in[CQ-MB 7/30, 7/31]

EK4CM(1) Gerhard Ostermeyer, Berlin Spandau, DE0414F, K4CM, D4CMC, D4FMF, D3DCF [U][G]

EK4CM(2) [E] Serge Kaplan (-1995), Berlin, DE0174, EK4CI(2), EK4IC(2), K4SA, K2SK, K2CL?, D4WIC, D4WMC, PA0CM, DJ0MV. Worked at the Philips Laboratories Eindhoven since 1933, PA0CM [CB1938], then deported to KZ Auschwitz, liberated at KZ Buchenwald, https://www.youtube.com/watch? $\mathrm{v}=\mathrm{mNwSguP7xSc}$. Later moved to Überlingen as DJ0MV, [OOTC 2818][G][CQ-MB 7/27][F:26][C] Station Description [CQ-MB 4/32], author in Funk-Bastler, [CQ-MB 2/30, 10/30, 7/31]

$\operatorname{EK} 4 \mathrm{CM}(3) \quad$ EK4CI(1), Fritz Kunze [G]

$\mathrm{EK} 4 \mathrm{CN}$ Heinz Rath, Berlin, DE0192, K2HR [CQ-MB 7/27][G]

EK4CO(1) [E] Walter Fleck (- 1957), Berlin, DE0466F [E], D4DOC(2), D4CIF, DL7BF [C][G]

EK4CO(2) Max Schultheiss (-1998), Nürnberg Eschenheid, DE1365R, D4NOV, D4BGR, D4NTR, DL1YC [E], [G]

EK4CP(1) [E] “Fritz” Rudi Hammer (1911-1993), Berlin, D4CP, D4JPC 1932 [E], D4CAF 1933 [E], D4AFF 1938 [E], D7AA, DA7AA [E], DK7AA [E], DL7AA [E][OOTC2251], D4JPC heard overseas [CQ-MB 1/32], WAC diploma 1933 [CQ-MB 2/34]

EK4CP(2) Herbert Neltschik, Berlin, DE0737F [E], [C], [G][E]

$\operatorname{EK} 4 \mathrm{CQ}(1)$

$\operatorname{EK} 4 \mathrm{CQ}(2)$

EK4CR

EK4CS [E]

EK4CT

EK4CU

EK4CV

EK4CVJ

EK4CW

$\operatorname{EK} 4 \mathrm{CX}(1)$

Walter Scheibner, Berlin Halensee, DE0763F, D4NQC, D4CRF, D4HGF [G]

EK4QU, Rudolf Asbach [G]

Werner Slawyk (-1953), Berlin-Zehlendorf Schlehdornweg 38, DE1156F, EK4WX, D4RRC, D4VXW, D4BUF, DL1XF,

Author in [DASD, 1931][CQ-MB 6/31][G][E], WAC diploma 1932 [CQ-MB 2/34]
Dipl. Ing. Dr. Max Bunge, Berlin, DE0623F [TUC][C], D4SSC, D4CLF [E][G], D4C

11/1931 [Krotkofalewski Polski 12/1931], crystal controlled [QST 6/29 p.67]

Willi Saunat, Berlin, DE0904F [C], D4FTC, D4BSF, D4GPF [G]

EK4KU

Helmut Fischer (-1993), Berlin Oranienburg, Bremen, Hamburg, DE0989C, D4CV, D4CVC, DL3QO [CB 1951][C]

[CQ-MB 7/27] ??

EK4TR, [CQ-MB 7/27]

$\operatorname{EK} 4 \mathrm{CX}(2)$

EK4CY [E]

EK4CZ

EK4DA [E]

EK4DB

EK4DC

EK4DD

EK4DI

EK4DK [E]

EK4DL

EK4DN

EK4DP

Rolf Wigand (-1944), Berlin-Charlottenburg Bismarkstr. 11, DE0065 [E], D4CXF, [CQ-MB 7/27],

Author in [DASD, 1931], disappeared in 1944, author in [CQ-MB 5/30, 9/30, 11/31, 9/32]

EK4VR, Fritz Weingärtner

Dipl. Ing. Kurt Schlupp, Berlin, DE0474F [TUC], EK4WI, D4CY [E], D4SYC, D4GIW, D4CEF [E], D4DAC [C][G] EK4XU [G][CQ-MB 7/27]

Halle [C], heard by W2JD [QST 4/1929 page 84] ??

Fritz Schnepel (-1979), Königsberg Itzehoe, DE0734A, EK4DBD, D4DBD, D4ASA, DL1IH [G]

[CQ-MB 7/27] ??

[qsl from DE0413, TUC] ??

Max Dittmann, Apolda, DE1165A, D4VDI [G]

Dr. Hans Kaufmann, Berlin, Falkensee, DE0313 [TUC], D4BKD, [C][G], south of Berlin [C]

EK4ABI, EK4ME, Max Drechsler, Halle/Saale, DE0049 [TUC, E]

[CQ-MB 7/27] ??

Hans Gerd Rentsch (->1994), Bremen Berlin, DE1487K, D4LRJ, D4LPP, D4BJK(2), D4CVF, D4CVK, DL1WV [G],

D4LRJ heard overseas [CQ-MB 8/31, QST 7/31]

EK4DV

EK4DW

EK4DZ

EK4EB

communicated with CV5BJ, 11/1931 [Krotkofalewski Polski 12/1931] ??

Karl Doppelhofer (-1958), Graz, DE1388W, DE3801, D4DOW, UO5DK, OE6DK [G]

Gerhard Thomas (-1976), Berlin Neukölln Bad Altheide Hildesheim Fürth Benin bei Glatz, DE1123F, EK4BW, EK4GY, EK4WP, D4HOV, D4BBW, D4OYG, D4BCG, D4HOG, DL7BT [G][E]

EK4EC

EK4EG

EK4EHP

EK4EJ

EK4EK

EK4EN

$\operatorname{EK} 4 \mathrm{EO}(1)$

Friedrich Dahlke (-1943), Liegnitz, Dresden, Fischhausen, DE0379, EK4EG, EK4ADU, D4FGE [E], D4BIA, D4VAA

$[\mathrm{G}][\mathrm{OP}]$

Günter Farchs (Fahros [CB1936:D4CQA]), Königsberg, DE1445A, D4FCE, D4BFA, D4CQA [G][OP]

EK4EB, EK4ADU, Friedrich Dahlke

[CQ-MB 7/27] ??

Horst Melzer, Berlin/Tempelhof Mittweida Königsberg, DE1206F/A, D4DJE, D4FUE, D4BEA, D4CRA [G]

EK4ES, Hans Wottrich

Lothar Balzer (- 1992), Fischhausen Holzminden, DE1337A [TUC], D4ONE, D4KOE, D4BMA, DJ3LE [E],[G][OP]

$\operatorname{EK} 4 \mathrm{EO}(2)$

Dr. Ernst Tomuschat, Stettin Peine Berlin, DE1336J, D4FOE [E], D4BVK, D4JVB [E], D4NTQ [G]

Hans Ruffler (Rüffler), Königsberg Heidelberg, DE1766A, D4FOE [E], D4IHO, D4BRA, D4THA [G][OP] 
EK4EO(3)

EK4ES

EK4EU

EK4EUV

EK4EV

EK4EW(1) [E]

EK4EW(2) [E]

EK4EX

EK4EY [E]

EK4FA

EK4FF

EK4FG [E]

EK4FH

EK4FI [E]

EK4FJ

EK4FK [E]

EK4FM

EK4FN

EK4FO

EK4FP

EK4FR [E]

EK4FS

EK4FT(1)

EK4FT(2)

EK4FU [E]

EK4FV(1) [E]

$\mathrm{EK} 4 \mathrm{FV}(2)$ [E]

EK4FW [E]

EK4FX

EK4KOE

Hans Wottrich (-1968), Königsberg Vorstadt Alfeld, DE0917A, EK4EK, EK4EW, D4PWE, D4FWE, D4ESE, D4BCA, D4DBW, D4DBA [KFL1944], DJ3WP [CB1965][G][E][OP], D4FWE heard overseas [CQ-MB 7/31]

Hugo Fagien, EK4DBA [OP]

M. Mäschke (1908-), Dresden, Tzschimmerstr. 20, 1924 [E][CQ-MB 7/27]

EK4HI, Helmut Oltzscher

Wolf Gutmann (-1991), Königsberg, DE1444A, D4PWE, D4OWE, D4BHA, DL3WG [G][E][OP]

EK4ES, Hans Wottrich, Königsberg, Pr., DE0917A, D4EW [C]

Karl Buchmann, Schaffhausen, DE0207O, [G] probably typo, call is DK4EX in [G]?

Dr. Herbert Schulz (-1984), Königsberg, DE0345, EK4AC(1), EK4FS, EK4VS(2), D4EY, D4FYE [E], D4BGA, D4CSA

[E], DM2ABC [CB1956,1957], DM2ACA [CB1959], DL1OV Rotenburg, 1st qso: Sept 1924 [F:37][C][E][HH]

Bernhard (Benno) Schosinksy (-1985), Hamburg Warnemünde, DE0175, EK4SA(1), EK4TB, D4BAF, D4CKJ [E],

DL1ZU [E], Leitfunkstelle Berlin [G], left DASD in 1929 [G]

Jochim Schneekloth, Hamburg, DE0322 [TUC],[G]

Hamburg [C] ??

Richard Hinz (-1956), Hamburg Braunschweig, DE0960D, D4BHF, D4BBX, D4CNJ, D3JGD, DL1SU [G][HH]

Adolf Zelck (-1959), Stade, DE0105 [TUC], Inspektor, D4NFI, D4BQJ, D4KTJ, DJ4UH [E][G]

Heinrich Rühsen (-1982), Lohbrügge-Bergedorf, DE1118, D4OJF, D4BRJ(1), D4KUJ, DA4LO, DL1RX [U][G][E]

Robert Koch, Sülfeld near Hamburg, DE0876L [TUC], D4CBJ, [G][E]

Friedrich Mildner, Hamburg, DE1073, D4GMF, D4BTJ, D4KVJ [G][HH]

EK4BX(1), Dr. Hans Littmann, Jueterbog Hamburg, DE0323, EK4BL, D4FN, D4XN, D4FNX, D4CJJ [G][C][HH]

Gustav A. Pohl, Hamburg, DE0265, D4OOF, D4BUJ, D4KWJ [G]

Robert Meyer, Harburg Wilhelmsburg, DE0398J [TUM][C][E], EK4HR?, D4FP, D4EPF, D4BGJ, D4KHJ [G][HH], author in [CQ-MB 1/30, 2/30, 4/30]

Rudolf Rapcke (1894-1973), Hamburg, DE0356J [C], EK4HR?, D4FR, D4RRF, D4BWJ, DL1WA,

author in [DASD, 1931][CQ-MB 8/31, 8/32][C]

EK4EY, Dr. Herbert Schulz (-1984) [HH]

Karl Trost, Hamburg, DE0808J, D4ITF, D4CAJ [G][HH]

EK4TV(1), Wilhelm Sattler [G]

Ernst Kammeyer (-1970), Hamburg, DE1235, EK4HL(2), D4CUF, D4KRJ [E], D4BOJ, Op at D4AEO [G][CQ-MB 7/27]

(Braunschweig,[C])[HH][E]

Paul Lescow, Hamburg Volksdorf, DE0320, D4RVF, D4CHJ, D4JIJ [C][HH]

Georg Graba (- 1971), Wesselburen, DE1694V, D4BAV, DL3ZN [G]

Eitel-Friedrich Eddelbuettel (1911-2004), Hamburg Harburg, DE0887J, D4FW, D4IWF, D4CVT in 1932, D4IWF heard overseas [CQ-MB 11/31], D4BSJ from 1933 until 1935/36, D4AJJ [G][HH], WAC diploma 1930 [CQ-MB 2/34]

EK4GA(1)[K,75] Erich (Hans) Körber, Liegnitz, DE0642U, D4GAG 1932 - Eichwellendienst [CQ-MB 3/32][V][G][K], activity reported [CQ-MB 11/30]

EK4GA(2)

EK4GE, Walter Jäckel

$\mathrm{EK} 4 \mathrm{~GB}(1)$

EK4GB(2) Herbert Alfke (- 1993), Stolp Frankfurt/Oder Berlin, DE0986G,
DA2OA, DL1VR [G], D4GGB heard overseas [CQ-MB 10/31]

Schönbrunn, Wilhelmshaven, Graudenzerstr. 22 [CB 1951][E], D4NRG heard overseas [CQ-MB 3/31, 6/31]

EK4GC Herbert Schastock (-1985), Züschen Beuthen Fritzlar Frankfurt Imperia, DE1139G, D4HAG [E], DL1AO (1952), DL1AZ (after 1958), YA1AO [E], I1UNO [G]

EK4GD(1) Günter Heimlich (-1987), Reichenbach/Schl., DE1145G [E], D4RDG, D4CAK [U][G]

EK4GD(2) [E] Jochen Gumpel, Dessau 1926 [F:24][DL7AY letter]

EK4GE

Walter Jäckel (-1936), Breslau, DE1185, EK4GA, D4WEG, D4WAG, D4HNG, D4BBG [G]

EK4GG

Kurt Jana (->1963), Königsluther Kreuzburg/Oberschl., DE0894G, D4WWG, D4BKG [E], D4HXG, DL1VG 1963, $[\mathrm{G}][\mathrm{E}]$

EK4GI

EK4GJ

EK4GK

EK4GL

Herbert Stoffregen (-1963), Gevelsberg, DE1140G, D4GIG, DJ3UM [G], D4GIG Eichwellendienst [CQ-MB 3/32]

Otto Selck, Beuthen Hildesheim, DE0793G, D4GJG - Eichwellendienst [CQ-MB 2/30, 3/32], EK4ZN [G]

Joachim Siekmann (-1966), Karlsruhe Weidenburg Wetter/Ruhr Köthen Bad Salzbrunn, DE1178, D4MKG, D4GKO, D4OPG(2), D4WXG, D4WXD [E], D4BYG, DL1BR [E], [G][E], 80m activity reported [CQ-MB 8/30]

Günther Przyrempel (-1935), Hartenberg, DE1132G, D4KLG, D4HVG, D4BIG, D4JRG, see EK4GC [G], activity reported [CQ-MB 11/30], obituary in [CQ-MB 11/1935]

EK4GM

EK4GN

EK4GO [E]

EK4GP [E]

Fritz Boehmer, Frankfurt/Oder Breslau Rottenburg/Laaber, DE1181, D4KMG, D4QPG, D4BAG, D4AGG [G]

Theo Spieske (- 1990), Breslau Beuthen, DE0715G, D4CGM, D4BTG, [G], likely identical with DL9RZ Frankfurt $[\mathrm{CB}][\mathrm{E}]$

Adolph (Alfred "Alf") Dickfeld, Berlin, Breslau, DE0864F, D4GO, D4BQG, D4FEF, D4PEF [G][C], author in [CQ-MB 10/30, 1/31, 6/31], heard overseas [CQ-MB 11/30, 2/31]

Dr. Karl Wirtz (1910-1994), Breslau Halle Köln Göttingen Karlsruhe, DE0939, D4OPG(1), D4BRG [G][C] Nuclear Physicist, member NSLB, held at Farm Hall in 1945 [W], D4OPG signals heard overseas [CQ-MB 11/30, 3/31], author in [CQ-MB 2/30, 7/30]

EK4GQ D4GQ near Breslau [E][V]?? 
Erich Wagner (1911-1991), Reichenbach/Schi Varel Würzburg, DE0915G, EK4SAR, EK4SAG, D4CBG, DA0BA?, DA5BA, DL1LD [G][V][E]

EK4GS [E]

EK4GT

EK4GU

EK4GV

EK4GW [E]

EK4GX

EK4GY

EK4HA

EK4HC [E]

EK4HF [E]

EK4HG

EK4HH(0)

EK4HH(1)

EK4HH(2)

EK4HH(3)

EK4HH(4)

EK4HI [E]

EK4HJ(1)

EK4HJ(2)

EK4HK

EK4HL(1) [E]

EK4HL(2)

EK4HN [E]

EK4HP

EK4HR

EK4HT

EK4HU

EK4HV [E]

EK4HW(1)

EK4HW(2)

EK4HX [E]

EK4HY(1) [E]

EK4HY(2)

EK4HZ

EK4IA [E]

EK4IB [E]

EK4IC(1) [E]

EK4IC(2) [E]

EK4ID

EK4IG

EK4II
Erich Hensel, Festenberg Waldorf, Saalmünster, n. Breslau, DE0421G [E], D4LGS, D4RXB [G]

Kurt Haeske, Breslau Göttingen Würzburg Rittmarshausen, DE0638G [TUC][C], D4TTG, D4BPG, D4IPG [G]

Joachim Froböß (-1945), Breslau Köthen Gispersleben, DE0911G [TUC], EK4HT, D4MSG, D4BHG [E], D4BTO,

D4AFF?, D4HTG [E][G][V], disappeared

Paul Strelczyk (-1983), Opperau Breslau Krumbach Berlin, DE0762G [TUC][C], D4WVG, D4BEG, D4GEG, D4HQG, DJ7AV [G], Op: at EK4ABV

Erich Rachner (1904-1984), Breslau Stockdorf Maplewood NJ Martinsmoos Schömberg, DE0639, D4PGW, DL1AV

[CB1949], DL2WE [E][CB1969-1983], [E][C][G] 1954: Erich Rachner, 67 Montgomery Ave, Irvington 11, NJ

[Cornell-Dubilier 5/1954], died in NJ? [ancestry.com], activity reported [CQ-MB 11/30], author in [CQ-MB 10/31],

photo in [CQ-MB 5/30]

Dr. Erich Wiedemann, Breslau, DE0327, EK4TZ, D4BXG, D4EXG [G]

EK4DZ, Gerhard Thomas

[CQ-MB 7/27] ??

Dr. med. Hans Berner, Chemnitz, Sachsen, DE0489U [TUC], EK4HV, D4RVM, D4BIL [E], D4MPL [CB1936], D4MPT $[\mathrm{CB} 1938][\mathrm{U}][\mathrm{G}]$

Leipzig [C] ??

Friedrich Bock (-1945), Leipzig Holzhausen, DE1060M, D4LGH [E], D4LQH, D4BFU [G][V] died in Yugoslavia, D4LGH heard overseas [CQ-MB 11/30, 5/31][V]

A. Gohlke, Hamburg, DE1367F [TUC], D4HH, D4BSO [G]

Ango Taig, Berlin, DE1471F [E], D4HH, D4PSO [E], D4USO, XR1AA [CQ-MB 10/1936][G],

Chinese student at the Technische Hochschule Berlin [QST 7/34]

Kurt Hartenstein, Plauen München, DE0082 [TUC], EK4LM, EK4UU, D4AAP, D4GML, D4SHH, D4BUU [G]

Referatsleiter MfS HA I/2 1956-1959, responsible for nachrichtendienstliche Arbeit gegen das Auswärtige Amt

[MFS, 2008]

Otmar Pregler (1913-2009), Bamberg, DE1494R, D4BSO, Oberstudiendirektor [G][Obit franken.de]

D4HH, Leipzig, 1930 [V] ??

Helmut Oltzscher (-1958), Plauen Dresden Kiel Höxter, DE0930 [C], D4LRM, EK4MR, D4HIX, EK4EV,

D4BAM(1) [G]?, D4ELF, D4CJM, D4BQU, DL3DZ [G][E], D4LRM signal received overseas [CQ-MB 3/31, 1/32]

Max Jung, Finkenkrug, DE0695C, EK4NJ, D4OKC, D4BJK(1), D4BHT, D4OKT [G]

Phillip Grode (1893-), Leipzig, DE0952U, D4GJH [E], D4BBU, D4PAU [G], airplane engineer, entrepreneur, bio in

[CQ-MB 7/1937]

Christoph Schmelzer (1908-2001), DE0078 [TUC], D4LKH, D4BIU, Jena Fraunhoferstr.1, D4biu, [F:28], Forschung GSI Darmstadt [G][W]

Kurt Illing (-1946), Leipzig, DE0195U [E][TUC], D4HL, D4MLH, D4RPM, D4AAU, D4BAU, D4AUU [C][G][V],

photo in [CQ-MB 5/30], author in [CQ-MB 12/30], D4RPM heard on 10 meters [Journal des 8 7/1933]

EK4FU, Ernst Kammeyer, Hamburg (suffix freely invented), DE1235, K4HL, Op of Ki6, EK4AEO [J:11][D]

Rudolf Riedel (->1994), Plauen, Vogtland, DE0827U [C], EK4HW(2), D4LNH, D4WUM(1), D4BUM, DA4HN, DA5XX,

DL3DW Herford [C][E]

?? [QSL from EK4VJ, E]

EK4FR?, EK4FP? [HH][G]

EK4GU, Joachim Froböß

Herbert Queck, Berlin Dresden, DE0757F, D4LUH, D4BAM(2) [E], D4AWM, D4YTM? [G][C], D4LUH

heard overseas [CQ-MB 6/31][DG6WAN]

EK4HC, Plauen, Vogtland, D4HV [C][G]

Albin (Willy) Schädel, Leipzig, DE0205, D4MWH, D3BTU [G]

EK4HN, Rudolf Riedel

Dr. Rolf Lentzsch (-1983), Dresden, DE0682M, D4HX, D4LXH?? [E], D4NXH, D4BHU, D4BSM [E], D4PFM [C], DJ2IW, author in [QST 9/28 p. 68; CQ-MB 6/31]

Herbert John, Leipzig, Kielerstr. 67, Author [CQ-MB 11/34][E]

Dipl. Ing. Heinz Funck (- 1956), Sebnitz Hildesheim, DE1267M, DE5431, D4LYH, D4BZM, D4LQM, DL1VB [E], member FWGM [G]

Dr. Gerhard Jahn (->1994), Rudolstadt Jena Berlin München, DE1350L/F [TUC], EK4AI(2), D4INI, D4VNI [E], D4IND, D4KAI, D4BML [E], DL7BJ [G], heard in England [QST 8/1929]

EK4IC(1), near Saalfeld, Thüringen, [E]

Fritz Löscher, Berlin Weida near Gera, DE0794F, EK4WO, D4MOW, D4MOF, D4LBT, D4BOL, D4MUF [G][E]

Dr. Otto Gerhard Baez (-1982), Berlin Apolda, DE0725, EK4IA(2), EK4EO, EK4TQ, D4IC [G], D4KOE, D4NAI, D4BBL, D4MLL [CB1936], D4MLF [CB1939], DL7AB [G][E], D4NAI signals heard overseas [CQ-MB 3/31]. In 1951: Laboratorium für Elektromedizin und Radiotechnik -other key personnel: Helmut Schoettle. Kaiserstr. 22,

Berlin-Lichterfelde Ost [CIA report 1951]

EK4CI(1), Serge Kaplan

EK4LH, Dr. Werner Dickertmann, Hagen, DE0366, D4NDI, D4BCI [G][CQ-MB 7/27]

EK4RD

QSL from ON4EL to D4II 1930 [E] ?? 
Karl Siegert, Kötzschenbroda Radebeul, DE1271M, D4KOI, D4BWM [E], D4LNM [G][E]

EK4IM(1)

EK4IM(2)

EK4IP [E]

EK4IS

EK4JA

EK4JB

EK4JK

EK4JL(1)

\section{EK4JL(2) [E]}

EK4JM

EK4JO

EK4JP

EK4JS

EK4JU

EK4KA [E]

EK4KB

EK4KC

EK4KD

EK4KG(1) [E]

EK4KG(2)

EK4KH

EK4KK

EK4KKÜ

EK4KL

EK4KN

EK4KO

EK4KP

EK4KQ

EK4KS

EK4KU(1) [E]

EK4KU(2)

EK4KV [E]

EK4KX

EK4LA

EK4LB

EK4LC

EK4LD(1)

EK4LD(2) [E]

EK4LF
Kurt (Bruno) Braune (1901-1978), Dresden Eisenach Stuttgart Kiel, DE0784M, D4DLI, D4CIM, D4YTM, D4BPB, DA1AG, DL1CN, [G] Dresden-Oberloschwitz, Osaerstr. 1, [C], DL1CN Curt Braune, Kiel, Hedenholz 8, [RL1977] Oskar Lieberum, Erfurt, DE1300L [TUC], D4BMI(1), D4BQL, D4WHL [CB1938], [G]

Dipl. Ing. Wilhelm Fischer, Hamburg Weida Gera, DE1428M [E], D4IMI, D4BHL [G]

Helmut(h) Wolf (-1985), Jena Berlin Halle Marburg, DE0878L, D4RPI, D4BNL, D4MTL, DC7UV [G], author in [CQ-MB 3/31, 2/32, 6/32]

Dr. Karl Stoye, Berlin Quedlinburg, DE0035D, D4FSI(1), D4BED [E], D4FID [G], author in [CQ-MB 4/30, 2/31,..]

Dr. Georg Mirus (-2017), Wertheim Berlin, DE1214F, D4RJA, DL1MM [G][CQWA18304]

Hugo Horwitz (-1983), Bünde, DE0255, Caseros, Argentina, LU7DH [CB1962-1988][G][Tablada Cemetery,

Buenos Aires, hebrewsurnames.com]

Erwin Klein (1901->1994), Osnabrück, DE0052 [TUC], D4LKJ, DA5KD, DL1PS Erwin Klein, Osnabrück,

Lieneschweg 98 [CB1951][F:26][G][D]

Hans Prost, Osnabrück, DE0058 [TUC][E], K4JL, D4LLJ, D4BOK [CB1934], D4JUK [CB1936],

D4FAF [CB1938] [F:26][C][D][CQ-MB 7/27], signals heard overseas [CQ-MB 10/30, 7/31],

WAC diploma 1930 [CQ-MB 2/34]

K4JL "Jolly" near Hannover [C] ??

Wilhelm Brockmann, Lengerich, DE0020, D4JM, D4FMJ [G]

Albrecht Leyn (->1951), Hannover, DE0935, EK4PL, EK4QE, D4POJ, D4BBK, D4JMK, D4POL [G], worked in 1942 for Telefunken at Bernhard Navigation system [Bernhard], WAC diploma 1933 [CQ-MB 2/34]

Dr. Ing. Klaus Prost, Osnabrück, Berlin München, DE0617K, EK4VK, EK4VL, D4KPJ, D4BTK [E], D4JUK, D4JUF [G]

August Schritt, Osnabrück, DE0146, D4FSJ, D4BYK, D3BOK [G]+

EK4RY, Oskar Becker, Köln Riehl, DE 0654, D4JU, D4JUR, EK4RY, DL1JU [E], [G]

Dr. Georg Weiss (-1980), DE0327, Kassel, [C][CQ-MB 7/27], EK4SM(2), EK4WX, DF3FS [G]

Gustav Bock, Frankfurt, DE0178, EK4ABQ, D4OJT, D4BFT [G]

Hans Clermont, Köln Braunschweig Bad Homburg, DE1252I, EK4LI, EK4BI?, D4CCK, D4CJT, D4BJI, D4OXT [G]+

Dr. Werner Schuster, Ulm Bad Neuheim Petersdorf i. R., DE1151T, D4FDK, D4FDX, D4BRT, D4OTT [G],

D4BRX [E] Dr. Schuster a/B Motorschiff “Cumberland” on east asia trip, Hamburg-Amerika Linie [CB1934]

Dr. med. Walter Lampe, Göttingen Eschwege, DE0626T [C], EK4KN, EK4KX?, D4LNK, D4BOT [E], D4ORT,

near Kassel [C][G]

EK4AB, EK4ABR, Ferdinand Bödigheimer, Frankfurt, DE0069 [G]

Kurt Mania (-1987), Köthen Kiel Bredeneek, DE1623V, D4NEF, D4BDV, D4JEV [E], D4JED, DA6KH [E],

DL1JG, OE2KMN [G], started 1929 [CQ-WA 2-1983], son is DH2LAB

Ernst Kuhn, Wiesbaden Friedberg, DE0775T, D4NKK, D4BBT, D4OFT, identical with Ernst Kühn (-1988), Friedberg, DJ2QZ [E], F0QZ [G]

[CQ-MB 7/27] ??

Franz Noether (-1931), DE0038, D4ELK, op at KQ5, D4ABN, Kaufmann, Condor-Syndikat Brasilien, died 1931 in plane Crash (Natal) [OV F12 website][Van der Mey, 2012], obituary in [CQ-MB 10/31][D]

EK4KG, Dr. med. Walter Lampe

EK4ON, Adolf Kinzinger

Fritz Kühne (1910-1982), Leipzig, München Garmisch Friedrichshafen, DE0226, D4RKP [E], D4ONT, DL6KS [G]

Otto Hunstock (-1961), Demmin Berlin Hamburg Stade, DE1636L, D4WAF, D4BNJ [E], D4KQF (1938), DA5PA,

DL1YU (died in Brasil) [G]

Dr. Hubert Seeanner, Friedberg, DE0899T, D4DSK, D4BPT, D4OST [G], [Patent, 1937]

Ernst Reiffen (-1952), Cassel, DE0057K/F, K2OO?, K2DO [E], EK4RU, EK4KV, D4RUK, D4MUC, D4CFF, D4BVR, D4KCL, D4BVT 1939 DL1HI, operated Kdzl as a student in Danzig [C][G], author in [QST 11/28 p.56, 10/28 p.70; CQ-MB 4/31], WAC diploma 1930 [CQ-MB 2/34][QSL EK4KU ex K2DO 1927 E]

Helmut Baumert (1914-1991), Fürstenwalde Spree Wilhelmshaven, Breisach/Rhein, DE1611C, D4DTC [E], D4FIB, D4BOC [E], D4YYA, DL1QT [G], chemist, Waffen SS war reporter with Standarte Kurt Eggers, later worked for BND under pseudonym "Bachmann" [Lecorte, 2015]

Ernst Reiffen (-1952), EK4KU(1), Bewegliche Station (portable station), bei Kassel im Wald [E]

? EK4KG, Dr. med. Walter Lampe, Kassel, spaeter Eschwege [OV F12 website]

Adolf Dreesbach (-1974), Köln Dellbrück, DE1130, EK4LN, D4LAL, D4BII, D4IVI, DL1WK [G][CQ-MB 7/27], heard by PL423 [Krotkofalewski Polski 6/1933]

Hans Otto Koch (-2012), Köln/Lindenthal Oberlahnstein, DE1253I, EK4LK, D4SKL [E], D4ITI, D4BGI,

DL6HK [CB1972 and later] [G] D4SKL heard in England [QST 8/1929]

Erich Kaminski (1910-1944 kia), Wanne Eickel, DE1082H [TUC, E], D4MKL [G][QRZ]

Club KL4, EK4ABI or Max Dressler, EK4ME, EK4DL, Halle/S., used 10/1925-2/1926 [DE0195 qs1, TUC]

Dr. iur. Harald Dickertmann (1909-1994), Hagen Aachen Übersee (Chiemgau), DE0362, Kan2, D4NDL, D4IFH, D4IFF(2)

Berlin, D4BDH Hagen (D4BCH), D4IFG Dr. H. Dickertmann, Ratibor, [C][CQ-MB 7/27], DL7EG [E], [G], author in [CQ-MB 6/32], see 1933 Letter to exit the DASD [K:103], Waffen-SS, worked after WW2 as a judge (Bundesrichter) in Aachen, retired to Bavaria, biography in [W]

Richard Fischer (->1994), Münster Frankfurt/aM Duisburg, DE1259H [E], EK4RF(2), D4SSV, D4CEL, D4BFH, D4IHH, D4IHT, (D4CUL, D4BVH ?), DL3CW [G] 
EK4LG

EK4LH

EK4LI

EK4LJ

EK4LK(1)

EK4LK(2)

EK4LL

EK4LLD

EK4LM

EK4LN

EK4LO

EK4LP(1)

EK4LP(2)

EK4LP(3)

EK4LQ

EK4LR

EK4LS(1) [E]

EK4LS(2)

EK4LV

EK4LX

EK4LY

EK4LZ

EK4MA [E]

EK4MB [E]

EK4MC [E]

EK4MCA

EK4MD

EK4ME

EK4MF

EK4MG [E]

EK4MH

EK4MI

EK4MJ

EK4MK

EK4ML [E]

EK4MM

EK4MO

EK4MP [E]

EK4MPA

EK4MQ

EK4MR

EK4MT(1)

EK4MT(2)

EK4MW

EK4MX

EK4MZ
EK4DFK [G]

Dr. Werner Dickertmann (1908-1989), Hagen, DE0366, EK4ID, D4NHL, D4NDI, D4BCI, D4IEH, D4BCH, D4FKD

[G], see 1933 letter to exit the DASD [K:103], lawyer after WW2 (Rechtsanwalt und Notar), brother of Harald

Dickertmann, EK4LD, see HD's entry in [W]

EK4KC, Hans Clermont

Ing. Günther August Heinrich Engels, Solingen Hamburg, DE0342, D4PJL, D4BIH, D4IHK, D4MVF [G]

EK4LB, Hans Otto Koch

EK4LC, Erich Kaminski(y)

EK4PT, Ernst Tiefenbach

[CQ-MB 7/27] ??

EK4HH(2), Kurt Hartenstein, Plauen, DE0082 [TUC], D4GML, EK4UU, EK4AAP [G]

EK4LA, Adolf Dreesbach

EK4RK, Rudolf Helbig

Max Petzold (-1930), Dortmund, DE0301 [TUC][C], D4DPL, D4SZI [G], obituary [CQ-MB 7/30]

Hinrich Kruse, Hamburg, DE0304 [G]

EK4AU, Rudolf Romeike

Dr. Erich Schneider, Essen, DE0938, D4RQL, D4BEH, D4IGH [G]

Paul Bonert (-1937), Barmen, DE0914, DE0977H, D4NRL, D4BMH [CB1936], D4BWH(2)? [G]

Max Petzold (-1930), Dortmund, DE0301 [TUC][C][CQ-MB 7/27][G]

EK4AU, Rudolf Romeike

Prof. Dr. Werner Nestel (1904-1974), Stuttgart Berlin Hannover Ulm, DE0090, D4LV, DL1ZE, Joined Philips-Radio Berlin in 1928 and the Telefunken GmbH in 1937. Instrumental in the introduction of VHF broadcast (he installed with Lothar Rhode, DJ5LR, first VHF transmitter in 1949), and 625-line TV broadcast. Director of the NWDR (Northwest German Broadcasting) 1947-1955 [Spiegel 6/7/1950], head of R\&D Telefunken in 1956 [W]

[heard Wireless World 12/1925]

Gustav Eduard Vach (-1982), Bremen Schildeck, DE1251A, D4VXL, D4VUL, D4BVA, D4BXH, D4CVA, DL1NU [G]

Otto Knüppel (-1969), Barmen Oberhausen, DE0756H [TUC], D4SYL, D4BJH, DL1OH, Op. at EK4ACJ [G]

EK4RW, Dr. Otto Wilke

Werner Pilz (-1976), Syrau Plauen-Vogtl. Neuenkirchen Rheine, DE0748, EK4BP(1), D4MPB [E] claimed in [U][G] but writing looks different, probably D4BAM(1) [E] - same layout and writing, D4BFM, D4RPH, D4DGU, DL3AY [G][E] Dr. Horst Körber, Dresden, DE0361U, D4MB, D4LBM [C], “detained by police” on 1929 QSL [E]

Dresden [C], friend of D4MB [E] ??

EK4DBA, Hugo Fagien (-1953), Königsberg, KPL, DE0673, D4SDA [E][CQ-MB 7/27]

Carl Otto Grämer, Plauen Chemnitz Zwickau, DE1221U, D4WDM [E], D4BNM, D4BNU, D4PKU [E] license revoked in 1939 [CQ-MB4/1939], [G][DM6WAN], author in [Grämer, 1953]

Max Drechsler, Halle, DE0049 [TUC], after 1931, EK4DL, EK4ABI, D4ABI, D4DEM, D4BAL, D4ALL, D4PXL [CB1936], D4ALU [RL1938], photo in [CQ-MB 5/30][G][D]

Dipl. Ing. Herbert Petzold, Plauen, Wartburgstr. 2, DE0947, D4MFM [E], D4PMM, D4BKU [E], D4PHU, D4PHM [E], D4MFM signal received overseas [CQ-MB 7/31, 1/32], WAC diploma 1931 [CQ-MB 2/34], heard by PL198, 11/1931

[Krotkofalewski Polski 12/1931]

Martin Schurig (- 1994), Fuchshain Leipzig Land, DE1119U, D4EGM [E], D4BDU, D4PCU [E], D4BDS, DM2AHM, Y21KM 1st qso $1929[\mathrm{G}][\mathrm{E}]$

Eduard Ruhig, Halle München, DE1469P/L [TUC], D4UHM, D4UMH, D4FML, D4BGL [G],

D4UHM signals heard overseas [CQ-MB 7/31]

Oswald Kruschwitz, Gr. Ottersleben, Möser, Bitterfeld, DE0985D, D4RIM, EK4BD, D4GCD, D4LNB, D4BEL [G]

EK4MJ, Kurt Zschoche

Kurt Zschoche, Dresden, DE1066, EK4MJ, D4NKM [E], D4KZM, D4BXU, D4LPM [G]

D4NKM heard overseas [CQ-MB 7/31], heard by PL297, 11/1931 [Krotkofalewski Polski 12/1931]

Dipl. Ing. Heinz Ifland (-1956), Leipzig Berlin, DE1122 [E], D4RML, D4BCU, D4GKF, DL7BI [G][CQ-MB 7/27]

Erich Nitsche, Bautzen Berlin, DE0774C [E], EK4BM(2), D4JMM, D4BGH(1), D4LEM, D4BGM, D4LFM, D4LFF [G]

Rudolf Bolick, Wittenberg Bergwitz/Halle, DE1154L/U, D4MOM, D4BCL [CB 1934], D4MML, D4MMU [1938, E], D4MOD, D4BHH(2)?, D4BXL [G][CQ-MB 7/27], qso with YIKR on 9/1930 [CQ-MB 11/1935], Erna Bolick, DE3600 [E] ? Mayer, near Leipzig, EAMP, UOMP, Wien XVIII Martinstr. 71 [C: old EA-MP from Austria][OE1HWC]

[CQ-MB 7/27] ??

qso with W2CJJ on 8/1931 [CQ-MB 11/1935] ??

EK4HI, Helmut Oltzscher, Plauen,

Horst Bötel, Leipzig, DE1137U, D4KTM, D4KSM, D4BWU, D3BUU [G]

EK4MW, Friedrich Wirth

Friedrich Wirth (-1990), Wittgensdorf Leinzell, DE1153U, EK4MT, D4RWM, D4LQH [E], D4BMU, D4BMM, D4PJU [E], DL8OB [E], [G][DM6WAN][CQ-MB 7/27]

Werner Müller (->1995), Bauzen, DE0764U [TUC], D4LEM, D4LEU, D4BDM, DK8MX, DM2ACM, Y21CM, DL1LWM [G]

Heinz Breitfeld (1912->1977), Chemnitz Berlin Stollberg/Sachsen, DE1351F/U [TUC], D4RZM [E], D4WPH, D4BJU [E], D4BEM, D4KGF, D4PGP, [G] signals heard overseas [CQ-MB 7/31], Breitfeld worked in a Telefunken-

affiliated company in Chemnitz since 1934, and at Telefunken in Berlin in the electro accustics department since 1936 
EK4NAC

EK4NAH

EK4NAO

EK4NB [E]

EK4ND(1)

EK4ND(2) [E]

EK4NF(1)

EK4NF(2)?

EK4NG

EK4NH

EK4NI [E]

EK4NJ(1) [E]

EK4NJ(2)

EK4NK [E]

EK4NL(1)

EK4NL(2) [E]

EK4NN

EK4NTB

EK4NU

EK4NV [E]

EK4NW(1)

EK4NW(2) [E]

EK4NX [E]

EK4NZ

EK4OA(1) [E]

EK4OA(2)

EK4OAO

EK4OB

EK4OC(1)

EK4OC(2)

EK4OD

EK4OF

EK4OG

EK4OJ

EK4OL(1) [E]

EK4OL(2) [E]

EK4OM

EK4ON(1)

EK4ON(2)

EK4OO

EK4OR [E]

EK4OS

EK4OU(1)

EK4OU(2)

EK4OV

EK4OW(1)

EK4OW(2)

EK4OY

EK4PA

EK4PD

EK4PI

EK4PL(1)

Ludwig Schanz, Mainz, DE0656T [C], D4NAN, D4BTD, D4OUT(2)?, D4BDT, D4OHT [G], identical with EK4NA, Darmstadt QSL [E]?, D4NAN heard overseas [CQ-MB 3/31]

[CQ-MB 7/27] ??

[CQ-MB 7/27] ??

[CQ-MB 7/27] ??

Frankfurt am Main, D4NB [C] ??

EK4VD(1), Adolf Weigold

50km near Frankfurt, DE0751 [E]

Dr. Ludwig Fauldrath (-1956), Darmstadt, DE0365T [C], EK4VN, D4BFN, D4BWT, D4NOI, DL1DU [G], author in [CQ-MB 1/32]

Heinz Hertel, Berlin, DE1552F, D4FBW, D4BQF, D4GNF [G]

Friedrich Herwig (- 1967), Koblenz Berlin Wiesbaden, DE0786T/F, EK4OW, D4DGN, D4CET, D4BEQ, D4OWF, D4OWT, DL1XP [E], [G]

Walter Hunsinger, Darmstadt, Frankfurt, DE0447T [TUC][C], D4GHN, D4CIT [G]

near Frankfurt a. Main, D4NI [E], heard by W2JD [QST 4/1929 page 84] ??

Frankfurt a. Main, D4NJ [E] ??

EK4HJ [G]

Theodor Körner, Bad Kreuznach, DE0488T [TUC][C], ex EK4RN, D4NKN, D4BDQ, D4CDK?, D4CDT, D4IXT,

$[\mathrm{C}][\mathrm{G}][\mathrm{E}]$

Willi Laun (->1994), Darmstadt Eberstadt Böblingen Freiburg Roßdorf, DE0964O, D4NZN [E], D4NLN, D4BLT [E], D4OON, D4OOB, D4OOO, DL1DG [G]

Frankfurt am Main, D4NL [C][CQ-MB 7/27] ??

Wilhelm (Willi) Bender, Darmstadt, DE1052 [TUC], D4INN, D4BNT 1933 [E], D4BED?, D4OQT, op at D4BAF [G][CB]

[CQ-MB 7/27] ??

[CQ-MB 7/27] ??

Hermann Woltmann, Darmstadt Strehlen, DE0412O [TUC][C], EK4NW, D4OIP, D4BET,

D4OIT [C][G][CB], author in [CQ-MB 6/30, 2/31, 5/31, 8/31]

EK4NV, Hermann Woltmann [CQ-MB 7/27][G]

$70 \mathrm{~km}$ nördlich Frankfurt [C] ??

Darmstadt [C] ??

Frankfurt a. Main, D4NZ [E], communicated with CV5BJ, 11/1931 [Krotkofalewski Polski 12/1931] ??

EK4TC, Dipl. Ing. Werner Manecke, Karlsruhe, [E][CQ-MB 7/27][G][HH], heard by W2JD [QST 4/1929 page 84]

Hubert Eschert, Mannheim, DE0928, D4WAO(2), D4BMO 1934, D4NMO 1936, D4LYO [G]

[CQ-MB 7/27] ??

Adolf Brender (-1986), Nonnenweier Langenschiltach Schwanau, DE1173, D4BBO, D4BBY, D4BOO, D4SKO(2) 1938,

DL6JP [G][C][CB], communicated with CV5BJ, 11/1931 [Krotkofalewski Polski 12/1931] ??

Franz M. Koch, Manheim (- 1984), DE1053O, D4BLO, D4NLO, DA1DU, D4AEF, DL1LN [CB1951-RL1977]

EK4UI, Luwo Schäfer

Rudolf Mirche (- 1976), Manheim, DE1262O [TUC], D4UDO [E], D4IOO, D4NFO, D4BFO, DL1LP [G][E],

WAC diploma 1933 [CQ-MB 2/34]

Thomas Faul, Heidelberg, DE1502 [TUC], D4FFO, D4BIO, D4NIO [G]

Franz Ströck, Mannheim, DE1488O [TUC], D4SKO(1) 1932 [E], D4BCO(2) 1935, D4NJO [G]

heard by W2JD [QST 4/1929 page 84]??

EK4UA(1), [C] via EK4ABF, [E][CQ-MB 7/27]

EK4PA, Norbert Lechleiter

Wilhelm (F.?) Doering, Karlsruhe, DE0015T, EK4ON, EK4ABF, KI2 [F:26][G]

Adolf Kinzinger, Mannheim, DE1136O, EK4KO, D4ON, D4UNO, D4DIN, D4PKO, D4BDO 1934,

D4NDO 1936 [G][CB]

EK4OM, Wilhelm Doering

Ernst-Hans Reisenauer (- 1975), Wien, DE0906 [C], OE1IU [C][G]

(Coordinates for Mannheim) Baden, D4OR [C] ??

[CQ-MB 7/27] ??

EK4OW(1), Dr. Erich Walter

Max Weinaug (- 1991), Schwerin Hamburg Wallerstein Berlin, DE2212J, D4AVB, D4BJB, OZ7OU ?, DL7GW [G]

EK4ABG, UHU, Otto Anton Klotz

Dr. Erich Walter, Manheim Erlangen, DE1056, EK4OU, D4AWO, D4ZUO, D4BHO, D4NHO [G],

D4AWO heard overseas [CQ-MB 7/31]

Georg Freund (-1971), Berlin Helle Ammerland Waldenburg Bad Zwischenahn, DE1318A/J, D4PWG, D4PGG, DL9HM [E],[G] Address in CB1957: U-1 Lager Barracke, Elmendorf ueber Oldenburg [CQ-MB 7/27] ??

Norbert Lechleiter, Mannheim, DE0923O, EK4OL(2), D4POL, D4BNO, D4NNO [G][CQ-MB 7/27]

EK4PO, Otto Engelke

Wilhelm Ilse (-1980), Göttingen Hettensen, DE0114, D4KIP, D4CDK, D4UUD, DA5ZA, DL1TJ [G]

Cai W. Jensen Op., Bremen, [C] 
EK4PL(2) [E]

EK4PL(3)

EK4PM

EK4PO [E]

EK4PT

EK4PTR

EK4PU

EK4PV

EK4PX(1)

EK4PX(2)

EK4PX(3)

EK4QA [E]

EK4QB [E]

EK4QD [E]

EK4QE [E]

EK4QF [E]

EK4QG

EK4QI

EK4QJ

EK4QK

EK4QL [E]

EK4QM [E]

EK4QMK

EK4QN [E]

EK4QO [E]

EK4QQ

EK4QR [E]

EK4QU(1)

EK4QU(2)

EK4QV(1) [E]

EK4QV(2)

EK4QV(3)

EK4QW(1)

EK4QW(2) [E]

EK4QX

EK4QY(1) [E]

EK4QY(2)

EK4QZ

EK4RA [E]

EK4RB

EK4RC

EK4RD

EK4RE [E]
EK4JO, Albrecht Leyn, Bremen [E][U]

Johannes Andresen (-1950), Flensburg, DE1695V, D4BAV, D4JAV, D4ADT [G],

not identical with Dr. med. Johannes Andresen, DE9159, DL3OK [CB1956-1962][DARC M03 History page]

Willy Bremer, Wenden, DE1111M, D4FMP, mayor of Wenden, baker [DJ3JD][G][CQ-MB 7/27]

Otto Engelken (-1976), Delmenhorst Bentheim, DE1124K, EK4PD, D4LOP [E], D4MOP?, D4NLQ, D4BHK [E], D4JNK,

DJ7OX [G][C][E] 80m activity reported in [CQ-MB 8/30], D4LOP heard overseas [CQ-MB 9/31], D4NLQ communicated

With SP1AH, 11/1931 [Krotkofalewski Polski 12/1931]

Ernst Tiefenbach (-1989), Bremen Braunschweig, DE1110J, EK4LL, D4KTP, D4LOD, D4PLL, D4AFY, D4CHK,

D4KTP, D3COD, DA5JW, DL3BW [G]

[CQ-MB 7/27] ??

Werner Diefenbach (-1974), Durach Kempten Berlin, DE0955F/P, D4RPU, D4BEP, D4MXV, DL3VD [G]

Dr. Reinhard Hafner ( 1990), Vechta Darmstadt, DE0484H [TUC][C], D4PVT, DL1NH [CB1965], F0UI, [G]

Wilhelm Schierenbeck (1889-1957), Bremen, DE0705, D4UXP, D4PKQ, D4BKQ [E], D4AKK, DL1KO [CB1959][G],

soldier in WW1, businessman (Seeversicherungsvertreter), bio in [CQ-MB 7/1937]

EK4QU, EK4CQ

EK4XP

Gerhard Fröse (- 1975), Hannover Kiel Berlin, DE0154 [E], D4NAQ, D4BLK, D4JQX, D4JQK, D4JQV, DL1GD [E], Gerhard Fröse, Haus Delling, Barsbek b/Schöneberg [CB1951][E][U], elected as WAC member [CQ-MB 8/30]

Adolf Ehni (1911- 1993), near Hannover Braunschweig, DE0702A, D4BBQ [E], D4BCK [E], D4FND [E], DA5IO,

Shack picture, DL1SX [E], [DJ3JD], D4BBQ heard overseas [CQ-MB 10/31, 1/32], WAC diploma 1932 [CQ-MB 2/34] $[\mathrm{C}]$ ??

EK4JO, Albrecht Leyn, D4QE, Hannover [E][V]

Walter Arnold (-1963), Hannover Braunschweig Leimen Heidelberg, DE1377D, D4MFQ, D4FQD, D4MFQ, D4BEK, DA1DP, DL1LL [G], D4QF signals heard overseas [CQ-MB 2/31]

Helmuth Theyson, Hannover, DE0776K, D4NGQ, D4BKK [E], D4JPK, later worked at Paul Tröster Maschinenfabrik?

[G], WAC diploma 1933 [CQ-MB 2/34]

Heinz Schulze (-1966), Erlangen, DE1219, D4BQQ, D4BRK, D4FZF, DA2FF, DL1FE [G][CQ-MB 7/27]

Dipl. Ing. Ernst Franzen, Hannover, Oldenburg, DE0034, D4DJQ, D4BIK, D4JOF 1938 [CQ-MB 7/27][J:3][G][CB],

photo in [CQ-MB 6/30][D]

Walter Ossenkopp (-1963), Celle Hannover, DE0703, DA5HH, DL1VE [E], [G]

Dipl. Ing. Harry Meinel, Hannover, DE0047, DE0349, D4MLQ, D4CEK, D4AQX, D3AQK 1938 [G][V]

near Magdeburg, $[\mathrm{C}]$ ??

[CQ-MB 7/27] ??

$[\mathrm{C}] ?$ ?

near Hannover $[\mathrm{C}]$ ??

Friedrich Kitzinger (-1957), Berlin, Detmold, Karlsruhe München, DE0706, DE1854, D3APF, DA3AP, DL1SI [G]

August Wilhelms, Hannover, DE0772K, EK4QV, D4ORQ, D4CAK, D4LFF?, D3ATK [G][C]

Rudolf Asbach, Hannover, DE0708, EK4PX, EK4CQ, EK4KMC, D4GOQ, D4BAK, D4JLK [G]

Gerhard Specht (-1983), Berlin Hannover Westerland, DE1158K, EK4AB, D4PUQ, D4CFA, D4XDA, D4PSS, D4GFK, DL3LW [G]

Martin Heineberg (1911-1979), Brakel im Nethegau Hannover Buenos Aires, DE0752K [E],[C][TUC with photo],

D4QV [C][G] Emigrated in Sept. 1933 with his brother Dr. Oskar Heineberg to Buenos Aires, Argentina because he was not allowed to finish his medical studies or give concerts with his brother (cello and piano). He worked his entire life at

Moritz (Mauricio) Hochschild (Edelmetalle) in Bueno Aires. Oskar settled in Lima Peru. [Forum Jacob Pins]

EK4QR, August Wilhelms

EK4AN(1), Max Vantler

Adolf Westerfeld, Bad Essen Haltern, DE0804K [C], D4BQW, D4BZK, D4KCK [E],[G]

Hannover [E][CQ-MB 7/27], note in [CQ-MB 7/30][QSLs 2 and 12/1928 E] ??

Heinrich Schrader, Hannover, DE0213 [TUC], [G][TUC] (Sender beschlagnahmt)

Hans Reifenberg (- 1952), Hannover, DE0076 [TUC], D4QY, EK4AJ, EK4QZ, EK4WW, D4QWW, D4PWW?, [F:26],

Author in DASD, 1931][CQ-MB 7/31], emigrated to South Africa, ZS2CM H. Reifenberg [CB1946-1952].

Probably his daughter: Edith Reifenberg (1903-1998): ZS2CM Mrs. E. Reifenberg [CB1954], Mrs. E. Ellgring [CB1958],

DJ0EJ Mrs. Edith Reifenberg [CB1962], joined OV Z22 in 1996 [VFDB, 2000], Interview in [Jürgens, 1997])

Walter Naumann (-1934), Hannover, DE0908K [TUC, C,E], D4QY [E], D4RYQ, D4LNP [G], died in plane crash

[CQ-MB 8/34]

EK4QY, Hans Reifenberg

Georg (Friedrich-Günter [G]) Freiherr von Allizar, Barmen, DE0655, EK4ACJ, 'BABY' in telephony [J:FA9/98],

photo in [CQ-MB 6/30]

Wilhelm Hardt, Köln Klettenberg, DE0086 [G]

Wolfgang Assmann (-1999), Solingen Bad Homburg, DE0913H, D4CCR, D4ICR, D4BKH [E], D4BAL?, DL3DC [G], D4CCR heard overseas [CQ-MB 11/31]

Alois Beuker (-1972), Bocholt, DE2415H, EK4IG, D4VFH, DA4RP, DL3AZ [E], [G] Started in 1924. From 1921-1928

Beuker run a car repair shop and a factory for motorcycle engines and complete motorcycles. Then started a repair shop for radios, which was bombed in 1945. After the war he ran a repair shop for TVs and radios [Bocholt City Infos].

Heinz Evertz, Köln, DE0723I, D4RE, D4DER, D4WER(1), relative of Lothar Rhode, DJ5LR [C][G], D4WER signal 
Received overseas [CQ-MB 2/31, 1/32], author in [CQ-MB 12/30, 3/32, 6/32], Eichwellendienst [CQ-MB 3/30], later produced $\mathrm{x}$-tals at Steeg \& Reuter in Bad Homburg, involved in leading Landesgruppe Rheinland of the DASD, in 1933 VDF members agitated against him [K:103]

EK4RF(1) EK4RE, Heinz Evertz [G]

$\operatorname{EK} 4 \mathrm{RF}(2)$ EK4LF, Richard Fischer

EK4RG Werner Hess, Köln, DE0862I [C], D4EHR [G], D4RHR received WAC [QST 03/1933]

EK4RH [E] EK4BK, Fritz Rinck?, Köln, D4RH, [C][G], qso excerpt [CQ-MB 2/30], Eichwellendienst [CQ-MB 3/30], heard overseas [CQ-MB 11/30], WAC diploma 1931 [CQ-MB 2/34]

EK4RJ Paul Jäger (-1970), Duisburg Berlin-Spandau, DE1339H, DE1639H, D4RJR, D4BHH(1) [E], D4IJH [E], DL7AE [CB1952], DL7PJ [CB1969][G][E]

EK4RK Rudolf Helbig, Köln Bergisch-Gladbach, DE1227 [E], EK4LO, D4NGL?? (hard to read), D4BHI [E], D4IUI, R. Helbig, Berg. Gladbach, Grüner Weg 26 [E][G]

EK4RL

$\operatorname{EK} 4 \mathrm{RM}(1)$

$\operatorname{EK} 4 \mathrm{RM}(2)[\mathrm{E}]$

Eichwellendienst [CQ-MB 3/30] ??

Wilhelm Mummert, Solingen Halensee, DE0976H, D4BMR(1), D4CAH [G]

Dr. Alfred Huppertsberg (-1956/57), Ruhrdistrikt Essen Berlin, DE0288, EK4RP?, EK4HK?, D4CZF(1), D4ACK, D4BMR(2), D4BAH, D4BJF(2) Berlin-Steglitz Brentanostr.47, DL3JG Braunschweig, Retemeyerst. 2 [CB1951] [CQ-MB 7/27][G][C] D4BMR heard overseas [CQ-MB 3/31], author in [CQ-MB 4/31]. He founded (in June 1933) and headed the Verband "Deutsche Funker", a national socialistic amateur radio organisation, unsuccessful in replacing the DASD [K]

EK4RN $\quad$ EK4NK, Bad Kreuznach [C]

EK4RO EK4RR(1), Erich Behrenroth

EK4RP [E] near Köln [C], EK4RM(2)? [G]

$\operatorname{EK} 4 R R(1)$ Erich Behrenroth, Elberufer, DE0781H [TUC], EK4RO, D4KRO, D4CEH [CQ-MB 7/27] [G]

$\operatorname{EK} 4 R R(2)$

EK4RS Hans Ritter (->1995), Wuppertal Barmen Wuppertal-Elberfeld, DE0838H, D4LRR, DL3FP [CB1957], [G][CB]

EK4RU(1) Heinrich Krämer, Köln, DE1254I, D4BSR?, D4BSI, D4ZLC 1938 [G],[CQ-MB 7/27]

$\operatorname{EK} 4 R U(2)$

EK4RU(3)[E]

EK4RU(4)

EK4RU(5)

EK4RV

EK4RW [E]

EK4RX

Karl Mälzer (-1983), Langender Langenberg, DE0476 [C], EK4AF(1), D4MUR, D4PUR, D4BOH, D4IMH, D4STR(1), D4DFC, D4RCC, DL1OE Hauptort. 117, Langender [CB1951][E][G]

Wilhelm Ruloff, Wedel Münster, DE1258I [E], D4RU, D4CBH, D4VFH, Op. at EK4ACK, KR3 [G]

EK4UL(1), Nürnberg [C]

EK4KU(1)

EK4UD

activity reported [CQ-MB 11/30] ??

Ingenieur Otto Wilke (-1963), Barmen, DE0420H [TUC], EK4LZ, D4LZ, D4ACJ, D4BNH, DL6AZ, [G]

Hans Becker (- 1988), Köln-Nippes, Nassmuehle, Kahl (Main), Grosskrotzenburg, DE1236I, EK4FX?, D4FXR [E], D4OEL [E], D4BKI, D4IWI, D4IWR, DL6CV [E], [CB1972-1988][G]

EK4RY

Oskar Becker (1900- 1975), Köln, DE0654, D4LYR, D4JU, D4JUR, D4BAI, D4AII, DL1JU [E], author in

[CQ-MB 3/30], leader of Landesgruppe Rheinland of the DASD, in 1933 VDF members alleged him of former marxistic activities [K:103], policeman (Polizeifunker), NSDAP since 1933, bio in [CQ-MB 7/1937]

EK4RZ [E]

$\operatorname{EK} 4 \mathrm{SA}(1)[\mathrm{E}]$

$\operatorname{EK} 4 \mathrm{SA}(2)[\mathrm{E}]$

EK4SAG EK4GR, Erich Wagner, D4SAG "the wave from the owlmountains" [E] Rheinland, D4RZ [C], heard by PL297, 11/1931 [Krotkofalewski Polski 12/1931] ??

EK4FA, Bernhard Schosinsky (-1985), Hamburg, DE0175, EK4TB, DL1ZU [E], [CQ-MB 7/27][U][HH]

$\operatorname{EK4SAR(1)~[E]~}$

EK4SAR(2)

EK4SAR(3)

EK4SC

EK4SH

EK4SK

EK4GR, Erich Wagner, D4SAG "the wave from the owlmountains" [E]

Julius Kron (-1957), Saarbrücken, later Berlin DE0181, D4AH, D4SAR, D4IAH, D4BFF, D4GEF, [CQ-MB 7/27,
Author in [DASD, 1931], died 1957 in Caracas, Venezuela [J:7][CB1932], WAC diploma 1931 [CQ-MB 2/34]

EK4GR, Erich Wagner

Rudolf Rümer (-1976), Worms Flensburg, DE0284T, DL2ZO [E], [G]

Hermann Franke (-1965), Saarbrücken, DE0441T, TS4SAA, EZ4SAA, D4BCS, D4BTT, D4QDT, DL8AK [G][CB1959]

Fritz (Kurt) Hort (-1929), Friedrichsthal Saargebiet, DE0436T [E][G]

EK4SL [E]

Georg Klos, Saarbrücken Neukirchen, DE0480T, EK4KK, TS4SKL, EZ4SKL, D4BUT(1), D4QCT [G]

[CB1932,1934,1938]

EK4SM(1) [E] Ernst (Bernh.) Dittmar (-1976), Friedrichstal, DE0628T, D4SM [E], TS4SBR, EZ4SBR, D4DMS, EZ4AA, 9S4AD, DL8AD, F4SM [E][G]

EK4SM(2) EK4KA, Dr. Georg Weiss (-1980), DE0327, Kassel, [C][CQ-MB 7/27], EK4WX, DF3FS [G]

$\operatorname{EK} 4 S R(1)$

Alfred Woerner (-1965), Saarbrücken, DE0440T, K4SA, TS4SAX, EZ4SAX, D4BRS, D4BST, D4QET, D4QBT, EZ4Y 9S4AX, DL8AX [G][E]

EK4SR(2) Alfredo della Bona (-1984), DE0453, TS4SAC(1), FS4SAC, EZ4SAC [J:7] Gebrüder Della Bona Vegetables and Fruits Imports, Saarbrücken, founded 1923, Alfredo became CEO in 1930 [CB1932][G][C]

EK4SSA

EK4SW

EK4SY

EK4GB(1), Richard Schönbrunn, Radio-Techniker, Kurzwellengruppe Liegnitz, DE0062 [CQ-MB 7/27]

EK4SZ EK4SZ, Fritz Meyer-Buchhardt

Fritz Meyer-Buchhardt (-1959), Saarbrücken, DE0982T, EK4SY, TS4SAZ [E], EZ4SAZ, D4MYS, D4QFT, 9S4AZ, DL8AZ, Halbergstr. 35, Saarbrücken 3, director of NORAG-Hamburg in 1926 [U][CB1932, 1934, 1951]

EK4TA Adolf Hohenner (-1976), Berlin Lankwitz Flensburg, DE0627F [TUC], EK4AN, D4NNA, D4BMF, D4BNV, DL7AL [CQ-MB 7/27][G] 
EK4TB

EK4TC [E]

EK4TD

EK4TE(1)

EK4TE(2)

EK4TF

EK4TG

EK4TH(1)

EK4TH(2)

EK4TJ

EK4TK

EK4TL [E]

EK4TM

EK4TO

EK4TP

EK4TQ(1) [E]

EK4TQ(2)

EK4TR

EK4TS

EK4TT

EK4TTS

EK4TU

EK4TV(1)

EK4TV(2)

EK4TW

EK4TY [E]

\section{EK4TZ(1)}

EK4TZ(2)

EK4UA(1)

EK4UA(2)

EK4UB

EK4UC

EK4UD

EK4UE

EK4UF(1) [E]

EK4UF(2)
EK4FA, Bernhard Schosinksy (-1985), Hamburg, DE0175, EK4SA, DL1ZU [E], [G]

Dipl. Ing. Werner Manecke (- 1958), Hamburg, DE0012J, EK4OA(1), D4OCT, D4BNN, D4GYF, D4WAO(1), D4CDF, DL1VF $[\mathrm{C}][\mathrm{G}][\mathrm{HH}]$, elected as WAC member [CQ-MB 10/30], D4WAO signal received overseas [CQ-MB 2/312, 1/32] Karl Friedrich Kleefeld (-1974), Kiel Pinneberg Halle Hamburg Berlin Neustadt-Glewe, DE1089V, ex EK4TE, D4ITD, D4BXJ, D4BKJ, D4KNY, D4KNV, D4BIV, D4KNF 1937 [G], in 1935 SA Brigade Marstall, Schwerin. After the war he worked as TV engineer and run a business importing TV sets to Germany [Spiegel 6/7/1950]

Heinrich Retter (- 1956), Hamburg, DE0959J, D4ZET, EK4TV(2), D4QVT, D4BAJ, D4AJJ, D4BJJ, D4RVT, DL3QE $[\mathrm{G}][\mathrm{E}]$

EK4TD, was former call

Lorenz Röhling (-1967), Kiel, DE1622V, D4AFT, D4BDV, D4JDV [E], DA4KR, DL1FM [G]

Hamburg, Shack photo [E] ??

Carlos Retelsdorf (- 1993), Hamburg Coatepec Cuernavaca, DE1644M/U, D4TH, D4CHT [E], X1CZ [E][CQ-MB 7/34], XE1CZ [CB1939], XE1CD [E][CB1993], [G], WAC diploma in 1931. The son of a coffee plantation owner in Coatepec, He operated as "Glenn" during WW2 in Mexico for George Nicolaus, "Max”, and Baron Friedrich Karl von Schlebrügge For operation Bolivar of the German Abwehr, handling coded messages (without knowing the content or being compensated) between Germany and submarines. His license X1CZ was revoked in July 1941 [D]. Another ham involved in this espionage operation was Guido Otto Moebius, XE2CK, XE2IK [CB 1939] whose license was also revoked.

Retelsdorf and Moebius were both in 1942 detained in a prisonor camp in Perote, Veracruz. The german spy operation in Mexico continued using secret messages in letters containing microdots made by Arnold Karl Franz Joachim Ruge.

D4CHT heard overseas [CQ-MB 10/31]

EK4TW, Heinrich Hees [G]

Johannes (John) Gindele (-1986), Hamburg, DE0324 [E][TUC], D4HJT, D4EJT(1), D4BXJ, D4KYJ, D4KYK, DJ2OY $[\mathrm{G}][\mathrm{HH}][\mathrm{CB}]$

Ferdinand Klingenberg, Harburg, DE0753J, D4NKT, D4BJJ, D4KJJ [G][HH]

Hamburg [C][V] ??

Hans A. Münster (- 1985), Leipzig Hamburg Münster, DE0659U, D4LMT, D4BVJ, D4KXU, DJ2FZ [G][HH] [CQ-MB 7/27]??

EK4KMA, Erich Tripmacher, Hamburg?, D4RTA(1), D4CTJ, DL3DQ(1) [HH]

Ernst Suhl (-1983), Holstein Wohnschiff Milwaukee, DE0745, D4TQ, D4LQT [E], D4BBV [E], D4TBV, D4JCV, DL1FQ $[\mathrm{E}],[\mathrm{G}]$

EK4IC(1), Dr. Gerhard Baez

Heinrich Peters (->1994), Hamburg Berlin Apensen, DE0979J, D4ART, EK4CW, D4RVP, D4CGJ, DA6RY, DA6KN, DL3SJ [E], [G][HH]

Erhard Keltsch, Hamburg Braunschweig Berlin, DE0980K/D, D4CWF, D4CNK, D4CND(1) [G]

Arno Brämer, Gumbinnen, DE1422, D4QRE, D4BLA [OP], D4ONE [G?], D4BLA heard by J2QX [QST 6/1934]

[CQ-MB 7/27] ??

Erich Klingenberg, Waren, Mecklenburg, near Berlin Breslau?, DE1121J, DE5308H, D4TU, D4RUT [E],

D4BFJ [TUC], D4IIJ [V], member FWGM [G]

Wilhelm Sattler (- 1990), Harburg, DE0885J [E], D4CFT, EK4FT, EI6FQG, EI2TQ, DF3XX [G]

EK4TE(1), Heinrich Retter, Hamburg [G]

Heinrich Hees, Hamburg Bergedorf, DE0871J, D4CHT(2), EK4TH, D4RWT, D4BCJ, D4KDJ [G]

Wolf Emil Franzok, Berlin Leipzig Lübeck, Haffkrug, Alta Gracia Argentinien, DE1135F, D4LYT, D4GZF, D4FGF, D4CGF, D4CYJ [HH][G][D] worked until 1942 for Telefunken in Rio de Janeiro. In 1943 he started German espionage Activities in Argentina alias "Gustav Utzinger, Luna, Don Antonio, Federico Parker, Juan Stewar(t)" for operation Bolivar of the German Abwehr, led by Johannes Siegfried Becker, "Sargo". With Benno Sobisch, Franzok built the radio networks Red Bolivar and Orga-T. These networks also included Hans Muth, PY1HP [E], a former employee of Siemens-Schuckert in Rio de Janeiro, and Hans Blume, CE4AH [1958, E], who came to Chile in 1927 and was detained in Argentina in 1942. After several arrests in Argentina in January 1944 Franzok (Utzinger) and Becker were forced into hiding. In August 1944, Franzok and most remaining operatives of Bolivar were detained, de facto ending the operation [Hilton, 1999]

[McGaha, 2009]. After his release, Franzok spent the rest of his life in Cordoba, Argentina. Announces broadcasts designed for amateurs [QST 2/37 p.57]

Werner Hensel (-1977), Lübeck, Hamburg, DE0391J, DE0891J [G][HH]

EK4GX, Dr. Erich Wiedemann, Breslau, DE0327, D4AZT [G]

Otto Glaser (-1968), Augsburg Neustadt Haardt, Bad Dürkheim, DE0760T [C], D4RAU, EK4OL, EK4VA, D4BAZ, D4BBT, D4OYT [E], D4CKT, DL9HX [E],[G]

EK4AU, Rudolf Romeike, Berlin, [CQ-MB 7/27][E]

Dipl. Ing. F. W. Behn (-1937), München, DE0470P, D4TMP(1), D4CZF(2), D4ACX. Author in [DASD, 1931]

[CQ-MB 1/32][G]

Anton Plabst (- 1954), Gräfelfing München, DE1277P, D4FCU [E], D4TJP, D4BIP, D4BIB, DL3PO [G][V]

Rudolf Insam (1902-1971), München Stuttgart Lauf, DE0092P/N, EK4RU(5), EK4UL(3), D4IDU [G][DL7AY letter]

He joined 1939 the model train company TRIX [Modelleisenbahn 8/1971][Zschaler, 2000]

Hans Engesser, Schliersee, DE1143P, EK4UF, D4NEU, D4BCP, D4MWP [C][G], 10m activity reported in [CQ-MB 2/31]

Dr. Ferdinand Daser (1907-1996), (Zamboni), München, DE0607P, D4LFU, D4UAW, D4BJP, DL3MCF, started 1927,

[OOTC2641][G][E][CQ-MB 7/27]

EK4UE, Hans Engesser 
EK4UH

EK4UI [E]

EK4UJ [E]

EK4UK

EK4UL(1)[E]

EK4UL(2)

EK4UL(3)

EK4UL(5)

EK4UM(1)

EK4UM(2)

EK4UN(1)

EK4UN(2)

EK4UO [E]

EK4UQ

EK4US

EK4UT

EK4UU(1) [E]

EK4UU(2)

EK4UW

EK4UY

EK4UZ

EK4VA(1)

EK4VA(2)

EK4VB(1)

EK4VB(2) [E]

EK4VD

EK4VE

EK4VF

EK4VG

EK4VJ(1)

EK4VJ(2) [E]

EK4VK(1)

EK4VK(2)

EK4VL

EK4VN

EK4VO

EK4VQ [E]

EK4VR [E]

EK4VS(1) [E]

EK4VS(2)

EK4VS(3) [E]

EK4VT

EK4VU

EK4VW

EK4VX

EK4VY

EK4VZ [E]

EK4WA(1)

EK4WA(2)

EK4WB
Dipl. Ing. Viktor Gramich (-1935), München, DE0115P, EK4UAH, KW3, D4EUH, D4BAP [G], photo in [CQ-MB 5/30] Luwo Schäfer (-1972), München, DE0920, DE0940, EK4UY, EK4XO, EK4OC, D4FOX, Op. D4UAI, DJ1IM [C][V][E] Karl Rittmann, München, DE0759P, EARI, D4JJU [E], [C][E][U], signals heard overseas [CQ-MB 2/31]

Karl Spann, Augsburg, DE0896P [TUC][E], D4PKU(1), D4BHP [G]

Johann Kagermaier, Rosenheim Seebruck Traunstein, EK4RU(3), DE1278P, D4OLU, D4OLO, D2DG [RL-D2 1935], DL3VE, Op at: D4UAM, D4UAW, D4UAZ [G], Nürnberg, EK4RU [CQ-MB 7/27][C] ??

EK4ZA ??

EK4UD, Rudolf Insam

D4UL, $1929[\mathrm{~V}]$ ??

Ludwig Merkl, München, Berlin Charlottenburg, DE0167, D4NMU, D4BSP, D4ZCF 1938 [G]

Herbert Lange, Löbau/Oberlausitz Chemnitz, DE0859M, D4BUM, D4BJM [E], D4LHM,

station photo D4BUM [TUC],[G]

Wilhelm Brandl, Augsburg, DE0897P [TUC], D4DUN, D4BKP, D4DDZ, D4DCZ [G],

near Munich [C]

EK4ZU, Karl Herbig

F. Schwarz, München [C], author "4uo" in [CQ-MB 2/30], WAC diploma 1930 [CQ-MB 2/34]

Dipl. Ing. Ludwig Freiherr von Türkheim-Geisler, DE0002, D4PUQ(2), D4BQU, D4UAJ, D3CRE, EK4UAC [G]

Alexander Schmidt (- 1967), Augsburg Bremen Hanau Kitzingen, DE0768, D4RSU, D4RUS, D4CAT [CB1934][E],

D4BQR, DL1PH, emigrated 1934 to the Philippines, Mobil Oil A. G. Bremen, near München, Bayern [CB1962/65],

new holder in [CB1969],[C][G]

[CQ-MB 7/27] ??

Ing. Johann Steinhauser (- 1957), Oberbayern München Herrsching am Ammersee, EK4UU [E], D4UU [E],

D2DJ [RL-D2 1935], DL1UD [CB1957] (phonie) [CQ-MB 7/27]

EK4HH, Kurt Hartenstein, München, DE0082, D4BUU, see EK4LM [G]

Anton Pfeffer (-1989), Frankfurt, DE0268, D4UAO [G]

EK4UI, Luwo Schäfer [U]

EK4UF, Dr. Ferdinand Daser (Zamboni), München, DE0607 [C][G]

EK4UA(1) [CQ-MB 7/27]

EK4VX, Otto Depser

Heinz Schelbert (-1974), Würzburg Nürnberg, DE0834R, D4KBV, D4BRR, D4ODR, DL1FC [G]

München, [C] ??

Adolf Weigold (1910-1987), Bayreuth Alttrebbin/Oderbruch, DE0836R [TUC], EK4ND, D4NDV, D4BFR, D4NSR,

DL1NR [RL1985], [OOTC 2678][DIG Members][G]

Helmut Kehr (-> 1994), Erlangen, DE1619R, D4NEV, D4BPR [E], D4ACR, DA2AC, DL1EN [E], [G]

Walter Dieminger (1907-2000), München Lindau/Harz, DE0240, EK4UAB, D4UAB, D4UAK, D2DS [RL-D2 1935],

DI2BC, DL6DS [E], Later he was director at the Max Planck Inst. for Aeronomie in Lindau [W], author in

[CQ-MB 10/30, 11/30, 6/31][DASD, 1931]

Fritz Graf jr. (- 1990), Bamberg, DE1495, D4BBV, D4NYR, D4BLR, DL1EJ [G]

Karl Oechsner (-1978), Würzburg Rottendorf, DE0622R, D4RVJ(1), D4OAR, D4BOR, DL1ET [E], [C][G]

near Passau [C] ??

Karl Rückert (-> 1995), Würzberg, DE0618, D4RKV, D4OER, D4BSR, DL1FA, Op. Kv8, EK4UAO [E][G]

Kurt Jäger, Würzburg, DE0636 [TUC], [G]

EK4VK, near Würzburg, Bayern [C][G]

EK4NF, Dr. Ludwig Fauldraht, Darmstadt [G]

bei München, QSL via KY4, [E][CQ-MB 7/27] ??

Hans (Hannes) Bauer (-1987), Nürnberg, DE1147R, EK4VU, D4KAY, D4ARR, D4BAR, D4AUV, D4AQN,

D4UAN, DA2AR, DL1DX, Amateur Radio Shop [G]

Fritz Weingärtner (->1994), Würzburg Marloffstein Berlin Karlsruhe, DE0609F/R, EK4CX, D4BRV(1), D4NRF, D4BER, DA4AW, DL1FI [C][G], D4BRV heard overseas [CQ-MB 7/31], WAC diploma 1933 [CQ-MB 2/34][QSL 1927 E]

Ing. Friedrich Steinlein (Fritz) (-1943), Bayreuth, Nürnberg, DE0413R [E], D4BSV, maybe D4RSV [E], D4BBR [E],

D4NPR(2) [E], [G][E]

EK4EY, Dr. Herbert Schultz (-1984), Rotenburg [G]

near Coburg [E] ??

Fritz Struller (1909-1942 kia), Backnang Ellenbogen Oberkrumbach, DE1159, D4STV, D4BKR [E], D4NXR [G][E]

EK4VQ, Hans (Hannes) Bauer

Würzburg [E], communicated with SP3EM, 11/1931 [Krotkofalewski Polski 12/1931] ??

Otto Depser (->1994), Ansbach Dornberg, DE0984R, D4CXV, EK4VA(2), D4CXZ, D4BJR [E], D4NWR, DL1EB [G]

D4CXZ heard overseas [CQ-MB 5/31, 9/31]

Georg Schuerer, Würzburg, DE0637, D4BYV, D4BHR 1935 [G]

Paul Meisel (-1997), Hof Bayreuth Nürnberg, DE0824R, D4PMV, D4CZV [E], Op. at D4UAX, D4BDR, DA2VZ,

DA4VZ, DL1ES [C][G][V][former calls on DL1ES 1958 QSL, E], station photo of D4CZV [TUC],

Heinrich Schünemann (1909- 1993), DE0815F [C], D4WA, D4EAW, D4BRF [E], D4GOF, DL7CX, Amateur Radio Shop

in West Berlin

EK4AA, Joachim Börner

Rudolf Bassinger, Berlin München, DE1207F, D4FRF 1936 [G][CQ-MB 7/27] 
EK4WD

EK4WE

EK4WF

EK4WG

EK4WH

EK4WI

EK4WJ

EK4WK(1)

EK4WK(2)

EK4WL(1)

EK4WL(2)

EK4WM(1)

EK4WM(2)

EK4WN

EK4WO

EK4WP

EK4WU

\section{EK4WW}

EK4WX(1)

EK4WX(2)

EK4WZ

EK4XA

EK4XB(1)

EK4XB(2) [E]

$\mathrm{EK} 4 \mathrm{XC}$ [E]

EK4XD

EK4XF

EK4XG(1)

EK4XG(2)

EK4XH

EK4XI

EK4XL

EK4XM

EK4XN [E]

EK4XO

EK4XP

EK4XR

EK4XS

EK4XU(1) [E]

EK4XU(2)

EK4XV [E]

EK4XW

EK4XX

EK4XY(1) [E]

EK4XY(2) [E]

EK4XZ

EK4YA

EK4YO

EK4YT [E]

EK4YW
Johannes Rode, Berlin Kiel, DE1157F, D4RDW (twister in [G]?), D4BFV, D4CDF, D4JHV [G]

heard in England [QST 8/1929]

Hans Fechner, Berlin, DE1212F, D4FFW, D4BCF, D4AFF [G]

Helmut Bürkle (->1994), Berlin Ulm, DE1162F, D4BWG, D4GRF, D4BXF, D4CIF, D4BBW, DA7UJ, DL7AQ [G], author [Bürkle, 1950]

Gottfried Kaiser, Berlin, DE1291F, D4MHW [E], D4BNF, D4GLF [E], [G]

EK4CY, Dipl. Ing. Kurt Schlupp, D4GIW

Walter Wechsung (->1994), Berlin Frankfurt, DE1213F/T, D4RJW, D4BWF, D4CWF, D4CWP, DL6VS [E], [G]

Hermann Hopf, Berlin Schmolz, DE1288F [E], D4PKW [E] [G]

Walter Lemm (->1995), Celle, DE2205K, DL1QR [E], [G]

Sebastian Huber (- 1970), Köln Junkersdorf, DE1570I, EK4EKL?, D4BWI, D4MEL, D4QVI, DL1WN [CB1951][G][E]

EK4CC, Dipl. Ing. Erhard Graff

Alfred Noack (-1976), Berlin, DE1294F [TUC], D4LMW, D4BOF, D4ANF, DL7BN [G][CQ-MB 7/27]

München, QSL an K4LD 1926 [TUC] ??

EK4AA, Joachim Börner, D4WN [C]

EK4IB, Fritz Löscher, D4MOW [E]

EK4DZ, Gerhard Thomas, communicated with CV5BJ, 11/1931 [Krotkofalewski Polski 12/1931]

Baurat Hans Klotz (-1980), Berlin, DE1074F [TUC,C], D4AFF 1929 [mentioned on his DE card, TUC], D4ALC,

D4BPA [E], D4BDF [E], D4GDF [E], D4NPA [G]?, [C][E]

[heard Wireless World 12/1925]

EK4QY, Hans Reifenberg [G]

EK4KA, Dr. Georg Weiss (-1980), DE0327, Kassel, EK4SM(2), DF3FS [G][C][CQ-MB 7/27]

EK4CR, Werner Slawyk

Bruno Garnatz (1901-1978), Berlin Marienfelde, DE1263F, D4AZW, D4BGF, D4AQF, DL7BG

[G][DARC District Berlin, 2015]

?, Stuttgart, DE0832 [G] ??

Manfred Bayer, Stuttgart Erfurt, DE0962N, D4BBX, EK4BX, D4BBN, D4LTL, D4LTN [E] Regierungsrat [G]

Heilbronn am Neckar, ex EK4BK [C][CQ-MB 7/27] ??

90KM south of Stuttgart, K4XC, QSL via KY4 [C][CQ-MB 7/27] ??

Alfred Stützel-Sachs (-1966), Aalen, DE0813N [E], D4NDX, D4BQN, D4BAN [G]

Dipl. Ing. Kurt Lederer (- 1990), Stuttgart, DE1504N, DE1594, D4GFX, D4BCN, D4BON, DA1AS after 1945, DL1CW, [F:39], radio repair shop, in 1951 bird-dogging as "KIBITZ 14" in CIA "stay-behind operation KIBITZ", but "had no time"

Shack photo from 1928 [K, page 77] [Schmitt-Enboom \& Stoll, 2015][Lecorte, 2015][E]

Stuttgart, D4XG [E] ??

EK4XY, Martin Gross

Dr. Ing. Ernst Breuning (1910-1942 kia), Stuttgart, DE0458N, D4GHX, D4MFF, D4MFN, D4BMN [G], xtal controlled transmission [CQ-MB 2/30]

Karl-Wilhelm Fischer (-1937), Rottweil, DE0720N, D4RIX, D4BPN, D4MJN [G]

maybe EK4AL(2), see [K, pages 75 and 237][CQ-MB 7/27]

Dipl. Ing. Rudolf Mertz, Stuttgart, DE0027, D4MMX, EK4YAA, D4YAA, D4BAN [G], Author [Mertz, 1927]

EK4BX(1), EK4FN, D4FN, D4XN [C], heard overseas [CQ-MB 11/30, 5/31, 9/31], WAC diploma 1929 [CQ-MB 2/34]

EK4UI, Luwo Schäfer

Kurt von Perglas, Oberhollenhof, Baden Baden, DE0916, EK4PX(3)? Typo in [G], D4XP, D4SPX [G]

likely to be identical with Konrad Kurt Moritz Freiherr Pergler von Perglas (1905-1953) [geni.com]

Erwin Bauer stud. rer. el., Stuttgart Vaihingerstr. 28 Möhringen, DE0456T, D4BGT [E], [CQ-MB 6/27, 7/27],

QSL [E][Harranth, 1996]

Oskar Sauer, Tübingen Stuttgart, DE0040T, D4BSX, D4BIN, D4LZN, DA1ER [G]

Dipl. Ing. Eberhardt Hundt, Stuttgart, DE0273, [C], in 1967 Director of Foreign Technical Affairs,

Daimler-Benz AG, photo in [CQ-MB 5/30]

Peter Windelschmidt, Ulm, DE1128, DE1248N, EK4CZ, D4RUX, D4SUX?, D4YAC [C], D4YZC, D4BGN, D4LXN

[G][CB] D4SUX heard overseas [CQ-MB 3/31], D4YAC heard by FM8CC 1932 [Journal des 8, No 411, 1932, page 6]

Georg Schlegel (- 1965), Vaihingen, DE1150N, D4SVX, D4BLN, D4MEN, DL3AP [G][E]

[CQ-MB 7/27]??

[CQ-MB 7/27] ??

Martin Gross (-1989), Rottweil, Friedrichstr.29, Fabrikant, DE0093, EK4XG, D4LGX, D4BHN, D4LYT, D4OYZ, D4LYN

Standort: Braunschweig, DK9LY, DL6EY [C] Shack [E], (phonie) [CQ-MB 7/27], author in [CQ-MB 2/31, 8/32],

WAC diploma 1930 [CQ-MB 2/34]

Hans Heuwinkel (-1974), Berlin-Spandau, D4XY qsl used 1934! [E], DL7FH [E]

EK4XI [CQ-MB 7/27][G]

[CQ-MB 7/27] ??

Deutscher Funktechnischer Verband, Berlin [QST 12/27, p. 68], heard by W2JD [QST 4/1929 page 84], heard overseas

[CQ-MB 2/31]

?, Stuttgart, received PY1AA on $28 \mathrm{MHz}$ in Oct 1929 - first reception of South America on $10 \mathrm{~m}$ in D

[QST 5/1935, page 11], D4YT heard overseas [CQ-MB 2/31, 5/31][D4YT 1929 QSL to VK3BG, E] ??

[CQ-MB 7/27]?? 
EK4YZ [CQ-MB 7/27]??

EK4ZA [E] Augsburg [C], near Ingolstadt, D4UL, D4ZA [C][E] ??

EK4ZN EK4GJ [G]

EK4ZO Gerhard Bussler (-1972), Danzig Ravensburg Zarneko Eutin, DE0676 [E], EK4ZDG, D4AWY [KFL1944], YM4AA, YM4ZO, DA6ZO, DL1IB [CQ-MB 7/27][G][S]

EK4ZU Karl Herbig (-1979), Nürnberg, DE1112, EK4UN, D4ZU, D4NUZ 1932, D4UAB 1933, D4NQR 1935-39, D4AMV, D4WAU, DA2ZU, DL1EK [CQ-MB 7/27][J:9][V], D4NUZ heard overseas [CQ-MB 2/31]

EK4ZY EK4XY, Martin Gross

EK4ZZ heard overseas [CQ-MB 2/31]?? 


\section{1c) Foreign calls of EK operators and DE holders before 1929, some other unlis stations:}

EAAA

EACM

EAHK

EAJI

EAKL

EAKS

EAKY

EAMM

EAMP

EAPF

EAPP

EAPX

EARA

EARI

ED7MT

EG2IY

EG6CL

EG6FY

EG6PP

EJ7DD [E]

EJ7OO [C]

EJ7WR [C]

EJ7XX [C]

ER5AF

ET1B [C]

ET1E

ET1F

ET2AS

ET2UA [E]

ET2XA [C]

ET2XQ [C]

ET3XY

EWAI

EWBF

EWHI

EWKI

NU2BDQ

SFUP

SMUF

SMZZ
Dr. Josef Fuchs, Wien, DE1004S, UOAA, OE1JF, D4ZWF, [G]

Carl Martin, Wien, DE1083S, UO1CM, OE1CM, [G]

Dr. Otto Kermauner (-1986), Graz Villach, DE1096W, UO5UK, UO6XT, OE6OK, OE6ORK, OE4CW, D4UMW, D4BCW, D4BXW [G]

Fritz Haas (-1981), Wien Beeumont, DE1103S, DE6507, OE1FH, VK6FH [G]

Wilhelm Blaschek (-1964), Klosterneuburg, DE0889W, UOKL, UO3WB, OE3WB, OE3CC, [G]

Franz Schmidt, Wien, DE1012, OE1CS, [G]

Erich Kohout, Wien Zürich DE0698S, UOCX, HB9AT, went to Switzerland in 1934 [G]

Erwin Heitler, Wien DE1079S, OEMM, OE1ER, OE0IO, [G]

EK4MP, Austria

Franz Polacek, Wien, DE1097, DE6468, OE1FP, [G]

Julius Elbert, Graz, DE0452S, [G]

EAHK

Josef Biberauner (-1992), Hausruck, DE1579S, UO5JB, OE5JB, [G]

EK4UJ

Eric Poulsen, Kopenhagen, DE0685, OZ7MT, [G]

A. C. Wilberforce, Köln Bayertal, DE0567, DL2IY, [G]

J. Clarrycoats (-1969), DE0625, G6CL, [G]

F. B. Fereday, London Hone/Sussex, DE0852, [G]

M. W. Pilpel, London, DE0831, [G]

Stjepan (Stefan) Liebermann (1904-1942), Zagreb Croatia Yugoslavia, DE0563, UN7DD, YU7UU [E], He started unlis Operation in 1927 and on a 1928 QSL described himself as unlis, unmarried, a lawyer, and speaking Croatian, French, German and English. By 1930 he had worked over 70 countries and was a member of the WAC club. On a 1933 QSL he spelled his call as: Yugoslavia - 7 - Ultra - Unlis. In 1939 he was the most famous YU station, and listed in [CB1939] as contact for handling QSLs to YU under cover. In 1942 he was killed in the notorious Jasenovac concentration camp (U Logoru) run by the Ustasha government. The militant and fascist revolutionary organization Ustasha ruled an "Independent State of Croatia" in German-occupied Yugoslavia from 1941- 1945, backed by Hitler and Mussolini. They committed on a large scale atrocities and genocide against ethnic serbs and all groups also under prosecution by Nazi-Germany [G][Torbarina][Jasenovac][HSV Database]

??, Zagreb QSL 1927

A. Orekor, Petrovaradin Ecole Radio [E]

Mladen Torbarina, Dubrovnik, [Torbarina][E]

CV5AF

ET1F

EK4CE, Kowno Lithuania [C]

D4TGR, Lithuania

A. Schwichtenberg, Riga Latvia, DE0805, YL2AS 1930 [C],[G]

ET2XA

T. Lapias, Riga Latvia, DE1061, TLA2XA, ET2UA [C], [E][G]

E. Lindans, Latvia, KC2U [C] 1927, TLA2XQ, [E]

Eugen Tumma (1904-1942), Paernu (Pernau) Estonia, DE0453, TE-XX [C],[G], died after deportation to Russia [ES3GZ] EK4AW, Hungary

EK4AW, Hungary

Kurt Nekolny, Budapest, DE0423A, [G]

Istvan Kemeny, Budapest, DE0736, EWH2, [G]

EK4CJ

Gerhard Senkel, Stolp Langenballig, DE1753B, DL1GS, [G]

Robin Hult, Vadstena, DE0157, [G]

G. Fant, Norwiken, DE0158, [G] 
(2) 1930-1932:

2a) Official "lis" calls - some of them remained in use after 3/1933:

D4AAG

D4AAK

D4AAL

D4AAO

D4AAP

D4AAR

D4AAV

D4ABA

D4ABF

D4ABG

D4ABI

D4ABK

D4ABN

D4ABQ

D4ABP

D4ABR

D4ABV

D4ABW

D4ABX

D4ACA

D4ACI

D4ACJ

D4ACX

D4ADB

D4ADC

D4ADE

D4ADF

D4ADH [E]

D4ADI

D4ADK

D4ADL

D4ADQ

D4ADU

D4ADZ

D4AEF

D4AEL

D4AEN

D4AEO

D4AEQ

D4AER

D4AET

D4AEU

D4AEY

D4AEZ

D4AFA

D4AFC

D4AFD communicated with CV5BJ, 11/1931 [Krotkofalewski Polski 12/1931] ??

EK4AAK(2), Kurt Müller, Liebschwitz/Elster [CB1932]

EK4AAL(2), Ernst Busse, Jena [CB1932]

EK4AAO(2), Max Gerstener, Reichenbach [CB1932]

EK4AAP(2), Kurt Hartenstein, Plauen [CB1932], signals heard overseas [CQ-MB 2/31, 3/31]

EK4AAR(2), Christoph Schmelzer, Lichtentanne/Sachsen[CB1932], Eichwellendienst [CQ-MB 3/32], author in

[CQ-MB 10/31, 10/32], signals heard overseas [CQ-MB 10/30, 3/31], WAC diploma 1929 [CQ-MB 2/34]

EK4AAV, Polytechnikum Friedberg/Hessen [CB1932]

EK4ABA, Radioclub Delmenhorst [CB1932], signals heard overseas [CQ-MB 3/31]

EK4ABF(2), W. Doehring, Karlsruhe [CB1932], signals heard overseas [CQ-MB 2/31]

EK4ABG, Otto Anton Klotz, signal heard overseas [CQ-MB 10/30, 2/31], elected into WAC [CQ-MB 2/31],

station description [CQ-MB 7/30,3/31], WAC diploma 1930 [CQ-MB 2/34]

$\operatorname{EK} 4 \operatorname{ABI}(2)$, Max Drechsler, Halle/Saale [CB1932]

EK4ABK(2), Dr. Heinrich, Wismar [CB1932], xtal controlled transmission [CQ-MB 2/30]

EK4ABN, signals heard overseas [CQ-MB 2/31], WAC diploma 1930 [CQ-MB 2/34]

EK4ABQ, Gustav Bock, Frankfurt/a. M.-Süd, EK4KB, [E], D4BFT 3/1933 [V][CQ-MB 2/32]

EK4ABP(2), J. Stefanski, Frankfurt/Main [CB1932][E], communicated with SP3OL [Krotkofalewski Polski 12/1931]

EK4ABR, F. Boedigheimer, Frankfurt/Main[CB1932], author in [CQ-MB 7/30, 11/30, 9/31], photo in [CQ-MB 6/30], communicated with SP1AT, 11/1931 [Krotkofalewski Polski 12/1931], crystal controlled [QST 6/29 p.67][E]

EK4ABV, Verein der Funkfreunde Schlesiens [CB1932], 80m activity reported [CQ-MB 8/30],

mentioned in [CQ-MB 10/32], communicated with SP1AH, 11/1931 [Krotkofalewski Polski 12/1931]

EK4ABW, Technische Hochschule Hannover [CB1932]

EK4ABX(2), W. Oehlerking, Hannover [CB1932], heard overseas [CQ-MB 11/31], communicated with SP3OL

[Krotkofalewski Polski 12/1931]

EK4ACA, Verein Ostdeutscher Funkfreunde, Königsberg [CB1932], communicated with SP3OL

[Krotkofalewski Polski 12/1931]

EK4ACI(2), Dr. Schmitz, Muehlheim [CB1932]

EK4ACJ(2), V. Allizar, Barmen [CB1932], heard overseas [CQ-MB 11/31]

EK4ACX, Technische Hochschule Berlin Charlottenburg [CB1932], D2BR-D2BT [RL-D2 1935],

station description [CQ-MB 3/32]

EK4ADB(2), Funktechnischer Verein Gruppe Siemens, Berlin Siemensstadt [CB1932], station description in

[CQ-MB 12/32] by H. Lamprecht, DE1208, EK4AC(2), heard overseas [CQ-MB 10/31]

EK4ADC(2), D4BAF, Laboratory of the FTV and DASD, Berlin [CB1932], WAC diploma 1933 [CQ-MB 2/34],

communicated with SP1AT, 11/1931 [Krotkofalewski Polski 12/1931]

EK4ADE(2), Dr. Ing. Friedrich Trautwein, Berlin Zehlendorf [CB1932]

EK4ADF(2), Wolfgang Rach, Berlin Friedenau [CB1932], elected into WAC [CQ-MB 2/31], heard overseas

[CQ-MB 3/31], WAC diploma 1930 [CQ-MB 2/34]

EK4ADH(2), Funktechnischer Verein, Ortsgruppe Lorenz, Berlin Tempelhof [CB1932], new transmitters [CQ-MB 3/31]

EK4ADI, Kurzwellengruppe Berlin Schoeneberg [CB1932]

EK4ADK ?? [CB1932]

signals heard overseas [CQ-MB 7/31] ??

EK4ADQ, Deutsche Versuchsanstalt f. Luftfahrt Berlin Adlershof [CB1932], heard overseas [CQ-MB 8/31]

EK4ADU(2), Friedrich Dahlke, Liegnitz[CB1932]

EK4ADZ, Kurzwellengruppe Magdeburg [CB1932], heard by PL198, 11/1931 [Krotkofalewski Polski 12/1931]

Rheinische Ingenieursschule, Mannheim, D2CW [RL-D2 1935], [CQ-MB 12/32] Ops.: Rudolf Mirche, Mannheim,

DE1262 [TUC], EK4OD, D4BFO [E]; Erich Walter, DE1056, D4BHO [E]; Franz Koch, Mannheim, DE1053, D4BLO [E],

Franz Ströck, Mannheim, DE1488 [TUC], EK4OG

EK4AEL(2), Flugfunkschule Staaken [CB1932]

EK4AEN(2), Ernst Ramke, Hamburg [CB1932]

EK4AEO, Hamburger Radioclub [CB1932], station description in [CQ-MB 9/31][E]

EK4BR, EK4AEQ(2), Hans Krautzig, Kottbus [CB1932], elected to WAC [CQ-MB 6/30],

uses room in prison [CQ-MB 12/30], WAC diploma 1930 [CQ-MB 2/34]

EK4AER(2), Allg. Transportanlagen A. G., Leipzig [CQ-MB 10/32]

EK4AET, Technikum Mittweida, Op Hans Teuchert (-1963), DE1702, later Dozent an der Fachhochschule für

Elektrotechnik "Fritz Selbmann" in Mittweida, author [Teuchert, 1953] [CQ-MB 10/32]

EK4AEU(2), Karl Winkler Radiolaboratorium, Leipzig [CB1932], D3BVU [CB1938,1939][C]

EK4AEY, Funkverein Braunschweig [CB1932][E]

EK4AEZ, Flugschule Staaken, signal received overseas [CQ-MB 10/30, 2/31, 3/31, 1/32], elected into WAC [E] [CQ-MB 2/31]

EK4CL, EK4AFA(2), Dr. Curt Lamm, Berlin [CB1932]

EK4AFC, Ernst Ramke, Altona [CB1932]

EK4AFD, Telefunken, Berlin [CB1932] 
D4AFE

D4AFF [E]

D4AFG

D4AFH(1)

D4AFH(2)

D4AFI

D4AFJ [E]

D4AFK

D4AFL

D4AFM

D4AFR

D4AGE

D4AGN

D4DBA

D4DBD

D4DBF

D4DBQ

D4DBS

D4DBV

D4DBW

D4DCF

D4DDC

D4DDF,

D4DEA

D4DFB

D4DFK,

D4DFZ

D4DKA

D4DKB

D4DKC

D4DKE

D4DKF(1)

D4DKF(2)

D4DKP

D4DOA

D4DOB

D4DOC(1)

D4DRT

D4KAD

D4KBL

D4KBU

D4KGL

D4KHF

D4KKA

D4KKO

D4KMA

D4KMC

D4KQB

D4KQC

D4KQD

D4KQE

D4KQF [F]

D4KQG(1)

D4KQG(2)

D4KUA

D4KUB

D4KUE

D4KZA
EK4AFE, Dr. P. Lertes, Frankfurt a. Main [CB1932]

Prof. Dr. Heinrich Wigge (1889-1954 [1959?]), Köthen Mölln, DE0603J [G], Trustee at EK4AFF, Professor of Physics and High-Frequency Technology [Schwarz, 2015]. Organized in 1930 with Rolf Wiegand the $5^{\text {th }}$ DASD meeting in Halle/Saale. Author: Die Neuere Entwicklung der Funkentelegraphie ein Siegeszug der Vakuumröhre Verlag der Ingenieur-Zeitung 1921, Rundfunk-Technisches Handbuch, De Gruyter.

EK4AFG, Aeronautisches Observatorium, Lindenberg Kr. Beeskow [CB1932]

EK4AFH, Ingenieursschule Altenburg Thueringen [CB1932]

Ingenieursschule Weimar, D2CP [RL-D2 1935], [CQ-MB 10/32]

EK4AFI, Deutsche Seewarte, Hamburg [CB1932], communicated with CV5BJ, 11/1931 [Krotkofalewski Polski 12/1931]

EK4AFJ, Höhere Maschinenbauschule Neustadt-Glewe [CB1932], Op: Karl Kleefeld EK4TD,

signals heard overseas [CQ-MB 3/31]

EK4AFK(2), Telefunken, Berlin [CB1932]

EK4AFL, Askania Werke C., Bamberg Berlin [CB1932]

EK4AFM, H. Brinkmann [CB1932], signals heard overseas [CQ-MB 7/31]

EK4AFR(2), Telefunken, Berlin [CB1932]

EK4AGE(2), Dr. Trautwein, Hochschule f. Musik, Berlin Charlottenburg [CB1932]

EK4AGN, EK4BY, Berlin, 1929 [V]

EK4DBA, Hugo Fagien (-1953), Königsberg [C]

EK4DBD, EK4DB, Fritz Schnepel, Königsberg, Pr., D4DBD [C][CB1932], signals heard overseas

[CQ-MB 10/30, 3/31][E]

Volker Magdalinski, Frankfurt/Oder, DE1903C, D4KQF [G]

communicated with SP3OL [Krotkofalewski Polski 12/1931] ??

EK4DBS, Gustav Dybus, Königsberg Hannover (Heer), DE0674B, KW5, K4DOS [G]

communicated with SP3OL [Krotkofalewski Polski 12/1931] ??

EK4DBW, EK4ES, Hans Wottrich

EK4DCF, Fritz Weber, Reichswehr, Berlin, DE1861F, D4DCF 1932, D4DFS [J:8][G]

EK4DDC, EK4BY, EK4KZA, Josef Brey, Heer [F]

EK4DDF, G. Ewert, Heer [F]

EK4DEA, Heidenfelder, Stuttgart Bad Cannstatt, DE0011, D4DEA, Mechaniker-Meister [G]

D4BZB [E]

EK4DFK, Oscar Gruber, Münster, Stuttgart Cannstatt, Potsdam, DE0678, EK4LG, D4LGL, D4BQH, D4RRH [G]

EK4DFZ, A. Neunert, Berlin, DE1742F, Heer [F][G]

EK4DKA, Heidenfelder, Stuttgart, DE0011N, Mechaniker Meister, Heer [G][C][F][CQ-MB 7/27],

author in [CQ-MB 11/30]

EK4DKB, Nachrichtensoldaten der Reichswehr, Kassel, D4DKB, Frhr. von Lersner,

verantw. von Uckermann, Kassel, DE1226J [G] [E]

Willi Brüssow, Frankfurt/Oder, DE1088C, D4BFC [E], [G]

EK4DEA

EK4DKF(1), Hans Schilling, Gruben?, Stuttgart [C]

EK4DKF, EK4DFK, Oscar Gruber, ? Heer [F], wkd ZL1FW [QST 6/29 p.67]

Karl Dirnagl, München, DE1906P [G]

EK4DOA, Fritz Scheuering, München, DE1532P [G][CB1932][1929 QSL Op.: E Müller??; E]

EK4DOB, EK4KUA, Hans Link [V], communicated with SP3OL [Krotkofalewski Polski 12/1931][E]

EK4DOC, Adam Schönberger, München, DE2081P, Heer [F][G]

EK4DRT, Ph. Eckhardt, Heer [F]

EK4KAD

EK4KBL, EK4KBU, W. Kilian, Kassel [C][CQ-MB 7/27]

EK4KBU, W. Kilian, Niederzweren b. Kassel, EK4KBL, Heer [F][CB1932]

EK4KGL [CQ-MB 7/27] ??

EK4KHF [CQ-MB 7/27] ??

EK4KKA, EK4BY [G]

Fritz Endress, Kassel Giebelstadt Hannover, DE2436K [G]

EK4KMA, Erich Tripmacher, Hannover, Heer, EK4TP, D4RTA(1), D4CMJ 1928 [V], DL3DQ(1), [F][CB1932]

EK4KMC, EK4QU, Rudolf Asbach, Heer [F]

EK4KQB, Paul Reschke, Osterode Lager Lechfeld, DE1737A, Kavallerie [F][G][V]

EK4KQC, E. Sakschewski, Kavallerie [F]

EK4KQD, G. Ewert (Reiter?), Potsdam, [as EK4KOD in C], Kavallerie [F]

EK4KQE, Ltn. Schrottler, Kavallerie [F][CB1932]

EK4KQF, Hans Schleifenbaum, Kavallerie [F], DEM1721, D4CBC, D4CAM [E], D4CAL(1) [E], D4CAR, DL1YA [G]

EK4KQG, M. Jakob, Kavallerie [F]

D4DBF $[\mathrm{G}]$

EK4KUA, Hans Link, Bamberg Cannstatt, DE1163R, D4ULI [E][V], Kavallerie, EK4DOB, EK4KUR? [F][G][CB1932]

EK4KUB, Heinrich Wurz, Bamberg Langensalza, DE1167R, Kavallerie [F][G][E]

EK4KUE, Otto Rabethge (-1976), Parchim Stettin Kiel, DE1785V/B, D4HUB, D4CLJ, D4CGT, DL1GN Kavallerie [F][G]

EK4KZA, EK4BY, EK4DDC, Josef Brey, Heer [F][CB1932] 
D4LAA

D4LAB

D4LAC

D4LAD

D4LAJ

D4LAL

D4LAX

D4LLD

D4LSG

D4MFM [E]

D4SAR

D4UAA

D4UAB(1)

$\mathrm{D} 4 \mathrm{UAB}(2)$

D4UAC

D4UAD

D4UAG

D4UAH

D4UAI

D4UAJ

D4UAK(1)

D4UAK(2)

D4UAL

D4UAM

D4UAN

D4UAO

D4UAP

D4UAQ

D4UAR

D4UAS

D4UAU

D4UAV

D4UAW

D4UAX

D4UAZ

D4XAA

D4XAB

D4XAC

D4XAD

D4XAE

D4XAF

D4XAG

D4YAA

D4YAB

D4YAC

D4YAD

D4YAE
EK4LAA, Rolf Ehrhardt (-1955), DE1715A, D4CDA, Marine, DJ1HT [CB1953-1955][F][G]

EK4LAB, Bodo Kiesel, Stralsund Hannover Kiel Warnemünde, DE1728V, D4HIF, D4BRJ(2)?, D4BRV(2) [E], Marine $[\mathrm{F}][\mathrm{G}]$

EK4LAC, Werner Arnold (->1995), Pillau Kiel, DE1776A, Sondergruppe M, D4CFA, DL1OB, Marine [F][G]

EK4LAD, Josef Stratenschulte, DE1762A, Sondergruppe M, D4CHA, Marine [F][G]

EK4LAJ, Hugo Wördemann (1915-1999), Cuxhaven Temecula, DE1857J, D4BSS, D4BZJ [E], D3LAM, WB6CJG, K6TE

$[\mathrm{G}]$

communicated with SP1AT, 11/1931 [Krotkofalewski Polski 12/1931] ??

EK4LAX [CQ-MB 6/27, 7/27] ??

EK4LLD [CQ-MB 7/27]??

signals heard overseas [CQ-MB 6/31] ?? EK4GS??

EK4MF [CB1932]

EK4SAR, Julius Kron [CB1932]

EK4UAA, Benediktinerstift St Stephan, Augsburg, KV9 [M 1927][CB1932]

EK4UAB(2), Walter Dieminger, Amberg, DE0240 [CB1932], heard overseas [CQ-MB 5/31]

Karl Herbig, Nürnberg [CQ-MB 10/32]

EK4UAC, Dr. Ing. Otto Papp, Burghausen, Mückenberg, DE0001 [TUC], DE1824P, KW1, D4UAC [E], D4QCP, D4BFP, D4QZU, D4QZP [CQ6/1937], D3CRT Gesellschaft für Missionsfunk, Niederndobrach near Kulm, D2BD [RL-D2 1935], Op: Robert Wunder, [CQ-MB 7/27]

EK4UAD, Dr. Dickmann, a. o. Prof. a. D. Technischen Hochschule München, Gräfelfing, KV1 [M 1927]

EK4UAG(2), Dr. Ing. Hans C. Deckel, Solln, DE1568V/P, D4REZ, D2DH [RL-D2 1935][E], D3BMP [E], He enters the

large milling machine company in Munich founded by his father, the Friedrich Deckel AG in 1928. The company starts to build camera shutters (Synchro COMPUR), he held many patents in this area. Becomes director for shutter and machine Production in 1934, in 1939 CEO and co-owner with his brother Dipl. Ing Fritz Deckel. After the war, gives up amateur radio and keeps low key, but see [Spiegel 51/1956].

EK4UAH, Viktor Gramich, cand. Ing., Versuchsstation Murnau, KW3 [M 1927] Op: EK4UH, Dipl. Ing. Viktor Gramich [C][CQ-MB 7/27][CB1932], 10m activity reported in [CQ-MB 2/31]

EK4UAI, Süddeutscher Radioklub, München, KW6 [M 1927][CB1932]

EK4UAJ, EK4UQ, Dipl. Ing. Ludwig Freiherr von Türkheim-Geisler, München, KW7, D2DE [RL-D2 1935], [M 1927][CB1932],

heard overseas [CQ-MB 5/31]

EK4UAK, Oberleutnant Fritz Behringer, Gudrunstr. 9, München, DE0340 [C][M 1927][V][CB1932]

EK4UAB(2), Walter Dieminger, Amberg, DE0240 [CQ-MB 10/32]

EK4UAL, Anton Freiherr von Massenbach (1899-), München [M 1927][CB1932]

EK4UAM, Süddeutsche Telefonapparate \& Drahtwerke A.-G., Nürnberg, KV7 [E][M 1927]

EK4UAN(2), EK4VQ, Hans Bauer, "Funker Schupo", DL1DX Fürth [C][V][CB1932], 1st 1931 DX qso in 1931

[CQ-MB 4/31], heard overseas [CQ-MB 5/31], communicated with SP3OL [Krotkofalewski Polski 12/1931]

EK4UAO(2), Erziehungsanstalt Ettal, Pater Emanuel Haiss, Kloster Ettal, DE1342P, D4IOI, DL2PE; 2nd OP: Heinrich Haffmann, München Ettal Canada, DE1334P, D4EZU, D4BTP [G][V][CB1932], station description [CQ-MB 4/32], WAC diploma 1932 [CQ-MB 2/34], heard by PL198, 11/1931 [Krotkofalewski Polski 12/1931]

EK4UAP, Städt. Handelsschule Augsburg [M 1927]

EK4UAQ, Überlandwerke Niederbayern, Landshut [M 1927]

EK4UAR, Höhere Technische Staatslehranstalt, Nürnberg, K4UAR [E][M 1927]

EK4UAS [CQ-MB 7/27] ??

heard by PL198, 11/1931 [Krotkofalewski Polski 12/1931] ??

heard by PL198, 11/1931 [Krotkofalewski Polski 12/1931] ??

EK4UAW, EK4UF, F. M. Daser [CB1932], other op: EK4UL(3) [CB1932]

EK4UAX ??, Op: EK4VZ

EK4UAZ, EK4UL(3), Johann Kagermeier (Tuntenhausen), D2DG [RL-D2 1935], [CB1932][CQ-MB 10/32]

EK4XAA, Elektrotechn. Institut der Technischen Hochschule Stuttgart, KY1 [M 1927]

EK4XAB, Physikalisches Institut der Technischen Hochschule Stuttgart, KZ3 [M 1927]

EK4XAC, Zeppelinwerft Flughafenfunkstelle Friedrichshafen (Bodensee) [CB1932]

EK4XAD, Radiokosmos der Franck'schen Verlagsbuchhandlung Stuttgart, KZ0, D2DX [RL-D2 1935], [M 1927] [CQ-MB 7/27]

EK4XAE, Radiowerk A. Laukart, Trossingen, KY9 [J:1924 Versuchsender][M 1927]

EK4XAF, Telegraphen-Werkstätten der O.P.D. Stuttgart [M 1927]

EK4XAG, Dornier Metallbau, Friedrichshafen (Bodensee) [CB1932]

EK4YAA(2), EK4XM, Dipl. Ing. Rudolf Mertz [CB1932]

EK4YAB(2), Fritz Sabrowsky, Stuttgart, DE0101N [CB1932], signals heard overseas [CQ-MB 7/31]

EK4YAC, Funkverein Ulm a. Donau im OFV [M 1927] Op: EK4XU(2), P. Windelschmidt, Ulm a. Donau, DE1128, Bleicherwalkstr. 11 [C], station description in [CQ-MB 7/31], communicated with SP1AT, 11/1931

[Krotkofalewski Polski 12/1931]

EK4YAD, Funkverein Göppingen im OFV, KY7 [M 1927][CQ-MB 7/27]

EK4YAE, Funkverein Rottenburg im OFV, KY8 [M 1927] Dipl. Ing. Rudolf Horkheimer (1894-1982), K4YAE, CP6XF, DK5GA, (phonie) [CQ-MB 7/27] 





D4BOG [E] Victor Menzlewski (-> 1994), Reichenbach Wermelskirchen Frankenstein, DE1299G, 9X4MV, DJ2AZ [G][E][CB1959], heard by PL428, 11/1931 [Krotkofalewski Polski 12/1931], worked by W4ABR [QST 5/1932]

D4BOI

D4BOP

D4BOV

D4BPA

D4BPV

D4BQG

D4BQL

D4BQQ

Willi Schwenke (-1937), Düsseldorf Lohausen, DE1719H, D4BWH(2), D4YNH [G]

heard by PL423 [Krotkofalewski Polski 6/1933] ??

Karl Wrackmeyer, Flensburg, DE1909V, D4BPV [G]

EK4WU

D4BOV

EK4GO

$\operatorname{EK} 4 \mathrm{IM}(1)$

EK4QI

EK4UQ

D4BQW

D4BRB

D4BRH

D4BRL

D4BRN

D4BRP

EK4QW(1), heard by PL428, 11/1931 [Krotkofalewski Polski 12/1931]

EK4BR

W. Hochheimer, Recklinghausen, DE0788 [G], heard by BRS427 [QST 5/1933] and by DE1595 [QSL to D4DLD, TUC] P. Heise, Wiesensee/Thueringen DE1790L [G]

Ferdinand Rüb, Stuttgart, DE0818N, 1932

Ludwig Luther (- 1975), München Marktoberdorf, DE1826P, D4SNP, DL1XS, LGN IP Bayern-Süd, then excluded from DASD, [G]

D4BRS

D4BRV(1)

D4BSG

D4BSO

D4BSR

EK4SR(1)

EK4VR

Käthe Jacobs, Breslau Baden Baden, DE1282G, started 1931 [F:81][G]

EK4HH $(1,3)$

EK4RS

D4LAJ

EK4VS(1)

EK4XS

EK4AT

D4MPR [E]

EK4GU

Heinrich Haffmann, München Ettal Canada, DE1334P, D4EZU, Op. at D4UAO [G]

Arnold Wahl (- 1993), Mainz Ascheberg, DE1754T, D4PMK, DL1HZ [G]

EK4BU(1), communicated with CV5BJ, 11/1931 [Krotkofalewski Polski 12/1931]

D4UWG

D4BUC(1)

$\operatorname{D} 4 \mathrm{BUC}(2)$

D4BUG

D4BUH

D4BUM

D4BUT(1)

D4BUT(2)

D4BUU

D4BVC

D4BVG

D4BVL

D4BVT

D4BWC

D4BWG

Karl Krüger (- 1980), Samen Spremberg Schwarzheide, DE1677C, DE2123, D4RVC, D4RVG [CB1938-39], D4RVJ(2), DM2AEB [CB1954], [G][His 1962 QSL, E]

Fritz Brand (->1995) (also Hans Specht?), Brieg Rodenfeld/Weser Bonn, DE1665G, DL3VX [E], [G],

DL3VXC Elsa Brand [CB1973]

D4ZAR

EK4UM(2), station photo [TUC]

EK4SK

D4NCR

$\mathrm{EK} 4 \mathrm{UU}(2)$

EK4BI(1)

Jonny Rehder, Köln/Riehl, DE1818H/I, D4JZI [G]

Friedrich Externbrink, Halle/Saale, DE1595L [TUC], D4MEH, D4TFL, D4TFU[CB1938], [G]

Leo Altmann, Ludwigshafen Friedheim Gräfelfing, DE1830T [G]

Max Miekley (- 1958), Burg Fehmarn Eberswalde, DE1335A, D4NXB, D4BWV DL3ON [CB1957/58], [G]

EK4WG

Ewald Fischer (->1995), Düsseldorf Braunschweig, DE1574H/D, D4MPL, D4OHL, D3GOH, DL1TZ [G]

D4BWH(1)

D4BWH(3)

D4BWI

D4BWL

D4BOI

EK4WL(1)

Karl Jacobs (-1979), Wuppertal München, DE1541H, DA4DB, DL1OG, ZS6BKJ, [G][E]

D4BWR

D4BWV

D4BXI

D4BXG

D4BXR

D4BXT

D4BXU

D4BYH

D4BYV

D4BZB

D4BZC

D4BZG

Joseph Wilhelm Schüll (-1957), DE1834R [E], D4SWR, DL1FD [G]

D4BWC

D4KZR

EK4GX

Hans Schäfer (-1984), Halle/Saale Erlangen, DE1836R, D4WPP, D4WPR, D4WPU, DL1FB [G]

Friedrich Zasel, Bad Kreuznach, DE1585T, DE2441, D4KZN, D3CJT [G]

EK4MK

Hans Landmesser (-1986), Essen, DE1821H, D3GNH, DJ1IL [E], [G]

EK4VY, Georg Schuerer, Nürnberg, D4BHR [E]

Werner Guhrke, Stettin Koeslin, DE1223B, XD4XDB [E], D4BAB, D4BFB, D4DFB [E], D4TNB [G]

Wilhelm Krüger, Brandenburg, DE1798C, D3GVC [G]

Ernst Gopon, Breslau Bremen Friedrichshof, DE1349G [TUC, E], D4KZG, D4WHG [G] 
Dr. Hansjürgen Raporte (- 1957), Düsseldorf Eichwalde Molkau bei Leipzig, DE1841H, D4VEH [KFL1944], DM2ANM [CB1955-57], [G]

D4CAC Kurt Mehlhorn, Witz/Brandenburg, DE1799C, D3CPC [G]

D4CAL(1)

D4CAM

Karl Eckel (- 1975), Wanne/Eickel Wuppertal, DE1919H, DL1PC [G]

D4CAR

D4CAT

D4CBA [E]

$\mathrm{D} 4 \mathrm{CBH}$

D4CBJ

Bertold Präg, Dresden, DE1596M, D4BCM [G]

D4CBM

D4CBW

$\mathrm{D} 4 \mathrm{CCH}$

D4CCJ

D4CCK

D4CCR

D4CCW

D4CDD

$\mathrm{D} 4 \mathrm{CDH}$

D4CDM

D4CDO

D4CEL

D4CET

D4CFE

D4CFH

D4CFT

D4CGH

D4CGM

Wolfram Greiff, Krefeld, DE1526I, D4CLR, D4HEC(2) [G]

Richard Utikal (-1978), Koblenz Berlin, DE1573T, D4ZUN, D4BAQ, D4CBT, D4OUT(1) 1936, DL7EK [G]

EK4BL(2) [V]

EK4RU(2)

EK4FK

M. Gross, Stuttgart, DE0390N, elected as WAC member [CQ-MB 8/30]

1930, near Berlin [V] ??

Willi Schott, Essen, DE1822H, D4UXH [G]

Robert Grabow (- 1993), Neustrelitz Partz/Wahn Oldenburg, DE1768C, DE1786?, DL1XM [G]

EK4KC

EK4RC

Georg Schmidt, Berlin (- 1955), DE1560F, D4CSF, D4HHF [E], DL7CS [E], [G]

heard overseas [CQ-MB 10/31] ??

D4RUH

Dipl. Ing. Hans Kaiser (- 1980), Berlin Freiberg, DE1748M, D4WSM, D4RYF, D4RYM, DM2ACN [CB1959],

DM2ACO [CB1962-1978],[G]

Erich Linsin (->1995), Mannheim Ladenburg Konstanz Frankenthal Singen Waldshut, DE1829C [E], D4TAO, D4TAT,

DA9KK, DK9KK in 1946, DL6GB [E], on the air since 1929 [G]

EK4LF

EK4NG

Maximilian Bleß, Berlin, DE1562F, D4CVF [E], D4HKF [G]

Paul Wichelhaus (-1978), Venlo Kaldenkirchen, DE1840H, DA4ZB, PA0RU, PA0PR [G]

Lutz Felmer (-1946), Münster Mainz, DE1808H/T, D4RGH [G]

D4ZAR

EK4GN

D4CGT(1)

D4CHT [E]

$\mathrm{D} 4 \mathrm{CIC}(1)$

$\mathrm{D} 4 \mathrm{CIC}(2)$

D4CJM

D4CKK

D4CLR

D4CLT

D4CMA

D4CMC

D4CMU

D4CNW

Dr. E. Ganser, Frankfurt/Main, DE1888T [G]

EK4TH(1), heard by PL428, 11/1931 [Krotkofalewski Polski 12/1931][E]

Georg Schaardt (- 1993), Hamburg, DE1741J, DL9NW [G]

DL7CT

communicated with CV5BJ, 11/1931 [Krotkofalewski Polski 12/1931], heard by PL423 [Krotkofalewski Polski 6/1933] ??

Friedrich Beil (- 1984), Wilhelmshaven, DE1860K, D4FSK, DL1WH [G]

D4CAR

Werner Schulze (-1980), Kassel Hermansrode, DE2190T, DA3PU, DL1AP [G]

D4PSQ

$\operatorname{EK} 4 \mathrm{CM}(1)$

Adolf Clorius, Chemnitz Neustrelitz Timmendorferstrand Rechlin, DE2096J/U, D3CMJ [CB1938] [G]

D4COF [E]

D4CSG

Heinrich Krüger, Berlin Marienfelde, DE1289F [G]

EK4AM [E]

Josef Buhl (-1937), Glatz (Oberschlesien), DE1433G [E], D4BFG 1934, D4HRG, [G]

communicated with CV5BJ, 11/1931 [Krotkofalewski Polski 12/1931], qsl from ON4GQ 1930 [E]

D4CSI

D4CSL

D4CTJ

D4CUF

D4CUL

D4CVC

D4CVT [E]

D4CWA

D4CXJ

D4CXV

D4CZF

D4CZU

D4CZV [E]

D4DAF

D4DAN

D4DDM

D4DEN

D4DER

D4DFR

D4DGN near Berlin, QSL from SP3OL 1931 [E] ??

communicated with SP1AH, 11/1931 [Krotkofalewski Polski 12/1931], worked SP1EB [Krotkofalewski Polski 6/1933] ??

EK4TP

EK4FU

worked SP1EB [Krotkofalewski Polski 6/1933] ??

EK4CV

EK4FW [E]

Dr. Constantin Wöllner (-1985), Siegen, DE1956H, DJ1QQ [E], [F]

Hans Rehder (- 1957), Hamburg Berlin-Zehlendorf, DE1946V, D3JAV, DL7BR [G]

EK4VX

Dr. Ing. Herbert Göttsching (->1995), Berlin, DE2179F, DL7CZ [G]

communicated with CV5BJ, 11/1931 [Krotkofalewski Polski 12/1931] ??

EK4VZ, station photo [TUC], heard by PL428, 11/1931 [Krotkofalewski Polski 12/1931][E]

heard overseas [CQ-MB 5/31] ??

communicated with CV5BJ, 11/1931 [Krotkofalewski Polski 12/1931] ??

heard overseas [CQ-MB 11/31] ??

EK4ME, EK4ABI

EK4RE

Friedrich Wilhelm Freund, Solingen Düsseldorf, DE1651H, D4BLH, D4ILH [G]

EK4NG 
Dr. Horst (Ernst) Podszus (? -1944 -1971[G]), Königsberg Hamburg, DE1319, D4BBA [E], D4CGA [CB1936], D4CGJ [CB1938][G][HH][OP]

D4DJE

D4DJJ

D4DJQ

D4DLD

D4DLI

D4DMS

D4DOB

D4DOC(2)

D4DPL

D4DRF

D4DSK

D4DUN

D4DVH

D4DWD

D4EAW

D4EDQ

D4EGM

D4EHR

D4EJB

D4EJT(2) [E]

D4ELK

D4ENF

D4EOW

D4EPF

D4ERH

D4ERI

D4ERM

D4ESE

D4ETB

D4EUH

D4EWR

D4EWV [E]

D4EWW

D4EXG

D4EZU

D4FAB

D4FBB

D4FBC

D4FBF

D4FBO

D4FBW

D4FCE

D4FCF

D4FCL

D4FCU

D4FDA(1)

D4FDA(2)

D4FDK

D4FEB

D4FEF

D4FFF(1)

D4FFF(2)

D4FFO

D4FFW

D4FGE [E]

D4FHF

D4FIB

D4FIR

D4FLA
EK4EJ

heard overseas [CQ-MB 10/31] ??

EK4QJ

EK4ME or other member of Funkvereinigung Halle/S KL4, EK4ABI [QSL from DE0489, TUC]

EK4IL

$\operatorname{EK} 4 \operatorname{SM}(1)$

communicated with SP1AT, 11/1931 [Krotkofalewski Polski 12/1931] ??

$\operatorname{EK} 4 \mathrm{CO}(1)$

EK4LP(1)

Robert Schmiedel (-1960), Berlin Münster, DE1586F, D4CQF, D4KKF, DL6QD [G][E]

EK4KS

EK4UN(1)

Johannes Willers (- 1993), Borna bei Leipzig Nürnberg, DE1643M, D4BSU, DC9NM, DL1NM [E], [G]

heard by PL198, 11/1931 [Krotkofalewski Polski 12/1931] ??

EK4WA(1)

Herbert Dancker (1916-1939 kia), Hannover Hamburg, DE1506K, D4BXK [E], D4BYJ, D4JXK,

prev call on D4BXK QSL unreadable [G]

EK4MG, communicated with SP3OL [Krotkofalewski Polski 12/1931][E][C]

EK4RG

EK4BJ

Rudolf Liefland (- 1954), Büdelsdorf Husum, DE1389V, D4BEV, D4JKV, DL3OP [E][CB1951-1954],[G]

Reinhard Liefland, Büdelsdorf, DL3OP [CB1957->1994], the son?

EK4KL

D4EWW

Karl Emde, Nürnberg Pfaffenhausen, DE1518P [E], D4EWV [E], HB9AY? [G]

EK4FP

signals heard by VK4GK [CQ-MB 2/31] ??

D4GQH, heard by PL423 [Krotkofalewski Polski 6/1933]

D4GQH

EK4ES, EK4EW

EK4BT

EK4UH

worked SP1EB [Krotkofalewski Polski 6/1933] ??

D4EOW, worked SP1BC between 4/1932 and 5/1933 [Krotkofalewski Polski 6/1933]

D4FPA

EK4GX

D4BTP

$\operatorname{EK} 4 \mathrm{BA}(1)$

$\mathrm{EK} 4 \mathrm{BB}(1)$

Heinz Böhnke (-1975), Luckenwalde, DE3348, EK4BP(2) in [G]?, DM2AND [E]

Hans Kaule, Berlin, DE1796F, D4ZAF [E], [G]

worked SP1EB [Krotkofalewski Polski 6/1933] ??

$\operatorname{EK} 4 \mathrm{NF}(2)$

EK4EC

Rudolf Köhler (- 1984), DE1811F, D4YBF, D4YGF, DL3DU [E], [G]

D4MVR

EK4UC [E], heard by PL423 [Krotkofalewski Polski 6/1933][V]

Gerhard Sackewitz (- 1993), Berlin Stuttgart, DE1352F, DL7BY [G]

Willi Happ, Berlin, DE1362, D4FFF(1) [G]

EK4KD

EK4BE

Willi Höpp, Berlin/Zehlendorf, DE1386F [TUC], [G]

D4FDA

Kurt Koschnieder, DE1356C/F, D4BXC, D4FFC [G]

EK4OF

EK4WF, heard by PL297, 11/1931 [Krotkofalewski Polski 12/1931]

EK4EB, EK4EG, 1930 QSL for QSO with G6YL [C]

Johannes Müller (- 1955), Berlin, DE1686F, DL7AM [G]

$\mathrm{EK} 4 \mathrm{KU}(2)$

worked SP1BC between 4/1932 and 5/1933 [Krotkofalewski Polski 6/1933] ??

Walter L. Baumgarten (1901- 1970), Berlin Genf Zandem, DE1396F, D4BLF suspended [CQ-MB 2/34], author in [CQ-MB 12/32, 7/33], emigrated to Switzerland, HB9SI (1956-1969), and Holland, PA0BB [G][K], in 1959 Chief of Communications, European Office of the United Nations, Geneva, operated in 1966 as 4U1SU (his 14th callsign) from 
D4FLN

D4FMJ

D4FML

D4FMP

D4FNX

D4FOE

D4FOX

D4FPA

D4FPB

D4FPF

D4FQF

D4FRG(1)

D4FRG(2)

D4FRQ

D4FSB [E]

D4FSD

D4FSG

D4FSI(1) [E]

D4FSJ

D4FTC

D4FTL

D4FTS

D4FVF

D4FWE

D4FXB

D4FXR [E]

D4FYB

D4FYE

D4FZN

D4GAG

D4GAI

D4GBA(1)

D4GBA(2)

D4GCF(1)

D4GCF(2)

D4GEA

D4GFX [E]

D4GGA

D4GGB

D4GGG

D4GGS

D4GHN

D4GHX

D4GIG

D4GIW

D4GJG

D4GJH [E]

D4GJM

D4GLD

D4GMF

D4GML

D4GNQ

D4GNW

D4GOQ

D4GOS

D4GPF

D4GQH
Gaza strip - radio interview with HB9GX: https://switzerlandinsound.com/vintage-mgrs/

heard by PL423 [Krotkofalewski Polski 6/1933] ??

EK4JM

EK4MH

EK4PM

EK4BX(1), EK4FN, EK4XN

$\operatorname{EK} 4 \mathrm{EO}(1,2)$

EK4UI, EK4XO

Heinz Windelband (1910-2001), Berlin Hamburg, DE1489F, D4FPA, D4EWW, D4ENF, D4CNF, D4HEF, DJ3UN, Op. at D4ADB [G][HH][OOTC1446], biography in [D], worked SP1EB [Krotkofalewski Polski 6/1933]

heard by PL428, 11/1931 [Krotkofalewski Polski 12/1931] ??

Johannes Bluhm (- 1993), Berlin, DE0061F, DE1961F, D4ZWF, DM3MO, Y24MO [G]

Karl Hirsch (- 1979), Berlin/Friedenau München, DE0317F, DE2178F, DJ3LL [E], [G]

Kurt Helfer (1910-2008), DE3957, D4VAS, DM3WJL, DM2DZL, Y24ZL, DL2DZL Dresden, started 1932 [OOTC2865]

Alois Chromy, Beuthen, O/S, D4BLG [E]

Franz Panniger, Weißenfels Münster Lager Landsberg, DE1681L/P [TUC, E], D4WUM(2), D4BJL 1935 [E],

D4MQL 1936 [E], D4FRF 1938, [G][E]

EK4BS

worked SP1EB [Krotkofalewski Polski 6/1933] ??

$1932[\mathrm{C}] ? ?$

EK4IS

EK4JS

EK4CT

Konrad Sieland (-1970), Eschwege Wahnfried/Werra, DE1547T, D4CHT(2), DL1CB [G][E]

YL operator, QSL 1932 [E] ??

Dr. Helmut(h) Buchmeier (- 1956), Beelitz Berlin Bad Driburg, DE1608C, D4NGA 4/1933, D4BDC, D4FVC, DL3VH

$[\mathrm{G}]$

EK4ES, EK4EW

$\operatorname{EK} 4 \mathrm{BX}(1)$

EK4RX [G]

heard overseas [CQ-MB 5/31]??

EK4EY [E]

Kurt Sperling, Zittau Tornesch, DE1114M [TUC], D4IRM, D4LCM, D4LPH, D4BYM, D4LPM [G]

EK4GA(1), communicated with SP1AH, 11/1931 [Krotkofalewski Polski 12/1931], heard by PL423

[Krotkofalewski Polski 6/1933][E]

$\operatorname{EK} 4 \mathrm{AI}(1)$

EK4AB

Bergedorf, Silesia, 1930 [E], 80m activity reported [CQ-MB 8/30, 11/30] ??

EK4CD

Hermann Halske (->1995), Lübeck, D4BPJ [E], D4KSJ [E], DL1JF, near Hamburg, 1932 QSL for QSO with G6YL [C] D4GCF heard by PL423 [Krotkofalewski Polski 6/1933]

EK4AE

EK4XF

80m activity reported [CQ-MB 8/30] ??

EK4GB(2)

Otto Mierzowski, Beuthen, Landshut, DE1134G [E][G], signals heard overseas [CQ-MB 7/31], communicated with SP3OL [Krotkofalewski Polski 12/1931], qso of "D4 dreimal Gustav" with D4DLD heard by DE1337A [TUC]

issued by Arbeiterradiobund ??

EK4NH

EK4XH

EK4GI

EK4CY, EK4WI

EK4GJ

EK4HJ(2)

Hans R. Sommer (-1981), Chemnitz Stettin Karl-Marx-Stadt, DE1498U [E], D4NFK, D4PQU [E], D4PQB [TUC], D4PQP [RL 1944], D4BVU?, DM2AEN [E] [CB1958], [G][E][DM6WAN]

Dr. Rudolf-Wilhelm Larenz, DE2388, Hannover Osnabrück Düesseldorf, D3BLK [E], DL3LJ [E], [G][E]

EK4FM

$\mathrm{EK} 4 \mathrm{HH}(2), \mathrm{EK} 4 \mathrm{ML}$

heard overseas [CQ-MB 11/30] ??

EK4AA, EK4WN, Mark Brandenburg, 1931 [E]

EK4QU(1)

Heinz Hollmach (- 1985), Leipzig, DE2120U, D3FFU, DM2ABM, Y21BM [J:8][G]

Paul Esser (-1991), DE1960I [TUC, E], D4GZF, D4ZZH, D4YRI, D4YZI, DL1WL [G]

Eberhard Kosche (-1938), Kiel Plauen Dresden, DE1631V, D4ERM, D4ERI 6/1933, D4BRU [E], D4FYF [CB1935], 


\section{D4GRW}

D4BGV [G]

D4GSG

Georg Schober (- 1968), Berlin Seeheim, DE1355F, Op at D4ADH, DL3DQ(2) [G]

Fritz Rother (-1974), Staatl. Pol. Schule Frankenstein/Schlesien, D4TEG, D4BDG [E], D4HPG, DL1QA [E], 1932 [E][V], heard by PL297, 11/1931

[Krotkofalewski Polski 12/1931]

D4GTW Maria Tama, Berlin Barcelona, DE1692X/F [G]

D4HAG(1)

D4HAG(2)

D4HBT

EK4GC [E]

D4HEC(1)

Germany South-East 1932 [E] ??

Wilhelm Schäfer (-2003), Backnang, DE2449 [E], D4WBT, D3JGF, DA1HI, DL1LZ [E], [G]

D4HEC(2)

Normann von Derstein, Köln/Nippes, DE1527I [G]

D4HIK

D4HJT

D4HMO

D4HNR

D4HOB

D4HOL

D4HOV

D4HVA

D4HWB

D4HZL

D4IAH

D4CAR

Karl (Konrad) Hillenbrand (- 1967), Frankfurt a.M., DE1701X, D4OPT [KFL1944], D4BMT [E], DL1BY [G]

EK4TJ

D4OMO

D4IDF

Hans Nischkowski, Reichenbach, DE1229G [G]

heard overseas [CQ-MB 11/31] ??

EK4DZ

Guttentag, Berlin Königsberg, DE1453A [G]

EK4BW(2)

Ing. Georg Müller, Bamberg, DE2040R [G]

EK4AH(1), EK4SAR

worked SP1BC between 4/1932 and 5/1933 [Krotkofalewski Polski 6/1933]

EK4RC 1931 [E]

D4ICR

D4IDA

D4IDF

D4IDU

heard by PL423 [Krotkofalewski Polski 6/1933] ??

Ernst Fendler (-1987), Berlin Duisburg Adenstedt Pforzheim, DE1576H, D4HNR, D4BAH?, D4BBH, D4IDH, DA3JU, DA4JV, DL1JK [G]

EK4UD, heard by PL198, 11/1931 [Krotkofalewski Polski 12/1931]

$\operatorname{D} 4 \operatorname{IFF}(1)[\mathrm{E}]$

D4IFG

D4IHA

D4IHB

D4IIG

D4IJN

D4ILU

D4IMI

D4IND

D4INI

D4INN

D4IPF [E]

D4IRG

D4IRM

Richard Hack, Hamburg, DE1624J, D4BLJ [E], D4KOJ, DJ1AK [E], [G][DJ1GE]

heard overseas [CQ-MB 11/30] ??

EK4AH

EK4BH(1)

Gerhard Ouvrier, Gleiwitz Dornhausen, DE1392G, D4CCG, D4SBG [G]

signals heard overseas [CQ-MB 3/31] ??

heard by PL428, 11/1931 [Krotkofalewski Polski 12/1931] ??

EK4IM(2), 1931 [E], heard by PL423 [Krotkofalewski Polski 6/1933]

EK4HZ

EK4HZ

EK4NN

Franz von der Hude (- 1955), Lübeck Neumünster Kiel Rendsburg List Sylt, DE1559J, D4BEJ, D4JIJ, DL1YS [V][E]

signals heard overseas [CQ-MB 3/31], communicated with CV5BJ, 11/1931 [Krotkofalewski Polski 12/1931] ??

D4ISN

D4ITD

D4ITE

D4ITF

D4IWF

D4JAV

D4JFG

D4JGF

D4JHV

D4JJU [E]

D4JMM

D4JNW

D4JPC.[E]

D4FZN

D4LLL

EK4TD

Erich Struß (-1966), Allenstein Lykusen, DE1714J, D4BDA, D4AYA [G][OP], poem in [CQ-MB 10/32]

EK4FT(1)

EK4FW

EK4PL(3)

Werner Weihe, Liegnitz, DE1610G, QSL 1935[V][C], [G]

Ing. Horst Günther (->1995), Berlin Harburg Kiel, DE1582F/N, D4BIJ [E], D4JGV, DJ6UC [CB1962] [G]

D4BHV

EK4UJ, worked SP1BC between 4/1932 and 5/1933 [Krotkofalewski Polski 6/1933]

EK4MM

Eduard Jäger (-1966), Hohenems, DE1266W, UOWM, UO7EJ, OE9EJ, OE3EJ, OE1EJ,

D4JRG

D4JUR

D4JXM

D4KAI

D4KAN

D4KBR

D4KBV

EK4CP [E]

EK4GL [E]

EK4RY, EK4JU

station photo [TUC] ??

EK4HZ

Arthur M. Braaten, Long Island, DE1563, NU9EFW, W2BSR [C], DL3OR? [G]

Kurt Breutzmann, Köln, D1514I [E], D4BLI, D4IYI [E], [G]

$\operatorname{EK} 4 \mathrm{VB}(1)$

D4KEY

Dr. Hanns Albrecht G. Hess (1910-1991), Ulm, DE1603N, D4DHX, D4BKN [E], D4MDN[E], [G][V],

[Phys. Bl. 1985 41/5, A-210, Phys. Bl. 1990 46/12, 457], physicist, researched propagation of shortwaves with Karl Rawer - author [Hess, 1946, 1947, Hess \& Rawer, 1960] 
D4KHM

D4KIP

D4KKK

D4KLA

D4KLG

D4KMG

D4KOE

D4KOI

D4KPJ

D4KQM

D4KRO

$\mathrm{D} 4 \mathrm{KSH}$

D4KTM

D4KTP

D4KVM

D4KZG

D4KZN

D4KZR

D4LAH

D4LAL [E]

D4LAN

D4LBJ

D4LBM

D4LBT

D4LCH

D4LCJ

D4LCM

D4LDX

D4LEM

D4LFQ

D4LFU

D4LGB(1)

D4LGB(2)

D4LGH [E]

D4LGM

D4LGR [E]

D4LGS

D4LGX

D4LKA [E]

D4LKH

D4LKJ

D4LKU

D4LLJ(1)

D4LLL(1)

D4LLL(2)

D4LLW

D4LMA

D4LMH [E]

D4LMT

D4LMW

D4LNH(1)

D4LNH(2)

D4LNK

D4LOD

D4LOP [E]

D4LPH

D4LPM
Otto Borkenhagen (-1981), Maulbronn Dresden Wellingsdorf Calvins Kiel, DE2357V, D4TEV, DA1CG, DL1CM, DF3LE $[\mathrm{G}]$

\section{EK4PI}

Karl Gerlinger, Kassel, DE1617K [G], heard by PL423 [Krotkofalewski Polski 6/1933]

EK4AL(1), heard by PL423 [Krotkofalewski Polski 6/1933]

EK4GL

EK4GM

EK4IC(1), EK4IA, EK4EO

EK4IK

EK4JP

Erich Kartheuser (- 1956), Dresden Nürnberg, DE1534M, D4KVM, D4BXM, D4LOM, DL3GD [G]

EK4RR(1), EK4RO

Ing. Heinz Schicktanz, DE1414, Sohland/Spree near Bautzen, D4BOM [E], D4LIM [G]

EK4MT(1)

EK4PT

D4KQM

D4BZG

D4BXT

Ferdinand G. Tillmanns (-1950), Köln, DE1540I, D4BXI, D4YMI [G][E]

worked SP1EB [Krotkofalewski Polski 6/1933] ??

EK4LA [E], worked SP1BC between 4/1932 and 5/1933 [Krotkofalewski Polski 6/1933]

Ing. Kurt Bretschneider (-1957), Leipzig, Limbach/Sachsen Wahnfried, DE1546U, D4BLU [E], D4PIU [E], DA3RA, [G][DM6WAN]

Willi Schweer (- 1975), Hamburg-Altengamme, DE2162J, DL1SA [G]

EK4MB

EK4IB, EK4WO

heard by PL198, 11/1931 [Krotkofalewski Polski 12/1931] ??

Hans Schlottmann (- 1984), Berlin Marne/Holstein Friedrichshafen, DE1783V, D4CTF, D4CTV, DL1UL [CB1949-1981], DL3ZY?, [G]

D4FZN

Helmut K. E. Schall (->1992), Stuttgart Neresheim, started 1929, D4LDN, D4BDN [CB1934], D4LUN [CB1935],

DL1LY [E], worked SP1EB [Krotkofalewski Polski 6/1933]

EK4MX

Gerhard Fritze, Magdeburg, DE1374D [E], D4BND, Op at D4ADZ, D4BAD suspended [CQ-MB 2/34][G]

EK4UF(1)

EK4GB(2)

Gotthold Danzke (- 1958), Landsberg/Halle Eschwege, DE1635L [TUC], D4BDL, D4MNL, D4MNU,

DL6QV [CB1952-1958]

\section{EK4HG}

Ing. Georg Zumpe (1912-2010), Seifersdorf/Sachsen Chemnitz, DE1535M, D4LZH, D4BTM, D4LKM [G][DM6WAN] $1930 ? ?$

EK4GS

EK4XY, EK4XG

1932 ??

EK4HK

EK4JK

D4MPR [E]

EK4JL(1), D4BOK [E], signals heard overseas [CQ-MB 7/31]

Anton Esser (- 1961), Ludwigshafen Essen Wesseling Köln Friedrichshafen, DE1311T, D4ISN, D4NIL, D4BEI, D4IST, D4ISH, DL1MW [G]

Günter Zobel (-1974), Blankenburg am Harz Villingen Ulm, DE1556D [E], DE6658, D4LLL (1930-1932),

D4NZP (1932-33), D4BOS, D4MZQ, D4VIK, DJ4DN [G], D4NZP QSL of 1933 from nr Magdeburg [C], call info DJ4DN QSL of 1958 [E]

heard overseas [CQ-MB 8/31] ??

EK4AM

1930 ??

EK4TM

EK4WM(1)

EK4HN

$\mathrm{D} 4 \mathrm{MOM}(2)$

EK4KG(1)

EK4PT

EK4PO, heard overseas [CQ-MB 9/31]

D4FZN

EK4MK 
D4LPP

D4LQH [E]

D4LQT [E]

D4LRJ

D4LRM [E]

D4LRP [E]

D4LRR

D4LRX

D4LUH

D4LUP

D4LWL

D4LWR

D4LXH [E]

D4LYH

D4LYR

D4LYT

D4LZH

D4MAQ

D4MBP

D4MGC

D4MEH

D4MEL

D4MEV

D4MEW [E]

D4MFM [E]

D4MFQ

D4MGR

D4MHM

D4MHW [E]

D4MIM

D4MIR [E]

D4MKG

D4MKL

D4MLD

D4MLH

D4MLQ

D4MLW

D4MMX

D4MOF

D4MOM(1)

D4MOM(2)

D4MOP

D4MOR

D4MOW [E]

D4MPB [E]

D4MPL

D4MPR [E]

D4MRJ

D4MRO [E]

D4MSG [E]

D4MUR

D4MUZ

D4MVF [E]

D4MVK

D4MVR

D4MWH

D4MYS

D4MZF

D4NAH

D4NAI [E]

D4NAN
EK4DP, heard by PL198, 11/1931 [Krotkofalewski Polski 12/1931]

EK4MW, Sachsen, 1933

EK4TQ(1), communicated with SP1AH, 11/1931 [Krotkofalewski Polski 12/1931]

EK4DP

EK4HI, EK4MR, communicated with SP3OL [Krotkofalewski Polski 12/1931]

1931 ??

EK4RR(2)

Georg Lotze (- 1990), Mannheim Clausthal Hannover, DE1832O, DL9JY [G]

EK4HU

QSL from D4BRJ 1933 [E] ??

Martin Schädelbach (-1985), Dresden Eisenach Berlin München, DE1564L, D4BTI, D4BVM, D4LMM, D4LMF, DL7BO [CB1952-63][E], DF2MG [CB1981] [G]

Harri Worm (-1958), Köln/Riehl, DE1654I [TUC], D4BMI [E], D4IZI [E], [G]

Erich Fehrmann, Chemnitz Berlin, DE1385U, D4PNM, D4BPM, D4PNU, D3PNF [QSL 1931, [G] [DM6WAN]

EK4HY(2)

EK4RY, EK4JU, heard by DE1516 [QSL to D4ABI, TUC]

EK4TY

D4LGM

EK4AQ

EK4BK(1)

$\mathrm{EK} 4 \mathrm{CG}$

D4BVL

EK4WL(1)

communicated with CV5BJ, 11/1931 [Krotkofalewski Polski 12/1931] ??

Heinz Lange (- 1985), Berlin, DE1354F, D4FJF, DL7CL [G][E], communicated with SP3OL

[Krotkofalewski Polski 12/1931], heard by PL423 [Krotkofalewski Polski 6/1933][E]

EK4MF

EK4QF

heard by PL428, 11/1931 [Krotkofalewski Polski 12/1931] ??

worked SP1BC between 4/1932 and 5/1933 [Krotkofalewski Polski 6/1933] ??

EK4WH [E], worked SP1AT before 4/1933 [Krotkofalewski Polski 6/1933]

signals heard overseas [CQ-MB 2/31] ??

1933, LGL5 [C] ??

EK4GK

EK4LC

EK4BU(2) [E]

EK4HL(1)

EK4QL

communicated with SP1AT, 11/1931 [Krotkofalewski Polski 12/1931] ??

EK4XM

EK4IB, EK4WO

EK4MO

Heinz Kluge (-1971), DE1406, Cuxhaven Wilhelmshaven, D4LNH(2), D4BDG, DL6IE [G]

EK4PO

Helmut Fehlemann (-1972), Duisburg, DE1160H, D4LGK, D4LGH, DL6JT [G]

EK4IB, EK4WO

EK4BP(1), EK4MA claimed in [G][U] - but writing looks different as on D4MA qsl, ??

D4BWH(1)

Johannes Kotthoff (-1979), DE1307H, D4BIR [E], D4BTH, D4LKU, D3DAH?, D3DAP, DL3RM [G]

communicated with SP3OL [Krotkofalewski Polski 12/1931]

Rudolph (Rudi) Meissner (1913-1998), Hameln Uelzen, DE1693K, started 1932, EZ4BB, DA5OM, DA5YM, DL1QP [E], [OOTC2587][E]

$1931[\mathrm{C}] ?$ ?

EK4GU, communicated with CV5BJ, 11/1931 [Krotkofalewski Polski 12/1931]

EK4RU(1)

heard by PL297, 11/1931 [Krotkofalewski Polski 12/1931] ??

1933 Hamburg ??

Walter Vrancken, Frankfurt/Main, DE1767T, D4BIT(2), D4OLT [G]

Peter Tolles (- 1957), bei Krefeld, DE1306, D4FCL, D4BGH(3), D4KGH, DL1JL, Haushälterin Frieda Blum, DE1954 [E]

EK4HW(1)

EK4SZ, EK4SY

Hans-Heinrich Heider (-1970), Hamburg Cuxhaven, DE1660J, D4BIB, DL1IN [G]

heard by CE7AA [CQ-MB 3/31]

EK4IC(1), EK4IA, EK4EO [E], station photo [TUC], signals heard overseas [CQ-MB 3/31]

EK4NA 


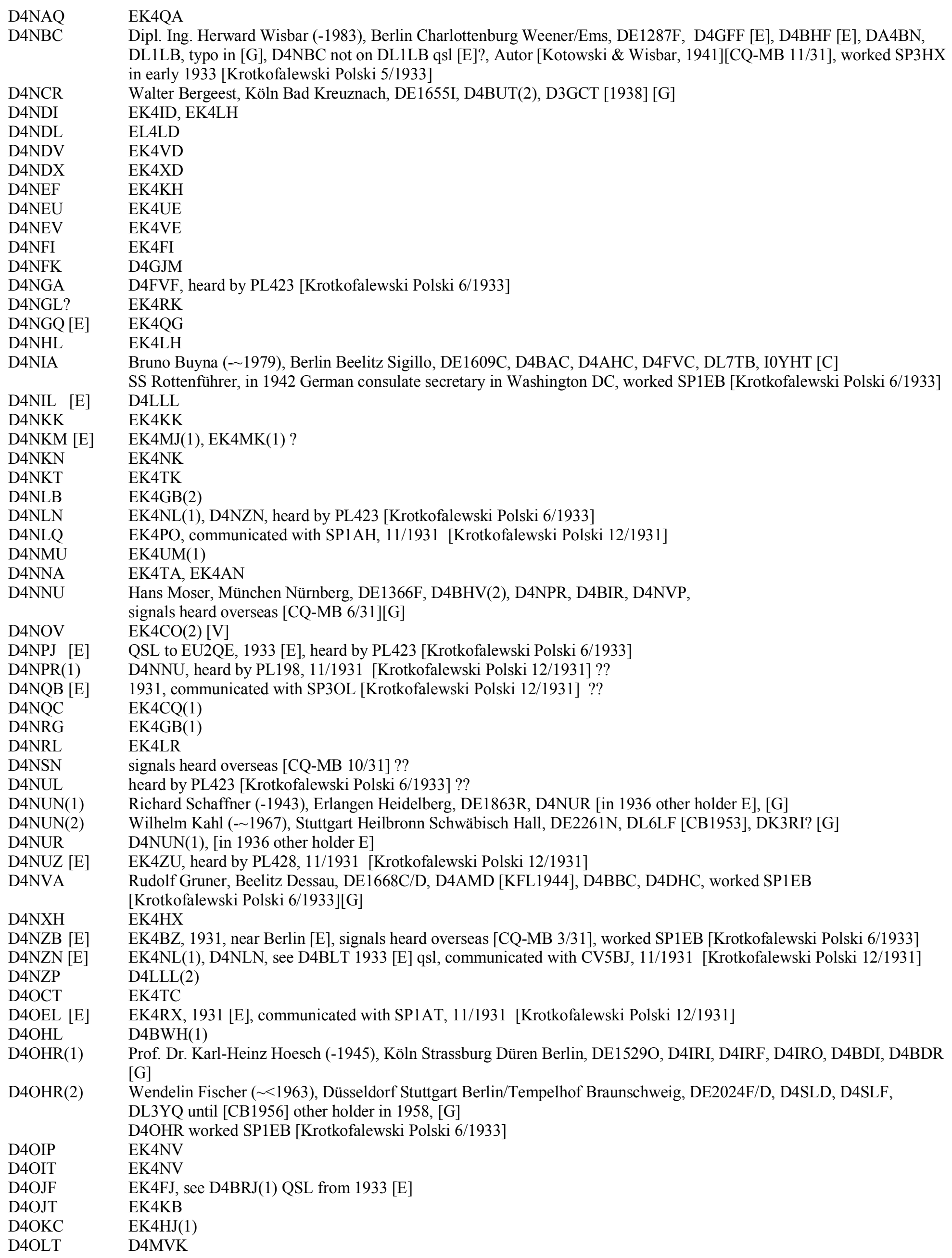

D4NAQ

D4NBC

D4NCR

D4NDI

D4NDL

D4NDV

D4NDX

D4NEF

D4NEU

D4NEV

D4NFI

D4NFK

D4NGA

D4NGL?

D4NGQ [E]

D4NHL

D4NIA

D4NIL [E]

D4NKK

D4NKM [E]

D4NKN

D4NKT

D4NLB

D4NLN

D4NLQ

D4NMU

D4NNA

D4NNU

D4NOV

D4NPJ [E]

D4NPR(1)

D4NQB [E]

D4NQC

D4NRG

D4NRL

D4NSN

D4NUL

D4NUN(1)

D4NUN(2)

D4NUR

D4NUZ [E]

D4NVA

D4NXH

D4NZB [E]

D4NZN [E]

D4NZP

D4OCT

D4OEL [E]

D4OHL

D4OHR(1)

$\operatorname{D4OHR}(2)$

D4OIP

D4OIT

D4OJF

D4OJT

D4OKC

D4OLT

EK4QA

Dipl. Ing. Herward Wisbar (-1983), Berlin Charlottenburg Weener/Ems, DE1287F, D4GFF [E], D4BHF [E], DA4BN, DL1LB, typo in [G], D4NBC not on DL1LB qs1 [E]?, Autor [Kotowski \& Wisbar, 1941][CQ-MB 11/31], worked SP3HX in early 1933 [Krotkofalewski Polski 5/1933]

Walter Bergeest, Köln Bad Kreuznach, DE1655I, D4BUT(2), D3GCT [1938] [G]

EK4ID, EK4LH

EL4LD

EK4VD

EK4XD

EK4KH

EK4UE

EK4VE

EK4FI

D4GJM

D4FVF, heard by PL423 [Krotkofalewski Polski 6/1933]

EK4RK

EK4QG

EK4LH

Bruno Buyna (- 1979), Berlin Beelitz Sigillo, DE1609C, D4BAC, D4AHC, D4FVC, DL7TB, I0YHT [C]

SS Rottenführer, in 1942 German consulate secretary in Washington DC, worked SP1EB [Krotkofalewski Polski 6/1933]

D4LLL

EK4KK

EK4MJ(1), EK4MK(1)?

EK4NK

EK4TK

$\operatorname{EK} 4 \mathrm{~GB}(2)$

EK4NL(1), D4NZN, heard by PL423 [Krotkofalewski Polski 6/1933]

EK4PO, communicated with SP1AH, 11/1931 [Krotkofalewski Polski 12/1931]

$\operatorname{EK} 4 \mathrm{UM}(1)$

EK4TA, EK4AN

Hans Moser, München Nürnberg, DE1366F, D4BHV(2), D4NPR, D4BIR, D4NVP, signals heard overseas [CQ-MB 6/31][G]

$\mathrm{EK} 4 \mathrm{CO}(2)[\mathrm{V}]$

QSL to EU2QE, 1933 [E], heard by PL423 [Krotkofalewski Polski 6/1933]

D4NNU, heard by PL198, 11/1931 [Krotkofalewski Polski 12/1931] ??

1931, communicated with SP3OL [Krotkofalewski Polski 12/1931] ??

EK4CQ(1)

$\mathrm{EK} 4 \mathrm{~GB}(1)$

EK4LR

signals heard overseas [CQ-MB 10/31] ??

heard by PL423 [Krotkofalewski Polski 6/1933] ??

Richard Schaffner (-1943), Erlangen Heidelberg, DE1863R, D4NUR [in 1936 other holder E], [G]

Wilhelm Kahl (- 1967), Stuttgart Heilbronn Schwäbisch Hall, DE2261N, DL6LF [CB1953], DK3RI? [G]

D4NUN(1), [in 1936 other holder E]

EK4ZU, heard by PL428, 11/1931 [Krotkofalewski Polski 12/1931]

Rudolf Gruner, Beelitz Dessau, DE1668C/D, D4AMD [KFL1944], D4BBC, D4DHC, worked SP1EB

[Krotkofalewski Polski 6/1933][G]

EK4HX

EK4BZ, 1931, near Berlin [E], signals heard overseas [CQ-MB 3/31], worked SP1EB [Krotkofalewski Polski 6/1933]

EK4NL(1), D4NLN, see D4BLT 1933 [E] qsl, communicated with CV5BJ, 11/1931 [Krotkofalewski Polski 12/1931]

D4LLL(2)

EK4TC

EK4RX, 1931 [E], communicated with SP1AT, 11/1931 [Krotkofalewski Polski 12/1931]

D4BWH(1)

Prof. Dr. Karl-Heinz Hoesch (-1945), Köln Strassburg Düren Berlin, DE1529O, D4IRI, D4IRF, D4IRO, D4BDI, D4BDR [G]

Wendelin Fischer ( <1963), Düsseldorf Stuttgart Berlin/Tempelhof Braunschweig, DE2024F/D, D4SLD, D4SLF,

DL3YQ until [CB1956] other holder in 1958, [G]

D4OHR worked SP1EB [Krotkofalewski Polski 6/1933]

EK4NV

EK4NV

EK4FJ, see D4BRJ(1) QSL from 1933 [E]

EK4KB

EK4HJ(1)

D4MVK 
D4OLU

D4OMG

D4OMF

D4OMK

D4OMO

D4ONE

D4OOB

D4OOF

D4000(1)

D4000(2)

D4OON

D4OPG [E]

D4OPQ

D4ORQ

D4OSA [E]

D4OSO

D4OVT

D4OWE

D4OYG [E]

D4OZX

D4PAB [E]

D4PAD

D4PDC

D4PGG

D4PGW

D4PHD

D4PHU

D4PJL

D4PKU(1)

D4PKW [E]

D4PLL

D4PMK

D4PMV

D4POB

D4POC

D4PSG [E]

D4POJ [E]

D4POL

D4PSO [E]

D4PSQ

D4PUG

D4PUO [E]

D4PUQ(1)

D4PUQ(2)

D4PUR

D4PVQ

D4PVT

D4PWE [E]

D4PWG

D4PWW [E]

D4QDS

D4QRE

D4QVT

D4QWW

D4RAA

D4RAC

D4RAD

D4RAU

D4RAZ [E]
EK4UL(3)

Bernhard Puschmann (- 1980), 1931, Düsseldorf Beinberg/Black Forest, DE1398, D4HWG, D4BJG, DA4BU, DL1JJ [G], author [Puschmann, 1962][Puschmann \& Koerner, 1969]

Wilhelm Wolf, Falkenstein Berlin, DE1765T, D4OMK, D4WWS, D4BJT [G]

D4OMF

Adolf K. Freiherr von Supper (1899- 1970), Karlsruhe, DE1467O [TUC, E], GOETZ, D4HMO, D4BBO [E], D4NBO, D4NOB, DA5AR, DL1EZ [CB1957-1972], worked in the city administration of Karlsruhe, SS-Hauptsturmführer member since 1929, NSDAP since 1930, was recruited by the CIA in 1954, operated under pseudonym "Holzer"

[Schmitt-Enboom \& Stoll, 2015][Lecorte 2015][G], bio in [CQ-MB 7/1937]

EK4EN, worked SP1BC between 4/1932 and 5/1933 [Krotkofalewski Polski 6/1933]

EK4NL(1)

$\mathrm{EK} 4 \mathrm{FO}$

EK4NL(1)

Erich Oppermann (->1995), Hannoversch-Münden, DE1815D, D4BUK, D4GAD, DL3GA [G]

EK4NL(1)

EK4GP, communicated with SP1AH, 11/1931 [Krotkofalewski Polski 12/1931][E]

heard by PL423 [Krotkofalewski Polski 6/1933] ??

EK4QR

EK4AS

communicated with SP3OL [Krotkofalewski Polski 12/1931] ??

Heinz Finhold, Bad Kreuznach, DE1241 [E], D4NQN, D4CCT, D4BCQ [CB1934],[G]

EK4EW(2)

EK4DZ, EK4GY

heard by PL198, 11/1931 [Krotkofalewski Polski 12/1931] ??

Germany (East), 1930 ??

signals heard overseas [CQ-MB 3/31] ??

EK4CD

EK4OW(2)

EK4GW

EK4AH(2)

Erich Götze, Plauen, DE0749 [G]

EK4LJ

EK4UK

EK4WK(1), communicated with SP3OL [Krotkofalewski Polski 12/1931]

EK4PT, EK4LL

D4BTT

EK4VZ

Herbert Wohlan (- 1970), Stettin, DE1801C/B, DL1ZX [G]

D4POB

near Ratibor Silesia, 1931 [E] ??

EK4JO, worked SP1EB [Krotkofalewski Polski 6/1933]

EK4PA, EK4OL(2)

$\mathrm{EK} 4 \mathrm{HH}(1)$

Hermann Siems (-1937), DE0359, D4CMA, Op at EK4AEY, built x-tal driven 5m transmitter in 1931, contact with SP3OL 10/1931 [Krotkofalewski Polski 12/1931], was suspended from the club because of lack of interest, drowned in the north sea $[\mathrm{G}]$

1931 [C] ??

1931 ??

EK4QU(2)

EK4UQ

EK4RU(1)

EK4QV, EK4AN(1)

EK4PV

EK4ES, EK4EW $(1,2)$

EK4OW(2)

EK4WW?, EK4QY?, (similar hand writing) Hannover Land

1932, [C] ?? Arbeiterradiobund?

EK4TT, communicated with SP3OL [Krotkofalewski Polski 12/1931]

EK4TE(1), EK4TV(2)

EK4QY(1), EK4WW

EK4AA

EK4CA

1929, QSL to D4DLD [TUC] ??

EK4UA(1)

Bayern 1931 [C], 1932 [V], activity reported [CQ-MB 11/30], heard by PL428, 11/1931 [Krotkofalewski Polski 12/1931], 
D4RBD

D4RCA

D4RCC

D4RCH

D4RDG

D4RDP [E]

D4RDW [E]

D4RDX

D4REF

D4REP

D4REZ

D4RFP [E]

D4RGH

D4RGU [E]

D4RGW

D4RHA

D4RHH

D4RHL [E]

D4RHR(1)

D4RHR(2)

D4RIB

D4RIM

D4RIX

D4RJA

D4RJR

D4RJW

D4RKA

D4RKN [E]

D4RKP [E]

D4RKV

D4RLM [E]

D4RLN

D4RMB [E]

D4RML

D4RNG [E]

D4RNX

D4RPI

D4RPM

D4RPN

D4RPU

D4RPV

D4RQL

D4RRC [E]

D4RRF

D4RSJ

D4RSR

D4RSU

D4RSV [E]

D4RTA(1)

D4RTA(2)

D4RUH

D4RUK

D4RUQ

D4RUS

D4RUT [E]

D4RUX

D4RVJ(1)

D4RVM worked SP1EB [Krotkofalewski Polski 6/1933][E][V] ??

D4RDP

$\mathrm{EK} 4 \mathrm{AC}(2)$

EK4CC

Cologne, 1930, [C] ??

EK4GD(1), communicated with CV5BJ, 11/1931 [Krotkofalewski Polski 12/1931]

Ernst Bußmann (-1964), Gardelegen (Altmark), Dresden, DE1345M, D4RBD, D4BDD [E], D4BBM, D4LDM [E], [G] communicated with SP1AT, 11/1931

[Krotkofalewski Polski 12/1931], heard by PL423 [Krotkofalewski Polski 6/1933]

EK4WD?, near Berlin 1931 [E], signals heard overseas [CQ-MB 3/31] ??

Prof. Dr. Alfred Lotze (1915-1989), Stuttgart, DE1504N, D4BFN, D4LWN [E], DL1HQ, later Director of the Institute for

Switching and Data Techniques at the University of Stuttgart [G], communicated with SP1AT, 11/1931

[Krotkofalewski Polski 12/1931]

Rolf Tschander, DE1672J, Hamburg, D4BBJ [CB1934], D4KEJ [G]

communicated with SP1AH, 11/1931 [Krotkofalewski Polski 12/1931] ??

EK4UAG(2)

Herbert Fehse, Gardelegen, DE1344D, D4BCD [E], [G] heard by PL428, 11/1931

[Krotkofalewski Polski 12/1931], heard by PL423 [Krotkofalewski Polski 6/1933]

D4CFT

1932 ??

Georg Paffrath (-1970), Berlin Linz/Rhein, DE1260F, D4XOF, D4BFH, DL6EG [G]

worked SP1EB [Krotkofalewski Polski 6/1933] ??

qso with VE1DR on 3/1931 [CQ-MB 11/1935]

1931 [E], worked SP1EB [Krotkofalewski Polski 6/1933] ??

Hans Bodo Asbach, Köln, DE1531I, D4BCI, D4IQI [CB1936]

EK4RG, Werner Hess received WAC [QST 03/1933]

D4RHR signal received overseas [CQ-MB 7/31, 1/32][QST 3/1933]

EK4BI(1)

EK4MI

EK4XI

EK4JA

EK4RJ

EK4WJ

Edgar Rosenbaum, DE1402 [E], Berlin [G]

$1932 ? ?$

EK4KP, communicated with SP1AT, 11/1931 [Krotkofalewski Polski 12/1931]

$\operatorname{EK} 4 \mathrm{VK}(1)$

1932, station photo [TUC], ??

heard by PL428, 11/1931 [Krotkofalewski Polski 12/1931] ??

EK4BM(1)

EK4ML

near Breslau 1930 [C][V], signals heard overseas [CQ-MB 6/31] ??

Ernst Walter (- 1985), Hamburg, DE2151J, DE2161J, D4AJJ, DA6MI, DL1SC [E], [G]

EK4IP

EK4HL(1) [V]

signals heard overseas [CQ-MB 6/31]??

EK4PU

Karl Tetzner (->1995), Leipzig Emden München Hamburg, DE1774M, DL1UH [G]

EK4LQ

EK4CR

EK4FR

Hans Rhode, Cuxhaven Sprengel, DE1921V [G]

communicated with CV5BJ, 11/1931 [Krotkofalewski Polski 12/1931]??

EK4US

maybe EK4VS(1), 1930, communicated with SP3OL, 11/1931 [Krotkofalewski Polski 12/1931][E] ??

EK4TP

Georg Viehweger, Berlin Kiel, DE1295F [G]

Paul Engelhardt, Recklinghausen, DE1528H, D4CDH [G]

EK4KU(1)

worked SP1BC between 4/1932 and 5/1933 [Krotkofalewski Polski 6/1933] ??

EK4US

EK4TU, communicated with SP1AT, 11/1931 [Krotkofalewski Polski 12/1931][E]

EK4XU(2)

$\operatorname{EK} 4 \mathrm{VJ}(1)$

EK4HC 


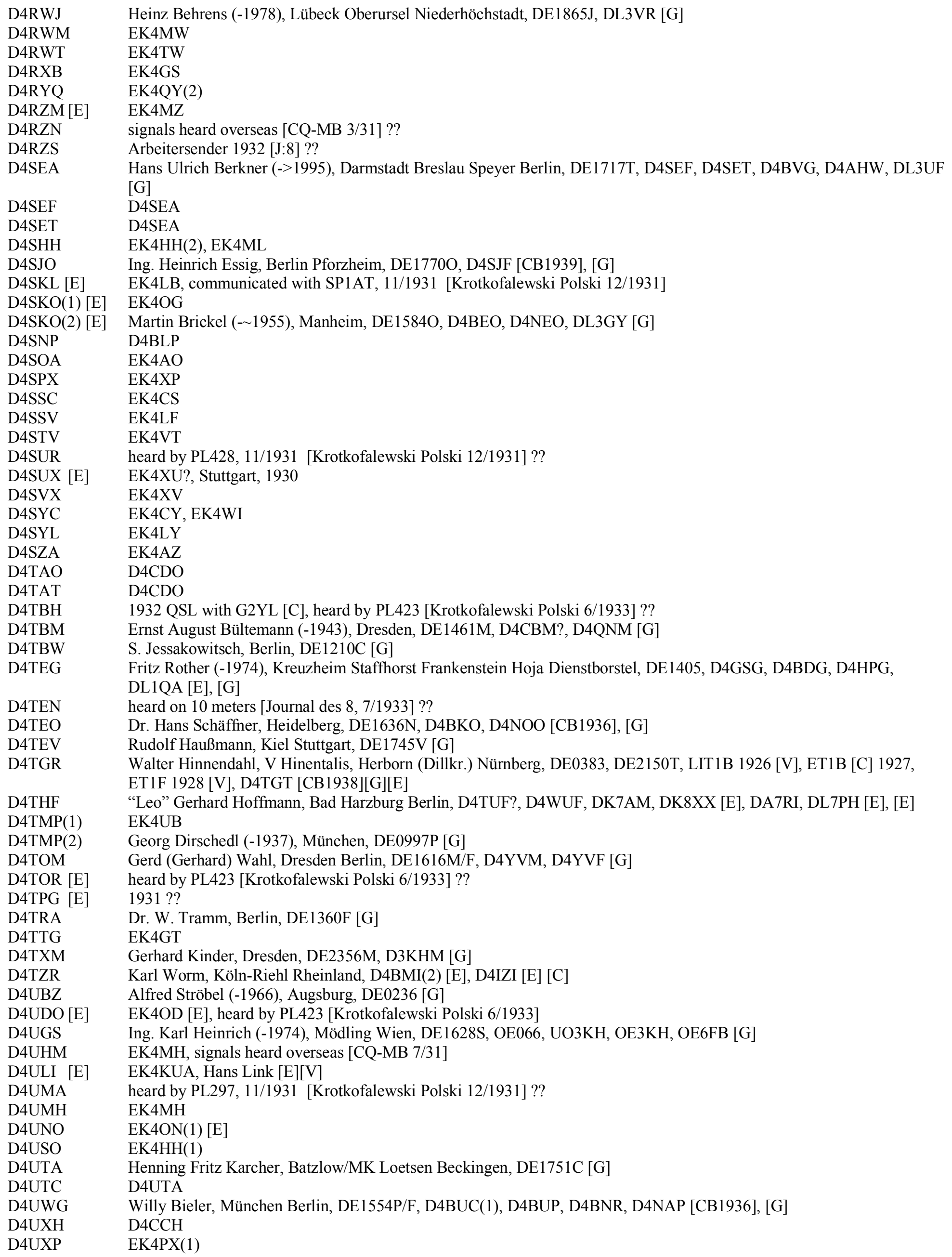







worked SP1BC between 4/1932 and 5/1933 [Krotkofalewski Polski 6/1933]

D4ZBP[CB1938] Ernst Aschbacher, München, DE0735P [C], DE1974F [MB-HVL 10/32]

D4ZGM[CB1938] Prof. Friedrich Siegert (-1960ies), Dresden, Sickingenstr. 1, DE0739M [C], DE2770, author in [CQ-MB 1/30] headmaster and teacher at Dreikönigsschule Dresden, Sickingenstr. 1. Emigrated in 1946 with his wife Lea, passed away in the late 1960ies in New York, USA [Gedenkbuch der Jüdischen Gemeinde zu Dresden][G]

D4ZNB EK4BN

D4ZOL Karl H.-Hamer (-> 1995), Dortmund-Hörde, DE1557H, D4BPH [E], D4INH, DL6RH [G]

D4ZQW [E] Ernst Nickel (- 1985), Hamburg Berlin/Pankow Heilbronn, DE1353F, D4CMF, D4CFJ, D4HDF, DJ3VR [G]

D4ZUA EK4AU

D4ZUG signals heard overseas [CQ-MB 3/31] ??

D4ZUL [E] 1931 ?? 
2c) Foreign calls 1929-1932 of DE holders, EK and D stations, further unlis operations:

CT2AB [E] Max Corsepius (1893-1968), Dresden Köln Horta Fayal Azores Frankfurt Eschborn Oberursel, DE1341, DJ4UN [G][E] Born in Dresden, where his father Max was docent at Technische Hochschule Dresden [Corsepius 1891], the family moved later to Köln. The father died in 1910 and at the age of 17 he took up a job with the Deutsch-Atlantische Telegraphengesellschaft (DAT) at the submarine cable station in Horta Azores. In 1916 he was detained as a prisoner of war on Terceira Island until the end of WWI. In 1926 he returned to the Horta location of DAT and became the first Technical engineer. He is listed as CT2AB in [CB1932-1939] and became very active on the air in the years 1936-1939. In May 1939 he went to Germany for vacation and was denied return to Portugal because of WWII. He worked for the German Post Office during the war years, and transferred in 1944 to Plau Mecklenburg. In 1948 he moved to West Germany [Corsepius 2013]. He got relicensed as DJ4UN before his retirement in 1960 [E].

CV5AF $[\mathrm{C}]$

CV5AV [C]

HB9AG

HB9N

OK2AK

OK2OP [E]

OK2RM[E]

PA0CM [CB38]

RY1A [C]

RY1AA

RY1E [C]

RY1T

RY1X [C]

SP1AD [CB32]

TS4SAA

TS4SAC(1)

TS4SAC(2)

TS4SAX

TS4SAZ [E]

TS4SBR

TS4SKL

TS4SR

TS4SRC

TS4SUP

TS4SUR

TS4SZ

UN1XZ

UN7DD

UN7GL [E]

UN7LL

UN7PP [E]

UN7SJ

UN7VV [E]

UOAA

UOBG

UOCK

UOCX

UOJZ

UOKL

UOSK

UOSL

UOWG

UOWN [E]

UOXR

UO1AR

UO1CM

UO1FH

UO1JZ

UO1SL

UO1WG

UO3F

$\mathrm{UO} 3 \mathrm{HU}$

UO3WB
Carl Bratescu, Romania, DE0893, ER5AF, YO7AG [C],[G]

Valentin Calinescu, Targoviste Romania, DE0568, YR5IJ [C],[G]

Dr. Erwin Huber, Zürich Bern, DE1509, CT1XU, [G]

Werner Niederer, Zürich Berlin, DE0868, HB9NT, [G]

D4AHQ, Nove Herminovy, Slezsko [G]

Hans Woletz (killed in WW2), Nove Sady u Olmuouce, Olmütz

Fritz Woletz (1910-1989), Nove Sady u Olmuouce, Olmütz Liegnitz, Berlin, DE6827, D4RMQ, DL7AD [E], [G]

$\operatorname{EK} 4 \mathrm{CM}(2)$

?? Klaipeda

Vytautas Vanagas, Kaunas Lithuania Riga, DE1322, YL2BV [E] in 1929, LY1AA in 1933, [G]

EK4CE, Kowno Lithuania [E]

E. Bartininkas, Lithuania, DE0556, RY1MB, LL1MB [G][LY2YR]

?? Lietuva

W. Arnold Trembinski, Warschau, DE1068, SP3AT?, [G]

EK4SC, Herman Franke [CB1932]

Alfredo della Bona (-1984), DE0453, EK4SR(2), FS4SAC, EZ4SAC [J:7] Gebrüder Della Bona Vegetables and Fruits Imports, Saarbrücken, founded 1923, Alfredo became CEO in 1930 [CB1932][G], heard overseas [CQ-MB 7/31]

Rudolf Bluel (-1984), Saarbrücken, DE2699T, EZ4SAB, D4QBT [CB1936], D3KUT [RL1944], 9S4AL [E],

DL8AL [CB1959][G]

EK4SR(1), Alfred Woerner, heard overseas [CQ-MB 8/31]

EK4SZ, Fr. Meyer-Buchardt [CB1932][G]

EK4SM, Dittmar [CB1932][G], signals heard overseas [CQ-MB 3/31]

EK4SK, Georg Klos, Saarbrücken

signals heard overseas [CQ-MB 6/31] ??

Saar Radio Club e.V., Saarbrücken [CB1932]

Peter Kleemann, Saarbrücken, EZ4SUP [U], spelled Keemann in [CB1932, 1934], heard overseas [CQ-MB 8/31]

heard overseas [CQ-MB 11/31] ??

[CQ-MB 4/32] ??

Pavle Spinner, Zemun Belgrade, DE1215 [G]

EJ7DD, YU7UU

Josip Grbec, Ljubliana, DE1604, UN7SJ [G]

Mantovic, Sambor, DE1245 [G]

N. L. Novkovich, Zagreb, DASD member, chief operator of station UNP1 in Zagreb [E]

UN7GL

Charles Vohalsky, Vinkovsci, DASD member, [E]

EAAA

Johann Sax, DE1105S, OE3JS, [G]

Karl Götz, Vöslau Baden/Wien, DE1051S, OE3JT, D3JKS, [G]

EAKY

UO1JZ

EAKL

Karl Bausch, Bregenz München, DE1601W, DA2BS, DA2FL, DL1AY [E], [G]

UO1SL

UO1WG

Hans Furlan, DE1265W, UO8HF or Eduard Jaeger, DE1266W, UO7EJ (error in) [G]

Leonhard Frohn, St. Pölten Wiener Neustadt, DE0007P, UO7NA, Op. at EK4UAF, DL3PK, [G]

Robert Adler, Wien, DE1081S, [G]

EACM

Fritz/Richard Haas (-1981), Salzburg Wien, DE1103/DE6507, EAJI, OE1FH, OE1HRW [E], [G]

Julius Zwerina, Wien, DE1077W, OE1JZ, [G]

L. Schwendt, Wien Schwerin, DE1090S, [G]

Hans Wieder, Salzburg, DE1020S, DE1180W, UOWG, OE2HW, [G]

Franz Lehner, Linz/Donau, DE6475, OE081, OE3FL, [G]

Umlauff-Frankwell, Wien Leobersdorf, DE1092S, OE3HU, [G]

EAKL 


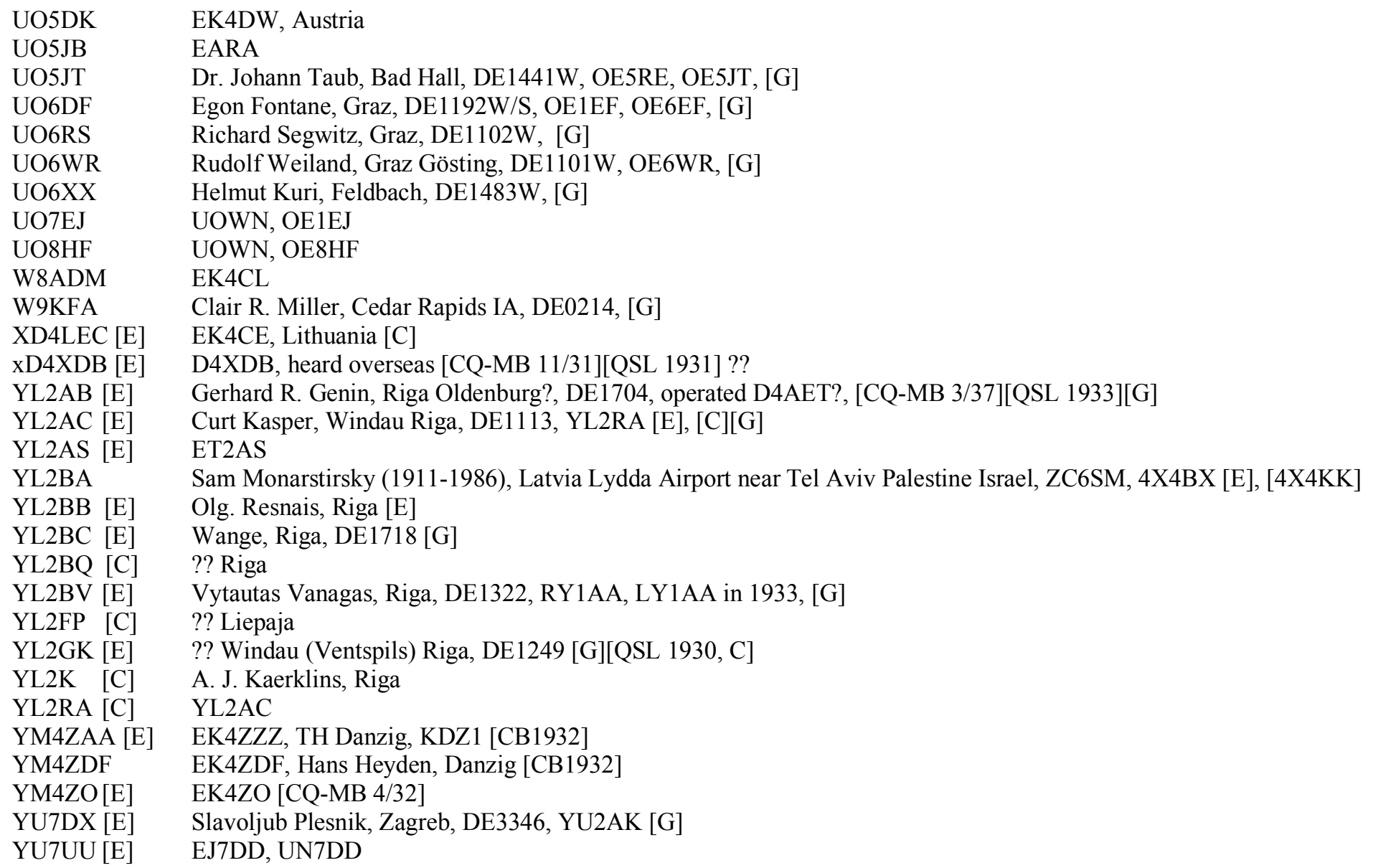


(3) 1924-1927:

\section{3a) Pre-1927 official "lis" calls of clubs, companies and educational institutions:}

$\mathrm{Ka} 0 \quad$ EK4ACU Dr. Erich Huth G.m.b.H. Berlin, inventor of the Hirsch direct reading wave meter [M 1927]

$\begin{array}{ll}\text { Ka1 } & \text { EK4ACY } \\ \text { Ka2 } & \text { EK4ACM } \\ \text { Ka3 } & \text { EK4ACL } \\ \text { Ka6 } & \text { EK4ACT } \\ \text { Ka7 } & \text { EK4ACS } \\ \text { Ka8 } & \text { EK4ACC } \\ \text { Ka9 } & \end{array}$
[Simon \& Goldsmith, 1913]

$$
\text { Physikalisch Technische Reichsanstalt Berlin [M 1927] }
$$

Telefunken Ges. f. drahtl. Telegr. m.b.H. Berlin [M 1927]

Telefunken Ges. f. drahtl. Telegr. m.b.H. Berlin [M 1927]

C. Lorenz AG, Berlin-Tempelhof [M 1927]

C. Lorenz AG, Berlin-Tempelhof [M 1927]

C. Lorenz AG, Berlin-Tempelhof [M 1927]

Dr. Joseph Benedict Engl (1893-1942), Berlin, emigrated to New York, USA in 1939. Jo Engl was in 1918 co-inventor of the talking film with Hans Vogt and Joseph Massolle at Tri-ergon

[J:1924 Versuchsender][Solomon, 2011]

$\begin{array}{ll}\text { Kb0 } & \\ \text { Kb1 } & \text { EK4AER } \\ \text { Kb2 } & \text { EK4ADD } \\ & \\ \text { Kb3 } & \text { EK4ADG } \\ \text { Kb4 } & \\ \text { Kb5 } & \text { EK4ACW } \\ & \\ \text { Kb6 } & \text { EK4ADH } \\ \text { Kb7 [E] } & \text { EK4ADE, Kc8? } \\ \text { Kb9 } & \text { EK4ADF } \\ \text { Kc0(1) } & \text { EK4ACP } \\ \text { Kc0(2) } & \text { EK4ACQ } \\ \text { Kc1 } & \text { EK4ACE } \\ \text { Kc2 } & \text { EK4ACZ } \\ & \end{array}$

Funktechnischer Verein, Funkfreunde Potsdam

Deutscher Radioclub (DRC) Fürstenwalde, EK4AER [J:1924 Versuchsender]

Denes v. Milhaly (1894-1953), Berlin, pioneer in television technology, invented in 1922 the Projectophon, a device for transmitting still images [J:1924 Versuchsender][M 1927][W]

"Exploration" Bodenuntersuchungs- und Verwertungs G.m.b.H., Berlin [M 1927]

Dr. Max Hausdorff, Berlin, Author [Hausdorff, 1925][J:1924 Versuchsender]

Deutsche Telefonwerke und Kabelindustrie A.-G., KB5, D2BP [RL-D2 1935], [M 1927]

[heard Wireless World 12/1925]

Funktechnischer Verein (FTV) Berlin-Lichtenberg, Kohnert [J:1924 Versuchsender]

Kb7 [E] EK4ADE, Kc8?, Funktechnischer Verein Berlin Charlottenburg W 9 [J:1924 Versuchsender][E]

Funktechnischer Verein, Gruppe Osram, Berlin [M 1927]

Telefunken Ges. f. drahtl. Telegr. m.b.H. Berlin, Fürstenbrunn, Wernerwerk, Prüfschuppen [M 1927]

Telefunken Ges. f. drahtl. Telegr. m.b.H. Berlin, Fürstenbrunn, Wernerwerk, Telefunken-Prüfwiese [M 1927]

Deutscher Radio-Klub e. V., D. R. C., Ortsgruppe Ringenwalde, Kreis Templin [M 1927]

Labor Dr. Siegmund Loewe (1885-1962), Berlin, studied with Max Wien in Jena, his company was expropriated In 1938 and Loewe emigrated to the United States. The company was returned to Loewe in 1949

[J:1924 Versuchsender][W]

$\begin{array}{ll}\text { Kc3 } & \text { EK4ACD } \\ \text { Kc4 [D] } & \text { EK4ACF } \\ \text { Kc5 } & \\ \text { Kc6 } & \text { EK4ACX } \\ \text { Kc7 [D] } & \text { EK4ADI } \\ \text { Kc8 } & \text { EK4ADC } \\ \text { Kdz1 } & \text { EK4ZZZ } \\ \text { Kd2 } & \text { EK4ADJ } \\ \text { Kd3 } & \text { EK4ADE } \\ \text { Kd6 } & \text { EK4AFA }\end{array}$

C. Lorenz AG, Neuendorf bei Zossen [J:1924 Versuchsender]

Funkgesellschaft (D.R.C.), Eberswalde [J:1924 Versuchsender]

Dr. Ing. Walter Dornig (1881-), Spandau [J:1924 Versuchsender]

Physikalisches Institut der Technischen Hochschule Berlin [M 1927]

Ortsgruppe Schöneberg, e. V. D. R. C. Op: EK4CI(1), Fritz Kunze [G]

FTV Experimental Station of the Berlin-Schlachtensee DASD Section, Kb7, previous calls Kxox, XOX [D]

YM4ZAA, Technische Hochschule Danzig

Ferdinand Schuchhardt, Berliner Fernsprech- und Telegraphenwerke A.-G. Berlin SO 16, [M 1927]

Hellmuth Schulz, F.T.V., Berlin Charlottenburg [J:1924 Versuchsender]

Deutscher Radio Club e. V. Berlin-Charlottenburg [M 1927]

Berlin-Wannsee, Ortsgruppe Wannseebahn?, Charlottenburg?

\section{Kd7 \\ $\operatorname{Kd} 9(1)$ \\ Kd9(2) EK4ADB

$\begin{array}{ll}\text { Kf8 } & \text { EK4ABB } \\ \text { Kg0 } & \text { EK4AAC } \\ \text { Kg1 } & \text { EK4AAA } \\ \text { Kg3 } & \text { EK4AAB } \\ \text { Kg6 } & \\ \text { Kg7 } & \text { EK4AAD } \\ \text { Ki0 } & \text { EK4ADW } \\ \text { Ki1 } & \text { EK4ABW } \\ \text { Ki2 [D] } & \text { EK4ABF }\end{array}$

Ki3 EK4AEL

Ki4 [E] EK4ADU

$\mathrm{Ki5}(1)$ EK4ACO

Ki5(2) EK4AES

Ki6 [D] EK4AEO

$\operatorname{Ki} 8(1)$

$\operatorname{Ki} 8(2)[E]$

Ki9(1) EK4AAT
Deutscher Radio Club e. V. Königs Wusterhausen [J:1924 Versuchsender]

Deutscher Radio Club e. V. Wannseebahn H. Kraus [J:1924 Versuchsender]

Funkverein e. V., Gruppe Elektrowerk A.-G., Berlin W62 [M 1927]

Funktechnischer Verein, Gruppe Elektrowerke, Berlin W 62

Physikalisches Institut der Universität Greifswald [M 1927]

Telegr.-Techn. Reichsamt, Abt. Versuche Berlin [M 1927]

Telegr.-Techn. Reichsamt, Abt. Funkwesen Berlin [M 1927]

Telegr.-Techn. Reichsamt, Abt. Funkwesen Berlin [M 1927]

[heard Wireless World 12/1925] ??

Telegr.-Techn. Reichsamt, Abt. Funkwesen Döbnitz [M 1927]

Funktechnischer Verein e. V., Ortsgruppe Wernigerode [M 1927]

Physikalisches Institut der Techn. Hochschule Hannover [M 1927]

Bad. Gesellschaft für Radiotechnik, Werner Manecke Durlach, Karlsruhe [J:1924 Versuchsender]

Oberdeutscher Funkverband (Badische Gesellschaft für Radiotechnik), Karlsruhe, Op: Wilhelm Doering, EK4OM

Deutsche Betriebsges. f. drahtlose Telegr. m.b.H., Hamburg [M 1927]

Verein der Funkfreunde Schlesiens, Liegnitz [J:1924 Versuchsender]

Telefunken Ges. f. drahtl. Telegr. m.b.H. Berlin, Leipzig [M 1927]

Physikalisches Institut der Universität Leipzig [M 1927]

FTV, Dr. Richard Wohlstadt, Hamburg [J:1924 Versuchsender][D]

Verein der Funkhändler, Darmstadt - in 1925 [J:1924 Versuchsender]

Südwestdeutscher Radioclub (Verein der Funkfreunde Darmstadt, Darmstadt-Starkenburg), Op: S. J. Tyrrell, [C]

[heard Wireless World 12/1925]

Institut für Schwachstromtechnik der Universität Dresden [M 1927] 
Ki9(2) EK4ABX

Kj1 [D] EK4ABE

$\mathrm{Kj} 2$ EK4AAO

Kj3 EK4ABS

(Kj4 EK4ABV

$\mathrm{Kj} 5$ [E]

$\mathrm{Kj} 5(2)[\mathrm{D}]$

Kj6

Kj7 EK4AAJ

Kj8 EK4AAN

$\mathrm{Kj} 9$

Kk0 [E] EK4AAK

Kk1 EK4ABO

Kk2 EK4ABV

$\mathrm{Kk} 3(1)$

$\mathrm{Kk} 3(2)$ EK4AAW

Kk4(1) EK4AET

Kk4(2)[E] EK4ABR

Kk5 [D] EK4ABP

Kk6 EK4ABL

Kk7(1) EK4ABG

$\mathrm{Kk} 7(2)[\mathrm{D}] \mathrm{EK} 4 \mathrm{ABR}$

Kk8 EK4ABT

Kk9 EK4AAV

K10 [D] EK4AAP

K12 EK4AEM

K13 EK4ABM

K14 [D] EK4ABI

K19 EK4ABU

Km3 EK4ADY

Km6 EK4AEN

$\mathrm{Km} 9$

$\mathrm{Kml}$

Kn0 EK4AEK

Kn1 EK4AEA

$\mathrm{Kn} 2$ EK4AEB

$\mathrm{Kn} 3$ EK4AEC

Kn4 EK4AED

Kn5 EK4AEF

Kn6 EK4AEG

$\mathrm{Kn} 7$ EK4AEH

Kn8 EK4AEI

Kn9 EK4AEJ

Ko1 EK4ACA

Ko2 EK4ABH

Kpl [E] EK4DBA

Kp0 EK4ADZ

Kp1 EK4ABJ

Kp3

Kp4 EK4AAZ

Kp5(1) EK4ACG

$\mathrm{Kp} 5(2)$ EK4ACJ

Kp6(1) EK4ACI

Kp6(2)[D] EK4ACI

$\begin{array}{ll}\text { Kp9 } & \text { EK4AAY } \\ \text { Kq1 } & \text { EK4ABK } \\ \text { Kq2 } & \text { EK4AEY } \\ \text { Kq3 } & \\ \text { Kq4 } & \\ \text { Kq5 [E] EK4ABN } \\ \text { Kq7 [E] EK4AEQ }\end{array}$

Gesellschaft der Funkfreunde Hannover [M 1927]

Westdeutscher Funkverband e. V., Münster, Op: Willi Stecher, DE0037 [G]

Funkwissenschaftliche Vereinigung Netzschkau, I.A.R.U.

Rhein. Technikum Bingen (Rhein) Lorch [M 1927]

Verband der Funkfreunde Schlesiens ??) see Kk2

Owin Radio Apparate Fabrik, Hannover Talstr. 6, QSL [C] Op: Ernst Plathner (1899-1971), DE0021K [G]

Ernst Plathner (1899-1971), founded Owin, Hannover, DE0021K [D]

??, Op: EK4CI(1), Fritz Kunze [G]

Kyffhäuser-Technikum Frankenhausen [M 1927]

Staatliche Gewerbeakademie, Schillerstr. 7, Chemniz [TUC][M 1927]

Gesellschaft der Funkfreunde, Hannover, Op: Ernst Plathner, DE0021K [D]

Radio Vereinigung Gera, Thüringen, Op: Kurt Müller Liebschwitz (Elster) Gartenstr. 1

Gesellsch. v. Freunden der Radiotelephonie und Telegraphie in Marburg (Lahn), Otto Rottmann [D]

[M 1927]

Verband der Funkfreunde Schlesiens e. V., Breslau [M 1927]

Carl Mecke, Giessen [J:1924 Versuchsender]

Physikalisches Institut der Universität Giessen [M 1927]

Technikum Mittweida [M 1927]

Südwestdeutscher Radio Club, Frankfurt a. M., Op: F. Bödigheimer, [C][heard Wireless World 12/1925]

Südwestdeutscher Radioclub, Frankfurt [heard Wireless World 12/1925][J:1924 Versuchsender]

Physikalisches Institut der Universität Marburg [J:1924 Versuchsender]

Anton Otto Klotz [M 1927]

Ferdinand Boedigheimer, EK4AB(1) Frankfurt [D][heard Wireless World 12/1925]

Physikalisches Institut der Schles. Fried.-Wilhelm Universität Breslau [M 1927]

Polytechnikum Friedberg, Hessen [E][M 1927]

Funktechnische Vereinigung Plauen-Vogtland [D][J:1924 Versuchsender]

Phys. Staatslabor der Universität Hamburg [M 1927]

Ingenieur Ferdinand Schneider G.m.b.H (1866-1955), Fulda [M 1927][W]

[heard Wireless World 12/1925]

Funkvereinigung Halle [D]

Technische Hochschule Breslau

Stassfurter Licht- und Kraftwerke A.-G. Stassfurt [M 1927]

Radioröhrenfabrik G.m.b.H., Vulkan, Hamburg [M 1927]

[heard Wireless World 12/1925] ??

[CQ-MB 7/27] ??

Junkers-Flugzeugwerke A.-G. Dessau, Flugfunkstelle [M 1927]

Junkers-Flugzeugwerke A.-G. Dessau [M 1927]

Junkers-Flugzeugwerke A.-G. Dessau, Flugfunkstelle [M 1927]

Junkers-Flugzeugwerke A.-G. Dessau, Flugfunkstelle [M 1927]

Junkers-Flugzeugwerke A.-G. Dessau, Flugfunkstelle [M 1927]

Junkers-Flugzeugwerke A.-G. Dessau, Flugfunkstelle [M 1927]

Junkers-Flugzeugwerke A.-G. Dessau, Flugfunkstelle [M 1927]

Junkers-Flugzeugwerke A.-G. Dessau, Flugfunkstelle [M 1927]

Junkers-Flugzeugwerke A.-G. Dessau, Flugfunkstelle [M 1927]

Junkers-Flugzeugwerke A.-G. Dessau, Flugfunkstelle [M 1927]

Ostdeutsche Funkfreunde, Königsberg, Hugo Fagien KpL [D]

Badische höhere technische Lehranstalt Karlsruhe [M 1927]

Hugo Fagien (-1953) [heard Wireless World 12/1925]

Funktechnischer Verein FTV, Bezirksgruppe Magdeburg [J:1924 Versuchsender]

Technikum Strelitz [M 1927]

Radiovereinigung Leipzig [D]

Verein zur Förderung des Funkwesens e.V., Funkverband Niederdeutschland e.V., Ortsgruppe Osnabrück [D][J:1924 Versuchsender]

Institut der Radiokunde, Bremen [J:1924 Versuchsender][M 1927]

Radiotechnischer Verein "Bergisch Land" Barmen [E][M 1927]

Uni Bonn?, Betriebsleiter: Dr. Wilhelm Ludolf Schmitz, EK4ACI (1899-1973)

Verein der Funkfreunde, Mülheim - Ruhr - Broich, Dr. Wilhelm Ludolf Schmitz, EK4ACI (1899-1973)

[J:1924 Versuchsender]

Ingineur-Akademie Städt. Polytechnikum [M 1927]

Elektrotechnisches Institut der Ingenieur-Akademie Wismar

Deutscher Radioclub D.R.C., Ortsgruppe Braunschweig, Städt. Berufsschule

Mitteldeuscher Radioclub, Annaberg Erzgebirge

Funkvereinigung e. V., Erfurt

Südwestdeutscher Radioclub, Cassel, Op: Franz Noether, EK4KL [J:1924 Versuchsender]

Deutscher Radioclub, D.R.C., Cottbus “K97”, [C][Der Radioamateur, 34, 8/1925, p829-831] 


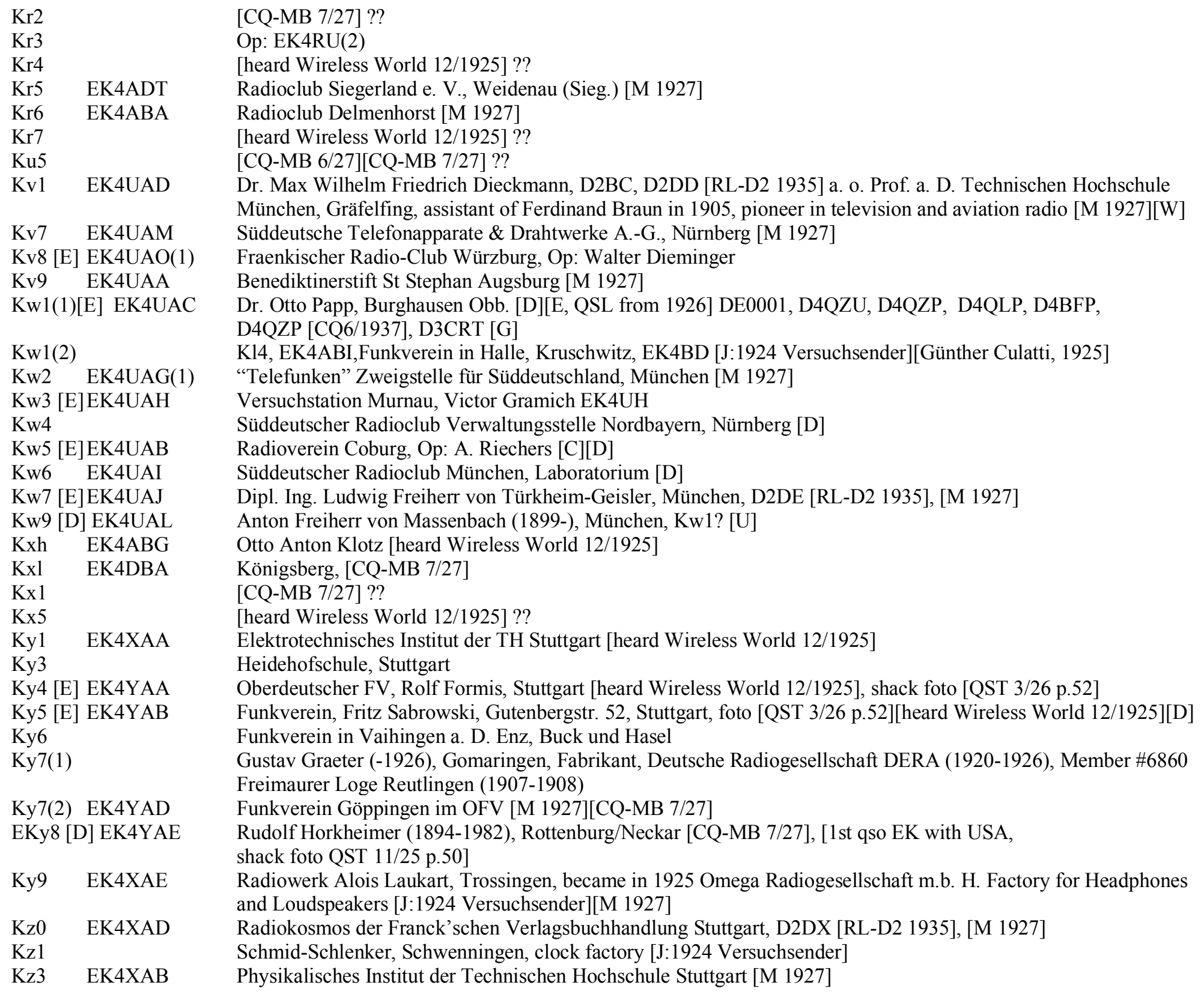




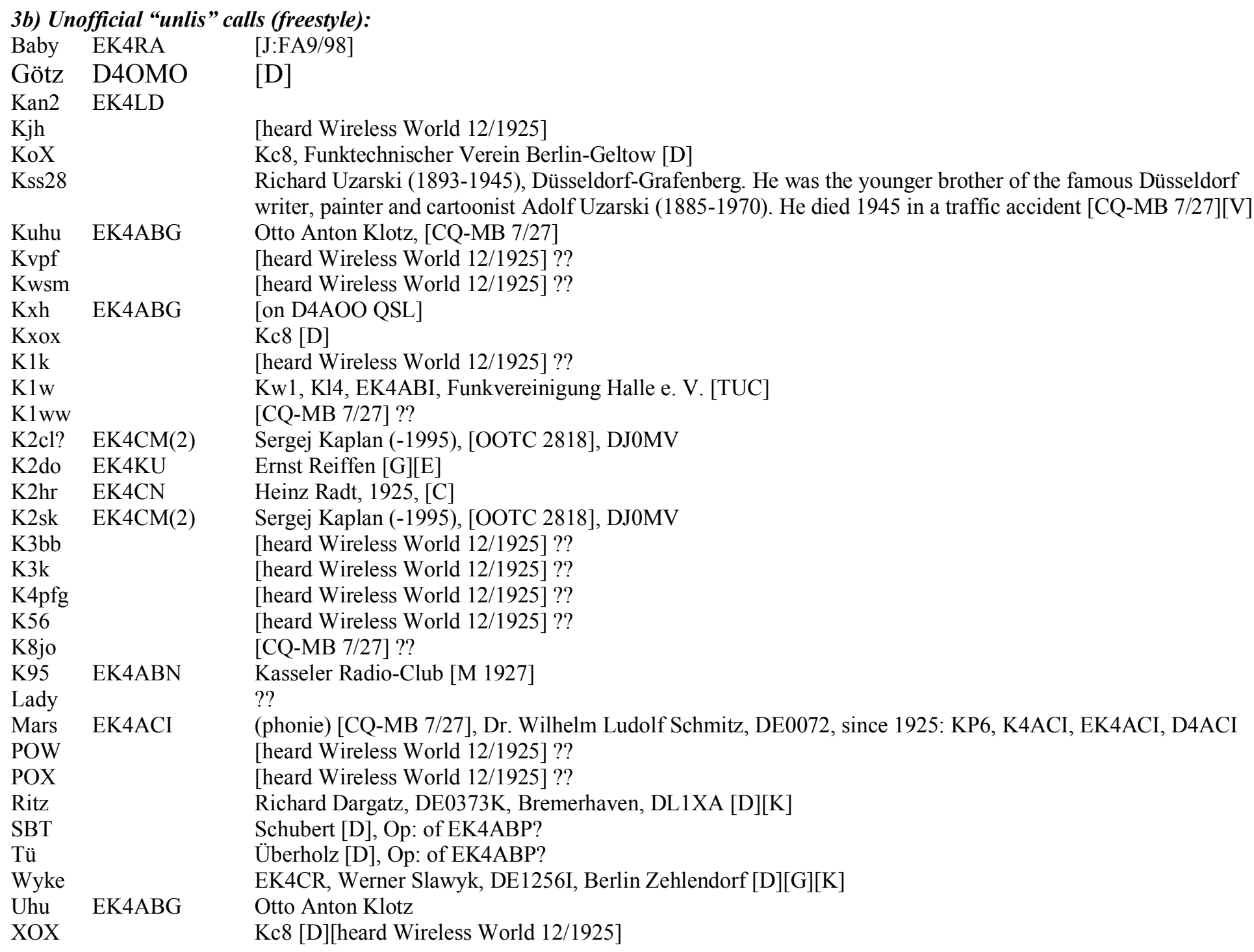


(4) After 1949 - Calls of pre-1933 stations and holders of DE numbers

For the pre-1933 stations, the second column lists in bold the call under which information can be found in the previous sections.

DC7CF

DC7UV

DF1MD [E]

DF2MF [E]

DF2MG [CB1981] DL7BO

DF2TU DL6SC

DF3BX

DF3FS

DF3SR

DF3XX

DF4XN

DF7IL

DF7RD

DF8JL

DF8KS

DF8TG

DF9LW

DJ0MV

DJ1AK [E]

DJ1DA [E]

DJ1EG

DJ1FT

DJ1HE

DJ1HM

DJ1HT(1)

DJ1HT(2) [E]

DJ1HX

DJ1HY

DJ1IL [E]

DJIIM [E]

DJ1IX

DJ1KW

DJ1LD

DJ1LW

DJ1MC

DJ1MP

DJ1NA

DJ1QQ [E]

DJITU

DJ1VE [CB1957]

DJ1WT [E]

DJIZG

DJ2AZ

DJ2BC [E]

DJ2FZ

DJ2GQ [E]

DJ2HL

DJ2IB [E]

DJ2IL

DJ2IW [E]

DJ2OY

DJ2PM

DJ2QZ [E]

DJ2RG [E]

DJ2SR [E]

DJ2UU [E]

DJ2WX

DJ3BV

DJ3CO [E]

DJ3FE [E]

DJ3HW [E]

DJ3IX

DJ3IZ [E]
EK4AP

EK4IP

DE6316

DE3114

D4LWL

DE6503

DE1375D

EK4KA

DE6824

EK4TV(1)

DE2099G

DL6WJ [E] DE6834

DE6722

DE1887H

DE6147

DE2649F

DL1GW DE2861V

EK4CM, PAOC

D4IFF(1) [E]

DE6707

DE3571

DE1512I

DE3654

DE3990

EK4LAA

DE12774

OE8FG DE6672

DE6917

D4BYH

EK4UI

DE3292

DE6755

DE3899I

DE3936

DE3210

DE2528T

DE6340

D4CWA

DE3617

DE3488

DE12012

DE1955H

D4BOG

DE2078D

EK4TM

DE14442

DE6866

DE3512

DE6313

EK4HX

EK4TJ

EK4BC

EK4KK

DE2679T

DE2411

DE7353

DE3753

DE3576

DE2572K

DE3477A

DE2847

DE3818

DE3189
(1904- 1985) [E:photo]

$(-1985)$

Wolfgang Schmidt, Mannheim Augsburg [G]

Hubert Schlösser, Blaichach Stuttgart [G]

$$
(-1985)
$$

Heinrich (Heiner) Treiber (-2016), Ravensburg Stuttgart [G]

Walter Noack, Magdeburg Bad Iburg, D4JTD, D4LNQ, D4BBD [E], D4FHD [E], [G] $(-1980)$

Artur Kirn, Sersheim [G]

$$
(-\sim 1990)
$$

Heinrich Nerlich, Breslau [G]

Rudi Christmann, Pfortzheim Darmstadt [G]

Karl Pommer, Eitdorf Darmstadt [G]

Boris Hausmann, Wuppertal Erkrath [G]

Gunther Benkert, Berlin St. Augustin [G]

Wolfgang Häusler (-1986), Berlin Breslau Balingen, left DASD 1945 [G]

Hermann Selmer (-1985), Flensburg [G]

(1938) (-1995)

Wilhelm Granrath, Eschweiler [G]

Johann Mayer, Augsburg Neusäß [G]

Emil Diederich, Köln, D4BQI, D4YQI, D4APR [G]

Toni Grosskämper, Köln [G]

"Peter" Edgar Krause, Berlin Hamburg, D4XCF, D5TM, D1AB, DA7RA [E], [G] (-1955)

Hermann Dumke, Essen-Schonnebeck [E]

Ing. Felix Gaggi, Klagenfurt [G]

Dr. Joachim Wischmann, Eberswalde Lahr Kassel [G] $(-1986)$

$$
(-1972)
$$

Fritz Huth, Lindenholzhausen [G]

Helmut Liebrecht, Hannover [G]

Josef Ollfisch, Aachen [G]

Karl Heinz Koch, Recklinghausen Düsseldorf [G]

Walter Ernst, München [G]

Ernst Müller, Frankfurt/Rödelheim Stockstadt [G]

Johann Ehrlinger, Nürnberg [G] $(-1985)$

Heinz W. Boddenberg, Karmen Unna Mettmann [G]

Otto Dummer, Oldenburg Kohlscheid Alsfeld Aachen [G]

Fritz Zöller, Hamburg Wichernsgarten [E]

Herbert Lennartz, Essen Tübingen [G]

$$
(->1994)
$$

Dr. Günther Lange-Hesse, Göttingen Lindau/H., D4UVD [E], D4VLV? [G] (- 1985)

Viktor Homberg, Schwäbisch Hall, Cleebronn [E]

Friedrich Georg Bennertz, Solingen [G]

Andreas Nagel, Himmeltal Karlsruhe Spielberg [G]

Andreas Drewing, Essen [G]

$$
\begin{aligned}
& (-1983) \\
& (-1986) \\
& (->1994) \\
& (-1988)
\end{aligned}
$$

Albert Ungeheuer, Hofheim/Ts. [G]

Ernst Münter, Nürnberg [G]

Hansgeorg Bähr, Hanau/Main [E]

Kurt W. Dittmann (-1980), Berlin Bonn,

Karl Wismath (-1988), Regensburg [G]

"Max", "Fred" Alfred Jung (-1962), Freiburg/Sa. Karlsruhe, DK8HQ [G][J:14]

Heinrich Papenburg (-1987), Königsberg Edemissen [G]

Bernhard Kaiser, Hörstel Rheine Köln [G]

Georg Stiegler, Regensburg [G]

Otto Wagner, Breitenhausen Regensburg München Niederwinkling [G] 
DJ3LE [E]

DJ3LL [E]

DJ3MG [E]

DJ3NF [E]

DJ3RM

DJ3RX

DJ3UM

DJ3UN

DJ3VN [E]

DJ3VR

DJ3WC [E]

DJ3WP [E]

DJ3ZY?

DJ4AV [E]

DJ4DN [E]

DJ4HZ

DJ4LC [E]

DJ4PH

DJ4QA

DJ4QI

DJ4QU

DJ4SA

DJ4UH

DJ4UN

DJ4WN [E] HB9AWV

DJ4XP

DJ5II

DJ5LK

DJ5OA

DJ6CK [E]

DJ6DG

DJ6LQ

DJ6QG

DJ6RH

DJ6UC

DJ7AV

DJ7OX

DJ7SK

DJ7UB

DJ8CR [E]

DJ8HF [E]

DJ8NZ

DJ8SG [E]

DJ9CJ [E]

DJ9EN

DJ9GD [E]

DJ9YJ

DK2FR

DK2OY

DK3HP

DK3MN

DK3QU

DK3UO [E]

DK3ZR

DK4AU

DK4BA

DK4OG [RL77] DL3MG

DK4QZ

DK4UA

DK5BX

DK5DB

DK5EL [E]

DK5GA [E]

DK5HX

DB3ZU?
EK4EN
D4FQF
(- 1992)
$(-\sim 1979)$

DE3523

DE3566

DE1772U/I

DE6687

EK4GI

D4EWW

DE6997

D4ZQW

DE3106

EK4ES

DE0085Y

DE2674R

D4LLL(2)

DE2487U

D4XIT

EK4AR(1)

DE1820A

DE6648

DE2704C

DE6098

EK4FI

CT2AB [CB1932]

EK4AS DF4UN?

Heinz Ahlburg, Braunschweig Bad Pyrmont [G]

Fritz Franz Kreh, Mariendorf Varel, D4AYA [G]

Walter Krieger, Leipzig Köln [G]

Benno Bauer, Regensburg [G]

$(-1963)$

$(->1994)$

Erwin Greiner (1921-2013), Rommelshausen Kernen/Remstal [E] $(-\sim 1985)$

Reinhold Ungefroren (-1982), Bielefeld Eschweiler Steinhagen, started 1934 $(-1986)$

W. Schade, Danzig, Op: EK4ZZZ [G]

Alois Diry, Amberg Alzenau Regensburg, D3GDR [G] $(-1974)$

Herbert Zimmermann, Weimar Köln, D4BWL(2)?, [G] $(-1982)$ $(->1995)$

Walter Kannigowski, Heiligbeil Bochum, D4CAA, D4ARE, Op: D4ACA [G]

Heinz Hirscher, Amtzell [G]

Willi Mohwinkel (-1967), Potsdam Villingen [G]

Johannes Baumgart, Kleinkrotzenburg Tilsit [G]

$(-1959)$

(1893-1968)

DE6571

DE2823H

DE2617N

DE6158

DE3908

EK4CH

DE2963U

DE3442

DE1914H

D4JGV

EK4GV

EK4PO

DE2968F

DE8161

DE14434

DE3394

DE6565

DE3384

DE1869T [TUC] Dipl. Ing. Eberhard Meyer, Ludwigshafen Frankfurt/Main München Hamburg, D4BYT

DE2267N Horst Tränkle, Stuttgart, D3DEN [G]

DE6317

DE2871

EK4AZ

DE6459

DE3573

DE2597C

$\mathrm{DE} 0075 \mathrm{~F} / \mathrm{U}$

DE2816H

DE1864H

EK4AU

DE6412

DE6386

DE6627

DE2443T

DE5555H

DE2795Y

DL3ND [E]

?

EK4YAE, Ky8

DE6622
[G]

Eugen Breuninger, Ulm München Heidenheim [G]

Ulrich Voß, Düsseldorf Bonn Essen [G]

Anton Schleich, Ay Neu Ulm Land [G]

Karl Heinz Köpf (-1971), Ulm Manheim [G]

Eberhard Graf von Hardenberg (-1983), Überlingen [G] $(-\sim 1963)$

Rudolf Mahr (-1980), Leipzig Winnenden [G]

Franz-Joseph Lauer, Kaiserslautern [G]

Emil Lienseid, Essen, D3GRH [G]

$$
\begin{aligned}
& (->1995) \\
& (-1983) \\
& (-1976) \\
& (-1991)
\end{aligned}
$$

Otto Schuhmacher, Idar-Oberstein [E]

Willi Nietmann, Bueren [E]

Friedrich Ebel, Brandenburg/Havel Aurich [G][E]

Peter Pius Seitz, Gnotzheim [G][E]

Gerhard Söhn, Düsseldorf, POW in Russia until 1961 [G]

Walter Misch, Mannheim Hockenheim [G]

Felix Prost, Horrem Essen [G]

$$
\text { (- 1990) }
$$

Manfred Petersen, Schleswig [G]

Erwin Weiler, Stuttgart Göppingen [G]

Erwin Hübner, Jüterbog München, D4BUL, D4HAC [G]

Ernst Friedrich, Berlin Fröndenberg [G]

Herbert Feldhaus, Wuppertal Hamburg [G]

Werner Wille, Halle Köln Harburg Hanau Italien, D3DIH, DA3GB [G] (1912-1972)

Johann Pasch (-1988), München Köln Budaörs, HAF31 [G]

Kurt Gubbe, Stettin Hannover Bevensen [G]

Wolfgang Zimmermann, Dresden Mosbach [G][E]

Dr. H. Keßler, Gießen [G]

Wolfgang Krüger, member FWGM [G]

Erwin Bönisch (-1983), Danzig/Langfuhr Brilon, YM4AL

Otto Schopen, Düsseldorf-Oberkassel Krefeld [E] (1894-1982)

Fritz Frank, Ravensburg Lüneburg [G][E] 


\begin{tabular}{|c|c|c|}
\hline DK5LI [CB92] & DL1GJ [CB50] & DE2454 \\
\hline DK6BO & & DE6817 \\
\hline DK6CS & & DE1825P \\
\hline DK8QN & & DE2486 [TUC \\
\hline \multicolumn{2}{|c|}{ DK9FA DM2FSO, DL8QA } & DE1894C \\
\hline DK9LG & DL3UT $(-1956)$ & ? \\
\hline DK9NQ [E] & DL6DU [E] & ? \\
\hline DL1AA [E] & & DE2783B \\
\hline DL1AC(1) [CB5 & $1-55]$ & DE6558 \\
\hline DL1AC(2) & & $\mathrm{DE} 1980 \mathrm{~F} / \mathrm{T}$ \\
\hline DL1AD [E] [CB & $51-53]$ & DE2371T \\
\hline DL1AJ $[\mathrm{E}]$ & & DE8202 \\
\hline DL1AM [E] & & DE6899 \\
\hline DL1AO[CB1952 & DL1AZ, I1UNO & EK4GC \\
\hline DL1AP [E] & & D4CLT \\
\hline DL1AR [E] & & DE6837 \\
\hline DL1AT & & DE3770 \\
\hline DL1AV(1) & DL2WE [E] & EK4GW \\
\hline $\operatorname{DL} 1 \mathrm{AV}(2)[\mathrm{E}]$ & DL1BF 1949 & DE6428 [E] \\
\hline DL1AW & & $\mathrm{DE} 2610 \mathrm{P}[\mathrm{E}]$ \\
\hline DL1AX & & DA2DH $[\mathrm{K}]$ \\
\hline
\end{tabular}

\begin{tabular}{|c|c|}
\hline DL1AY [E] & DE1601W \\
\hline DL1AZ(1) [E] [CB1949-58] & DE2228P \\
\hline \multicolumn{2}{|c|}{ DL1AZ(2) $>1958$, DL1AO, I1UNO EK4GC } \\
\hline DL1BA [E] & DE3204 \\
\hline DL1BB & DE6547 \\
\hline DL1BC [E][CB1951] & DE6802 \\
\hline DL1BD $[E]$ & D4BLP \\
\hline DL1BE & DE6877 \\
\hline DL1BF [E] 1949 DL1AV [1956] & DE6428 [E] \\
\hline DL1BG & DE3473 \\
\hline DL1BH [E] & DE6513 \\
\hline DL1BM [CB1951] & DE2974O [E] \\
\hline DL1BO [E] & DA1BE $[\mathrm{E}]$ \\
\hline DL1BP & DE7244A [E] \\
\hline DL1BQ & DE3663O \\
\hline DL1BR [E] & EK4GK \\
\hline DL1BS [E] & DE8448 [E] \\
\hline DL1BT & DE5368J \\
\hline DL1BU $[E]$ & DE7016 \\
\hline DL1BV & DE7015 \\
\hline DL1BW(1) [E] & DE6752 \\
\hline DL1BW(2) & DE3736 \\
\hline DL1BY & D4HIK \\
\hline DL1BZ & DE6850 \\
\hline
\end{tabular}

$\begin{array}{ll}\text { DL1CA } & \text { DE6518 } \\ \text { DL1CB } & \text { D4FTL } \\ \text { DL1CC } & \text { DE0070L } \\ \text { DL1CD [E] } & \text { DE6006 [E] } \\ & \\ \text { DL1CE } & \text { DE6413 } \\ \text { DL1CF [E] } & \text { DA3PN } \\ \text { DL1CH } & \text { DE3877 }\end{array}$

Jens Roschrock, Bremen [G]

Ewald Kadlobski (-1989), München [G]

Herbert Hartmann, Weimar Bielefeld Neuenkirchen Göttingen Berlin, D3AVN,

D3FND, D3FAF [G]

see DL8QA

Gerhard Lippok, Mittelsdorf, [OOTC 2708] [E]

Walter Freiss (->1994), Siegburg Troisdorf Dormitz Lindlar RL1994, [E]

Hans Heinrich Bachmann, Hausen über Witzenhausen [G]

Ulrich Benkmann, Kassel [G]

Heinz Schifferdecker [G]

Edmund Blume (-2012), Kassel [G]

Ewald Heerd, Bad Nauheim [E]

Heiner Reckewell, Kassel Goslar, DA3PW [G][E]

$(-1985)$

$(-1980)$

Heinz Günther Ballauf (-<1962), Frankfurt/M Bad Nauheim Norderney, DK3AA,

DA3AR, DA3FM [G]

Hans Schütze, Görlitz Gräfelfing [G]

(1904-1984)

“Dick” Horst S. Dorn, Ismaning München, DA2FB [G]

Hans Kern (-1953), München, D4TSP [G]

"Jonny" Hans Haberl (1924-2016), Markt Grafing, [K]. Member of a catholic resistance group "Vierergruppe" against the Nazi regime, who built clandestine radios in Munich.

The group, led by Walter Klingenbeck, was caught in 1941, and in 1942 three of them were sentenced to death, and one, Erwin Eidel, to prison. Klingenbeck was executed in 1943, the sentences of Haberl and Daniel von Recklinghausen (1925-2011) --a US citizen, later a pioneer in stereo technology with H. H. Scott in the US-- were converted to prison. The group was liberated in 1945 from the Stadelheim prison by US troups.

Haberl run a radio repair shop in Markt Grafing until 1983 [Hans Haberl W].

Karl Bausch, München Bregenz, UOOSK, DA2BS, DA2FL [G]

Alois Nöbauer, München Hohenschäftlarn, D4ADX, D4BPP, D4MZP [G][E] $(-1985)$

Heinz Pankow (-1996), München, D4TNP, DA2BV, DA2VA [G]

"Toni" Gerhard Merz, Hamburg Heide, DA2PA [B]

Karl Kümmelschuh, München [G][E] (-1950)

Johann Lutzenberger, München, D3JZP, DA2ZP [G]

"Dick" Horst S. Dorn, Ismaning München, DA2FB [G]

Albert Lauber, Karlsruhe, D4FUX 1947, DA1BB [G]

Erich Burgard (Jupp), Karlsruhe, DA1BC [G]

Karl Biehler, Sunthausen-Schwarzwald Schollbronn [G]

Theodor Flick, Karlsruhe [E]

Arnim Henglein, Karlsruhe [E]

Alfred Auchter, Karlsruhe, DA2IB? [G]

D4WXD [E] (-1966)

Kuno Huber, Karlsdorf bei Bruchsal Woerth [E]

Graf von Schwerin, DE7358, D4AKA, D4AYV, member FWGM [G]

Günter Schwarzbeck, Heidelberg Berlin Schönau Frankfurt/M, DA1DA [K], [G]

Walter Brischke, Brieg Eltmannshausen Marburg, DA3RF [G]

Wilhelm Entzeroth, Frieda/Werra, D3GWA [G]

Eugen Wachholz, Bremerhaven Wesermünde, DA5XR, [G] $(-\sim 1967)$

Hans Vizethum (-1987), Wiesbaden, DA3HA, directed Eiserne Hand Abwehrstelle in Paris during WW2. He trained Alfred Meiler alias Walter Köhler (1891 Nijmegen 1966 New York), the famous triple agent [West, 2019]. Joined the BND after WW2 and retired in the 1960ies [Vizethum, 1985] [G][E]

Friedrich Pape (-1975), Offenbach, DA3TI [G] $(-1970)$

Dr. Theodor Stolte (-1983), Eisenach Mahlberg [G]

Dr. Karl Heinz Fendt, Berlin Zehlendorf Spandau Spangenberg Melsungen, D4CMF [G][E]

Wlater Gramatte, Kassel [G]

Harald Lindner, Immenhausen [E]

Hans Joachim Thiessen, Pocking [G] 


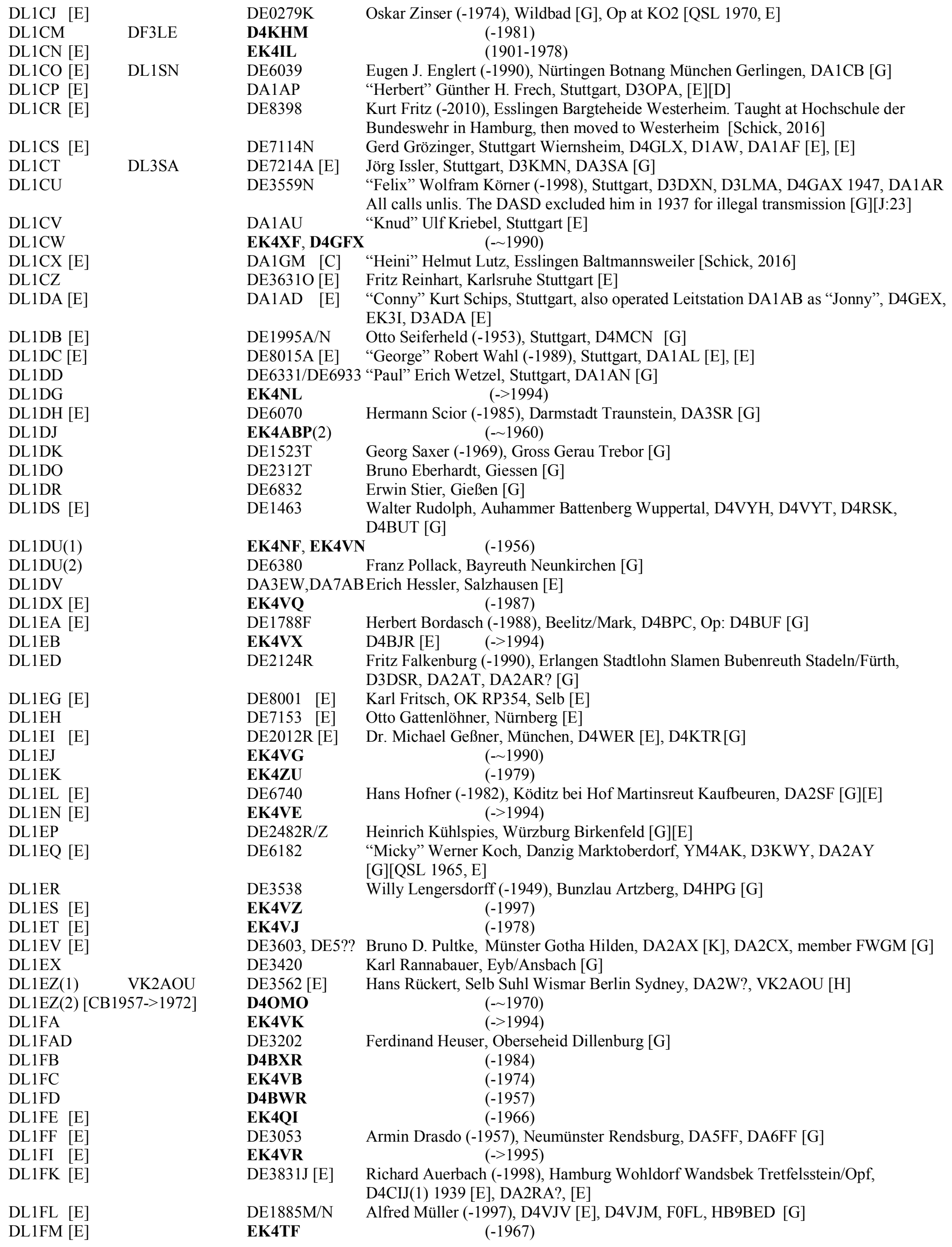




\begin{tabular}{|c|c|c|}
\hline DL1FN [E] & DE2723Y & Johannes Frank, Danzig Kiel, YM4AG, D4CZP [G] \\
\hline DL1FP [E] & DE3268 & Walter Jönck, Kiel Holtenau, D3HKV, DA6FQ [G][E] \\
\hline DL1FQ [E] & EK4TQ & $(-1983)$ \\
\hline DL1FS & $\mathrm{DE} 3685 \mathrm{~V}$ & Heinz Hornig, Rendsburg, not in [G] - info from LY1AD 1939 QSL in [C] \\
\hline DL1FT & DE3683 & Werner Schnepel, Rendsberg Nürnberg [G] \\
\hline DL1FU & DE3679 & Wilko Ukena, Rendsburg Rottau/Chiemgau [G] \\
\hline DL1FV [E] & DA7FI,DA6TX & "Bruno" Jürgen Sick, Hamdorf Berlin [E] \\
\hline DL1FW [E] & DE6266 & Heinrich Fehmann, Schleswig, DA6FC [G] \\
\hline DL1FX. [E] & DA6FJ & Willi Wallatis, Kropp bei Schleswig [E] \\
\hline DL1FY [E] & DE6713 & Oscar Gohn (-1987), Berlin Schleswig, DA6FT [G][E] \\
\hline DL1FZ [E] & DE3887 & "Pat" Dr. Ing. Fricko Freiherr von Rautenfeld (-1978), YM4AV [E], DA6FZ [E], [G] \\
\hline DL1GB [E] & DE3544 & Walter Finster, Mönkeberg [G] \\
\hline DL1GC [E] & DE3097 & Wilhelm Hill, Kiel, D4RIT [G,E] \\
\hline DL1GD [E] & EK4QA & $(-\sim 1975)$ \\
\hline DL1GF & DA6BD [E] & “John” Johannes Hassler, Kiel [E] \\
\hline DL1GH & DE1632G & Werner Heissig (-1992), Görlitz Bersenbrück Oldenburg Bremen, DA5BX [G] \\
\hline DL1GJ [CB50] DK5LI [CB92] & DE2454 & $\begin{array}{l}\text { Karl-Heinrich Cehak }(-<1995) \text {, Chemnitz Itzehoe Husum Rantrum Schülp Rendsburg } \\
\text { Bremen, D4VLU [CQ6/1937], [G][E] }\end{array}$ \\
\hline DL1GM[E] & DE6807 & Jürgen Bennöhr (Ben), Kiel Hassee Parching Aumuehle Fahrdorf [G][E] \\
\hline DL1GN [E] & EK4KUE & $(-1976)$ \\
\hline DL1GP [E] & DE2155V & Hans-Hermann Horn, Flensburg Mittweida, DA6XY [G] \\
\hline DL1GR [only CB1949/50] & DE3959 & Reimer Emeis ( -1950), Flensburg [G] \\
\hline DL1GS [E] & DE1753B [TUC] & Gerhard Senkel, Stolp Langenballig [G] \\
\hline DL1GT [E] & DE2157V & Karlheinz Bengs, Flensburg, D4VZV, DA6MG [G] \\
\hline DL1GU & DE6533 & Ludwig Thiel, Neustadt [G] \\
\hline DL1GV [E] & DA6MC & Hei Soujon, Flensburg, D4MAC [E] \\
\hline DF9LW & DE2861V & Hermann Selmer, see DF9LW \\
\hline DL1GX & DE1486K [E] & Gustav Blaeser, Northeim/Hannover Neustadt i. H., D4BGK [E] [CB1934], [E] \\
\hline DL1GY [E] & DE1963R & $\begin{array}{l}\text { Dr. Alexander Pracher, Aschaffenburg Koblenz Pelzerhagen Neustadt/Holstein, } \\
\text { D3CUR [G] }\end{array}$ \\
\hline DL1GZ & DE3500 & Gerhard Keesdorf, Magdeburg Usedom [G] \\
\hline DL1HB [E] & DE3290 & Erwin Rüdiger, Frankfurt/M. Oberursel [G] \\
\hline DK3ZR & DE1864H & Werner Wille, Halle Köln Harburg Hanau Italien, D3DIH, DA3GB [G] \\
\hline DL1HF & DE2605J & Walter Falke (-1949), Warnemünde Limburg, D4CKJ [G] \\
\hline DL1HH [E] & DE3214 & Hermann Groh, Frankfurt/M Essen, DA3AQ [G] \\
\hline DL1HI & EK4KU & $(-1952)$ \\
\hline DL1HJ & DE2850N & Hans Burkhard, Neuhausen/Fildern, D3DHN [E], [G] \\
\hline DL1HK [E] & DE1733F & Erich Lehwald, Berlin-Tempelhof, D4CKF [E] D4CKF, D4HCF [G] \\
\hline DL1HL [E] & DA1GW & "Hans" Herbert Greiner, Nellingen/Fildern Tübingen [Schick, 2016] \\
\hline DL1HM[E] & DA1CC & Egon Koch, Stuttgart [E] \\
\hline DL1HN & DE6007 & “Rolf” Walter Kalb, Berlin Stuttgart Hamburg, DA1AZ [G] \\
\hline DL1HP & DE2262N & Kurt Leucht, Stuttgart, D3DDN [E], [G] \\
\hline DL1HQ & D4RDX & $(1915-1989)$ \\
\hline DL1HR [CB1951] & DE6748 & Martin Müller, Heilbronn, D4OLN [G] \\
\hline DL1HS [E] & DE3749 & Herbert Prottengeier, Stuttgart Ludwigsburg, DA1AT, OK8BB [G] \\
\hline DL1HT & DE5006N/ DE732 & $\begin{array}{l}26 \text { [E] “Joe” Wolbert Schneidewind, Stuttgart, DA1GN, member FWGM [G] } \\
\text { [Schick, 2016] }\end{array}$ \\
\hline DL1HTK [in 1991] & DE6555 & Gerhard Kloss, Halle/Saale [G] \\
\hline DL1HU & DE6022 & Werner Stockmayer, Stuttgart [G] \\
\hline DL1HX [E] & DE2421R & Wilhelm Wieland (-<1977), Würzburg Kirchheim Gschwend [G] \\
\hline DL1HZ & D4BTT & $(-\sim 1993)$ \\
\hline DL1IA $[\mathrm{E}]$ & DE1802 & Karl-Heinz Güttner, Zoppot Danzig/Langfuhr, YM4AD [E], YM4DSG [E], [G] \\
\hline DL1IB [E] & EK4ZO & YM4ZO, YM4AA (-1972) \\
\hline DL1IC & DE2428J & Karl Schwien (-1983), Pasewalk Schlamersdorf [G] \\
\hline DL1IE [E] & DE3890 & Karl Bergmann (-1983), Danzig Langfuhr Schladen/Harz Neumünster, YM4AU [G] \\
\hline DL1IF & DE3052 [E] & Bernhard Kabel, Neumünster, D4VZV, DA6BN [G] \\
\hline DL1IH & EK4DB & $(-1979)$ \\
\hline DL1IM [E] & DE2038V & Walter Peters (-1968), Schülp Scholderup Meldorf, D4WNV [E], [G] \\
\hline DL1IN [E] & D4MZF & $(-1970)$ \\
\hline DL1IP [E] & DE1761V & Detlev Mißfeld (-1976), Schleswig Flensburg Berlin [G] \\
\hline DL1IS & DE5065J & Adolf Behn, DE3352P, D4TZJ, member FWGM [G] \\
\hline DL1IT [E] & DE3982 & Dr. Günter Pfeffer, Dortmund, Nordstrand-Morsumhafen [G] \\
\hline DL1IW & DE6265 & Willi Petersen, Schleswig Nordstrand [G] \\
\hline DL1IX & DE6753 & Waldmar Kehler, Königsberg Husum, D3FBA [G] \\
\hline DL1JC [E] & DE0243 & Heinrich Lampe, Kiel [G] \\
\hline
\end{tabular}




\begin{tabular}{|c|c|c|}
\hline DL1JD $[\mathrm{E}]$ & DE1884V & Günter Camps, Kiel, DE5019, D4XDV, DA6AZ, member FWGM [G] \\
\hline DL1JF $[\mathrm{E}]$ & D4GCF(2) & $(->1995)$ \\
\hline DL1JG & EK4KH & $(-1987)$ \\
\hline DL1JH [E] & DE4071 DE4073 & Otto Schmieder, Kiel [G] \\
\hline DL1JJ $\quad[\mathrm{E}]$ & D4OMG & $(-\sim 1980)$ \\
\hline DL1JK $[\mathrm{E}]$ & D4IDF & $(-1987)$ \\
\hline DL1JL & D4MVR & [E:shack photo] \\
\hline DL1JM & DE3078 & “Henry” Rolf Hubbertz, Düsseldorf Krefeld, DA4DS [G] \\
\hline DL1JO & DE6055 & Josef Birker, Viersen [G] \\
\hline DL1JQ & DE6861 & Otto Liefering (-1971), Kettwig [G] \\
\hline DL1JT $[\mathrm{E}]$ & DE6549 & “Köbes” Hermann Ferring, Oberhausen Mülheim/Ruhr, DA4CA, DA4KA, [G] \\
\hline DL1JU [E] & EK4RY, EK4JU & $(-\sim 1975)$ \\
\hline DL1JW [E] & DE6271 & Hubert Esser, Kessenich Bad Kreuznach, DA4NI [G] \\
\hline DL1JX $[\mathrm{E}]$ & DE3930 & Theodor Greulsberg (-1984), Köln Junkersdorf, DA4NL [G] \\
\hline DL1JY $[\mathrm{E}]$ & DE3796 & Karl Krumbach (-1966), Düren Rösdorf, D4GQI, DA4ND [G] \\
\hline DL1KA & DE2742 & Paul Prost, Köln Frechen [G][E:shack photo] \\
\hline $\mathrm{DL} 1 \mathrm{~KB}[\mathrm{E}]$ & DE3191 & $\begin{array}{l}\text { "Jonny” Hans Pazem, Köln Rösrath, EZ4X } 1946 \text { [E], DA4NA, [G], } \\
\text { started } 1933 \text { [on } 1964 \text { QSL] }\end{array}$ \\
\hline $\mathrm{DL} 1 \mathrm{KC}[\mathrm{E}]$ & DE2284 & Heinz Salitz, Köln/Ehrenfeld, D4YHI, D4YFI, DA4NB, started 1934 [G] \\
\hline DL1KD & DE3013 & Friedrich Schmitz, Köln, D4FSI(2) [G] \\
\hline $\mathrm{DL} 1 \mathrm{KE}[\mathrm{E}]$ & DE6329 & Günter Burgbacher, Bremen [G] \\
\hline $\mathrm{DL} 1 \mathrm{KG}$ & DE2570K & Julius Felchner, Bremen Zeven, D4MAK [G] \\
\hline DL1KH $[\mathrm{E}]$ & DE2074K & Hans Goldmann (-1966), Bremen Marburg, D3AVK, DA5AV [G] \\
\hline DL1KJ & DE2056K & Ernst Holm, Bremen, DA5AW [G] \\
\hline DL1KK $[\mathrm{E}]$ & DE2568 & Hinrich Müller (-1972), Bremen-Oberneuland, D4YYK [E], [G] \\
\hline DL1KN & DE2063K & Bruno Wegener (-1961), Bremen [G] \\
\hline $\mathrm{DL} 1 \mathrm{KO}(1)$ & EK4PX & $(-1957)$ \\
\hline DL1KO(2) [CB1962] DL1LBI & $\mathrm{DE} 3543[\mathrm{E}]$ & Herbert Wulfhoop (- 1963), Stettin Köln Kiel, D4JTB [G] \\
\hline DL1KQ & DE3910 & Ernst Burghardt, Hannover Sarstedt, D4XGK, DA5BT [G] \\
\hline DL1KR & DE3634 & Erich Luehrs, Edewecht, D4YDK, DA5BR [G] \\
\hline DL1KU [E] & DE2671K & Bernhard Willers, Oldenburg, DA5WB [G] \\
\hline $\mathrm{DL} 1 \mathrm{KV}[\mathrm{E}]$ & DE6220K [E] & $\begin{array}{l}\text { Otfried Lührs, Göttingen Hamburg Wilhelmshaven, HK3AH, XE1KKV [E], } \\
\text { PA0KVN [E], [G] }\end{array}$ \\
\hline $\mathrm{DL} 1 \mathrm{KW}[\mathrm{E}]$ & EK4GB & $(1898-1980)$ \\
\hline $\mathrm{DL} 1 \mathrm{KX}[\mathrm{E}]$ & DE2052H & Berthold Dalmahay, Dortmund Nordhorn, D4RAH [G] \\
\hline $\mathrm{DL} 1 \mathrm{KZ}[\mathrm{E}]$ & DA5RK/DA5BE I & Hans Ulrich Schnacke, Leer [E] \\
\hline DL1LA & DE6896 & Bruno Specht, Ditzumerverlaat [G] \\
\hline DL1LB $[\mathrm{E}]$ & D4NBC & $(-1983)$ \\
\hline $\mathrm{DL} 1 \mathrm{KO}(2)$ & $\mathrm{DE} 3543[\mathrm{E}]$ & Herbert Wulfhoop (- 1963), Stettin Köln Kiel, D4JTB [G] \\
\hline DL1LC $[\mathrm{E}]$ & DE2233H & Hans Richarz, Norderney Island, DA5BF $[\mathrm{E}],[\mathrm{G}][\mathrm{E}]$ \\
\hline DL1LD $[\mathrm{E}]$ & EK4GR & $(1911-1991)$ \\
\hline DL1LJ [E] only [CB49/50] & DE6809 & Gert Lehmann, Fluyn/Moers [on QSL] Duisburg Essen, DA4JS [G][E] \\
\hline DL1LK & DE3784 & Ernst-Wilhelm Hans, Mönchengladbach [G] \\
\hline DL1LL & EK4QF & $(-1963)$ \\
\hline DL1LM & DE2167O & Fritz Kaeser (-1952), Weinheim Mannheim Ludwigshafen, D4SHO [G] \\
\hline DL1LN [CB1955] & EK40C(1) & $(-\sim 1984)$ \\
\hline DL1LP & EK4OD & $(-\sim 1976)$ \\
\hline DL1LQ [E] & DE2881O & Heinrich Muselmann (-1978), Mannheim, D4STO, DA1DC [G] \\
\hline DL1LU [E] & DE6692 & “Max” Rolf Huber, Esslingen, DA1GA [G][Schick, 2016] \\
\hline DM2ACM & EK4MX & $(->1994)$ \\
\hline DL1LX $[\mathrm{E}]$ & DE3567 & Georg Rentschler, Ulm Kissendorf [G] \\
\hline DL1LY $[\mathrm{E}]$ & DE1503N & Thomas Faul (->1992), Heidelberg, D4LDX [G] \\
\hline DL1LZ $[\mathrm{E}]$ & D4HBT & $(-2003)$ \\
\hline DL1MB OE5OW HB9ACS & DE6825 & “Kail” Karl Weinberger, Ulm Steir Zimikon, DA1CR [E], [G] \\
\hline $\mathrm{DL} 1 \mathrm{MC}[\mathrm{E}]$ & $\mathrm{DE} 3727[\mathrm{E}]$ & $\begin{array}{l}\text { “John” Johann Adolf Engelhardt, Neumark St. Veith Regensburg, D4SMP (unlis 1935), } \\
\text { D4SMR (unlis), DA2AX, DA2OZ. D4SMP was worked by W1ME in } 1935 \\
\text { [Radio 2/1936]. In } 1944 \text { he was incarcerated for illegal transmission [G][E] }\end{array}$ \\
\hline $\mathrm{DL} 1 \mathrm{MD}[\mathrm{E}]$ & DE6623 & Dr. med. Heribert Rechl, Weiden Regensburg Murnau, DA3HR [G][E] \\
\hline $\mathrm{DL} 1 \mathrm{ME}[\mathrm{E}]$ & DE3972 [E] & "Max" Raimund Kainer, Augsburg Göggingen, DA2CW [C], [E] \\
\hline $\mathrm{DL} 1 \mathrm{MF}[\mathrm{E}]$ & DE1747P & Georg Kirner, München, D4BMP, D4SPP [G] \\
\hline DL1MG & DE6189 & Hans Pagels, Neustettin Pensberg [G] \\
\hline DL1MI $[\mathrm{E}]$ & DE1925H & Dr. Hans Jürgen Twick-Hoenig, Mönchengladbach, D4VGH [G] \\
\hline $\mathrm{DL} 1 \mathrm{MK}[\mathrm{E}]$ & DE3154 & Friedrich Kaemper, Hagen, DA4SA [G] \\
\hline DL1ML $[\mathrm{E}]$ & DE3825 & Felix Hardt (->1980), Hagen Bonn, DA4SB [G] \\
\hline DL1MM(1) & DE3915, DE3951? & ? Hans Reuber, Hagen, DA4SR [G] \\
\hline
\end{tabular}




\begin{tabular}{|c|c|c|}
\hline $\operatorname{DL} 1 \mathrm{MM}(2)[\mathrm{E}][\mathrm{CB} 1965]$ & EK4JA & $(-2017)$ \\
\hline $\mathrm{DL} 1 \mathrm{MN}[\mathrm{E}]$ & DE6312 & Max Kulinski (Jonatan), Hagen, DA4SC [G] \\
\hline $\mathrm{DL} 1 \mathrm{MO}[\mathrm{E}]$ & DA4SS [E] & "Quax" Gerd Schreiber, Hagen [E] \\
\hline DL1MV & DE2294 & Ernst Blümel, Köln, started 1937 [G] \\
\hline DL1MW & D4LLL & $(-\sim 1961)$ \\
\hline DL1NA & DA4FL & Eberhard Görgens, Alsdorf near Aachen [E] \\
\hline DL1NB(1) [CB1951] & DE6697 & Willy Kattwinkel, Gummersbach [G] \\
\hline $\mathrm{DL} 1 \mathrm{NB}(2)[\mathrm{E}]$ & DE6617 & Hermann Josef Kauven, Aachen [G] \\
\hline $\mathrm{DL} 1 \mathrm{NC}[\mathrm{E}]$ & DE3912I [E] & Josef Knipprath, Köln Lendersdorf Düren Niedau, DA4ND [G] \\
\hline DL1NE & DE2555I [E] & Theodor Krath, Köln-Kalk Heiligenhaus über Overath [CB1951] \\
\hline DL1NF & DE6346 & Karl Langguth, AachenAhlsdorf, DA4ZR [G] \\
\hline DL1NG [E] & DE2504G & Karl-Heinz Lebrecht, Liegnitz Duisdorf-Bonn, D4KMG, DA4CZ [G] \\
\hline DL1NH [E] & EK4PV & $(-\sim 1990)$ \\
\hline $\operatorname{DL} 1 \mathrm{NM}(1)$ & DE6130 & Ferdinand Dormann, Marienberg Wiesbaden [G] \\
\hline DL1NM(2)[E] DC9NM & D4DVH & $(-\sim 1993)$ \\
\hline DL1NN & DE2807H [E] & Willi Hagedorn, Heiligenhaus Höxter, DA4SH [G] \\
\hline $\operatorname{DL} 1 \mathrm{NP}(1)[\mathrm{E}]$ & DE1988H & Emil Kockskämper, Witten-Annen, DA4YA [G] \\
\hline DL1NP(2)[1963] DL1GM & DE6807 & Jürgen Bennöhr (Ben), Kiel Hassee Parching Aumuehle Fahrdorf [G][E] \\
\hline DL1NQ & D4WSL & \\
\hline DL1NR(1) & DE3036 & Adolf Schmidt (-1981), Wanne-Eickel, DA4UL [G] \\
\hline DL1NR(2) (got call ca 1981-85) & EK4VD & $(1910-1987)$ \\
\hline DL1NU & EK4LX & $(-1982)$ \\
\hline DL1NV [E] & DE3037 & Wilhelm Wischeloh (-1987), Bochum [G] \\
\hline DL1NW[E] & DE6660 & Karl Zettler, Halle Hagen Hemer, DA4SR [E], [G][E] \\
\hline DL1NX [E] & DE3174 & Detlev Radbruch, Lübeck Kiel [G] \\
\hline DL1OB & D4LAC & $(->1995)$ \\
\hline DL1OD & $\mathrm{DE} 0829 \mathrm{H}[\mathrm{C}][\mathrm{E}]$ & Dr. Karl Ernst Wetzlar (-1974), Hagen Düsseldorf, DA5SW [G] \\
\hline DL1OE & EK4RU & $(-1983)$ \\
\hline DL1XP(2) & DE3808 & Heinz Harscheid (-1973), Wuppertal [G] \\
\hline DL1OG [E] & D4BWL & $(-1979)$ \\
\hline $\mathrm{DL} 1 \mathrm{OH}$ & EK4LY & $(-1969)$ \\
\hline DL2IT [E] & DE3812 & Dipl.-Ing K. H. Schmitter, Ismaning, DA4DS [E][G] \\
\hline DL1OL $[\mathrm{E}]$ & DE6222 & Karl Schroers, Krefeld, DA4ND, DA4DX [G] \\
\hline DL1OM [E] & DA4JC & Heinz Günter Happel (-2020), Mülheim [E] \\
\hline DL1OO $[\mathrm{E}]$ & DA3FU & Heinz Klauer, Frankfurt [DARC Hessen, 1997][E] \\
\hline DL1OS & DE3321 & Klaus Dubois, Andernach Mülheim Kamp Lintfort Welver [G] \\
\hline DL1OU [E] & DE7123 [E] & Erhard Müller, Frankfurt/M [E] \\
\hline DL1OV DM2ABC DM2ACA & EK4EY, EK4AC & EK4VS(2) \\
\hline DL1OW [E] & DE6031 & $\begin{array}{l}\text { Hans-J. Trappenberg, Bielefeld Langfeld Halle/W, D3DUH, D4ROK, DA4MM, DA4JJ } \\
{[\mathrm{G}][\mathrm{E}]}\end{array}$ \\
\hline DL1OX [E] & DE3991H [E] & Eduard Wacker, Warendorf/Ems Bottrop [E][G] \\
\hline DL1OY & DA4GG & Helmut Liebich, Marl [E] \\
\hline DL1OZ [E] & DE2741R & Johann Alfred Benker, Habnith/Ofr. Bielefeld München [G] \\
\hline DL1PA [E] & DE6492 & Ewald Wiethölter, Bielefeld, DA4JA [G] \\
\hline DL1PB & D4BNH & $(-1987)$ \\
\hline DL1PC & D4CAL(2) & $(-\sim 1975)$ \\
\hline DL1PE [E] & DE3138 & Karl Taddey (-1997), Velbert Fockbeck, DA4KT, [G] \\
\hline & & DL3RV Emil Karl Taddey at same address \\
\hline DL1PH & EK4US & 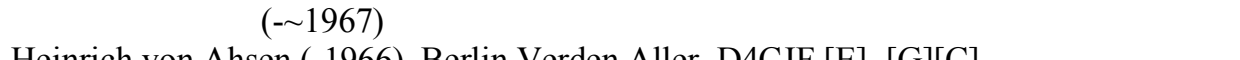 \\
\hline DL1PJ(1)[E] & DE1722 & Heinrich von Ahsen (-1966), Berlin Verden Aller, D4CJF [E], [G][C] \\
\hline DL1PJ(2) [RL77] & DE6693 & Dr. Johannes Lehmann (-1977), Velbert Wuppertal [G][E] \\
\hline DL1PK $[E]$ & DE6387 & Carl August Dittmers, Verden/Aller Celle Hamburg, D4PEJ [G] \\
\hline DL1PM $[\mathrm{E}]$ & DE3501 & Ernst Manske, Ratibor Frankenstein Hamm Wifelstede Hamburg, DA5BC [G] \\
\hline DL1PN $[\mathrm{E}]$ & DE2332G & Helmut Leder (-1967), Michelsdorf Einsal [G] \\
\hline DL1PP $[\mathrm{E}]$ & DE2116H & Erich Wrona, Dortmund Diel, D3BJK, D3BJV, DA5KB [G] \\
\hline DL1PQ [E] & DA6KG & Heinz Friedrich, Osnabrück [E] \\
\hline DL1PS [E] & EK4JK & $(1901->1994)$ \\
\hline DL1PV [E] & DE2025K & Hans Jürgen Schwartze, Osnabrück, D4SZK [G] \\
\hline DL1PZ & DE2450 & Walter Hentschel, Hamburg/Eidelstedt Hannover, D3BZK [G] \\
\hline DL1QA [E] & D4TEG, D4GSC & $(-1974)$ \\
\hline DL1QB & DE1473M & Dieter Schuricht, Dresden Bremen, D4LJM, D4MBM, D4BQM [G] \\
\hline DL1QC & DE6284 & Günter Sander, Köln Merheim [G] \\
\hline DL1QH & DE6442 & Wilhelm Kaiser, Bochum Bielefeld [G] \\
\hline DL1QN [E] & DE1506K & Herbert Dancker, Hannover Hamburg, D4BXK, D4JXK, D4BYJ, D4EDQ [G \\
\hline DL1QO [E] & DE1853 & Heinrich Fischvoigt (-1966), Hannover/Ricklingen, D3AUK [G] \\
\hline
\end{tabular}




\begin{tabular}{|c|c|c|c|}
\hline & D4MRJ & $(1913-1989)$ \\
\hline \multicolumn{2}{|l|}{ DL1QQ } & DE3747 & Willy Lehmensiek, Alfeld/Leine Fassberg, D3CNK, D4CND(2), DA5NF [G] \\
\hline \multicolumn{2}{|l|}{ DL1QR [E] } & EK4WK(2) & $(->1995)$ \\
\hline \multicolumn{2}{|l|}{ DL1QS [E] } & D4WQS & $(-1987)$ \\
\hline \multicolumn{2}{|l|}{ DL1QT [E] } & EK4KU & $(-1991)$ \\
\hline \multicolumn{2}{|l|}{ DL1QU } & DE3466 & Max Mathis, Ratibor Celle/Eschede, DA5BL, DA5HC [G] \\
\hline DL1QV & DL6QZ & DE6409 & Lothar Meuter, Danzig Gifhorn Regensburg, YM4BD, DA4BD [G] \\
\hline DL1QW[E] & DK4AUU & EK4AU & $(1912-1972)$ \\
\hline \multicolumn{2}{|l|}{ DL1QZ } & DE2204K & Alwin Düngefeldt, Celle [G] \\
\hline \multicolumn{2}{|l|}{ DL1RA [E] } & DE7379 & Heinz Bürger, Peine bei Hannover, DA5MB [E] \\
\hline \multicolumn{2}{|l|}{ DL1RB $[\mathrm{E}]$} & DE2476, DE327 & 8 Walter Leonhardt, Hannover, DA5CC? [G], started 1934 [QSL 1969, E] \\
\hline \multirow{2}{*}{\multicolumn{2}{|c|}{$\begin{array}{l}\text { DL1RC } \\
\text { DL1RD [-CB1958] }\end{array}$}} & DE2654F & Hans Bödeker (-1988), Berlin Rinteln Darmstadt [G] \\
\hline & & DE0088K & Hans Rundshagen (- 1958), Hannover Uelzen [G] \\
\hline \multicolumn{2}{|c|}{ DL1RE } & DE6168 & Hans Küster, Hannover [G] \\
\hline \multicolumn{2}{|l|}{ DL1RF [E] } & DE6457T [E] & Franz Kutschera (-1987), Magdeburg Hannover, DA5CI [G] \\
\hline \multicolumn{2}{|l|}{ DL1RH } & DE6569 & Hermann Iburg (-1990), Hannover München [G] \\
\hline \multicolumn{2}{|l|}{ DL1RI } & DE6626 [TUC] & Hans Grebenstein, Nürnberg [G] \\
\hline \multicolumn{2}{|l|}{ DL1RJ } & DE6077 & Karl-Heinz Buchsteiner, Königsberg Borghöfe [G] \\
\hline \multicolumn{2}{|c|}{ DL1RMA DM2AMM, Y21MM } & D4BMH & $(-2004)$ \\
\hline DL1RQ & & DE6659 & Horst Grasmann, Hannover [G] \\
\hline DL1RT & & DE2557 & Hans Kogel, Lockstedt Wohldorf, D3IUJ [G] \\
\hline DL1RU & & DE6666 & Dr. Heinrich Liedgens, Hamburg/Billstedt [G] \\
\hline DL1RW & & DE2362 & John Pramann, Hamburg Neuss, D4LCJ, DA6RS [G] \\
\hline DL1RX [E] & & EK4FJ & $(-1982)$ \\
\hline DL1RY & & DE1943H [E] & Ernst Schamann, Hamburg Berlin Wanne-Eickel, D3CFH [E],[G] \\
\hline DL1RZ & & DE2631J & Adolf Schütz, Hamburg/Stellingen Bahrenfeld, D4KHJ [G] \\
\hline DL1SA & & D4LBJ & $(-\sim 1975)$ \\
\hline DL1SC [E] & & D4RNX & $(-\sim 1985)$ \\
\hline DL1SD $[\mathrm{E}]$ & & DE6427 & Wolfgang Karl Wichmann, Hamburg/Altdamm [G] \\
\hline DL1SI & & EK4QQ & $(-1957)$ \\
\hline DL1SK $[E]$ & & DE6056 & Carl Feld (-1984), DA4XJ [G] \\
\hline DL1SL & & DE2412H & Heinz Hagehülsmann (-1982), Münster, DA4XB [G] \\
\hline DL1SM [E] & DL3FH & DA4BM & Wolfgang Molz, Diessen am Ammersee, VU2WM, G5AGI [E] \\
\hline DL1SN [1970] & DL1CO & DE6039 & Eugen Englert (-1990) \\
\hline DL1ST & & DE1673O & Rudolf Maushart (-1969), Hundseck/Schwarzwald, D4BQO [E], [G] \\
\hline DL1SU & & EK4FH & $(-1956)$ \\
\hline DL1SV [E] & & DE6777 [E] & Albrecht Heinrichs, Braunschweig [G] \\
\hline DL1SX [E] & & EK4QB & $(-\sim 1993)$ \\
\hline DL1SY. [E] & & DE8247 & Klaus-Dieter Möhle, Braunschweig Stuttgart [E] \\
\hline DL1TA & & $\mathrm{DE} 0110 \mathrm{D} / \mathrm{C}$ & Henry Meyer (-1955), Braunschweig [G] \\
\hline DL1TB [E] & & DE6197 & Horst Capptuller, Braunschweig Berlin, DA5IC [G] \\
\hline DL1TC & & DE2275D & Helmut Brockmann, Braunschweig [G] \\
\hline DL1TH $[\mathrm{E}]$ & & DE1797G & $\begin{array}{l}\text { "Haro" Helmut Nabroth, Berlin Breslau Göttingen-Grone, D4XHG [CQ 6/1937], } \\
{[\text { E][G] }}\end{array}$ \\
\hline DL1TJ & & EK4PI & $(-1980)$ \\
\hline DL1TL $[\mathrm{E}]$ & & $\begin{array}{l}\text { DE0826/ } \\
\text { DE6126 }\end{array}$ & $\begin{array}{l}\text { Alfons Dorlars, Oberhausen Göttingen Leverkusen Hamm Bad Pyrmont, } \\
\text { DA4TA, DA4WW, DA5ZO [G] }\end{array}$ \\
\hline DL1TM [E] & & DE2080K & Heinrich Fricke, Hann. Münden Bonafort, D4UWD [G] \\
\hline DL1TN & & $\mathrm{DE} 1517 \mathrm{D}[\mathrm{E}]$ & $\begin{array}{l}\text { Kurt Fürchtenicht (-1977), Berlin Pforzheim Göttingen, D4MPP, D4BPK, D4FYF, } \\
\text { D4FYD [G] }\end{array}$ \\
\hline DL1TO [E] & & DE3058 & Helmut Grupe (-1985), Bochum [G] \\
\hline DL1TP [E] & & DE2970D/W & Reinhold Wrede (-1975), Thiede bei Braunschweig, DA5JA [G][E] \\
\hline DL1TQ [E] & & DE6865 & Joachim Oswald, Göttingen Dramfeld, DA5HZ [G] \\
\hline DL1TR $[\mathrm{E}]$ & & DE2525D & Friedrich Haensch (-1978), Göttingen, D4LVF, D4UYD [E], DA3ZZ [G] \\
\hline DL1TS & & DE2217J & Karl Peters, Kehl/Rh. Holzminden Hildesheim Hannover, D3BHK, DA5CC? [G] \\
\hline DL1TT & & DE3250 & Fritz Mushake, Kittlitz/Löbau/Sachsen, D4QQM, D4YXM [G] \\
\hline DL1TU [E] & & EK4BM & $(-1962)$ \\
\hline DL1TV $[\mathrm{E}]$ & & DE8156 [E] & Gerhard Zickwolff, Volzum Hamburg [E] \\
\hline DL1TZ & & D4BWH(1) & $(->1995)$ \\
\hline DL1UB & & DE3987 & Gerhard Harm, Babelsberg Karlsruhe [G] \\
\hline DL1UD(1) [CB1 & 953] & DA5MF & Gunter Seidenberg, Bremen [J:14] \\
\hline $\operatorname{DL} 1 \mathrm{UD}(2)[\mathrm{CB} 1$ & 957] & EK4UU(1) & $(-\sim 1957)$ \\
\hline DL1UE [E] & & EK4BE & $(->1994)$ \\
\hline DL1UH & & D4RPV & $(->1995)$ \\
\hline DL1UK & & DE2820N & Erwin Kübler (-1979), Wuppertal-Barmen, \\
\hline
\end{tabular}




\begin{tabular}{|c|c|c|c|}
\hline DL1UL & DL3ZY? & D4LCJ & $(-\sim 1984)$ \\
\hline DL1UM [E] & & DE1936C & Walter Plage, Beelitz Darmstadt, DA6RX [G] \\
\hline DL1UN & & DE3071 & Willi Witzke, Bunslau Elmshorn [G] \\
\hline DL1US [E] & [CB1965] & DE3549 & Heinz Nolte, Düsseldorf-Gerresheim, D4VHH, D4VHM [G] \\
\hline DL1UU [E] & & D4BIT & $(-1991)$ \\
\hline DL1UX [E] & & DE2532J & Rolf Netzband, Hamburg, D4AAJ, DA6HY [G] \\
\hline DL1UY & & DE6249 & “Karl” Karl Ranke, DA6KB [QSO with DA6LI, E] Hamburg [G] \\
\hline DL1UZ [E] & & DE1917 & $\begin{array}{l}\text { Claus Mohr, Düneberg/Elbe Geesthacht near Hamburg, D4GWJ [E], DA6GW } \\
\text { on } 1951 \text { QSL changed name to Claus Sterz [G] }\end{array}$ \\
\hline DL1VA & & DE2429J & Werner Weigardt, Hamburg/Bergedorf [G] \\
\hline DL1VB [E] & & EK4HY & $(-\sim 1956)$ \\
\hline DL1VC $[\mathrm{E}]$ & & DE1675V & $\begin{array}{l}\text { Herbert Klinkert, Dresden Langenhagen Hannover Bissendorf Berlin/Tempelhof Kiel, } \\
\text { D4QIV [CB1937], [G][E] }\end{array}$ \\
\hline DL1VE [E] & & EK4QK & $(-1963)$ \\
\hline DL1VF & & EK4TC & $(-\sim 1958)$ \\
\hline DL1VG [CB19. & 51-1953] & DE1075 & Hermann Himmler, XDE1075 [E, QSL 1930], Swinemünde Stettin München [E] \\
\hline DL1VG $[\mathrm{E}, \mathrm{QS}$ & L 1963] & EK4GG & $(-\sim 1964)$ \\
\hline DL1VH & & DE3839 & Willibald Vogt, München [G] \\
\hline DL1VI & & DE7010 & August Weber, München [G] \\
\hline DL1VK & & DE3778 & Alfred Eschenbacher, Augsburg [G] \\
\hline DL1VM[E] & & DE3434 & Adolf Mehling, München, DA2PM [G][E] \\
\hline DL1VN [E] & & DE3969 & Hans Schrödel, Augsburg, DA2HF [G] \\
\hline DL1VP [E] & & DA2AL,DA2AP & Karl Wunschel, Unterhaching bei München, Ottobrunn, D3CAP, D3JYP [E] \\
\hline DL1VQ [E] & & DE3342 & Kurt Ladwig (-1986), Essen Frankfurt/M. München Bad Wiessee/Tegernsee [G][E] \\
\hline DL1VR $[E]$ & & EK4GB & $(-\sim 1993)$ \\
\hline DL1VS [RL197 & 78] DL7AV & DE6200 & Eduard Voigt \\
\hline DL1VT & & $\mathrm{DE} 1669 \mathrm{~J} / \mathrm{Y}[\mathrm{E}]$ & Werner Haage (-1955), Danzig-Langfuhr Glonn München, YM4AC, YM4DSH [E],[G] \\
\hline DL1VU & & DE6909 & Karl-Heinz Hille, Thonsdorf Lengries, DA2ZN [G] \\
\hline DL1VV [E] & & $\mathrm{DE} 1872 \mathrm{U}[\mathrm{E}]$ & $\begin{array}{l}\text { Ing. Max Transchel, Zwenkau/Leipzig Oberaudorf Hohenaschau, D3BWU [E], } \\
\text { DA2AG [E], [G] }\end{array}$ \\
\hline DL1VY [E] & & DE6285 & Werner Gornig, München/Puchheim [G] \\
\hline DL1WA[E] & & EK4FR & (1894-1973) \\
\hline DL1WH & & D4CKK & $(-\sim 1984)$ \\
\hline DL1WJ [E] & & DE2581 & Hilarius Decker, Köln Büchel bei Loope, since 1937 [G] \\
\hline DL1WK[E] & & EK4LA & $(-1974)$ \\
\hline DL1WL & & D4GPF & $(-1991)$ \\
\hline DL1WM & & DE2284 & Heinz Salitz, Köln/Ehrenfeld, D4YHI, D4YFI, DA4NB [G] \\
\hline DL1WN & & EK4WL & $(-\sim 1970)$ \\
\hline DL1WO & & DE6634 & Anton Jaust (-1977), Brühl Köln [G] \\
\hline DL1WQ & & DE2740 & Hermann Pahnke (-1973), Köln, D3FQI [G] \\
\hline DL1WR & & DE3949I [E] & Johann Rieke, Köln [G] \\
\hline DL1WU & & DE2220I & Kurt Wallenfang, Köln/Porz, D4YGI [G] \\
\hline DL1WV & & EK4DP & $(->1994)$ \\
\hline DL1WZ [E] & & DE1823K & W. Ludwig Wagner, Osnabrück, D4SYK [E], [G] \\
\hline DL1XA [E] & & Ritz & \\
\hline DL1XB [CB51 & ] DL1BW & DE3736 & Eugen Wachholz, Bremerhaven Wesermünde, DA5XR [G] \\
\hline DL1XD & & DE3175 & Heinz Hurrelmann, Einswarden Nordenham, D4ZYK, DA5XM [G] \\
\hline DL1XF & & EK4CR & $(-1953)$ \\
\hline DL1XH [E] & & DE6611 & Herbert Nitsch (Harry) (-1975), Memmingerberg Berlin Ulm, DL8EAQ [E, 1947] [G] \\
\hline DL1XM & & D4CCJ & $(-\sim 1993)$ \\
\hline DL1XP(1) [E] & & EK4NG & $(-\sim 1967)$ \\
\hline $\operatorname{DL} 1 X P(2)$ & DL1OF & DE3808 & $(-1973)$ \\
\hline $\operatorname{DL} 1 \mathrm{XR}(1)[\mathrm{E}]$ & & DE3624 & Helmuth Stübing (-1950), Gerswalde Templin Berlin Markt Grafling [G] \\
\hline $\operatorname{DL} 1 X R(2)$ & & DE1819F & Bruno Becker, Berlin/Schmargendorf, D4SUF [G] \\
\hline DL1XS [E] & & D4BRP & $(-\sim 1975)$ \\
\hline DL1XT [E] & & DE3986 & Kurt Loos, München, DA2CC [K], [G] \\
\hline DL1XV & & DE1550F & Herbert Wegener, Berlin-Friedrichsfelde München Pelzerhaken, D4GXF [E], [G] \\
\hline DL1XW[E] & & DE3402P [E] & Arnold Imberger (-1971), München [G] \\
\hline DL1XX [E] & W2HIU W2TO & DE6347 & Hans Meurer (-2007), Detmold Ridgewood, president NJDXA [G] \\
\hline DL1XY [E] & & DE1835R & Richard Marschall (-1982), Bad Neustadt/Saale Kleinwenkheim, D4SXR [G] \\
\hline DL1XZ $[\mathrm{E}]$ & & DE2737R & Adam Müller, Erlangen, D3GHR, DA2AZ [G] \\
\hline DL1YA [E] & & EK4KQF & \\
\hline DL1YB $[\mathrm{E}]$ & & $\mathrm{DE} 2544 \mathrm{~F}$ & $\begin{array}{l}\text { Werner Schöning, Feuchtwangen Berlin-Lichterfelde Schleswig, D4FOF, D3AZV, } \\
\text { DA2WS [G] }\end{array}$ \\
\hline DL1YC [E] & & EK4CO & $(-1998)$ \\
\hline
\end{tabular}




\begin{tabular}{|c|c|c|}
\hline DL1YD & DE0478R & Wilhelm Siebentritt (-1974), Vechta Nürnberg [G] \\
\hline DL1YE(1) & $\mathrm{DE} 3765[\mathrm{E}]$ & Michael Stuber, Nürnberg [E] QRT 6/1964 [G] \\
\hline DL1YE(2) [RL1977] & DE7006 & Gerhard Hagenow, Berlin Emden [G] \\
\hline DL1YF & DE3096R [E] & Fritz Scheiderer, Würzburg [G] \\
\hline DL1YN & $\mathrm{DE} 3022[\mathrm{E}]$ & $\begin{array}{l}\text { Johann Ediger jr., Berlin Lößnitz Jackson MI, D4PNF?, KA8OSS [CB1993], } \\
\text { LU3DVF [CB1962], [G][TUC] }\end{array}$ \\
\hline DL1YP $[\mathrm{E}]$ & DE6078 & Siegfried Fuhrmann, Königsberg Hamburg, started radio in 1920? [G] \\
\hline DL1YQ $[\mathrm{E}]$ & DA6AJ $[E]$ & Alfred J. Haberstein, Cuxhaven Danzig, DA6TX [E] \\
\hline DL1YR & DE2364K & Walter Hermann, Cuxhaven [G] \\
\hline DL1YS & D4IPF & $(-\sim 1955)$ \\
\hline DL1YT & DE8423 & Gerhard Hundt, Lübeck [E] \\
\hline DL1YU & EK4KQ & $(-1961)$ \\
\hline DL1YV & DE1558H & Helmut Jäger (-1985), Dresden Duisburg, D4LUH? [G] \\
\hline $\mathrm{DL} 1 \mathrm{ZC}[\mathrm{E}]$ & DE3172 & Julius Langhals, Cuxhaven [G] \\
\hline DL1ZE & EK4LV & $(1904-1974)$ \\
\hline DL1ZF & DE2585J & Friedrich Papius (-1970), Stade Lintig Bremerhaven/Schleuse, D3IRJ [G] \\
\hline DL1ZH & D4ZAR & $(-\sim 1984)$ \\
\hline DL1ZI［RL1977] & DE6878 & Josef Huber, München Ottobrunn Zorneding [G] \\
\hline DL1ZJ & DE6426 & Ernst Senkowski, Hamburg Mainz [G] \\
\hline DL1ZL $[\mathrm{E}]$ & DE6494 & Werner Trenkner, Lübeck [G] \\
\hline DL1ZM & DE2531J & Horst Vorrath, Hamburg [G] \\
\hline DL1ZN [E] & DA6WW & “Hans" Uwe Wolf, Cuxhaven/Sahlenburg, D4FOX [E] \\
\hline DL1ZQ [E] & DE6082 & Hans Dreyer, Walldorf Hamburg [G] \\
\hline DL1ZU $[\mathrm{E}]$ & EK4FA, EK4TB & $(-1985)$ \\
\hline DL1ZV & DE3797 & Alfred Zeller (-1974), Konstanz Waldshut am Hochrhein Offenburg, D4RHO [G] \\
\hline DL1ZW & DE6187 & Robert Wahrlich, Hamburg Halstenbeck (ausgeschieden 1969) [G] \\
\hline DL1ZX & D4POB & $(-\sim 1970)$ \\
\hline DL1ZY & DE3504 & Reinhold Wittrock, Quickborn Hamburg [G] \\
\hline DL2DZL Y24ZL DM2DZL & D4FRG(1) & $(1910-2008)$ \\
\hline DL2FF & DE3682 & Karl-Heinz Tiedtke, Rendsburg Kassel [G] \\
\hline DL2IR [CB1972] & DE6333 [E] & Kurt Schellschläger (- 1975), Weimar Winnert/Husum, DK8WD [G][E] \\
\hline DL2IT $[\mathrm{E}] \quad$ DL1OK & DE3812 & $\begin{array}{l}\text { Dipl.-Ing Karl-Heinz Schmitter (Klein-Schmitter -before 1939), Ismaning, DA4DS } \\
\text { [E][G] }\end{array}$ \\
\hline DL2IY $[\mathrm{E}]$ & DE0567 & A. C. Wilberforce, Köln/Bayerthal [G], D2IY \\
\hline DL2PE & EK4UAO & $(1895-1977)$ \\
\hline DL2TJ $[\mathrm{E}]$ & DE3329U & Kurt Unger, Chemnitz Hohndorf Düsseldorf, [G][CQ-MB 1/1939 p16] \\
\hline DL2WE $[E]$ & EK4GW & $(1904-1984)$ \\
\hline $\mathrm{DL} 2 \mathrm{ZO}[\mathrm{E}]$ & EK4SAR(3) & $(-1976)$ \\
\hline DL3AB $[\mathrm{E}]$ & DE7191 [E] & "Mac" Adolf H. Bott, Frankfurt/M, DA3AB [E], [K], [G][E] \\
\hline $\mathrm{DL} 3 \mathrm{AC}[\mathrm{E}]$ & $\mathrm{DE} 8319[\mathrm{E}]$ & Walter Gloger, Bad Nauheim [E] \\
\hline DL3AD $[\mathrm{E}]$ & DE6076 & Wilhelm Willi Bauer, Frankfurt/M, DA3KW [G] \\
\hline DL3AF $[\mathrm{E}]$ & DE3481 & Otto Feiler (-1966), Nürtingen [G] \\
\hline DL3AG & DE6276 & Erich Franz (-1986), Leipzig Bad Homburg [G] \\
\hline DL3AN & DE6662 & Wilhelm Scheuerle, Neuffen, DA1CD [G] \\
\hline $\mathrm{DL} 3 \mathrm{AO}[\mathrm{E}]$ & DE8395 & Dr. Rolf Schick, Esslingen Wolfschlugen, took DE test end of 1947 [E][Schick 2016] \\
\hline DL3AP & EK4XV & $(-\sim 1965)$ \\
\hline DL3AU & DE6365 & Ernst Mehtretter (-1985), Wesel Detmold, DA4AU [G] \\
\hline DL3AW [CB1951] & DE6702 & Dr. Horst Kortemeier, Herford [G] \\
\hline DL3AX $[\mathrm{E}]$ & DE6925 & Josef John, Neuenkirchen EckersdorfUnteraltsteinach Rheine, DA4ZJ [G] \\
\hline DL3AY $[\mathrm{E}]$ & EK4MA & $(-1976)$ \\
\hline DL3AZ $[\mathrm{E}]$ & EK4RD & $(-1972)$ \\
\hline DL3BA $[\mathrm{E}]$ & DA5XG & Günter Malchau, Bremerhaven [E] \\
\hline DL3BB & DE6675 & Kuno Radius, Aachen [G] \\
\hline DL3BJ $[\mathrm{E}]$ & DE8040 & "Joe" Jupp Kruse, Paderborn [E] \\
\hline DL3BO $[\mathrm{E}]$ & DE6364 & Hans Westerfeld (-1978), Nienburg/Weser, DA5QA [G] \\
\hline DL3BQ & DE6616 & Karl Heinz Claus (-1963), Celle, DA5AH [G] \\
\hline DL3BS $[\mathrm{E}]$ & DE8473 & Werner Boljahn, Mönchen-Gladbach [E] \\
\hline DL3BT & DE6747 & Herbert Trommel (-1976), Hannover [G] \\
\hline DL3BW & EK4PT & $(-1989)$ \\
\hline DL3CA $[E]$ & DE8853 & Karl-Heinz Jordan, Hildesheim [E] \\
\hline DL3CE & EK4AF(1) & $(-1989)$ \\
\hline $\mathrm{DL} 3 \mathrm{CF}[\mathrm{E}]$ & DA6YA & Günter Witt, Eutin [E] \\
\hline DL3CG $[\mathrm{E}]$ & $\mathrm{DE} 3542[\mathrm{E}]$ & Hans-Erich Reinke, Stettin Rendsburg Lübeck [G] \\
\hline DL3CJ & DE6578 & Ernst Reese, Kiel Holtenau [G] \\
\hline DL3CL & DE3932 & Heinz Gesch, Hamburg Quickborn [G] \\
\hline
\end{tabular}




\begin{tabular}{|c|c|c|}
\hline DL3CO [E] & DE2209J & Günther Merten (-2012), Cuxhaven, DE5034, D4QWJ [E], member FWGM [G] \\
\hline DL3CQ & DE6548 & Hans Pattberg, Bützfleh [G] \\
\hline DL3CS & DE7093 & Rudolf Staritz, Falkenstein Patzeried Berlin [G], author of [Staritz, 1985] \\
\hline DL3CT $[\mathrm{E}]$ & DE3388 & “Bobby” Fred Müller, Heidelberg Mannheim, D4BUS, DA1DM [G] \\
\hline DL3CU $[E]$ & DA4AC & Hubert Rosskamp, Uebach near Aachen [E] \\
\hline DL3CV & DE3011T & Rudolf Arnold, Arheilgen bei Darmstadt, DE3011T [E], [CB 1957] \\
\hline DL3CW [E] & EK4LF & $(->1994)$ \\
\hline DL3CY [E] & DE2247T & Hans Schnatz (-1973), Darmstadt [G] \\
\hline DL3DC $[\mathrm{E}]$ & EK4RC & $(-1999)$ \\
\hline DL3DD & DE6491 & Dr. Leopold Pomp (-2017), Mönchengladbach Frauenberg, DA3VE [G] \\
\hline DL3DE & DE2416H & Otto Hahn, Lignitz, DE2516G, D4IG?, DA3FD [G] \\
\hline DL3DF $[\mathrm{E}]$ & DE1516L [TUC] & Erich Kuhn, Halle Wiesbaden, D4MRL, D4BKL [G] \\
\hline DL3DJ & DE3785 & Erwin Meichert, Mönchengladbach [G] \\
\hline DL3DL & DE1524T & Heinz Weicker, Frankfurt/Main, D4SCT, D4BST, D4PTK [G] \\
\hline DL3DN & DE1684C & Werner Kobold (-1956), Königsberg Wiesbaden Neubabelsberg, D3DLC [E], [G] \\
\hline DL3DO $[\mathrm{E}]$ & DE6134 & $\begin{array}{l}\text { Albert W. Heine, Cottbus Konstanz Frankfurt München Lindau, D4FBC?, DK7CN, } \\
\text { DA2LY, DA1LY [G] }\end{array}$ \\
\hline DL3DQ(1) & EK4KMА & $(-\sim 1950)$ \\
\hline $\operatorname{DL} 3 \mathrm{DQ}(2)[\mathrm{E}]$ & D4GRW & $(-\sim 1968)$ \\
\hline DL3DR & DE6116 & Ludwig Delhees, Frankfurt/M [G] \\
\hline DL3DS & DE6910 & Karl-Heinz Claar $(-<1972)$, Niederrad bei Frankfurt [G] \\
\hline DL3DU $[E]$ & D4FCF & $(-\sim 1984)$ \\
\hline DL3DW $[E]$ & EK4HN & $(->1994)$ \\
\hline DL3DX & DE3947 & Hans Könemann, Hannover, DA4PD [G] \\
\hline DL3DY & DE2586J & Wilhelm Sauerland (-1964), Hamburg Bad Pyrmont, D3BPJ [G] \\
\hline DL3DZ & EK4HI & $(-1958)$ \\
\hline DL3EJ & DE6382 & Willi Klauser, Stuttgart [G][E] \\
\hline DL3EM [E] & DE3200 & Hubert Maier (-2003), Senden/Iller Ulm [G] \\
\hline DL3EN [E] & DE8396 & Adolf Maurer, Esslingen [E] \\
\hline DL3EP & DE3206 & Hermann Hartmann (-1983), Solingen Remscheid [G] \\
\hline DL3ET & DE3178, DE3218 & 8 “Kalle” August Ströhlein, Hamburg Esslingen, DA1GG [G][Schick, 2016] \\
\hline DL3EW [E] & $\mathrm{DE} 2448 \mathrm{~T} / \mathrm{R}$ & Pichard Paul Old (-1972), Darmstadt Arheilgen Straubing [G] \\
\hline DL3EY & EK4AM & $(-\sim 1960)$ \\
\hline DL3EZ & EK4AFM & $(-\sim 1956)$ \\
\hline DL3FB $[\mathrm{E}]$ & DE3485 & Wolfgang Berndts, Wuppertal Oerlinghausen [G] \\
\hline DL3FF & DE3303 & Karl Hahn, Hildesheim [G] \\
\hline DL3FH $[\mathrm{E}]$ & DA4BM & Wolfgang Molz, Diessen am Ammersee, VU2WM, G5AGI [E] \\
\hline DL3FK $[\mathrm{E}]$ & DE3989 & Hans-Joachim Klebig, Nowawes Solingen [G] \\
\hline DL3FL [E] & DA4ZC & Kurt Niepenberg, Haan [E] \\
\hline DL3FM [E] & DA3JD, DA4JD & “"Ernest”" Karl Gerhard Lickfeld, Mülheim [E] \\
\hline DL3FP & $\operatorname{EK4RR(2)~}$ & $(->1995)$ \\
\hline DL3FR $[\mathrm{E}]$ & $\mathrm{DA} 4 \mathrm{ZH}$ & Hans Gilfert, Essen [E] \\
\hline DL3FS & DE3838 & Gustav Nüttel (-1968), Essen Velbert [G] \\
\hline DL3FX & DE6057 & Erwin Urban (-1970), Schleswig [G] \\
\hline DL3FZ & DE1905R & Philipp Aumer (-1961), Nürnberg-Ost, D4STR(2) [E], [G] \\
\hline DL3GA & D4000 $(2)$ & $(->1995)$ \\
\hline DL3GC & DE2494C & Artur Herzog, Frankfurt/Oder Brandenburg Halle Nürnberg, DK8FZ [G] \\
\hline DL3GD & D4KQM & $(-\sim 1956)$ \\
\hline DL3GH & D4BGR & $(->1995)$ \\
\hline DL3GI. [E][CB51] & DE2291 & Erich Heine (-1980), Engelskirchen Bad Godesberg, D4YFI [G] \\
\hline DL3GK $[\mathrm{E}]$ & DE3493 & Baron von Sobeck Werder, Potsdam Unkel Heister Bad Godesberg [G] \\
\hline DL3GL $[\mathrm{E}]$ & DE6994 & Wolfgang Gruhle, Bonn, DA1DE, DA4DE [E] \\
\hline DL3GM & DE1957H $[\mathrm{E}]$ & Heinrich Valentin Pitthan jr., Köln/Brück Bonn Kohlkaul, D4YPI [G] \\
\hline DL3GP $[\mathrm{E}]$ & DE7009 & Kuno Halfen, Hennef Hellenthal [G] \\
\hline DL3GR & DE1442 & Norbert Hisserich, Wilhelmshafen [G] \\
\hline DL3GX & DE3775 & Otto Ammer, Augsburg [G] \\
\hline DL3GY & $\operatorname{D4SKO}(2)$ & $(-\sim 1955)$ \\
\hline DL3HB & DE3975 & Max Menke (-1979), Augsburg, DA2WC [G] \\
\hline DL3HE & DE6883 & Thomas Söldner (-1989), Berlin München [G] \\
\hline DL3HG [E] & DE6696 & Herbert Stotz, München [G] \\
\hline DL3HJ $[\mathrm{E}]$ & DE8964 & Karlheinz Dümmer, Emden [E] \\
\hline DL3HK [E] & DE2384 & Hugo Meyer, Flensburg Norderney, DA4NB [G] \\
\hline DL3HM & $\mathrm{DE} 1682 \mathrm{~J} / \mathrm{F}$ & Dietrich Merrem, Hamburg/Altona Berlin, D4BIF, D4GHJ [G] \\
\hline DL3HP & DE6103 & Emil Schäfer, Meppen [G] \\
\hline DL3HS [E] & DE1778F & Werner Bartolein, Berlin Ulm Blaubeuren [G] \\
\hline
\end{tabular}




\begin{tabular}{|c|c|c|}
\hline DL3HT & DE2014 & Josef Bayer, Nürnberg Halfing Kaufbeuren, left DARC in 1953 [G] \\
\hline DL3HU & DE1938P & Hans Bauer, München [G] \\
\hline DL3HW[E] & DE2614P & Hermann Hollenburger (-1979), München [G] \\
\hline DL3HX & DE5076P & Franz König, member FWGM [G] \\
\hline DL3IE [E] & DE3842 & "Bill” Willi Assold, München [G] \\
\hline DL3IO $[\mathrm{E}]$ & DE6720 & Hans Schulze-Allen, Soest San Jose Costa Rica [G] \\
\hline DL3IP & DE3888 & Hans Klostermann, Danzig Oliva Wildeshausen, YM4AT [G] \\
\hline DL3IQ [E] & DE3589 DE5014 & Herbert Hoffmann, Bochum-Harpen, D3JCH, member FWGM [G][E] \\
\hline DL3IV [E] & DE9210 & Arno Kirchner, Kirchheim [E] \\
\hline DL3JE [E] & DE1805F & $\begin{array}{l}\text { Werner Feilhauer (1902-1990), Breslau Danzig Berlin Göttingen München, D4CLK, } \\
\text { D3DZF, DA5ZN [G][OOTC] }\end{array}$ \\
\hline DL3JE(2)? & DE2502U & Kurt Zippert, Wurzen Jüterbog Berlin, D4NXB, D4CEL, started 1934 [G] \\
\hline DL3JG & EK4RM & $(-1956 / 57)$ \\
\hline DL3JH $[\mathrm{E}]$ & DE6383 & $\begin{array}{l}\text { Erich Köhler (-1985), Schloss-Hotel Grüner Wald, Wiesbaden Bad Homburg, DA3BB } \\
\text { [G] }\end{array}$ \\
\hline DL3JJ & D4AHQ & $(-1966)$ \\
\hline DL3JK [E] & DE2778T & Bernhard Tillmann, Gustavburg [G] \\
\hline DL3JL & DE6434 [E] & Fritz Eberhardt, Giessen Eulenloh Wunsiedel [G] \\
\hline DL3JZ & DE6095 & Walter Neuser, Frankfurt/M Bad Vilbel [G] \\
\hline DL3KO & DE1238J [E] & Rudolf Fehres (-1976), Arosa (Schweiz) Hamburg Wien [G][E] \\
\hline DL3KT [E] & D4BGH & $(-\sim 1993)$ \\
\hline DL3KU & DE2015R [E] & Georg Hagen (-1962), Bamberg [G] \\
\hline DL3KX & DE2943 & Paul Hiemisch (-1970), Bamberg [G] \\
\hline DL3LA & DE2520K & Dr. Hans Georg Korb, Bayreuth, DA3LA [G] \\
\hline DL3LB & DE1493R & Eduard Kühnle, Hamburg Bamberg, D4BMR [E], D4NZR [G] \\
\hline DL3LE(1) & DE3127 & Alfred Schwarz (-1957), Bitterfeld Fürth [G] \\
\hline DL3LE(2)[E] & DE2170G & Friedrich Nerlich, Breslau Kirchentumbach [G] \\
\hline DL3LI & DE9043 & Georg Zapf, Worzeldorf [E] \\
\hline DL3LJ $[\mathrm{E}]$ & D4GLD & \\
\hline DL3LK [E] & DK8AU & Fritz Strüber, Wallenstedt Sylt [E] \\
\hline DL3LR [E] & DE6414 & Kurt Frenz, Bad Bevensen, DA5KF [G] \\
\hline DL3LW & EK4QU & $(-1983)$ \\
\hline DL3MCF & EK4UF & $(1907-1996)$ \\
\hline DL3MCU & DE6649 & Winfried Hempel, Gotha [G] \\
\hline DL3MD & DE3507 DE4089 & Karl-Georg Kösel, Hannover Bad Godesberg [G] \\
\hline DL3ME [E] & EK4AT & $(1911-2002)$ \\
\hline DK4OG [RL77] & DE6386 & Kurt Gubbe, Stettin Hannover Bevensen [G] \\
\hline DL3ML & DA5FC & Rudolf Meier, Uelzen [J:14] \\
\hline DL3MQ & DE3548 & Helmut Lösche, Münster [G] \\
\hline DL3MS & DE6700 & Hans Kostmann, Stettin Wuppertal [G] \\
\hline DL3NJ(1) [CB1951] & DE6744 & Karl Bauer, Berlin Ladenburg, emigrated to the US [G] \\
\hline $\operatorname{DL} 3 \mathrm{NJ}(2)[\mathrm{E}]$ & DE3439 & Wilhelm Herbst, Wuppertal [G] \\
\hline DL3NU & DE6159 & Heinz Schlichting (-1971), Heidelberg Karlsruhe [G] \\
\hline OA4FN,CE3TE & DE1970F & Felix Cremers (-1968), Berlin/Lankwitz Lima [G] \\
\hline DL3NX [E] & DE2208J & $\begin{array}{l}\text { Willi Fock (-1967), Walsrode Rendsburg Cuxhaven Neuenkirchen, DE5064, } \\
\text { D4TPJ [E], member FWGM [G] }\end{array}$ \\
\hline DL3OK [E] [CB1949-1962] & DE9159 & Dr. med. Johannes Andresen, Flensburg, [E][DARC M03 History page] \\
\hline DL3ON & D4BWC & $(-\sim 1958)$ \\
\hline DL3OP & D4EJT & $(-\sim 1954)$ \\
\hline DL3OR? & D4KAN & \\
\hline DL3OR(2) & DE1928V & Walter Rieckermann, Kiel [G] \\
\hline DL3OX $[\mathrm{E}]$ & DE9364 & Manfred Harsdorff, Raisdorf near Kiel [E] \\
\hline DL3OY & $\mathrm{DE} 1054 \mathrm{O}[\mathrm{E}]$ & “Johann” Hans-Jürgen Franz (- 1952), Mannheim Heidenheim/Brenz [G][E] \\
\hline DL3PK & EK4UAF & $(-\sim 1983)$ \\
\hline DL3PM & DE3064 & Franz Czarnowski, Dortmund Menden [G] \\
\hline DL3PO [E] & EK4UC & $(-\sim 1954)$ \\
\hline DL3PP & DE6188 & Ludwig Pytlik, Zaierthofen bei Krumbach [G] \\
\hline DL3PS & DE3759 & Franz Weinwurm (-1967), Stuttgart Memmingerberg, D3KPN [G] \\
\hline DL3PX & DE1967F [E] & Bruno Zwirner (-1985), Berlin Rodenkirchen Jülich [G] \\
\hline DL3PY $[\mathrm{E}]$ & DE2633 & Peter Strauch (-1969), Köln/Nippes, D3FTI [G] \\
\hline DL3QE & EK4TE, EK4TV & $(-\sim 1956)$ \\
\hline DL3QI [CB1951] & $\mathrm{DE} 1978 \mathrm{~F}$ & $\begin{array}{l}\text { Heinz Franke, Kassel, D4SFT [CB1939], DL3QI Heinrich Franke in [CB1951] } \\
\text { not identical with DJ1SI [E] Karlheinz Franke, Frankfurt in [CB1955], [G][E] }\end{array}$ \\
\hline DL3QO & EK4CV & $(-1993)$ \\
\hline DL3QP & DE6661 & Herbert Töpmann, Pfortzheim Hannover [G] \\
\hline
\end{tabular}




\begin{tabular}{|c|c|c|}
\hline DL3QU & DE3693 & Hans Dittrich, Wilhelmshaven [G] \\
\hline DL3QX & DE2341K & Ernst Anton Hinrichs (-1983), Brake Oldenburg, D3BNK [G] \\
\hline DL3RM & D4MPR[E] & $(-1979)$ \\
\hline DL3RS & DE3720 & $\begin{array}{l}\text { Jakob Meskes, Viersen Neuss, ON8AE, PA0PK, OE8ZJK, OE8ZCL, OE1XKW, } \\
\text { HB9XCQ [G] }\end{array}$ \\
\hline DL3RU & DE3719 & Rudolf Schnurmann, Rheydt [G] \\
\hline DL3RX [E] & DE3320 & Ernst Jungermann (-1979), Duisburg Mühlheim [G] \\
\hline $\operatorname{DL} 3 \mathrm{SA}(1)[\mathrm{E}]$ & $\mathrm{DE} 2834 \mathrm{~F} / \mathrm{N}$ & Hans Zimmermann (- 1980), Langenfeld Solingen-Ohligs [G] \\
\hline $\operatorname{DL} 3 \mathrm{SA}(2)$ & DE7114 [E] & Jörg Issler, Stuttgart, D3KMN, DA3SA [G] \\
\hline DL3SI & DE6398 & Wilhelm Osterfeld, Lübbecke Wilhelmshaven [G] \\
\hline DL3SJ $[E]$ & EK4TR & $(->1994)$ \\
\hline DL3SO & DE3197 & Dipl. Ing. Rudolf Binz, Eyb, Ansbach Darmstadt, brother of DE3341 [G] \\
\hline DL3SQ [E] & DE1697N & Arthur Heck (1912-2004), Reutlingen Nürnberg, D4BJN 1933 [E], D4MCN [G] \\
\hline DL3SX & DE9033 [E] & Ottmar Popp, Helmbrechts [E] \\
\hline DL3TI & DE3710 & Heinrich Fickler (-1985), Hamburg Henstedt Norderstedt [G] \\
\hline DL3TV & DE2493D & Wilhelm Kumlehm (-1966), Holzminden [G] \\
\hline DL3UB & DE3636 & Josef Krug, Plattling [G] \\
\hline DL3UF & D4SEA & $(->1995)$ \\
\hline DL3UG & DE3695M & Rudolf Nejedlo, Greitz Bremerhaven [G] \\
\hline DL3UH & DE18890 & Heinz Engelmann, Bruchsal Oesede (-1987), D4SMO [G] \\
\hline DL3UR & DE3637 & Wilhelm Hütt, Esslingen Mettingen [G] \\
\hline DL3US & DE3063 & Willi Pfeffer, Dortmund Schwerte [G] \\
\hline DL3UY [E] & DA5AB,DA5BS & Friedrich Feldhus, Bremen-Osterholz [E] \\
\hline DL3VC & DE3684 & Heinz Brand, Rendsburg Erlangen [G] \\
\hline DL3VD [E] & EK4PU & $(-1974)$ \\
\hline DL3VE & EK4UL(1) & \\
\hline DL3VF & DE6453 & Franz-Xaver Kumpf, Magdeburg München [G] \\
\hline DL3VH & D4FVF & $(-\sim 1956)$ \\
\hline DL3VI [E] & DE3398 & Paul Hamayer (-1985), Hannover Berlin, D2HZ?, D2AV?, D3BK? [G] \\
\hline DL3VM & DE2427J & Walter Schmidt, Lübeck, DE5378, Leutnant OGF, member FWGM [G] \\
\hline DL3VR [E] & D4RWJ & $(-1978)$ \\
\hline DL3VU & DE2527K & Kurt-Heinz Lücking, Hannover Lübeck [G] \\
\hline DL3VX [E] & D4BUG & $(->1995)$ \\
\hline DL3WA & DE2862D & Dietrich Kohlgruber, Magdeburg Gummersbach [G] \\
\hline DL3WG [E] & EK4EW & $(-1991)$ \\
\hline DL3WO & DE1451G & Karl Zimmermann, Alsfeld Breslau [G] \\
\hline DL3WT [E] & DE0907F & Kurt Böhm (-1968), Berlin/Neukölln Duisburg, D4XQF [E], [G] \\
\hline DL3WV & DE3098 & Hans Martin Waitz, Darmstadt [G] \\
\hline DL3XV & DE0102K & Heinz Chrismann, Hannover [G] \\
\hline DL3XX & DE7023 & Hans Grossmann, Hannoversch-Linden [G] \\
\hline DM3NBL & DE1764 & Jürgen Haertel, Wetterberg [G] \\
\hline DL3YJ & DE3546 & Erich Pfeiffer, Münster/Gremmendorf Hannover [G] \\
\hline DL3YK(1) & DE2675U & Karl Urban Psotta (-1988), D3FEU, D3KFF [G] \\
\hline DL3YK(2) & DE3690 & Hans Kahlert, Jena Oberkochen [G] \\
\hline DL3YQ until [CB1956] & D4OHR(2) & $(-\sim 1963)$ \\
\hline DL3YU & DE6830 & Alfred Holz, Hagen Hof Lembeck [G] \\
\hline DL3ZN & EK4FV(2) & $(-\sim 1971)$ \\
\hline DL3ZZ & DE6290 [E] & $\begin{array}{l}\text { Bernhard Hinrichsmeyer, Köthen Landshut, Ausschluss aus DASD wg. Schwarzsender } \\
\text { [CQ MB 5/41] }\end{array}$ \\
\hline DL4LSM & DE3345 & Ekkehard Stolze, Leipzig [G] \\
\hline DL6AU & DE6328/DE8937 & Heinz Klein, Adendorf Twistringen, \\
\hline DL6AZ & EK4RW & $(-1963)$ \\
\hline DL6BA & DE3198 & Karl Meier (-1957), Hagen Werdohl [G] \\
\hline DL6BO & DE3665 & Otto Tüscher, Wilhelmshaven Dortmund [G] \\
\hline DL6CE & DE7018 & Heinz Günter Lehmann, Krefeld [G] \\
\hline DL6CF & DE6352 & Hermann Kientsch (-1977), Recklinghausen Lindau [G] \\
\hline DL6CG & DE6750 & Siegfried Noetz, Reutlingen Kaldenkirchen [G] \\
\hline DL6CO & DE2234H & August Poppe jr., Düsseldorf [G] \\
\hline DL6CP & DE6001 & Heinrich Rühsing (-1978), Mühlheim/Ruhr [G] \\
\hline DL6CV [E] & EK4RX & $(-\sim 1988)$ \\
\hline DL6CZ [CB1951] & DE6793 & Werner Meerkötter, Essen-Bredeney [G][E] \\
\hline DL6DC & DE6914 & Lothar Stack (-<1994), Hiddensen [G] \\
\hline DL6DD & DE2251 & Ernst Kersten (-1983), Rudersberg Bremerhaven-Lehe, D4BGJ [G] \\
\hline DL6DE [E] & DE1972?/DE60 & 4 Karl Metzger (-1982), Celle, DE6034 [G] \\
\hline DL6DF $[E]$ & DE2877T & Hermann Bender, Neustadt an der Hardt, D4OYT, DA9FE [G], started 1934 \\
\hline
\end{tabular}




\begin{tabular}{|c|c|c|}
\hline & DE3085 & Julius Weiss (-1980), Neustadt/Wese [G] \\
\hline DL6DJ & DEM2686U & Werner Voigt, Chemnitz Ludwigshafen Weindenthal Pfalz [G] \\
\hline DL6DK & DE2693T & Eugen Heinrich Klein (-1980), Haardt Neustadt, DA9RH [G] \\
\hline DL6DL & DE1979F & Karl Hussler, Berlin, D4VQT, D4VQF [KFL1944], [G] \\
\hline DL6DP [E] & DE6774 & "Jo" Hans Falz, Idar-Oberstein, DK9VQ, DA3VQ, DA3VO? [D], [G] \\
\hline DL6DS [E] & \multicolumn{2}{|c|}{ EK4UAB, EK4VF } \\
\hline DL6DX & DE6288 & Eckhard Siegel (-1983), Liegnitz Weimar Gölingen, D3KBF [G] \\
\hline DL6DZ & DE6173 & Heinz Voß, München Markt Grafing [G] \\
\hline DL6ED & DE3821 & Karl Wolf, Aschaffenburg [CB1951, CB1962][G][E] \\
\hline $\begin{array}{l}\text { DL6EE [CB51] DL8PQ [RL77] } \\
\text { DL6EF }\end{array}$ & DE6619 & Wolfgang Schnautz, Ahrensburg Neuwied [G] \\
\hline DL6EF & DE2313T & Bernhard Elbert, Worms [G] \\
\hline DL6EG & D4RGW & $(-1970)$ \\
\hline DL6EH & DE6119 & Herbert Steiger (-1983), Bad Kreuznach Haan [G] \\
\hline DL6EI & DE3265 & Jakob Unkelbach (-1964), Koblenz Oberlahnstein [G] \\
\hline DL6EN [E] & DK9UU [K] & Günter Heinzen, Bad Kreuznach [E] \\
\hline DL6EO & DE2437T [E] & Wilhelm Kneppel, Nahemühle/Nahe Bad Kreuznach Weitersborn [G] \\
\hline DL6ER & DE3391 & Hans Bender, Bad Kreuznach $[\mathrm{G}]$ \\
\hline DL6ET & DE3446 & Willi Maier (-1986), Tuttlingen, DK9WW [G] \\
\hline DL6EU & DE2801N & Paul Stucky, Schwenningen, D3DBN [G] \\
\hline DL6EV & DE3997N [E] & Johann Ameringer, Ravensburg, DA9IZ [E][G] \\
\hline DL6EW & DK9FQ & "Fritz" Walter Blersch, Ravensburg [J:14][E] \\
\hline DL6EX & DE2616P & Hermann Flad, München Tuttlingen [G] \\
\hline DL6EY $[\mathrm{E}]$ & EK4XY & $(-1989)$ \\
\hline DL6FA $[\mathrm{E}]$ & DE6731 [E] & $\begin{array}{l}\text { "Nero" Norbert Hoell (1921-1987), Tuttlingen Tübingen], DA1EA, DK9EA [E], } \\
\text { DK9AI [E], [G][E] }\end{array}$ \\
\hline DL6FD(1) & DE6452 & Wilhelm Lauffer, Mühlen/Neckar Tübingen [G] \\
\hline DL6FD(2) & DE1810F & Paul Placke (-1969), Böblingen Berlin [G] \\
\hline DL6FE & DE3913 & Karl Marquart, Tuttlingen [G] \\
\hline DL6FF $[\mathrm{E}]$ & DE6732 & $\begin{array}{l}\text { Hans Mauder (1920-2004), Erichskirch Langenargen Friedrichshafen, } \\
\text { DA1AD?, DA1WC [G][E] }\end{array}$ \\
\hline DL6FI & DE6338/DE6368 & 3 Erich Schall (-2003), Stuttgart Emmendingen, DA1EC [G] \\
\hline DL6FJ $[\mathrm{E}]$ & DE3072 & Ing. Karl Schurr (-1963), Weingarten, D3KON [G] \\
\hline DL6FO [E] & DE3874 & Willi Renziehausen, Norddeich Juest [G] \\
\hline DL6FT [E] & DE2103G & Georg Senftner (-1985), Breslau Erlangen [G] \\
\hline DL6FX [E] & SWL in 1925 & Carl Homann, Hamburg [U] \\
\hline DL6GB [E] & D4CDO & $(->1995)$ \\
\hline DL6GC $[\mathrm{E}]$ & $\mathrm{DE} 2327 \mathrm{M}[\mathrm{E}]$ & Gerhard Ladisch (-1988), Meissen Konstanz [G] \\
\hline DL6GE & DE2169N & Jakob Dörr, Insel Reichenau [G] \\
\hline DL6GG & DE2635 & Heinz Schmitz, Köln Säckingen, D3FVI [G] \\
\hline DL6GN [E] & DE2911L/H[TUC & $\begin{array}{l}\text { C] Dr. Paul Greif (-1978), Halle Bottrop Hofheim, D3FMH, D3FMB, D4FML, } \\
\text { DA3UB [G] }\end{array}$ \\
\hline DL6GP $[E]$ & DE2434B & $\begin{array}{l}\text { Erich Marquardt (-1964), Bublitz/Pommern Gr. Mackenstedt über Bremen } \\
\text { Delmenhorst, D4ZVB [E], [G] }\end{array}$ \\
\hline DL6GW & $\mathrm{DE} 0545 \mathrm{~F}[\mathrm{E}]$ & Dr. Wilhelm Schneider, Berlin [G] \\
\hline DL6HC & DE6642 & Hans Viohl, Rendsburg Kiel Eckernförde [G] \\
\hline DL6HK [CB1972] & EK4LB & $(-2012)$ \\
\hline DL6HO & DE3541 & Peter-Ernst Peters, Worms Wilhelmshaven Eckernförde [G] \\
\hline DL6HR [E] & DE9689 & Dieter Lensch, Wilhelmshaven [E] \\
\hline DL6IE & D4MOM(2) & $(-1971)$ \\
\hline DL6IR & EK4BB & $(-1980)$ \\
\hline DL6JN & DA1FA $[K]$ & Albert Ludin, Weil am Rhein [D] \\
\hline DL6JO & DE3931 & Walter Dröse, Rathenau Neukirchen Weil, DA1FB [G] \\
\hline DL6JP [E] & EK4OB & $(-1986)$ \\
\hline DL6JQ & DE6539 & Eugen Wörner (-1969), Emmendingen [G] \\
\hline DL6JT & D4MOR & $(-1972)$ \\
\hline DL6KG $[E]$ & DE6842 & Dr. Hans Georg Rath, Schwerin Belecke/Möhne Ulm [G] \\
\hline DL6KS & EK4KP & $(1910-1982)$ \\
\hline DL6KW [E] & DE9520 & Willi Seitz, Ingolstadt [E] \\
\hline DK3RI? & D4NUN(2) & $(-1967)$ \\
\hline DL6IK? & DE1285 & Heinrich Langenhan (-1997), Freiburg/Br. [G] \\
\hline DL6MF [E] & DE2728R & Richard Meyer (-1983), Wildholz [G] \\
\hline DL6MQ [E] & DE3647M [E] & William Dupke, Zittau Markelfingen [G][E] \\
\hline DL6MR & DE2483O & Julius Feininger, Karlsruhe Konstanz, D4SRO [G] \\
\hline DL6MT & DE3080 & Max Klemt (-1957), Wesermünde Bremerhaven [G] \\
\hline DL6MU & DE1687C & Günter Ullrich, Baselitz Konstanz Berlin, DE1971, D3GXO [E], [G] \\
\hline
\end{tabular}




\begin{tabular}{|c|c|c|c|}
\hline \multirow{2}{*}{\multicolumn{2}{|c|}{$\begin{array}{l}\text { DL6MZ } \\
\text { DL6NF [CB1965] }\end{array}$}} & DE3079 & Wilhelm Pelzer, Oberlahnstein [G] \\
\hline & & DE3514 & Ernst Gröger, Bassenheim [G] \\
\hline \multicolumn{2}{|c|}{ DL6NR } & DE1985H & Georg Amschler, Paderborn/Kalterherberge Münster, D4SSH [G] \\
\hline \multicolumn{2}{|l|}{ DL6OAM } & DE2692G & Wolfgang Jaeger (-1980), Breslau Holzminden Haardt Neustadt, D4HVG, DA9RH [G] \\
\hline \multicolumn{2}{|l|}{ DL6OL $[\mathrm{E}]$} & DE6118 & Erich Taxweiler, Lachendorf/Celle [G] \\
\hline \multicolumn{2}{|l|}{ DL6OS $[\mathrm{E}]$} & DE3428 & Karl Dunkelmann, Parchim Lübeck [G] \\
\hline \multicolumn{2}{|l|}{ DL6PG } & DE2442T & Eberhard Niedermeyer, Mainz/Inden [G] \\
\hline \multicolumn{2}{|c|}{ DL6PW [RL1977] } & DE6577 & Karl Romahn, Kiel Holtenau Kronshagen [G][E] \\
\hline \multicolumn{2}{|l|}{ DL6QC } & DE2576A & Otto Reinhold, Bielefeld Fischhausen, D3DVA [G] \\
\hline \multicolumn{2}{|l|}{ DL6QD } & D4DRF & $(-1960)$ \\
\hline \multicolumn{2}{|l|}{ DL6QL } & DE3216 & Hans Hoppe, Berlin Zweibrücken [G] \\
\hline \multicolumn{2}{|l|}{ DL6QM } & DE1902C & Hermann Schlosser, Haunstein Landau Pfalz [G] \\
\hline \multicolumn{2}{|l|}{ DL6QT } & DE2077V & Ernst Reinartz, Kiel Cuxhaven, D4ZTV [G] \\
\hline \multicolumn{2}{|l|}{ DL6QV } & D4LGB(2) & $(-\sim 1958)$ \\
\hline DL6QZ [E] & DL1QV & DE6409 & Lothar Meuter, Danzig Gifhorn Regensburg, YM4BD, DA4BD [G] \\
\hline \multicolumn{2}{|l|}{ DL6RE [E] } & DE2246P & Hans Müller-Schlösser, München Germering Seefeld [G] \\
\hline \multicolumn{2}{|l|}{ DL6RH $[\mathrm{E}]$} & D4ZOL & $(->1995)$ \\
\hline DL6SC & DF2TU & DE6503 & Heinrich (Heiner) Treiber (-2016), Ravensburg Stuttgart [G] \\
\hline DL6SK & [CB1953-55] & DE3998 & Erich Schmitt, Baienfurt Nürnberg $[E][G]$ \\
\hline DL6SL & & DE2799N & Albrecht Ernst (-1951), Schwenningen [G] \\
\hline DL6SS $[\mathrm{E}]$ & & $\mathrm{DE} 3284 \mathrm{~A}[\mathrm{E}]$ & Klaus Möbius, Königsberg/Pr. Oldenburg, D4CGA [G], started 1934 [QSL 1962, E] \\
\hline DL6TO & & DE6708 & Gerhard Kuster (-1978), Gosslar [G] \\
\hline DL6TQ [E] & & DE6021 & Hans Erich Böhmer, Stuttgart Aidlingen Dachtel [G] \\
\hline DL6TR $[\mathrm{E}]$ & & DE3786 & Werner Bischoff, Nussbaum bei Bretten [E] \\
\hline DL6UX & & DE2383 & Kurt Wachsmuth, Stade Hamburg [G] \\
\hline DL6VS [E] & & EK4WJ & $(->1994)$ \\
\hline DL6VU & & DE3207 & Ewald Schmidt, Solingen Halle, D3YSL [G] \\
\hline DL6WI & & DE6835 & Albrecht Helmsen (-1976), Wetzlar [G] \\
\hline DL6WJ $[\mathrm{E}]$ & DF7IL & DE6834 & Rudi Christmann, Pfortzheim Darmstadt [G] \\
\hline DL6WZ $[\mathrm{E}]$ & & DE6219 & Bruno Günther, Wilhelmshaven Lippstadt [G] \\
\hline DL6XB & & DE3517 & Georg-Hans Mutzkus (-1970), Königsberg Kaiserau [G] \\
\hline DL6XE & & D4WDH & $(-1969)$ \\
\hline DL6XJ & & DE3578 & Hans Schäff, Ansbach [G] \\
\hline DL6XK & & DE6836 & Gerhard Schliephake, Darmstadt Berlin [G] \\
\hline DL6XN [E] & & DE3823 & Helmut Bender, Aschaffenburg Elsenfeld [G] \\
\hline DL6ZA & & DE1685R & Erwin Kunert (-1987), Tumiching Nürnberg, DA2ER, DA2KW [G] \\
\hline DL6ZBS [CB199 & & DE3851T [E] & Wilfried Horn, Arheilgen Karben [G][E] \\
\hline DL6ZK & & DE6250 & Gustav Georg Thiele (Schorsch), Köln, DA6BB [G] \\
\hline DL6ZV & & DE2706F & Ernst August Koslowski (-1987), Berlin Weilheim [G] \\
\hline DL6ZZ [E] & & D4WAT & $(1910-2000)$ \\
\hline DL7AA $[E]$ & & EK4CP $[\mathrm{E}]$ & $(1911-1993)$ \\
\hline $\mathrm{DL} 7 \mathrm{AB}[\mathrm{E}]$ & & EK4IC & $(-1982)$ \\
\hline DL7AD [E] & & DE6827 & Fritz Woletz (-1989), Liegnitz Berlin, OK2RM, D4RMQ [G][E] \\
\hline DL7AE & & EK4RJ & $(-1970)$ \\
\hline DL7AG & & DE6180 & Herbert Korn (-1991), Berlin-Charlottenburg München, D4JBF, D4JHZ [G] \\
\hline DL7AH $[\mathrm{E}]$ & & DE6873 & Harry Lilienthal, Berlin München, DK7AH, DA7AH, 9X5AB, 4X2AH? [G] \\
\hline DL7AI $[\mathrm{E}]$ & & DE8503 & Peter Mack, Ahnatal [E] \\
\hline DL7AL & & EK4TA & $(-1976)$ \\
\hline DL7AM & & D4FHF & $(-\sim 1955)$ \\
\hline DL7AO $[\mathrm{E}]$ & & D5XF $[E]$ & Günter Meewes, Berlin, DA7?? [E] \\
\hline DL7AP $[\mathrm{E}]$ & & DE1549C & Fritz Peuckert (-1983), Berlin Neuenhaden, D4FIF, D3CSC, DK7AP [G] \\
\hline DL7AQ $[\mathrm{E}]$ & & EK4WG & $(->1994)$ \\
\hline $\operatorname{DL} 7 A R(1)[\mathrm{E}][\mathrm{C}$ & B49-51,62->94] & DE3716 & Dr. Karl Reichelt (-2001), Berlin Essen, founder of Berlin DARC, excluded 1952 [G] \\
\hline DL7AR(2) [CB5 & 2-59] DM2ALO & DE3166 [E] & Artur Lindholz (-1979), Berlin Stuttgart Kleinmachnow, D4ZUN, [G] \\
\hline DL7AT & & EK4AA & $(-1994)$ \\
\hline DL7AU $[\mathrm{E}]$ & & DE3060 & Gerhard Wachholz (-1983), Dankerson Minden Noren Berlin, DA7AU [G] \\
\hline DL7AV [CB51] & DL1VS [CB78] & DE6200 & $\begin{array}{l}\text { Eduard Voigt (-1978), Berlin/Karshorst, Michelbach Berlin/Niederschönhausen, } \\
\text { D4AMF, DK7BZ, DL1BX? [G] }\end{array}$ \\
\hline DL7AX & & DE3061 & $\begin{array}{l}\text { Heinrich Timmermann (-1984), Berlin Regenwalde, DA7AK [G], in } 1937 \text { expelled } \\
\text { from DASD and convicted for illegal transmission to 200RM fine/20 days prison }\end{array}$ \\
\hline DL7AY $[E]$ & & EK4BB & $(1904-1993)$ \\
\hline DL7BA & & DE3234 & Günther Kuhne, Berlin Ulm, D4YNF [G] \\
\hline DL7BB & CT1BB & DE6345 & Fritz-Karl Besgen, Aachen Berlin München, DK7BG [G] \\
\hline DL7BD & & DE1456F & Walter Ernst, Berlin, D4XTF, D4FLF [G] \\
\hline DL7BE & & DE6773 & Kurt Ernst, Berlin Braunschweig, DK9VA [G] \\
\hline
\end{tabular}




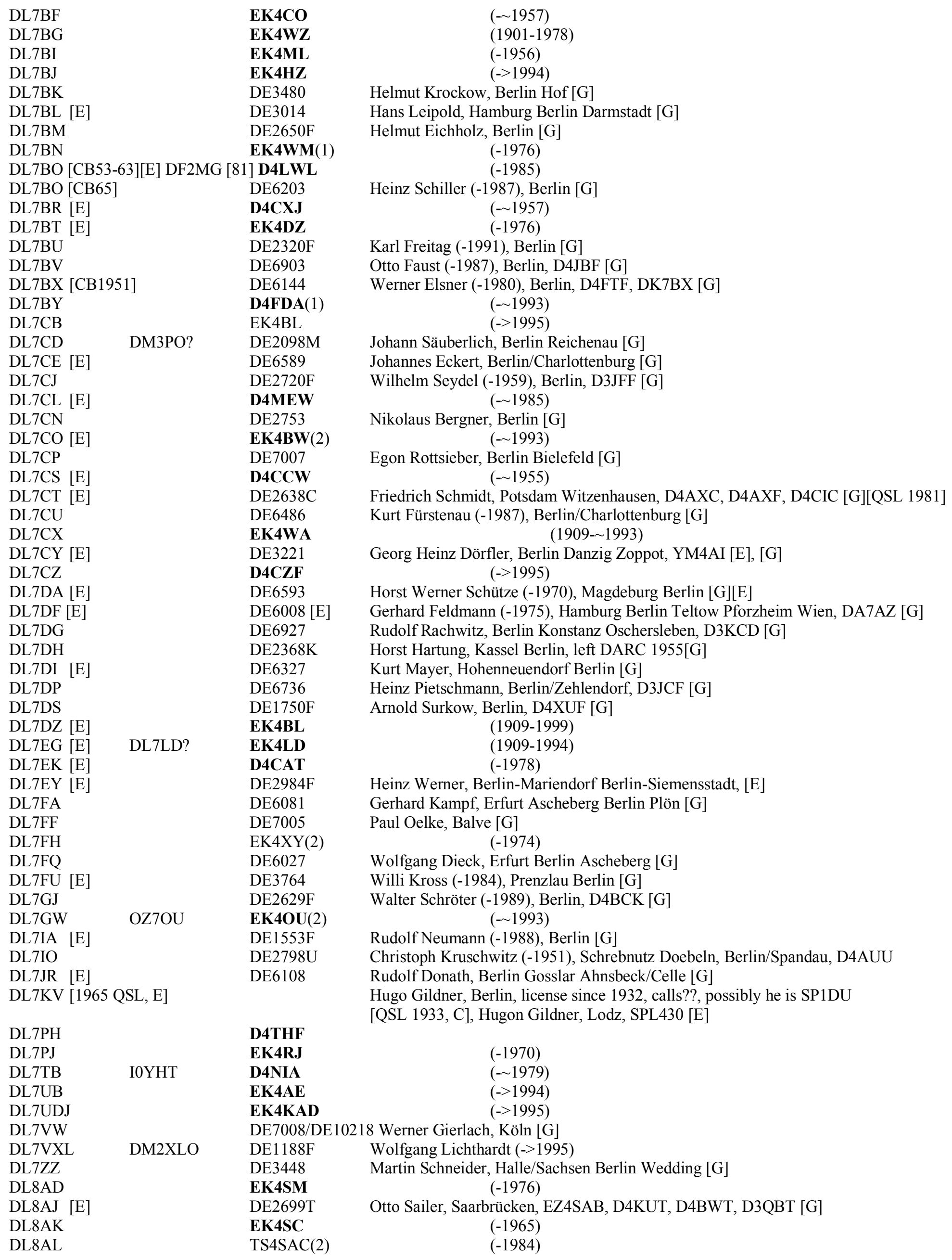


DL8AX [E]

DL8AZ

DL8CI

DL8IF

DL8OB [E]

DL8PQ [RL77] DL6EE [CB51]

DL8QA (-1971) DM2FSO

DL9AB

DL9AI

DL9AZ [E]

DL9BH [E]

DL9BO

DL9DF

DL9DQ [E]

DL9DW [E]

DL9EJ

DL9FI [E]

DL9FY

DL9HH

DL9HM [E]

DL9HV

DL9HX [E]

DL9IF

DL9IH

DL9JU

DL9JY

DL9MR

DL9NW

DL9PN

DL9PV

DL9QQ

DL9RZ

DL9SE [E]

DL9UH

DL9UJ

DL9US

DL9VQ

DL9WY[E]

DL9XR

DL9YB

DL9YF

DL9YM

DL9ZG
EK4SR(1)

EK4SZ

DE3783

DE3977

EK4MW

DE6619

DE1894C

DE6277

DE3955

DE2913

DE6040

DE3896

DE3423

DE7348

DE6550

DE2819H

D4VNB

DE3935

DE2431P

EK40W

D4XRT

EK4UA(1)

DE2168N

DE7083

DE7026

D4LRX

DE3723

D4CIC

DE3086

DE1176

DE3944

EK4GN

DE9627

DE6381

DE3577R [E]

DE2497K [E]

DE3621

DE2136G

DE3938

DE3233

DE1953H

DE2085H

EK4ABP

DM2ABB [E]

DM2ABC [E] DM2ACA DL1OV

$\mathrm{DM} 2 \mathrm{ABH}$

DM2ABK [E]

DM2ABM

Y21BM

DM2ABN [E]

DM2ADH

DM2ACA [E] DM2ABC DL1OV

$\mathrm{DM} 2 \mathrm{ACB}[\mathrm{E}]$

DM2ACE

DM2ACM [E] DL1LWM

DM2ACN [E][CB1959]

DM2ACO [CB1962-78]

DM2ADB [E]

DM2ADD

DM2FGO [E]

DM2ADE [E]

DM2ADM Y21DM

DM2ADO [E] Y21DO, DL7UDJ

DM2AEB [E]

DM2AEJ [E]
EK4AL

EK4EY

EK4KAD

DE3040

D4GOS

DE5331H

DE1566L/F

EK4EY

DE6999

DE1755G

EK4MX

D4CDM

D4CDM

DE2549F

DE2503U

EK4KAD

DE1907U

EK4KAD

D4BUC(2)

EK4BH
$(-1965)$

(-1959)

Hans Kreplien, Berlin Langwitz [G]

Ernst Apel, Braunschweig [G]

$(-1990)$

Wolfgang Schnautz, Ahrensburg Neuwied [G]

Günther Mangelsdorff (-1979), Potsdam Bad Homburg, D4ACR, D4FSC from Saloniki

during German occup., DK9FA [G], host of Gus Browning [Browning, 1965]

Wolfram Quast, Stettin Hasenlünne, Tucson/AZ, WB7WSI [G]

Walter Tautz, Norddeich Düsseldorf [G]

Hagen Zernin (-1980), Hamburg Langenhagen [G]

Karl Fischinger, Nürtingen Neckartenzlingen [G]

Karl Noller, Heilbronn [G]

Walter Pfister (-1987), Freiburg, D4MGO [G]

Helmut K. Seike (-2012), Fürth Surprise AZ, AA8GQ [E]

Erich Groth, Freiburg (ausgeschieden 1954 but rejoined)

Adolf Bär, Wuppertal Barmen Aurich Sandhorst [G] $(-\sim 1967)$

Hermann Brünning, Bocholt [G]

Franz-Xaver Wagner, Landshut [G]

$(-1971)$

$(-\sim 1971)$

$(-1968)$

Erich Götz (-1974), Stuttgart Tübingen Rottenburg, DA1EG [G]

Gerhard Käsemann (-2004), Gotha Lindau [G]

Ludwig Wagner, München [G]

$$
(-\sim 1990)
$$

Wolfgang Bradinal, Osnabrück, DK9JN [G]

$$
\text { (- 1993) }
$$

Ing Karl Körber, Böblingen Ingolstadt [G]

Günther Seide, Ingolstadt Bad Tölz [G]

Joachim Schulze, Sarstedt [G]

$$
(-\sim 1993)
$$

Fritz Ilgner, Bielefeld [E]

Dr. Norbert Bühler, Stuttgart [G]

Willy Grimm (-1975), Nürnberg Demmin Ulm [G]

Volkmar Vincentz, Hannover Ulm Neuhaus [G]

Rudolf Friedrich, Darmstadt Wallhausen Krs. Kreuznach [G]

Paul Wolny (-1972), Breslau Aachen, D3HCG [G]

Willy Bock, Bochum-Riemke [G]

Hans Bebert, Berlin Düren [G]

Theodor Nakath (-1969), Elberfeld Düsseldorf [G]

Alfred Keil, Duisburg [G]

$(->1994)$

$$
\begin{aligned}
& (-\sim 1963) \\
& (-1984) \\
& (->1995)
\end{aligned}
$$

Karl Rothammel (1914-1987), Fürth München Sonneberg, Y21BK [G]

$$
(-\sim 1985)
$$

Kurt Georg Bräuer (-1977), Karl Marx Stadt, [G][CB1954]

Franz Otto Baer, Halle/Sa. Tempelhof, Op: D4AEX, AEX [CB1954] $(-1984)$

Heinz-Gustav Stiehm, Schwerin, DM5MM/MM, ZA2ACB, Y21CB [G]

Herbert Richter, Liegnitz, D4KMG, [CB1959][G]

$$
\begin{aligned}
& (->1995) \\
& (-\sim 1980) \\
& (-\sim 1980)
\end{aligned}
$$

Otto Buchelt, Berlin Grabow/Meckl., D4XJF, [CB1959][G]

Werner Krebs (- 1987), Leipzig Berlin, D3FGU, Y26GO [G] $(->1995)$

Wolfgang Nabroth, Leipzig, D3FIU [G][CB1954]

$(->1995)$

$(-\sim 1980)$

$(-1978)$ 
DM2AEM

DM2AEN [E] [CB1958]

DM2AEO

DM2AHM [E] Y21KM

DM2AHN

DM2AIN

DM2AIO

DM2ALO DL7AR

DM2AND [E] D4FBC

DM2ANG

DM2ANM [CB1956]

DM2DZL Y24ZL DL2DZL

DM2FGO [E] Y26GO [CB88]

DM2FHH

DM2FSO DL8QA DK9FA

DM2OOO [E][CB1958]

DM2XLO [E] Y25LO, DL7VXL DE1188F

DM3MO Y24MO D4FPF

DM3NBL [E] DL3YAI DE1764

DM3WJL DM2DZL

DM3WMI

DM3WTM

$\begin{array}{lcl}\text { CT1BB [E] } & \text { DL7BB } & \text { DE6345 } \\ \text { EI2TQ } & \text { DF3XX } & \text { EK4TV(1) } \\ \text { F4SM } & & \text { EK4SM(1) } \\ \text { HB9ACS } & \text { DL1MB OE5OW } & \text { DE6825 } \\ \text { HB9AWV } & \text { DJ4WN } & \text { EK4AS } \\ \text { HB9SI } & \text { PA0BB } & \text { D4FLA } \\ \text { I1UNO } & & \text { EK4GC } \\ \text { I0YHT } & \text { DL7TB } & \text { D4NIA } \\ \text { K6TE } & & \text { EK4LAJ } \\ \text { KA8OSS } & \text { DL1YN } & \text { DE3022 [E] } \\ \text { LU3DVF } & \text { DL1YN } & \text { DE3022 [E] } \\ \text { LU7DH } & & \text { EK4JB } \\ \text { OA4FN } & & \text { DE1970F }\end{array}$

OE1AW

OE1CM

OE1EJ.

OE1ES

OE1FH

OE1FP

OE1IU

OE1KN

OE1SJ

OE1WR

OE2HW

OE2JP

OE2PJL

OE2WC

OE3CLM

OE3FL

OE3JZ

OE3RP

OE5BJ

OE5JB

OE5LE

OE6DK

OE6KCG

OE6KW

DE2677U

DE6947U

DE6482

DE3166 [E]

DE3983 [C]

D4BZH

D4FRG(1)

DE2503U

DE3911

DE1894C

DE2324

DE1764

DE6783

DE6390

EK4OO

DE6537

DE6471

DE6478

DE6511

DE7029

DE6573

DE6475

DE6472

DE7081

DE6581

EK4DW

DE6583

DE6645
DE1868U Herbert Trepte, Leipzig Dresden [G][CB1954], made on June 15, 1953 first contact

from the GDR with a station in Berlin [Berlin-Chronik, 2015]

D4GJM, D4NFKHans R. Sommer (-1981) [G][E][DM6WAN]

DE1779F Willi (Friedrich) Fussenegger (-1988), Berlin [G]

EK4MG (- 1994)

Kurt Heinze, Zwickau, D4QYU [G]

Walter Kosche, Leipzig Chemnitz [G][TUC]

Franz Bohn, Berlin [G]

Artur Lindholz (-1979)

$(-2004)$

$(-1975)$

G. Reidemeister, Magdeburg [G]

$(-\sim 1957)$

(1910-2008)

Werner Krebs (- 1987), Leipzig Berlin, D3FGU, DM2ADD [G]

Rudi Böckle, Ballenstedt Hannover, Y26HH [G]

DL8QA, Günther Mangelsdorff

Horst Baier, Dresden Berlin-Hohenschönhausen, started 1926 [G]

Wolfgang Lichthardt (->1995), Berlin Kthen, D4XLD [KFL1944], [G][C],

host of Gus Browning [Browning, 1965]

(- 1993)

Jürgen Haertel, Wetterberg [G]

Heinz Liebenroth, Nordhausen [G]

Strutz, Berlin [G]

Fritz-Karl Besgen, Aachen Berlin München, DK7BG [G]

$(-\sim 1990)$

$(-1976)$

Karl Weinberger, Ulm Steir Zimikon [G]

(1911-1988)

(1901- 1970)

$(-1985)$

$(-\sim 1979)$

$(1915-1999)$

[CB1993]

[CB1962]

$(-1983)$

Felix Cremers (-1968), Berlin/Lankwitz Karlsruhe Lima, D4XVF [KFL1944], OA4FN [CB1965], CE3TE?, DL3NW [CB1951-1953], [G], operated in the 1920ies from F8AZ, German delegate to the IARU founding meeting in Paris, 1925 [K]

DE6646 Karl Warnick, Wien [G]

DE1086/DE6509/DE7170 Dr. Erich Lippert, Mitbenutzer Olga Lippert, Wien, D4AZS, OE212 [G]

D4JNW (-1966)

DE6505 Ing. Emmanuel Strunz, Wien, OE083 [G]

DE1103/DE6507 Fritz/Richard Haas (-1981), Salzburg Wien, EAJI, UO1FH [E], [G]

DE6468 Franz Polacek, OE017, Wien [G]

DE7024 Karl Nemecek (-1978), Wien, OE301, D3JLD

DE6512 Josef Safka, Wien, OE093, D3JIS [G]

Wilhelm Kallischek, Teltow Wien, D4UZC, D4UZS [G]

Hans Wieder, UO1WG, Salzburg

Josef Potzinger, Salzburg, OE251 [G]

Hans Pichler, Wien, OE219 [G]

Walter Craigler, Eggenburg Salzburg, OE327 [G]

Carl Lessainsky, Wollersdorf, OE241 [G]

Franz Lehner, Linz/Donau, UO3F, OE081 [G]

Ing. Johann Zadra, Salzburg, D4IFS, DA1BB? [G]

Richard Payer (-1982), Knittelfeld Steinach, OE196 [G]

Hans Breuer, Hohenems St. Marienkirchen, OE198 [G]

DE1579/DE7030 Josef Bieberauer, Weibern Haag, OE057 [G]

DE7020 Ludwig Ellinger (-1951), Kremsmünster Wien, OE714, D3JMS [G]
Ing. Erich Kiennert, Loeben, D3JWW [G]

Karl Went, Graz Reutte, OE106 [G] 


\begin{tabular}{|c|c|c|c|}
\hline \multicolumn{2}{|l|}{ OE6KZ } & DE6582 & Karl Kurz, Wies Deutschlandberg, OE040 [G] \\
\hline \multicolumn{2}{|l|}{ OE6TH } & DE6584 & Emmerich Harmet, Graz, OE195 [G] \\
\hline \multicolumn{2}{|l|}{ OE6TJ } & DE6716 & Josef Tockner, Trofaiach, OE199 [G] \\
\hline \multicolumn{2}{|l|}{ OE7FW } & DE6647 & Friedrich Waitz, OE181, D4ULW [G] \\
\hline \multicolumn{2}{|l|}{ OE7PK } & DE6644 & Herbert Machyttka, Insbruck, DJ1OMA?, OE176 [G] \\
\hline OE8FG & DJ1HX & DE6672 & Ing. Felix Gaggi, Klagenfurt [G] \\
\hline $\mathrm{OZ2CL}$ & $3 \mathrm{~A} 2 \mathrm{CL}$ & EK4CL, EK4AF & $(1906-1984)$ \\
\hline \multicolumn{2}{|l|}{ PA0PR } & D4CFH & $(-1978)$ \\
\hline \multicolumn{2}{|l|}{ PA0RU } & D4CFH & $(-1978)$ \\
\hline TI2IO [E] & DL3IO & DE6720 & Hans Schulze-Allen, Soest San Jose Costa Rica [G] \\
\hline \multicolumn{2}{|l|}{$\mathrm{XE} 1 \mathrm{CD}$} & EK4TH & $(-\sim 1993)$ \\
\hline \multicolumn{2}{|l|}{ W2BSR [E] } & D4KAN & \\
\hline W2TO & DL1XX W2HIU & DE6347 & Hans Meurer (-2007), Detmold Ridgewood/NJ, president NJDXA [G] \\
\hline VK2AOU [E] & DL1EZ(1) & DE3562 [E] & Hans Rückert, Selb Suhl Wismar Berlin Sydney, DA2W? [H] \\
\hline VK2PK & VK2APK & DE6510 & Dietmar Kiesewetter, Krematen Krems Dullwick Hill [G] \\
\hline YA1AO [E] & & EK4GC & \\
\hline $\mathrm{ZS} 2 \mathrm{CM}$ & & EK4QY & $(-\sim 1952)$ \\
\hline ZS3YK $[E]$ & DL3YK & DE2675U & DL3YK, Karl Urban Psotta (-1988) \\
\hline ZS6BKJ & DL1OG & D4BWL & $(-1979)$ \\
\hline 4X4BX [E] & & YL2BA & $(-1986)$ \\
\hline
\end{tabular}




\begin{tabular}{|c|c|c|}
\hline D4KLX [E] & "Costa" & München ?? \\
\hline DA1AY [E] & "Will" & Stuttgart ?? \\
\hline $\mathrm{DA} 1 \mathrm{BG}[\mathrm{C}]$ & "Joe" & near Karlsruhe ?? \\
\hline $\mathrm{DA} 2 \mathrm{BF}[\mathrm{E}]$ & "Joe" & Bavarian Alps ?? \\
\hline DA1HX $[\mathrm{K}]$ ex DA1CE & ?? & ?? \\
\hline DA2CA [E] & "Hans" & München ?? \\
\hline $\mathrm{DA} 3 \mathrm{GA}[\mathrm{K}]$ & ?? & ?? \\
\hline DA4DE & $? ?$ & $? ?$ \\
\hline DA4NC [E] & "Peter" & near Köln ?? \\
\hline DA4XA & DE2916 & Peter Mosel, Köln [G] \\
\hline DA4ZH & DE2719F & Rolf Hübner, Berlin Essen Moers [G] \\
\hline DA5AM [J:14] & $? ?$ & Herbert ?? \\
\hline DA5BB & ?? & Günther ?? [J:14] \\
\hline DA5BH & DE5432 & Rudi Schulz [G] \\
\hline DA5BL, DA5IL [E] & “Ted" & Braunschweig (different from DL1QU?) ?? \\
\hline $\mathrm{DA} 5 \mathrm{FW}[\mathrm{K}]$ & $? ?$ & ?? [J:14] \\
\hline DA5HF & ?? & ??, Bremen [J:14] \\
\hline DA5LL & "Harry" & Harry Müller, ?? [J:14] \\
\hline DA5LY & $? ?$ & Karl-Heinz ?? [J:14] \\
\hline DA5MF & $? ?$ & $? ?$ \\
\hline DA5SB [J:14] & $? ?$ & $? ?$ \\
\hline DA5XH [J:14] & $? ?$ & ?? \\
\hline DA5XK & $? ?$ & ??, Bremerhaven [J:14] \\
\hline DA5XW & $? ?$ & Reinhold ??, X Stadt [J:14] \\
\hline DA5XZ [J:14] & ?? & ?? \\
\hline $\mathrm{DA} 5 \mathrm{ZZ}[\mathrm{K}]$ & "Wim" & Göttingen, Alter ham im neuen Kleide ?? \\
\hline DA6BH & DE2425J & Hans Buchwald, Hamburg-Billstedt [G] \\
\hline DA6FD [E] & "Walther" & Burg/Schleswig-Holstein ?? \\
\hline $\mathrm{DA} 6 \mathrm{FU}[\mathrm{C}]$ & "Rupo" & $? ?$ \\
\hline DA6LI [E] & "Willi" & Hamburg [E] \\
\hline $\mathrm{DA} 7 \mathrm{AC}[\mathrm{E}][\mathrm{K}]$ & "Horst" & near Berlin, D4XXC, D6AC?? \\
\hline DA7CQ, DA1HB $[\mathrm{E}][\mathrm{K}]$ & "Hans" & Berlin, near Heilbronn ?? \\
\hline DA7LX & DE6847 & Karlheinz Muller, Berlin Wiesbaden, DL1CM? [G][E] \\
\hline DA7RB [E] & "Hans" & near Berlin ?? \\
\hline DA7RF & DE3232 & Willi Olczewski, Berlin, D3JEF [G] \\
\hline DA7ZA [E] & "Fredy" & Berlin ?? \\
\hline DA8RM [E] & "Kurt" & near Berlin, DK8RM ?? \\
\hline DA8SL [E] & "Max" & Saxony, DK8SL ?? \\
\hline DA0RK [E] & “Bill” & Roland Kummer, Triptis/Thüringen (DA0 not organized by SAC) \\
\hline
\end{tabular}


(5) References:

[Berlin-Chronik, 2015]

[Bernhard]

[Bocholt City Info]

[Browning, 1965]

[Bürkle, 1950]

[Bürkle de la Camp, 1969] [C]

[Campbell, 2019]

[CB1951]

[CIA report 1951]

[Corsepius 1891]

[Corsepius 2013]

[CQ-MB 6/1927]

[D]

[DARC Hessen, 1997]

[DASD, 1931]

[DeSoto, 1936]

[DIG Members]

[DJ3JD]

[DJ4ZC]

[DK5GA letter]

[DL2SWR]

[DL7AY letter]

[DM6WAN]

[E]

[ES3GZ]

[F:10]

[Forum Jacob Pins]

[G]

[Grämer, 1953]

[Günther, 1925]

[Günther Culatti, 1925]

[Haring, 2006]

[Harranth, 1996]

[Hausdorff, 1925]

[Hess, 1946, 1947]

[Hess \& Rawer, 1960]

[Hilton, 1999]

[HH]
Chronik des Distriktes Berlin im Deutschen Amateur Radio Club e.V. 1947-2015,

https://dl0bn.de/chronik/Berlin-Chronik.pdf

Bernhard/Bernhardine navigation system used by the Luftwaffe based on Hellschreiber (Dr. Ing. Rudolf

Hell, Berlin Dahlem, D2BW [RL-D2 1935])

https://www.nonstopsystems.com/radio/hellschreiber-modes-other-hell.htm

https://www.bocholt.de/rathaus/kultur-und-bildung/erinnerungskultur/erinnerungsorte/beuker/

Gus Browning: Gus: Part V. 73 Magazine, 9/1965, p. 66-68

Helmut Bürkle: Handbuch für den Kurzwellenamateur. Waidmannsche Verlagsbuchhandlung Berlin, 1950

Heinrich Bürkle de la Camp: Chirurgenverzeichnis. Springer (5th edition), 1969

QSL online collection K8CX

http://hamgallery.com/qsl/

Bruce B. Campbell: The radio hobby, private associations, and the challenge of modernity in Germany.

Palgrave Studies in the History of Science and Technology, 2019

Callbook, international listings, 1951, etc. invaluable scans by archivist Stephen J Lampereur KB9MWR!

CIA report on research establishments in the US-zone of Germany 1946-1948

Max Corsepius: Theoretische und praktische Untersuchungen zur Konstruktion magnetischer Maschinen, Springer Berlin 1891

Yolanda Corsepius: Max Corsepius in the Terceira Island Prison Camp.

https:/portugal1914.org/portal/pt/memorias/historias/item/468-max-corsepius-no-campo-de-prisioneiros-da-ilha-terceira

CQ-Mitteilungsblatt, monthly magazine of the Deutscher Amateur Sendedienst (DASD), June 1927, etc.,

http://dokufunk.org/

50 Jahre Amateurfunk in Hessen 1947-1997

https://d10bn.de/archiv/distrikte/Chronik\%20F.pdf

DASD: Kurzwellentechnik, Ein Leitfaden für den Amateur. Verlag Rothgiesser und Diesing AG, Berlin 1931

Clinton B. DeSoto: 200 meters and down. The American Radio League, 1936

Diplom Interessensgemeinschaft, member list

https://diplom-interessen-gruppe.info/

Dr. Eckard Viel: Funk in Braunschweig, http://www.dj3jd.eu

Personal communication with K6EE about Dr. Curt Lamm, EK4CL, 2008

Letter from Rudolf Horkheimer, DK5GA, to Otto Tuescher, DL6BO, 1970, K6EE collection

Biography of Kurt Heinrich, Akademie Wismar

http://www.dl2swr.afu-wismar.de/heinrich.html

Letter from Günther Noack to DC9TK, 1971

Mitglieder Ortsgruppe Chemnitz, https://dm6wan.de/ortsgruppe-dasd.html

QSL collection K6EE

Slides on history of amateur radio in Estonia

https://www.erau.ee/images/ERAU_ajalugu/es history_veebile_eng.pdf

Ernst Fendler, Günther Noack: Geschichte des Amateurfunks im Wandel der Zeit. DARC Verlag, 1986; page 10, etc.

Geflohene Schüler des KWG Höxter: $\quad$ http://www.jacob-pins.de/?article id=500\&clang=0

List of DE listener identifier numbers, put together after WW2 by Ludwig Grünberger, DL6KQ

https://www.darc.de/der-club/distrikte/e/distriktarchiv/\#c244082

Carl Grämer: Bauanleitung: 6-Kreis-Allstrom-Superhet. Deutsche Funktechnik Zeitschrift Radio

Fernsehen Akustik DDR, Heft 6 Röhren, 6/1953

Hanns Günther, Ed., Authors: Fr. Denker, Dr. P. Lertes, Dr. Franz Fuchs, Dr. E. Nesper.

Wie erwerbe ich die Versuchserlaubnis. Frank'sche Verlagsbuchhandlung, Stuttgart, 1925

Hanns Günther, C. Culatti: Wer gibt? Die Funkstationen der Welt. Frank'sche Verlagsbuchhandlung

Stuttgart, 1925

https://www.viehl-radio.de/homeda/chronik/wergibt.pdf

Kristen Haring: Ham Radio's technical culture. MIT Press 2006

Wolf Harranth: Aus den Schätzen der QSL-collection. Theuberger Verlag, 1996

Max Hausdorff: Radio-Leitfaden; praktisches Handbuch für den Radiohandel, behandelt von Dr. Ing.

Max Hausdorff. Union Deutsche Verlagsgesellschaft, 1925

Hanns Albrecht Hess: Untersuchungen an Kurzwellen-Echosignalen. Zeitschrift für

Naturforschung A 1.9 (1946): 499-505.

Hanns Albrecht Hess: Untersuchungen an Kurzwellen-Echosignalen II. Teil. Zeitschrift für

Naturforschung A 2.9 (1947): 528-535.

Hess, Hanns A., and K. Rawer. Research on and recording of RF-signals from satellites. AIR

FORCE CAMBRIDGE RESEARCH LABS LG HANSCOM FIELD MASS, 1960.

Stanley E. Hilton: Hitler's Secret War In South America, 1939-1945: German Military Espionage

and Allied Counterespionage in Brazil. Lousiana State University Press, 1999

Lists of radio amateurs in Hamburg and Northern Germany, DJ1GE 
[HSV Database]

[Jasenovac]

$[\mathrm{J}: 8]$

[Jürgens, 1997]

[K]

[KFL1944]

[Kofes, 1938]

[Kotowski \& Wisbar, 1941]

[Krotkofalewski Polski 12/1931]

[Lecorte, 2015]

[Lertes, 1926]

[Lickfeld, 1985]

[Lorenz AG Festschrift, 1955]

[LY2YR]

[Kienzle, 2008]

[M 1927]

[McGaha, 2009]

[MFS, 2008]

[Modelleisenbahn 8/1971]

[Müller, 2017]

[Müller \& Scheuering, 1944]

[OOTC 2818]

$[\mathrm{OP}]$

[OZ7DX]

[P 1927]

[Patent, 1937]

[Puschmann, 1962]

[Puschmann \& Körner, 1969]

[RL1985]

[RL-D2 1935]

[S]

[Schmitt-Enboom \& Stoll, 2015]

[Schwarz, 2015]

[Schick 2016]

[Simon \& Goldsmith, 1913] https://www.darc.de/der-club/distrikte/e/distriktarchiv/\#c244082

Holocaust survivors and victims database

https://www.ushmm.org/

Jasenovac Memorial Site

https://www.jusp-jasenovac.hr/

Leo H. Jung (-2004), DH4IAB, QSLs erzählen deutsche Amateurfunkgeschichte. Folge 8,

Funk-Telegramm 11/2003. Dokufunk.org, etc.

Franz J. Jürgens: Wir waren ja eigentlich Deutsche. Aufbau Tb 1997 Berlin, ISBN 9783746613383

Wolfram Körner: Die Geschichte des Amateurfunks in Deutschland bis 1963. Körner Verlag, 1963 - on archive.org scanned by HB9THJ

http://www.hb9thj.ch/P01/Archiv/Books/Geschichte-des-Amateurfunks-Koerner-DL1CU-1963.pdf

German war licenses list of 1944

Albert Kofes: Ausbreitung elektromagnetischer Wellen. Lorenz AG, 1938

Paul Kotowski, Herward Wisbar: Drahtloser Überseeverkehr. Reihe Physik und Technik der Gegenwart

Telefunken Laboratorium Berlin, Hirtzel, Leipzig 1941

Magazine of the Polish Amateur Radio Club 12/1931 etc.

Thomas Lecorte: Gauner, Nazis und Agenten - Stay Behind in der Bundesrepublik 1948-1960.

http://www.lecorte.de/wp/wp-content/uploads/2015/06/Staybehind 2015.pdf

P. Lertes: Die Telephonie-Sender. Julius Springer, 1926

Karl Gerhard Lickfeld, DL3FM: Ein Pionier des Amateurfunks in Deutschland. Selbstverlag 1985

- Ders. Prof. Dr. Wilhelm Ludolf Schmitz in: cq-DL 2/1989 page 103 and following

75 Jahre Lorenz (1880-1955), Festschrift zum Firmenjubiläum, 1955

https://www.cdvandt.org/75-jahre-lorenz.htm

History of amateur radio in Lithuania

https://ly2yr.wordpress.com/ham-radio-history/lietuvos-ham-radijo-istorija/

Paula Kienzle: Spuren sichern für alle Generationen: Die Juden in Rottenburg im 19ten und 20igsten

Jahrhundert. Lit Verlag, 2008, ISBN-10: 3825811565, ISBN-13: 978-3825811563

Dipl.-Ing. Rudolf Mertz: Die privaten Funksendeanlagen in Deutschland. Der Deutsche Rundfunk $1 / 1927$

Richard L. McGaha: The Politics of Espionage: Nazi Diplomats and Spies in Argentina, 1933-1945.

Dissertation, College of Art and Sciences, Ohio University, 2009

Anatomie der Staatssicherheit, Geschichte, Struktur und Methoden - MfS Handbuch. 2008

https:/www.bstu.de/assets/bstu/de/Publikationen/HB bstu HV-A.pdf

Interview with Rudolf Insam, EK4UD. Die Modelleisenbahn, issue 8/1971 page 85. For excerpts:

https:/trixstadt.de/trix-express-geschichte/1941-kriegsbedingtes-ende-der-spielzeugproduktion/

Armin Müller: Wellenkrieg: Agentenfunk und Funkaufklärung des Bundesnachrichtendienstes 1945-

1968. Links Verlag, 2017

Günther Müller, Fritz Scheuering: Sprung über Kreta, ein Bild- und Kampfbericht. Oldenburg:

Gerhard Stalling Verlag, 1944

OOTC member listing, member number 2818, etc

https://ootc.us/

List of radio amateurs in East Prussia, courtesy of DJ1GE collection

Personal communication with K6EE about Dr. Curt Lamm, EK4CL, 2008

Hans W. Priwin, DE0783: Kurzwellen-Verkehr Ein Handbuch fuer den Amateur nach dem Stande vom 1. Januar 1929, Rothgiesser \& Giesing AG, Berlin N24, 1928

Karl Mueller, Dipl-Ing Hubert Seeanner: Device for securing dental nerve needles against falling in the mouth. German patent DE672565C, 1937

https://patents.google.com/patent/DE672565C

Bernhard Puschmann: Der Weg zum Amateurfunk. Franck'sche Verlagshandlung, 1962, and later editions

Bernhard Puschmann, Wolfram Körner: Was ist Amateur-Radio? Hier erfahren sie alles genau. Körner

Verlag, 1969

Rufzeichenliste der Deutschen Amateurfunkstellen 1985, etc

Rufzeichenliste der von der Deutschen Reichspost genehmigten privaten Versuchsanlagen mit

Fernstrahlung -D2xx Rufzeichen, 1935

https://www.viehl-radio.de/homeda/chronik/d2liste35.pdf

QSL online collection SP9EWM

http://sp9ewm.webd.pl/karty/index.php

Erich Schmitt-Eenboom, Ulrich Stoll: Die Partisanen der NATO. Stay-Behind-Organisationen in Deutschland 1946-1991. Ch. Links, Berlin 2015, ISBN 978-3-86153-840-0

Jürgen Schwarz: Direktoren und Rektoren der Hochschule Anhalt und ihrer Vorgängereinrichtungen $2^{\text {nd }}$ Edition 2015, ISBN 978-3-86011-066-9

Rolf Schick: Amateurfunk in Esslingen in der Zeit nach dem 2-ten Weltkrieg: Erinnerungen an die Jahre 1946-1965, 2016

EJ Simon, AN Goldsmith: The Seibt direct indicating wave meter. Proc. I. R. E, Vol. 1-2:3-22, 1913 
[Solomon, 2011]

[Sommer et al., 2022]

[Spiegel 6/7/1950]

[Spiegel 51/1956]

[Staritz, 1985]

[Teuchert, 1953]

[Torbarina]

[TUC]

[U]

[V]

[Van der Mey, 2012]

[VDE Listing, 1925]

[VFDB, 2000]

[Vizethum, 1985]

[W]

[West, 2019]

[Wiegand, 1908]

[Zschaler 2000]

[4X4KK]
A. Solomon: The FOX Film Corporation 1915-1935. McFarland \& Company Publishers, 2011

Sommer, Friedrich T., Steffen Hamperl, and Gerd Hoyer. 2022. "Who Was Who in the Aether of the Weimar Republic? Tracing the Callbooks That Never Existed." MediArXiv. January 25.

doi:10.33767/osf.io/rz64t

Article about the introduction of TV in Germany and the struggles between different experts on what norms to adopt. Two familiar names appear in this article, Karl Kleefeld, EK4TD and Werner Nestel, EK4LV: Fernsehen: Pistole auf die Brust.

https://www.spiegel.de/spiegel/print/d-44448872.html

At a Munich high-society party, Fritz Deckel, only son of Dr. Hans C. Deckel, pushes a well-known finance broker' wife in her fur coat in the swimming pool, then jumps himself. Legal action was announced by the finance broker.

https://www.spiegel.de/spiegel/print/d-43064865.html

Rudolf F. Staritz, DL3CS: Abwehrfunk - Funkabwehr Technik und Verfahren der Spionagefunkdienste. 1985. https://www.cryptomuseum.com/people/staritz/rudolf.htm

Ing. Hans Teuchert: Grundlagen der Elektrotechnik, Fachbuchverlag GMBH Leipzig, 1953

Mladen Torbarina, EJ7XX: History of amateur radio in Yugoslavia

https://www.croatianhistory.net/gif/science/tkalcevic/mladen torbarina.pdf

TU Chemnitz QSL Archive, DM6WAN

SM6UA Fotosammlung deutscher Amateurfunkstationen 1924-1931, DJ1GE collection

https://www.darc.de/der-club/distrikte/e/distriktarchiv/\#c244082

QSL online collection F2VX

http://lesnouvellesdx.fr/

Michiel Van der Mey: Dornier Wal: A light coming over the sea. Perfect Paperback, 2012

Verband Deutscher Elektrotechniker Verzeichnis 1925

Festschrift 50 Jahre VFDB, page 77 (OV Z22), 2000

https://www.vfdb.org/files/festschriften/Festschrift50 5.pdf

Hans Vizethum, DL1BZ: Letter to Rudolf F. Staritz 1985 [E]

https://kriegstagebuch.edith-kohlbach.de/eiserne-hand.html

Wikipedia

Nigel West: Codeword Overlord: Axis espionage and the D-day landings. The History Press, 2019

Karl H. von Wiegand: “Stop it kid!” cries congress to the american boy. San Francisco Call, March 29, 1908

http://earlyradiohistory.us/1908stp.htm

Hans Zschaler: TRIX-Vereinigte Spielwarenfabriken Teil 1. On page 48 a photo of Rudolf Insam, EK4UD with Hermann Goering who was presented with a TRIX model train for his 50th birthday. https://www.qcwa.org/4x4bx-14374-sk.htm 